Samples were disaggregated with an electric mixer, washed to remove clay, and then centrifuged in tetrabromoethane (2.95 grams per $\mathrm{cm} 3)$ to separate dense minerals. Euhedral apatite crystals that were determined to be free of inclusions by reflected light microscopy were selected for analysis. All samples were analyzed using wavelength dispersive spectrometers on a JEOL JXA 8600 Superprobe housed in the Department of Earth Sciences at Syracuse University. Using Geller Analytical's dSpec software, Mg, $\mathrm{Mn}, \mathrm{Fe}, \mathrm{Y}$, and Ce were analyzed at 60 nanoamps for 60 seconds on peak with 30 seconds on backgrounds and $\mathrm{Cl}$ was measured at the same current for 30 seconds on peak and 15 seconds on backgrounds. Ca, P, and F were analyzed at 10 nanoamps for 10, 40, and 60 seconds, respectively. Accelerating voltage for all analyses was 15 $\mathrm{keV}$. Apatite NMNH 104021 was analyzed at regular intervals to check for analytical drift. Beam diameter was maintained at less than one micron for trace elements and 15 microns for major elements. Three spots were analyzed per apatite crystal. Where there was no obvious chemical zoning or interferences from inclusions within apatite

crystals, the three analyses per crystal were averaged to increase analytical precision.

\section{Table DR1A}

Sample Registry

1 Dickeyville, WI

2 Shakertown, KY

3 Ohio Division of Geological Survey Core \#'s 2626 and 3256

4 Hagan, VA

5 Dolly Ridge, WV

6 State College, PA

7 Whitehall, PA

8 Whitehall, PA

9 Vollen, Norway

10 Vasagård, Bornholm, Denmark

$\begin{array}{lll}\text { Latitude } & \text { Longitude } & \text { Formation } \\ 42.660197^{\circ} & -90.653623^{\circ} & \text { Decorah } \\ 37.762225^{\circ} & -84.843335^{\circ} & \text { Lexington } \\ - & - & \text { Lexington } \\ 36.699982^{\circ} & -83.287978^{\circ} & \text { Eggleston } \\ 38.737248^{\circ} & -79.425366^{\circ} & \text { Dolly Ridge } \\ 40.79688^{\circ} & -77.82192^{\circ} & \text { Salona } \\ 40.660889 \circ & -75.522010^{\circ} & \text { Jacksonburg } \\ & & \\ 59.803589 \circ & 10.486974^{\circ} & \text { Arnestad } \\ 55.056247^{\circ} & 14.887045^{\circ} & \text { Dicellograptus Shale }\end{array}$

Section Description

Emerson et al. 2004

Kolata et al. 1996 and references therein

Kolata et al. 1996 and references therein Kolata et al. 1996 and references therein Kolata et al. 1996 and references therein

Haynes et al. 1995 and references therein

Grahn and Nolvak, 2007 and references therein

\title{
Table DR1B
}

NMNH 104021 and as primary standard for $\mathrm{Ca}, \mathrm{P}$, and F and as secondary standard for trace elements.

Accelerating voltage was $15 \mathrm{keV}$.

\begin{tabular}{|c|c|c|}
\hline EI & Standard & Crystal \\
\hline $\mathrm{Ca}$ & NMNH 104021 Apatite & PET \\
\hline $\mathrm{P}$ & NMNH 104021 Apatite & PET \\
\hline $\mathrm{F}$ & NMNH 104021 Apatite & TAP \\
\hline $\mathrm{Mg}$ & NMNH 111312/444 Olivine & TAP \\
\hline $\mathrm{Cl}$ & NMNH R6600-1 Scapolite & PET \\
\hline $\mathrm{Mn}$ & NMNH 85276 Fayalite & PET \\
\hline $\mathrm{Fe}$ & NMNH 85276 Fayalite & PET \\
\hline Y & NMNH $168499 \mathrm{YPO}_{4}$ & TAP \\
\hline $\mathrm{Ce}$ & NMNH $168484 \mathrm{CePO}_{4}$ & PET \\
\hline
\end{tabular}

\begin{tabular}{|c|c|c|}
\hline $\begin{array}{l}\text { NMNH } \\
104021 \\
\text { Apatite } \\
\text { Analyses }\end{array}$ & & $\begin{array}{l}\% \\
\text { Analytical } \\
\text { Error at } \\
\text { two sigma }\end{array}$ \\
\hline $\mathrm{CaO}$ & 54.42 & 0.5 \\
\hline $\mathrm{P}_{2} \mathrm{O}_{5}$ & 41.41 & 0.5 \\
\hline $\mathrm{F}$ & 3.54 & 3 \\
\hline $\mathrm{MgO}$ & 0.02 & 4 \\
\hline
\end{tabular}




$\begin{array}{lcc}\mathrm{Cl} & 0.42 & 9 \\ \mathrm{MnO} & 0.02 & 13 \\ \mathrm{FeO} & 0.03 & 9 \\ \mathrm{Y}_{2} \mathrm{O}_{3} & 0.08 & 10 \\ \mathrm{Ce}_{2} \mathrm{O}_{3} & 0.42 & 10 \\ & 100.4 \text { sum of measured elements }\end{array}$

$\mathrm{F}$ and $\mathrm{Cl}$ are not converted to oxide form.

No corrections were made because these are not necessary for distinguishing samples (e.g. Samson et al. 1995 and Emerson et al., 2004)

All apatite crystals were mounted in epoxy and oriented so that analyses are perpendicular to the C-axis (see Stormer et al. 1993).

Internal tests show that $\mathrm{F}$ and $\mathrm{Cl}$ analyses can be reproduced.

Relatively stable $\mathrm{Cl}$ values persist in all samples despite coming from different diagenetic and metamorphic conditions.

For example, samples collected in the Valley and Ridge Physiographic Province have been subjected to low-grade metamorphic temperatures (see Repetski et al. 2002), yet retain similar $\mathrm{Cl}$ concentration to those samples that have not been subjected to such conditions.

Also see the statisitics tab for a quatitative description elemental comparions of various samples.

The trace element values for Mg, Mn, Y, and Ce are low compared to the accepted values of Young et al. (1969). This is likely due differing analytical techniques that use non-matrix matched standards.

However, the values are reproducible within our laboratory and corrections can be made when comparing apatite analyses from other EPMA laboratories.

\section{Table DR1C}

Apatite electron probe microanalyses data

Elemental weight percent

Oxide weight percent

\section{Ca}

$$
\begin{array}{llllll}
\mathrm{P} & \mathrm{Mg} \quad \mathrm{Cl} & \mathrm{Fe} & \mathrm{Ce} & \mathrm{Y}
\end{array}
$$

Hagan, Virginia Millbrig \#1 - Top of bed

\begin{tabular}{|c|c|c|c|c|c|c|c|c|}
\hline 37.99 & 3.60 & 17.98 & 0.044 & 0.326 & 0.151 & 0.040 & 0.137 & 0.097 \\
\hline 37.71 & 3.53 & 18.15 & 0.046 & 0.324 & 0.143 & 0.039 & 0.169 & 0.102 \\
\hline 37.93 & 3.51 & 17.91 & 0.047 & 0.326 & 0.146 & 0.048 & 0.149 & 0.089 \\
\hline 38.84 & 3.54 & 18.45 & 0.049 & 0.283 & 0.135 & 0.038 & 0.106 & 0.067 \\
\hline 38.42 & 3.58 & 17.91 & 0.051 & 0.296 & 0.176 & 0.038 & 0.064 & 0.073 \\
\hline 38.32 & 3.56 & 18.43 & 0.054 & 0.291 & 0.150 & 0.037 & 0.125 & 0.058 \\
\hline 38.11 & 3.54 & 18.34 & 0.050 & 0.537 & 0.183 & 0.052 & 0.160 & 0.032 \\
\hline 38.05 & 3.49 & 18.30 & 0.050 & 0.544 & 0.158 & 0.059 & 0.164 & 0.030 \\
\hline 38.44 & 3.57 & 18.49 & 0.050 & 0.564 & 0.083 & 0.048 & 0.154 & 0.000 \\
\hline 38.20 & 3.51 & 18.31 & 0.011 & 0.209 & 0.070 & 0.083 & 0.169 & 0.108 \\
\hline 38.59 & 3.57 & 18.06 & 0.010 & 0.201 & 0.075 & 0.079 & 0.105 & 0.078 \\
\hline 38.46 & 3.48 & 17.95 & 0.009 & 0.211 & 0.105 & 0.080 & 0.112 & 0.111 \\
\hline 38.54 & 3.46 & 18.20 & 0.051 & 0.282 & 0.164 & 0.041 & 0.146 & 0.090 \\
\hline 38.60 & 3.70 & 18.47 & 0.052 & 0.287 & 0.147 & 0.039 & 0.128 & 0.070 \\
\hline 38.18 & 3.45 & 18.37 & 0.049 & 0.284 & 0.169 & 0.039 & 0.121 & 0.067 \\
\hline 38.58 & 3.55 & 18.23 & 0.021 & 0.175 & 0.101 & 0.046 & 0.170 & 0.105 \\
\hline 38.31 & 3.62 & 18.40 & 0.021 & 0.201 & 0.118 & 0.059 & 0.121 & 0.082 \\
\hline 38.44 & 3.47 & 18.12 & 0.021 & 0.171 & 0.122 & 0.046 & 0.148 & 0.088 \\
\hline 38.50 & 3.52 & 18.23 & 0.040 & 0.269 & 0.154 & 0.043 & 0.144 & 0.094 \\
\hline 38.59 & 3.57 & 18.26 & 0.042 & 0.265 & 0.145 & 0.043 & 0.192 & 0.108 \\
\hline & & & & & & & & \\
\hline
\end{tabular}

$\begin{array}{lll}1 & 37.99 \\ 2 & 37.71\end{array}$

438.84

538.42

\begin{tabular}{ll}
7 & 38.11 \\
\hline & 38.05
\end{tabular}

1038.20

1238.46

1338.54

$15 \quad 38.18$

1638.58

$18 \quad 38.44$

2138.93

\begin{abstract}
$\begin{array}{llllllll}3.53 & 18.15 & 0.044 & 0.326 & 0.151 & 0.040 & 0.137 & 0.097\end{array}$ $\begin{array}{lllllllll}3.51 & 17.91 & 0.047 & 0.326 & 0.146 & 0.048 & 0.149 & 0.089\end{array}$ $\begin{array}{lllllllll}3.54 & 18.45 & 0.049 & 0.283 & 0.135 & 0.038 & 0.106 & 0.067\end{array}$ $\begin{array}{lllllllll} & 17.91 & 0.051 & 0.296 & 0.176 & 0.038 & 0.064 & 0.073\end{array}$ $\begin{array}{llllllll}3.54 & 18.34 & 0.050 & 0.291 & 0.150 & 0.037 & 0.125 & 0.058\end{array}$ $\begin{array}{lllllll}8.0 .10 & 0.071\end{array}$ $\begin{array}{llllllll}18.47 & 0.052 & 0.287 & 0.147 & 0.039 & 0.128 & 0.070\end{array}$
\end{abstract}

sum of $\mathrm{Ce}_{2} \mathrm{O}_{3} \quad \mathrm{Y}_{2} \mathrm{O}_{3}$ elements

$\mathrm{CaO} F$

$\mathbf{P}_{\mathbf{2}} \mathbf{O}_{5}$

$\mathrm{Mg}$

FeO Mno

$\begin{array}{lllllllll}53.19 & 3.60 & 41.20 & 0.072 & 0.326 & 0.195 & 0.052 & 0.161 & 0.124\end{array}$ $\begin{array}{lllllllll}52.79 & 3.53 & 41.59 & 0.076 & 0.324 & 0.184 & 0.050 & 0.197 & 0.130\end{array}$ $\begin{array}{lllllllll}53.10 & 3.51 & 41.04 & 0.079 & 0.326 & 0.187 & 0.062 & 0.174 & 0.113\end{array}$ $\begin{array}{lllllllll}54.38 & 3.54 & 42.28 & 0.081 & 0.283 & 0.173 & 0.048 & 0.124 & 0.084\end{array}$ $\begin{array}{lllllllll}53.79 & 3.58 & 41.04 & 0.084 & 0.296 & 0.227 & 0.049 & 0.075 & 0.093\end{array}$ $\begin{array}{lllllllll}53.65 & 3.56 & 42.23 & 0.089 & 0.291 & 0.193 & 0.048 & 0.146 & 0.073\end{array}$ $\begin{array}{llllllllll}53.35 & 3.54 & 42.03 & 0.083 & 0.537 & 0.235 & 0.067 & 0.146 & 0.073 \\ 53.27 & 3.49 & 41.94 & 0.083 & 0.544 & 0.204 & 0.076 & 0.187 & 0.041\end{array}$ $\begin{array}{llllllllll}53.27 & 3.49 & 41.94 & 0.083 & 0.544 & 0.204 & 0.076 & 0.192 & 0.037\end{array}$ $\begin{array}{llllllllll}53.82 & 3.57 & 42.37 & 0.083 & 0.564 & 0.107 & 0.062 & 0.181 & 0.000\end{array}$ $\begin{array}{llllllllll}53.48 & 3.51 & 41.96 & 0.019 & 0.209 & 0.090 & 0.107 & 0.197 & 0.137\end{array}$ $\begin{array}{lllllllll}54.03 & 3.57 & 41.39 & 0.016 & 0.201 & 0.096 & 0.102 & 0.122 & 0.099\end{array}$ $\begin{array}{llllllllll}53.84 & 3.48 & 41.13 & 0.015 & 0.211 & 0.135 & 0.103 & 0.132 & 0.141\end{array}$ $\begin{array}{lllllllll}53.96 & 3.46 & 41.71 & 0.085 & 0.282 & 0.211 & 0.053 & 0.170 & 0.114\end{array}$ $\begin{array}{llllllllll}54.04 & 3.70 & 42.33 & 0.086 & 0.287 & 0.189 & 0.051 & 0.150 & 0.089\end{array}$ $\begin{array}{llllllllll}53.45 & 3.45 & 42.10 & 0.080 & 0.284 & 0.217 & 0.050 & 0.142 & 0.086\end{array}$ $\begin{array}{llllllllll}54.01 & 3.55 & 41.78 & 0.034 & 0.175 & 0.130 & 0.059 & 0.199 & 0.133\end{array}$ $\begin{array}{llllllllll}53.63 & 3.62 & 42.16 & 0.034 & 0.201 & 0.152 & 0.077 & 0.141 & 0.104\end{array}$ $\begin{array}{llllllllll}53.82 & 3.47 & 41.52 & 0.035 & 0.171 & 0.157 & 0.059 & 0.173 & 0.111\end{array}$ $\begin{array}{llllllllll}53.90 & 3.52 & 41.78 & 0.067 & 0.269 & 0.199 & 0.056 & 0.168 & 0.119\end{array}$ $\begin{array}{llllllllll}54.03 & 3.57 & 41.84 & 0.070 & 0.265 & 0.187 & 0.055 & 0.225 & 0.137\end{array}$ $\begin{array}{lllllllll}54.50 & 3.49 & 41.73 & 0.072 & 0.262 & 0.204 & 0.056 & 0.156 & 0.102\end{array}$
98.9

98.9

98.6

101.0

99.2

100.3

100.1

99.8

100.8

99.7

99.6

99.2

100.0

100.9

99.9
100.1

100.1

99.5

100.1

100.4 
2238.46

$\begin{array}{lll}23 & 38.31\end{array}$

$24 \quad 38.19$

$\begin{array}{lll}25 & 38.73\end{array}$

$\begin{array}{ll}26 & 38.39\end{array}$

2738.76

$28 \quad 38.66$

2938.62

$\begin{array}{ll}30 & 39.21\end{array}$

$\begin{array}{ll}31 & 38.51\end{array}$

$\begin{array}{ll}32 & 39.55\end{array}$

$33 \quad 38.44$

$34 \quad 38.79$

$\begin{array}{ll}35 & 38.70\end{array}$

$\begin{array}{lll}36 & 38.37\end{array}$

$37 \quad 38.80$

$38 \quad 38.55$

$\begin{array}{lll}39 & 38.88\end{array}$

$40 \quad 38.65$

$41 \quad 38.49$

$42 \quad 39.03$

$43 \quad 38.49$

$44 \quad 38.12$

$\begin{array}{ll}45 & 6.92\end{array}$

$\begin{array}{ll}46 & 38.87\end{array}$

$\begin{array}{lll}47 & 38.39\end{array}$

$\begin{array}{ll}48 & 38.92\end{array}$

$49 \quad 38.52$

$\begin{array}{ll}50 & 27.20\end{array}$

$51 \quad 38.78$

$\begin{array}{ll}52 & 38.16\end{array}$

$53 \quad 38.18$

$54 \quad 38.33$

$55 \quad 38.48$

$\begin{array}{ll}56 & 38.53\end{array}$

$\begin{array}{lll}57 & 38.57\end{array}$

$\begin{array}{lll}58 & 38.11\end{array}$

$59 \quad 38.55$

$60 \quad 38.43$

$\begin{array}{ll}61 & 38.67\end{array}$

$62 \quad 38.56$

$63 \quad 38.75$

$\begin{array}{ll}64 & 38.47\end{array}$

$65 \quad 38.68$

$\begin{array}{ll}66 & 38.21\end{array}$

$\begin{array}{ll}67 & 38.04\end{array}$

$\begin{array}{ll}68 & 38.31\end{array}$

$\begin{array}{ll}69 & 38.24\end{array}$

$70 \quad 38.64$ $\begin{array}{llllllll}3.52 & 18.31 & 0.045 & 0.461 & 0.150 & 0.043 & 0.208 & 0.073\end{array}$ $\begin{array}{lllllllll}3.55 & 18.17 & 0.047 & 0.456 & 0.164 & 0.037 & 0.174 & 0.078\end{array}$ $\begin{array}{llllllll}3.57 & 18.49 & 0.045 & 0.436 & 0.142 & 0.040 & 0.183 & 0.061\end{array}$ $\begin{array}{lllllllll}3.46 & 18.39 & 0.010 & 0.246 & 0.083 & 0.069 & 0.084 & 0.118\end{array}$ $\begin{array}{llllllll}3.62 & 18.42 & 0.010 & 0.259 & 0.062 & 0.072 & 0.103 & 0.119\end{array}$ $\begin{array}{lllllllll}3.50 & 18.47 & 0.011 & 0.245 & 0.089 & 0.077 & 0.095 & 0.119\end{array}$ $\begin{array}{lllllllll}3.61 & 18.50 & 0.012 & 0.239 & 0.065 & 0.065 & 0.169 & 0.112\end{array}$ $\begin{array}{lllllllll}3.62 & 18.95 & 0.010 & 0.259 & 0.073 & 0.062 & 0.180 & 0.113\end{array}$ $\begin{array}{llllllll}3.55 & 18.28 & 0.019 & 0.239 & 0.113 & 0.061 & 0.184 & 0.136\end{array}$ $\begin{array}{lllllllll}3.47 & 18.56 & 0.019 & 0.242 & 0.121 & 0.054 & 0.224 & 0.140\end{array}$ $\begin{array}{lllllllll}3.69 & 18.21 & 0.017 & 0.259 & 0.108 & 0.060 & 0.216 & 0.167\end{array}$ $\begin{array}{lllllllll}3.67 & 18.48 & 0.007 & 0.206 & 0.053 & 0.080 & 0.066 & 0.073\end{array}$ $\begin{array}{lllllllll}3.61 & 18.34 & 0.008 & 0.218 & 0.069 & 0.079 & 0.061 & 0.061\end{array}$ $\begin{array}{llllllll}3.65 & 17.87 & 0.009 & 0.205 & 0.057 & 0.082 & 0.066 & 0.081\end{array}$ $\begin{array}{llllllll}3.51 & 18.11 & 0.031 & 0.254 & 0.138 & 0.037 & 0.172 & 0.136\end{array}$ $\begin{array}{lllllllll}3.58 & 18.45 & 0.034 & 0.233 & 0.120 & 0.042 & 0.221 & 0.156\end{array}$ $\begin{array}{llllllll}3.50 & 18.57 & 0.037 & 0.240 & 0.136 & 0.036 & 0.273 & 0.199\end{array}$ $\begin{array}{lllllllll}3.41 & 18.64 & 0.023 & 0.283 & 0.117 & 0.045 & 0.156 & 0.084\end{array}$ $\begin{array}{lllllllll}3.61 & 18.19 & 0.024 & 0.272 & 0.126 & 0.046 & 0.157 & 0.083\end{array}$ $\begin{array}{lllllllll}3.58 & 18.50 & 0.026 & 0.282 & 0.117 & 0.044 & 0.173 & 0.082\end{array}$ $\begin{array}{lllllllll}3.54 & 18.60 & 0.028 & 0.229 & 0.139 & 0.038 & 0.177 & 0.088\end{array}$ $\begin{array}{lllllllll}3.48 & 18.40 & 0.026 & 0.231 & 0.103 & 0.037 & 0.150 & 0.081\end{array}$ $\begin{array}{lllllllll}0.48 & 2.25 & 0.026 & 0.243 & 0.138 & 0.039 & 0.162 & 0.083\end{array}$ $\begin{array}{llllllll}3.68 & 18.54 & 0.032 & 0.257 & 0.128 & 0.040 & 0.161 & 0.073\end{array}$ $\begin{array}{llllllll}3.54 & 18.23 & 0.034 & 0.273 & 0.143 & 0.044 & 0.133 & 0.070\end{array}$ $\begin{array}{llllllll}3.69 & 18.21 & 0.032 & 0.282 & 0.122 & 0.044 & 0.139 & 0.071\end{array}$ $\begin{array}{lllllllll}3.27 & 18.52 & 0.126 & 0.213 & 0.251 & 0.049 & 0.037 & 0.041\end{array}$ $\begin{array}{lllllllll}2.47 & 12.18 & 0.124 & 0.227 & 0.215 & 0.041 & 0.041 & 0.040\end{array}$ $\begin{array}{llllllll}3.60 & 18.41 & 0.124 & 0.230 & 0.243 & 0.044 & 0.035 & 0.037\end{array}$ $\begin{array}{lllllllll}3.68 & 18.22 & 0.037 & 0.535 & 0.131 & 0.055 & 0.152 & 0.028\end{array}$ $\begin{array}{lllllllll}3.47 & 18.00 & 0.037 & 0.522 & 0.176 & 0.051 & 0.157 & 0.031\end{array}$ $\begin{array}{lllllllll}3.48 & 18.40 & 0.033 & 0.538 & 0.152 & 0.055 & 0.162 & 0.038\end{array}$ $\begin{array}{lllllllll}3.63 & 18.11 & 0.048 & 0.245 & 0.167 & 0.037 & 0.108 & 0.084\end{array}$ $\begin{array}{lllllllll}3.45 & 18.40 & 0.047 & 0.238 & 0.149 & 0.038 & 0.147 & 0.077\end{array}$ $\begin{array}{llllllll}3.46 & 18.49 & 0.047 & 0.230 & 0.165 & 0.039 & 0.117 & 0.081\end{array}$ $\begin{array}{lllllllll}3.58 & 18.10 & 0.046 & 0.248 & 0.153 & 0.034 & 0.146 & 0.072\end{array}$ $\begin{array}{llllllll}3.30 & 18.17 & 0.066 & 0.562 & 0.263 & 0.063 & 0.129 & 0.029\end{array}$ $\begin{array}{lllllllll}3.66 & 18.17 & 0.044 & 0.519 & 0.172 & 0.064 & 0.154 & 0.024\end{array}$ $\begin{array}{lllllllll}3.44 & 18.20 & 0.045 & 0.505 & 0.196 & 0.070 & 0.171 & 0.019\end{array}$ $\begin{array}{lllllllll}3.53 & 18.38 & 0.046 & 0.295 & 0.140 & 0.035 & 0.144 & 0.075\end{array}$ $\begin{array}{lllllllll}3.71 & 18.25 & 0.045 & 0.293 & 0.162 & 0.039 & 0.131 & 0.084\end{array}$ $\begin{array}{llllllll}3.47 & 18.04 & 0.046 & 0.289 & 0.143 & 0.038 & 0.151 & 0.082\end{array}$ $\begin{array}{llllllll}3.48 & 18.18 & 0.026 & 0.336 & 0.125 & 0.059 & 0.166 & 0.112\end{array}$ $\begin{array}{llllllll}3.57 & 18.10 & 0.024 & 0.355 & 0.118 & 0.063 & 0.250 & 0.148\end{array}$ $\begin{array}{llllllll}3.39 & 18.57 & 0.024 & 0.362 & 0.124 & 0.062 & 0.208 & 0.127\end{array}$ $\begin{array}{llllllll}3.34 & 17.91 & 0.009 & 0.234 & 0.073 & 0.066 & 0.151 & 0.135\end{array}$ $\begin{array}{lllllllll}3.57 & 18.12 & 0.010 & 0.233 & 0.082 & 0.068 & 0.124 & 0.145\end{array}$ $\begin{array}{llllllll}3.64 & 18.45 & 0.009 & 0.244 & 0.063 & 0.065 & 0.161 & 0.143\end{array}$
$53.84 \quad 3.52$

$53.63 \quad 3.55$

$53.47 \quad 3.57$

$54.22 \quad 3.46$

$54.26 \quad 3.50$

$54.12 \quad 3.61$

$54.07 \quad 3.53$

$54.89 \quad 3.62$

$\begin{array}{ll}53.91 & 3.55 \\ 55.37 & 3.47\end{array}$

$55.37 \quad 3.47$

$53.82 \quad 3.69$

$54.31 \quad 3.67$

$54.18 \quad 3.61$

$53.72 \quad 3.65$

54.32

$53.97 \quad 3.58$

$54.43 \quad 3.50$

$54.11 \quad 3.41$

$53.89 \quad 3.61$

$54.64 \quad 3.58$

$53.89 \quad 3.54$

$53.37 \quad 3.48$

$9.69 \quad 0.48$

54.42

$53.75 \quad 3.54$

$54.49 \quad 3.69$

$\begin{array}{ll}53.93 & 3.27\end{array}$

$38.08 \quad 2.47$

$54.29 \quad 3.60$

$53.42 \quad 3.68$

$\begin{array}{ll}53.45 & 3.47\end{array}$

$\begin{array}{ll}53.66 & 3.48 \\ 53.87 & 3.63\end{array}$

$53.87 \quad 3.63$

$54.00 \quad 3.46$

$53.35 \quad 3.58$

$53.97 \quad 3.30$

$53.80 \quad 3.66$

$54.14 \quad 3.44$

$\begin{array}{ll}53.98 & 3.53 \\ 54.25 & 3.71\end{array}$

$\begin{array}{ll}54.25 & 3.71 \\ 53.86 & 3.47\end{array}$

$\begin{array}{ll}53.86 & 3.47 \\ 54.15 & 3.48\end{array}$

$53.49 \quad 3.57$

$53.26 \quad 3.39$
53.63

$53.63 \quad 3.34$

$\begin{array}{ll}53.54 & 3.57 \\ 54.10 & 3.64\end{array}$ $\begin{array}{lllllll}41.96 & 0.075 & 0.461 & 0.192 & 0.055 & 0.243 & 0.093\end{array}$ $\begin{array}{lllllllll}41.64 & 0.077 & 0.456 & 0.211 & 0.048 & 0.204 & 0.099\end{array}$ $\begin{array}{lllllll}42.37 & 0.075 & 0.436 & 0.182 & 0.052 & 0.215 & 0.077\end{array}$ $\begin{array}{llllllll}42.14 & 0.016 & 0.246 & 0.107 & 0.088 & 0.098 & 0.150\end{array}$ $\begin{array}{llllllll}42.21 & 0.017 & 0.259 & 0.079 & 0.093 & 0.121 & 0.151\end{array}$ $\begin{array}{llllllll}42.33 & 0.018 & 0.245 & 0.114 & 0.100 & 0.111 & 0.151\end{array}$ $\begin{array}{llllllll}42.39 & 0.019 & 0.239 & 0.084 & 0.083 & 0.198 & 0.142\end{array}$ $\begin{array}{llllllll}42.39 & 0.019 & 0.239 & 0.084 & 0.083 & 0.198 & 0.142\end{array}$ $\begin{array}{lllllll}4.68 & 0.016 & 0.266 & 0.109 & 0.087 & 0.103 & 0.119\end{array}$ \begin{tabular}{llllllll}
41.48 & 0.016 & 0.266 & 0.109 & 0.087 & 0.103 & 0.119 \\
\hline 1.89 & 0.017 & 0.259 & 0.094 & 0.079 & 0.210 & 0.143
\end{tabular} $\begin{array}{llllllll}41.89 & 0.032 & 0.239 & 0.145 & 0.079 & 0.215 & 0.173\end{array}$ $\begin{array}{lllllll}42.53 & 0.032 & 0.242 & 0.156 & 0.070 & 0.262 & 0.178\end{array}$ $\begin{array}{llllllll}41.73 & 0.028 & 0.259 & 0.139 & 0.078 & 0.253 & 0.212\end{array}$ $\begin{array}{llllllll}42.35 & 0.011 & 0.206 & 0.068 & 0.104 & 0.077 & 0.093\end{array}$ $\begin{array}{llllllll}42.03 & 0.013 & 0.218 & 0.088 & 0.101 & 0.072 & 0.077\end{array}$ $\begin{array}{llllllll}40.95 & 0.015 & 0.205 & 0.073 & 0.106 & 0.077 & 0.102\end{array}$ $\begin{array}{llllllll}41.50 & 0.052 & 0.254 & 0.178 & 0.047 & 0.201 & 0.173\end{array}$ $\begin{array}{llllllll}42.28 & 0.056 & 0.233 & 0.155 & 0.054 & 0.258 & 0.198\end{array}$ $\begin{array}{lllllll}42.55 & 0.061 & 0.233 & 0.155 & 0.054 & 0.258 & 0.198\end{array}$ $\begin{array}{lllllll}2.71 & 0.038 & 0.283 & 0.150 & 0.058 & 0.183 & 0.107\end{array}$ $\begin{array}{llllllll}41.68 & 0.039 & 0.272 & 0.162 & 0.059 & 0.184 & 0.106\end{array}$ $\begin{array}{llllllll}42.39 & 0.044 & 0.282 & 0.150 & 0.056 & 0.203 & 0.105\end{array}$ $\begin{array}{llllllll}42.62 & 0.046 & 0.229 & 0.179 & 0.049 & 0.208 & 0.111\end{array}$ $\begin{array}{llllllll}42.16 & 0.043 & 0.231 & 0.132 & 0.048 & 0.175 & 0.102\end{array}$ $\begin{array}{llllllll}5.16 & 0.044 & 0.243 & 0.177 & 0.050 & 0.190 & 0.105\end{array}$ $\begin{array}{lllllllll}52.49 & 0.054 & 0.257 & 0.164 & 0.052 & 0.189 & 0.093\end{array}$ $\begin{array}{llllllllll}4.79 & 0.054 & 0.257 & 0.164 & 0.052 & 0.189 & 0.093\end{array}$ $\begin{array}{llllllll}41.73 & 0.053 & 0.282 & 0.156 & 0.057 & 0.163 & 0.091\end{array}$ $\begin{array}{llllllll}42.44 & 0.209 & 0.213 & 0.323 & 0.064 & 0.043 & 0.052\end{array}$ $\begin{array}{llllllll}27.91 & 0.205 & 0.227 & 0.277 & 0.053 & 0.048 & 0.051\end{array}$ $\begin{array}{llllllll}42.19 & 0.206 & 0.230 & 0.312 & 0.057 & 0.041 & 0.047\end{array}$ $\begin{array}{llllllll}41.75 & 0.061 & 0.535 & 0.168 & 0.071 & 0.178 & 0.036\end{array}$ $\begin{array}{llllllll}41.25 & 0.061 & 0.522 & 0.227 & 0.065 & 0.183 & 0.039\end{array}$ $\begin{array}{llllllll}42.16 & 0.055 & 0.538 & 0.196 & 0.071 & 0.190 & 0.049\end{array}$ $\begin{array}{llllllll}41.50 & 0.079 & 0.245 & 0.215 & 0.048 & 0.127 & 0.107\end{array}$ $\begin{array}{llllllll}42.50 & 0.079 & 0.245 & 0.215 & 0.048 & 0.127 & 0.107\end{array}$ $\begin{array}{lllllllll}41.16 & 0.077 & 0.238 & 0.191 & 0.049 & 0.172 & 0.098\end{array}$ $\begin{array}{llllllll}42.37 & 0.078 & 0.230 & 0.212 & 0.050 & 0.137 & 0.103\end{array}$ $\begin{array}{llllllll}41.48 & 0.077 & 0.248 & 0.196 & 0.044 & 0.171 & 0.092\end{array}$ $\begin{array}{lllllll}41.64 & 0.110 & 0.562 & 0.338 & 0.082 & 0.151 & 0.036\end{array}$ $\begin{array}{llllllll}41.64 & 0.072 & 0.519 & 0.221 & 0.083 & 0.181 & 0.031\end{array}$ $\begin{array}{llllllll}41.71 & 0.074 & 0.505 & 0.253 & 0.091 & 0.200 & 0.024\end{array}$ $\begin{array}{llllllll}42.12 & 0.077 & 0.295 & 0.180 & 0.045 & 0.168 & 0.096\end{array}$ $\begin{array}{llllllll}41.82 & 0.075 & 0.293 & 0.209 & 0.050 & 0.154 & 0.106\end{array}$ $\begin{array}{llllllll}41.34 & 0.077 & 0.289 & 0.184 & 0.049 & 0.177 & 0.104\end{array}$ $\begin{array}{lllllll}41.66 & 0.043 & 0.336 & 0.160 & 0.076 & 0.194 & 0.142\end{array}$ $\begin{array}{llllllll}4.48 & 0.039 & 0.355 & 0.151 & 0.081 & 0.292 & 0.188\end{array}$ $\begin{array}{llllllll}42.55 & 0.040 & 0.362 & 0.159 & 0.080 & 0.243 & 0.161\end{array}$ $\begin{array}{llllllll}41.04 & 0.015 & 0.234 & 0.094 & 0.085 & 0.177 & 0.171\end{array}$ $\begin{array}{lllllll}41.52 & 0.017 & 0.233 & 0.105 & 0.088 & 0.145 & 0.184\end{array}$ $\begin{array}{lllllll}42.28 & 0.015 & 0.244 & 0.081 & 0.084 & 0.189 & 0.182\end{array}$
00.4

99.9

100.4

100.5

100.3

100.8

100.2

102.3

100.2

100.9

100.4

98.9

100.2

101.6

101.1

100.0

101.5

100.9

99.7

16.1

101.4

99.9

100.7

100.5

69.3

101.0

99.9

99.3

8.

100.4

100.6

99.2

100.2

100.2

100.4

100.5

100.7

99.5

100.2

99.6

100.2

98.8

00.8 
7138.44

$\begin{array}{lll}72 & 38.57\end{array}$

$\begin{array}{ll}73 & 38.34\end{array}$

$\begin{array}{ll}74 & 38.39\end{array}$

$\begin{array}{ll}75 & 38.19\end{array}$

$\begin{array}{lll}76 & 38.95\end{array}$

$\begin{array}{lll}77 & 38.54\end{array}$

$\begin{array}{lll}78 & 38.32\end{array}$

$\begin{array}{ll}79 & 38.69\end{array}$

$\begin{array}{lll}80 & 38.81\end{array}$

8136.01

$82 \quad 38.45$

$83 \quad 38.45$

8438.61

$\begin{array}{lll}85 & 38.73\end{array}$

$\begin{array}{ll}86 & 38.39\end{array}$

$87 \quad 38.75$

$\begin{array}{ll}88 & 38.17\end{array}$

8938.60

$90 \quad 37.62$

9138.42

$92 \quad 38.25$

$93 \quad 38.57$

$94 \quad 38.36$

$\begin{array}{ll}95 & 38.19\end{array}$

$96 \quad 38.58$

$97 \quad 38.37$

$98 \quad 38.16$

$99 \quad 38.29$

$100 \quad 38.43$

$101 \quad 36.52$

$102 \quad 38.29$

10338.44

$104 \quad 38.18$

$105 \quad 38.47$

$106 \quad 38.33$

$107 \quad 38.59$

$108 \quad 37.95$

$109 \quad 37.97$

$110 \quad 38.39$

11138.10

11138.10

$113 \quad 38.28$

$114 \quad 38.14$

$115 \quad 38.49$

$116 \quad 38.25$

$117 \quad 38.20$

$118 \quad 38.12$ $\begin{array}{llllllll}3.43 & 18.30 & 0.030 & 0.363 & 0.147 & 0.044 & 0.144 & 0.055\end{array}$ $\begin{array}{lllllllll}3.44 & 18.38 & 0.026 & 0.369 & 0.136 & 0.056 & 0.174 & 0.053\end{array}$ $\begin{array}{lllllllll}3.54 & 18.05 & 0.029 & 0.358 & 0.157 & 0.060 & 0.178 & 0.052\end{array}$ $\begin{array}{lllllllll}3.49 & 17.83 & 0.020 & 0.253 & 0.121 & 0.050 & 0.325 & 0.196\end{array}$ $\begin{array}{lllllllll}3.64 & 18.36 & 0.021 & 0.252 & 0.134 & 0.049 & 0.215 & 0.138\end{array}$ $\begin{array}{lllllllll}3.61 & 18.53 & 0.021 & 0.254 & 0.103 & 0.043 & 0.284 & 0.181\end{array}$ $\begin{array}{lllllllll}3.55 & 18.28 & 0.022 & 0.231 & 0.123 & 0.050 & 0.182 & 0.111\end{array}$ $\begin{array}{llllllll}3.55 & 18.28 & 0.022 & 0.231 & 0.123 & 0.050 & 0.182 & 0.111\end{array}$ $\begin{array}{lllllllll}3.43 & 18.00 & 0.017 & 0.236 & 0.123 & 0.050 & 0.182 & 0.111\end{array}$ $\begin{array}{llllllll}3.66 & 18.10 & 0.023 & 0.246 & 0.123 & 0.049 & 0.197 & 0.120 \\ 3.46 & 18.43 & 0.028 & 0.220 & 0.123 & 0.059 & 0.186 & 0.091\end{array}$ $\begin{array}{lllllllll}3.46 & 18.43 & 0.028 & 0.220 & 0.123 & 0.059 & 0.186 & 0.091 \\ 3.18 & 16.54 & 0.024 & 0.215 & 0.136 & 0.069 & 0.135 & 0.079\end{array}$ $\begin{array}{llllllll}3.56 & 18.39 & 0.025 & 0.198 & 0.126 & 0.065 & 0.159 & 0.068\end{array}$ $\begin{array}{lllllllll}3.45 & 17.85 & 0.031 & 0.282 & 0.132 & 0.028 & 0.331 & 0.186\end{array}$ $\begin{array}{lllllllll}3.58 & 18.18 & 0.031 & 0.302 & 0.116 & 0.031 & 0.183 & 0.082\end{array}$ $\begin{array}{lllllllll}3.67 & 18.26 & 0.031 & 0.291 & 0.118 & 0.038 & 0.168 & 0.094\end{array}$ $\begin{array}{lllllllll}3.57 & 18.47 & 0.033 & 0.533 & 0.125 & 0.049 & 0.219 & 0.041\end{array}$ $\begin{array}{llllllll}3.40 & 18.20 & 0.034 & 0.537 & 0.143 & 0.048 & 0.193 & 0.042\end{array}$ $\begin{array}{llllllll}3.55 & 18.24 & 0.032 & 0.536 & 0.120 & 0.052 & 0.191 & 0.040\end{array}$ $\begin{array}{lllllllll}3.34 & 17.89 & 0.023 & 0.301 & 0.126 & 0.067 & 0.130 & 0.087\end{array}$ $\begin{array}{lllllllll}3.40 & 17.33 & 0.022 & 0.268 & 0.111 & 0.060 & 0.191 & 0.092\end{array}$

$\begin{array}{llllllll}3.50 & 18.30 & 0.007 & 0.212 & 0.057 & 0.084 & 0.122 & 0.160\end{array}$ $\begin{array}{lllllllll}3.54 & 18.35 & 0.009 & 0.242 & 0.073 & 0.079 & 0.104 & 0.165\end{array}$

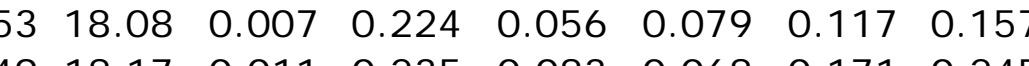
$\begin{array}{lllllll}0.011 & 0.235 & 0.083 & 0.068 & 0.171 & 0.245\end{array}$ $\begin{array}{lllllllll}3.44 & 17.90 & 0.010 & 0.243 & 0.064 & 0.067 & 0.170 & 0.225\end{array}$ $\begin{array}{llllllll}3.56 & 18.36 & 0.007 & 0.217 & 0.057 & 0.068 & 0.192 & 0.325\end{array}$ $\begin{array}{llllllll}3.50 & 18.09 & 0.015 & 0.228 & 0.109 & 0.058 & 0.145 & 0.089\end{array}$ $\begin{array}{llllllll}3.50 & 18.19 & 0.020 & 0.228 & 0.101 & 0.055 & 0.116 & 0.087\end{array}$ $\begin{array}{lllllllll}3.41 & 18.28 & 0.020 & 0.234 & 0.093 & 0.047 & 0.210 & 0.136\end{array}$ $\begin{array}{lllllllll}3.41 & 18.24 & 0.010 & 0.234 & 0.073 & 0.068 & 0.100 & 0.124\end{array}$ $\begin{array}{lllllllll}3.30 & 16.60 & 0.009 & 0.242 & 0.055 & 0.076 & 0.107 & 0.115\end{array}$ $\begin{array}{lllllllll}3.48 & 18.14 & 0.009 & 0.241 & 0.083 & 0.072 & 0.082 & 0.126\end{array}$ $\begin{array}{lllllllll}3.51 & 18.38 & 0.011 & 0.248 & 0.079 & 0.070 & 0.139 & 0.114\end{array}$ $\begin{array}{lllllllll}3.36 & 17.98 & 0.013 & 0.269 & 0.073 & 0.068 & 0.100 & 0.115\end{array}$ $\begin{array}{lllllllll}3.57 & 18.28 & 0.012 & 0.232 & 0.075 & 0.067 & 0.112 & 0.113\end{array}$ $\begin{array}{llllllll}3.54 & 18.03 & 0.009 & 0.235 & 0.061 & 0.077 & 0.129 & 0.181\end{array}$ $\begin{array}{lllllllll}3.29 & 18.24 & 0.011 & 0.228 & 0.071 & 0.070 & 0.147 & 0.170\end{array}$ $\begin{array}{llllllll}3.30 & 17.87 & 0.010 & 0.234 & 0.072 & 0.071 & 0.122 & 0.151\end{array}$ $\begin{array}{lllllllll}3.41 & 17.72 & 0.009 & 0.242 & 0.071 & 0.066 & 0.112 & 0.110\end{array}$ $\begin{array}{lllllllll}3.44 & 17.88 & 0.011 & 0.232 & 0.080 & 0.075 & 0.076 & 0.101\end{array}$ $\begin{array}{lllllllll}3.51 & 18.60 & 0.009 & 0.242 & 0.068 & 0.069 & 0.099 & 0.094\end{array}$ $\begin{array}{lllllllll}3.38 & 18.39 & 0.010 & 0.233 & 0.076 & 0.076 & 0.166 & 0.269\end{array}$ $\begin{array}{lllllllll}3.35 & 18.52 & 0.010 & 0.225 & 0.074 & 0.062 & 0.186 & 0.228\end{array}$ $\begin{array}{llllllll}3.54 & 18.32 & 0.010 & 0.246 & 0.072 & 0.069 & 0.176 & 0.250\end{array}$ $\begin{array}{llllllll}3.50 & 18.49 & 0.014 & 0.228 & 0.097 & 0.050 & 0.156 & 0.110\end{array}$ $\begin{array}{llllllll}3.46 & 18.23 & 0.019 & 0.234 & 0.108 & 0.052 & 0.133 & 0.101\end{array}$ $\begin{array}{lllllllll}3.40 & 18.12 & 0.018 & 0.241 & 0.103 & 0.055 & 0.158 & 0.104\end{array}$ $\begin{array}{llllllll}3.30 & 17.76 & 0.007 & 0.232 & 0.057 & 0.082 & 0.107 & 0.123\end{array}$
$53.82 \quad 3.43$

$54.00 \quad 3.44$

$53.68 \quad 3.54$

$53.75 \quad 3.49$

$\begin{array}{ll}53.47 & 3.64 \\ 54.53 & 3.61\end{array}$

$\begin{array}{ll}4.53 & 3.61 \\ 5 & -1\end{array}$

$53.96 \quad 3.55$

$53.65 \quad 3.43$

$54.17 \quad 3.66$

$54.33 \quad 3.46$

$50.41 \quad 3.18$

$53.83 \quad 3.56$

$\begin{array}{ll}53.83 & 3.45 \\ 54.05 & 3.58\end{array}$

$54.22 \quad 3.67$

$\begin{array}{ll}53.75 & 3.57\end{array}$

$\begin{array}{ll}54.25 & 3.40\end{array}$

$\begin{array}{ll}53.44 & 3.55\end{array}$

$54.04 \quad 3.34$

$52.67 \quad 3.40$

$53.79 \quad 3.50$

$53.55 \quad 3.54$

$54.00 \quad 3.53$

$53.70 \quad 3.49$

$53.47 \quad 3.44$

$54.01 \quad 3.56$

$\begin{array}{ll}53.72 & 3.50\end{array}$

$53.42 \quad 3.50$

$53.61 \quad 3.41$

$53.80-3.41$

$53.61 \quad 3.48$

$53.82 \quad 3.51$

$53.45 \quad 3.36$

$\begin{array}{ll}53.86 & 3.57\end{array}$

$\begin{array}{ll}53.86 & 3.57 \\ 53.66 & 3.54\end{array}$

$\begin{array}{ll}54.03 & 3.29\end{array}$

53.13

$53.16 \quad 3.41$

$\begin{array}{ll}53.75 & 3.44 \\ 53.34 & 3.51\end{array}$

$53.65 \quad 3.38$

$53.59 \quad 3.35$

$53.40 \quad 3.54$

$53.89 \quad 3.50$

$53.55 \quad 3.46$

$53.48 \quad 3.40$

$\begin{array}{ll}53.37 & 3.30\end{array}$ $\begin{array}{llll}41.94 & 0.049 & 0.363 & 0.188\end{array}$

$\begin{array}{llll}42.12 & 0.043 & 0.369 & 0.175\end{array}$

$\begin{array}{llll}41.36 & 0.047 & 0.358 & 0.202\end{array}$

$\begin{array}{llll}40.86 & 0.033 & 0.253 & 0.155\end{array}$

$\begin{array}{llll}42.07 & 0.035 & 0.252 & 0.172\end{array}$

$\begin{array}{llll}42.46 & 0.035 & 0.254 & 0.132\end{array}$

$\begin{array}{llll}41.89 & 0.037 & 0.231 & 0.159\end{array}$

$\begin{array}{llll}41.25 & 0.028 & 0.236 & 0.140\end{array}$

$\begin{array}{llll}41.48 & 0.037 & 0.246 & 0.158\end{array}$

$\begin{array}{llll}42.23 & 0.046 & 0.220 & 0.159\end{array}$

$\begin{array}{lllll}37.90 & 0.039 & 0.215 & 0.175\end{array}$

$\begin{array}{llll}42.14 & 0.042 & 0.198 & 0.162\end{array}$

$\begin{array}{llll}40.90 & 0.052 & 0.282 & 0.170\end{array}$

$\begin{array}{lllll}41.66 & 0.051 & 0.302 & 0.149\end{array}$

$\begin{array}{llll}41.84 & 0.052 & 0.291 & 0.152\end{array}$

$\begin{array}{llll}1.71 & 0.056 & 0.537 & 0.184\end{array}$

$\begin{array}{llll}4.80 & 0.053 & 0.536 & 0.155\end{array}$

$\begin{array}{llll}39.71 & 0.036 & 0.268 & 0.143\end{array}$

$\begin{array}{llll}41.94 & 0.011 & 0.212 & 0.074\end{array}$

$\begin{array}{lllll}42.05 & 0.015 & 0.242 & 0.094\end{array}$

$\begin{array}{llll}41.43 & 0.012 & 0.224 & 0.072\end{array}$

$\begin{array}{lllll}41.64 & 0.019 & 0.235 & 0.106\end{array}$

$\begin{array}{llll}41.02 & 0.017 & 0.243 & 0.082\end{array}$

$\begin{array}{llll}42.07 & 0.011 & 0.217 & 0.074 \\ 41.45 & 0.025 & 0.228 & 0.140\end{array}$

$\begin{array}{llll}41.45 & 0.025 & 0.228 & 0.140\end{array}$

$\begin{array}{llll}41.68 & 0.033 & 0.228 & 0.130\end{array}$

$\begin{array}{lllllll}0.033 & 0.234 & 0.119 & 0.061 & 0.246 & 0.173\end{array}$

$\begin{array}{lllllll}4.80 & 0.017 & 0.234 & 0.094 & 0.088 & 0.117 & 0.157\end{array}$

$\begin{array}{llllllll}38.04 & 0.014 & 0.242 & 0.070 & 0.098 & 0.125 & 0.146\end{array}$

$\begin{array}{lllllll}1.57 & 0.015 & 0.241 & 0.107 & 0.093 & 0.096\end{array}$

$\begin{array}{llllllll}4.12 & 0.017 & 0.248 & 0.102 & 0.091 & 0.163 & 0.145\end{array}$

$\begin{array}{llllllll}41.20 & 0.021 & 0.269 & 0.094 & 0.087 & 0.117 & 0.146\end{array}$

$\begin{array}{llllllll}41.89 & 0.020 & 0.232 & 0.096 & 0.087 & 0.131 & 0.144\end{array}$

$\begin{array}{llllllll}41.32 & 0.015 & 0.235 & 0.078 & 0.099 & 0.151 & 0.230\end{array}$

$\begin{array}{llllllll}41.80 & 0.018 & 0.228 & 0.092 & 0.091 & 0.172 & 0.216\end{array}$

$\begin{array}{llllllll}40.95 & 0.017 & 0.234 & 0.092 & 0.091 & 0.143 & 0.192\end{array}$

$\begin{array}{llllllll}40.61 & 0.015 & 0.242 & 0.091 & 0.085 & 0.131 & 0.139\end{array}$

$\begin{array}{llllllll}40.97 & 0.018 & 0.232 & 0.103 & 0.097 & 0.089 & 0.128\end{array}$

$\begin{array}{llllllll}42.62 & 0.015 & 0.242 & 0.088 & 0.089 & 0.116 & 0.120\end{array}$

$\begin{array}{lllllllll}42.14 & 0.017 & 0.233 & 0.098 & 0.099 & 0.195 & 0.342\end{array}$

$\begin{array}{lllllll}42.44 & 0.016 & 0.233 & 0.098 & 0.09 & 0.195 & 0.342\end{array}$

$\begin{array}{llllllll}42.44 & 0.016 & 0.225 & 0.095 & 0.080 & 0.218 & 0.289 \\ 41.98 & 0.016 & 0.246 & 0.093 & 0.090 & 0.206 & 0.318\end{array}$

$\begin{array}{llllllll}4.98 & 0.016 & 0.246 & 0.093 & 0.090 & 0.206 & 0.318 \\ 41.78 & 0.024 & 0.228 & 0.125 & 0.065 & 0.182 & 0.140\end{array}$

$\begin{array}{llllllll}41.78 & 0.032 & 0.234 & 0.139 & 0.067 & 0.155 & 0.129\end{array}$

$\begin{array}{llllllll}41.52 & 0.029 & 0.241 & 0.132 & 0.071 & 0.185 & 0.132\end{array}$

$\begin{array}{llllllll}40.70 & 0.011 & 0.232 & 0.074 & 0.106 & 0.125 & 0.156\end{array}$
100.1

100.5

99.5

99.2

100.1

101.6

101.6

100.2

99.2

100.2

100.9

92.3

100.3

99.3

100.2

100.6

100.8

00.5

99.2

96.6

100.0

99.9

99.7

99.8

99.

99.3

99.8

99.7

93.2

99.4

00.2

00.0

99.3

97.9

98.8

100.1

99.9

00.5

99.5

99.2

98.1 
11938.21 $\begin{array}{lll}120 & 38.35\end{array}$ $121 \quad 38.16$ $122 \quad 38.35$ $123 \quad 38.82$ $124 \quad 37.99$ $125 \quad 38.21$ $126 \quad 38.13$ $127 \quad 38.33$ $\begin{array}{lll}28 & 38.24\end{array}$ 12938.07 $130 \quad 38.15$ $\begin{array}{lll}131 & 38.20\end{array}$ $\begin{array}{lll}132 & 38.37\end{array}$ $133 \quad 38.57$ $135 \quad 38.00$ $\begin{array}{lll}136 & 38.22\end{array}$ $\begin{array}{lll}37 & 38.27\end{array}$ $138 \quad 37.89$ $139 \quad 37.87$ $140 \quad 37.87$ $141 \quad 37.76$ $142 \quad 37.58$ $\begin{array}{lll}142 & 37.58\end{array}$ $144 \quad 38.10$ $145 \quad 38.05$ $\begin{array}{lll}146 & 38.17\end{array}$ $\begin{array}{lll}147 & 37.76\end{array}$ $148 \quad 38.18$ $\begin{array}{lll}149 & 38.07\end{array}$ $\begin{array}{lll}150 & 37.97\end{array}$ 15137.78 $\begin{array}{lll}152 & 38.02\end{array}$ $153 \quad 38.28$ 15437.76 $155 \quad 37.92$ $156 \quad 37.72$ $157 \quad 37.95$ $\begin{array}{lll}158 & 37.54\end{array}$ $\begin{array}{lll}159 & 37.41\end{array}$ $\begin{array}{lll}160 & 37.35\end{array}$ 16037.35 $\begin{array}{lll}161 & 37.45\end{array}$ $\begin{array}{lll}162 & 37.99\end{array}$ $163 \quad 37.85$ 16438.02 16537.53 $\begin{array}{llllllll}3.43 & 18.16 & 0.007 & 0.223 & 0.054 & 0.077 & 0.115 & 0.111\end{array}$ $\begin{array}{llllllll}3.51 & 18.20 & 0.007 & 0.207 & 0.069 & 0.081 & 0.114 & 0.120\end{array}$ $\begin{array}{llllllll}3.48 & 18.23 & 0.011 & 0.218 & 0.065 & 0.063 & 0.115 & 0.131\end{array}$ $\begin{array}{llllllll}3.39 & 18.03 & 0.009 & 0.231 & 0.074 & 0.069 & 0.086 & 0.127\end{array}$ $\begin{array}{lllllllll}3.46 & 17.94 & 0.008 & 0.221 & 0.066 & 0.069 & 0.132 & 0.134 \\ 3.48 & 18.24 & 0.018 & 0.234 & 0.117 & 0.043 & 0.124 & 0.091\end{array}$ $\begin{array}{llllllllll}3.44 & 18.24 & 0.018 & 0.234 & 0.117 & 0.043 & 0.124 & 0.091\end{array}$ $\begin{array}{lllllllll}3.44 & 18.23 & 0.018 & 0.220 & 0.095 & 0.051 & 0.135 & 0.098\end{array}$ $\begin{array}{lllllllll}3.55 & 18.45 & 0.019 & 0.223 & 0.107 & 0.054 & 0.116 & 0.094\end{array}$ $\begin{array}{lllllllll}3.55 & 18.43 & 0.008 & 0.218 & 0.063 & 0.072 & 0.127 & 0.148\end{array}$ $\begin{array}{lllllllll}3.42 & 18.23 & 0.006 & 0.226 & 0.066 & 0.080 & 0.108 & 0.141\end{array}$ $\begin{array}{lllllllll}3.47 & 18.10 & 0.009 & 0.229 & 0.052 & 0.074 & 0.126 & 0.151\end{array}$ $\begin{array}{lllllllll}3.44 & 18.50 & 0.013 & 0.241 & 0.090 & 0.083 & 0.073 & 0.109\end{array}$ $\begin{array}{lllllllll}3.66 & 18.46 & 0.009 & 0.240 & 0.056 & 0.068 & 0.095 & 0.105\end{array}$ $\begin{array}{lllllllll}3.44 & 18.27 & 0.010 & 0.238 & 0.070 & 0.071 & 0.098 & 0.110\end{array}$ $\begin{array}{llllllll}3.41 & 18.44 & 0.011 & 0.235 & 0.058 & 0.080 & 0.112 & 0.133\end{array}$ $\begin{array}{lllllllll}3.51 & 18.23 & 0.007 & 0.228 & 0.056 & 0.077 & 0.101 & 0.150\end{array}$ $\begin{array}{lllllllll}3.58 & 18.28 & 0.010 & 0.208 & 0.057 & 0.077 & 0.099 & 0.122\end{array}$ $\begin{array}{lllllllll}3.44 & 18.24 & 0.009 & 0.232 & 0.083 & 0.071 & 0.082 & 0.123\end{array}$ $\begin{array}{lllllllll}3.43 & 18.18 & 0.009 & 0.237 & 0.067 & 0.066 & 0.101 & 0.125\end{array}$ $\begin{array}{llllllll}3.45 & 18.41 & 0.008 & 0.230 & 0.088 & 0.074 & 0.080 & 0.129\end{array}$ $\begin{array}{lllllllll}3.54 & 18.41 & 0.012 & 0.210 & 0.063 & 0.072 & 0.119 & 0.109\end{array}$ $\begin{array}{lllllllll}3.44 & 18.49 & 0.011 & 0.231 & 0.063 & 0.072 & 0.111 & 0.104\end{array}$ $\begin{array}{llllllll}3.56 & 17.85 & 0.013 & 0.224 & 0.070 & 0.067 & 0.119 & 0.105\end{array}$ $\begin{array}{lllllllll}3.24 & 18.10 & 0.010 & 0.241 & 0.076 & 0.073 & 0.114 & 0.162\end{array}$ $\begin{array}{lllllllll}3.42 & 17.50 & 0.009 & 0.241 & 0.069 & 0.072 & 0.201 & 0.218\end{array}$ $\begin{array}{lllllllll}3.51 & 18.39 & 0.009 & 0.237 & 0.086 & 0.072 & 0.136 & 0.171\end{array}$ $\begin{array}{llllllll}3.31 & 18.28 & 0.008 & 0.223 & 0.056 & 0.083 & 0.089 & 0.115\end{array}$ $\begin{array}{lllllllll}3.35 & 18.19 & 0.007 & 0.201 & 0.071 & 0.086 & 0.091 & 0.127\end{array}$ $\begin{array}{llllllll}3.52 & 18.36 & 0.008 & 0.212 & 0.061 & 0.083 & 0.091 & 0.111\end{array}$ $\begin{array}{llllllll}3.64 & 18.39 & 0.009 & 0.207 & 0.067 & 0.065 & 0.136 & 0.168\end{array}$ $\begin{array}{lllllllll}3.50 & 18.32 & 0.009 & 0.229 & 0.068 & 0.067 & 0.160 & 0.163\end{array}$ $\begin{array}{lllllllll}3.39 & 18.13 & 0.010 & 0.242 & 0.070 & 0.075 & 0.107 & 0.143\end{array}$ $\begin{array}{lllllllll}3.42 & 18.24 & 0.008 & 0.240 & 0.072 & 0.067 & 0.151 & 0.165\end{array}$ $\begin{array}{lllllllll}3.40 & 18.23 & 0.009 & 0.234 & 0.075 & 0.073 & 0.120 & 0.171\end{array}$ $\begin{array}{lllllllll}3.42 & 18.60 & 0.009 & 0.232 & 0.063 & 0.066 & 0.130 & 0.168\end{array}$ $\begin{array}{llllllll}3.44 & 17.99 & 0.011 & 0.233 & 0.067 & 0.072 & 0.109 & 0.116\end{array}$ $\begin{array}{lllllllll}3.64 & 18.35 & 0.010 & 0.223 & 0.071 & 0.066 & 0.195 & 0.197\end{array}$ $\begin{array}{lllllllll}3.42 & 18.26 & 0.011 & 0.229 & 0.071 & 0.067 & 0.176 & 0.200\end{array}$ $\begin{array}{lllllllll}3.53 & 18.21 & 0.018 & 0.247 & 0.099 & 0.046 & 0.192 & 0.115\end{array}$ $\begin{array}{lllllllll}3.63 & 17.99 & 0.021 & 0.233 & 0.105 & 0.049 & 0.169 & 0.126\end{array}$ $\begin{array}{lllllllll}3.38 & 17.97 & 0.017 & 0.249 & 0.136 & 0.055 & 0.136 & 0.104\end{array}$ $\begin{array}{llllllllll}3.38 & 17.97 & 0.017 & 0.249 & 0.136 & 0.055 & 0.136 & 0.104\end{array}$ $\begin{array}{lllllllll}3.47 & 18.12 & 0.019 & 0.239 & 0.129 & 0.056 & 0.182 & 0.140\end{array}$ $\begin{array}{lllllllll}3.49 & 18.09 & 0.010 & 0.186 & 0.113 & 0.072 & 0.111 & 0.115\end{array}$ $\begin{array}{llllllll}3.43 & 18.30 & 0.008 & 0.177 & 0.076 & 0.076 & 0.094 & 0.110\end{array}$ $\begin{array}{lllllllll}3.53 & 18.09 & 0.009 & 0.193 & 0.064 & 0.070 & 0.126 & 0.114\end{array}$ $\begin{array}{lllllllll}3.49 & 18.06 & 0.008 & 0.231 & 0.063 & 0.081 & 0.153 & 0.207\end{array}$ $\begin{array}{lllllllll}3.47 & 18.19 & 0.009 & 0.232 & 0.068 & 0.086 & 0.146 & 0.207\end{array}$
$53.49 \quad 3.43$

$3.69 \quad 3.51$

53.42

$53.69 \quad 3.39$

$\begin{array}{ll}54.35 & 3.46 \\ 53.19 & 3.48\end{array}$

3.48

53.6

53.54
53.30

53.41

53.48

53.72

54.00

53.68

53.20

53.51
53.58

53.05

53.02

53.02

52.86

52.61

52.8

53.34

53.27
53.44

53.44
52.86

53.45

53.30

53.16

52.89

53.23

53.59

52.86
53.09
52.81

52.81

52.56

52.37

52.29

52.29

52.43
53.19

52.99

53.23

52.54
52.54

52.43 $\begin{array}{llll}41.61 & 0.012 & 0.223 & 0.069\end{array}$

$\begin{array}{lllll}41.71 & 0.011 & 0.207 & 0.089\end{array}$

$\begin{array}{lllll}41.78 & 0.018 & 0.218 & 0.084\end{array}$

$\begin{array}{lllll}41.32 & 0.016 & 0.231 & 0.095\end{array}$

$\begin{array}{lllll}41.11 & 0.013 & 0.221 & 0.085\end{array}$

$\begin{array}{lllll}41.80 & 0.029 & 0.234 & 0.150\end{array}$

41.78

$\begin{array}{lllll}42.78 & 0.012 & 0.218 & 0.081\end{array}$

$\begin{array}{llll}41.48 & 0.014 & 0.229 & 0.066\end{array}$

$\begin{array}{lllll}42.39 & 0.022 & 0.241 & 0.116\end{array}$

$\begin{array}{lllll}42.30 & 0.015 & 0.240 & 0.072\end{array}$

$\begin{array}{lllll}41.87 & 0.016 & 0.238 & 0.090\end{array}$

$\begin{array}{lllll}42.26 & 0.019 & 0.235 & 0.074\end{array}$

$\begin{array}{lllll}41.78 & 0.012 & 0.228 & 0.072\end{array}$

$\begin{array}{lllll}41.89 & 0.016 & 0.208 & 0.073\end{array}$

$\begin{array}{llll}41.80 & 0.015 & 0.232 & 0.107\end{array}$

$\begin{array}{llll}41.66 & 0.015 & 0.237 & 0.086\end{array}$

$\begin{array}{llll}0.013 & 0.230 & 0.113\end{array}$

$\begin{array}{lll}0.021 & 0.210 & 0.081\end{array}$

$\begin{array}{lllll}42.37 & 0.018 & 0.231 & 0.081\end{array}$

$\begin{array}{lllll}40.90 & 0.022 & 0.224 & 0.090\end{array}$

$\begin{array}{lllll}41.48 & 0.017 & 0.241 & 0.098\end{array}$

$\begin{array}{llll}40.10 & 0.015 & 0.241 & 0.089\end{array}$

$\begin{array}{lllll}42.14 & 0.015 & 0.241 & 0.089\end{array}$

$\begin{array}{llll}41.89 & 0.012 & 0.223 & 0.072\end{array}$

$\begin{array}{llll}41.68 & 0.012 & 0.201 & 0.091\end{array}$

$\begin{array}{lllll}42.07 & 0.014 & 0.212 & 0.078\end{array}$

$\begin{array}{lllll}42.14 & 0.015 & 0.207 & 0.086\end{array}$

$\begin{array}{lllll}41.98 & 0.015 & 0.229 & 0.088\end{array}$

$\begin{array}{lllll}41.55 & 0.017 & 0.242 & 0.089\end{array}$

$\begin{array}{lllll}41.80 & 0.013 & 0.240 & 0.093\end{array}$

$\begin{array}{lllll}41.78 & 0.016 & 0.234 & 0.096\end{array}$

$\begin{array}{lllll}42.62 & 0.015 & 0.232 & 0.082\end{array}$

$\begin{array}{llll}41.23 & 0.018 & 0.233 & 0.086\end{array}$

42.05

41.84

41.73

41.36

41.18

40.63

41.52

41.45

41.94

41.45

41.39

41.68 $\begin{array}{lll}.100 & 0.135 & 0.141\end{array}$

$\begin{array}{llll}0.105 & 0.133 & 0.153\end{array}$

$\begin{array}{llll}0.081 & 0.135 & 0.166\end{array}$

$\begin{array}{llll}0.089 & 0.100 & 0.161\end{array}$

$\begin{array}{llll}0.089 & 0.154 & 0.170\end{array}$

$\begin{array}{lll}0.056 & 0.145 & 0.116\end{array}$

$\begin{array}{lll}0.066 & 0.158 & 0.125\end{array}$

$\begin{array}{llll}0.069 & 0.136 & 0.120\end{array}$

$\begin{array}{llll}0.093 & 0.149 & 0.188\end{array}$

$\begin{array}{llll}0.103 & 0.126 & 0.179\end{array}$

$\begin{array}{llll}0.096 & 0.147 & 0.192\end{array}$

$\begin{array}{llll}0.107 & 0.086 & 0.138\end{array}$

$\begin{array}{lll}0.087 & 0.112 & 0.133\end{array}$

$\begin{array}{lll}0.091 & 0.115 & 0.140\end{array}$

$\begin{array}{llll}0.104 & 0.132 & 0.169\end{array}$

$\begin{array}{llll}0.100 & 0.119 & 0.190\end{array}$

$\begin{array}{llll}0.099 & 0.116 & 0.155\end{array}$

$\begin{array}{llll}0.091 & 0.096 & 0.157\end{array}$

$\begin{array}{llll}0.085 & 0.119 & 0.159\end{array}$

$\begin{array}{llll}0.096 & 0.094 & 0.163\end{array}$

$\begin{array}{llll}0.093 & 0.139 & 0.138\end{array}$

$\begin{array}{llll}0.092 & 0.130 & 0.132\end{array}$

$\begin{array}{llll}0.086 & 0.139 & 0.133\end{array}$

$\begin{array}{llll}0.095 & 0.133 & 0.205\end{array}$

$\begin{array}{llll}0.092 & 0.236 & 0.277\end{array}$

$\begin{array}{lll}0.092 & 0.159 & 0.217\end{array}$

$\begin{array}{llll}0.107 & 0.104 & 0.146\end{array}$

$\begin{array}{llll}0.111 & 0.107 & 0.161\end{array}$

$\begin{array}{lll}0.111 & 0.107 & 0.161 \\ 0.108 & 0.107 & 0.141\end{array}$

$\begin{array}{llll}0.084 & 0.160 & 0.213\end{array}$

$\begin{array}{llll}0.086 & 0.187 & 0.207\end{array}$

$\begin{array}{llll}0.097 & 0.126 & 0.181\end{array}$

$\begin{array}{llll}0.087 & 0.177 & 0.210\end{array}$

$\begin{array}{llll}0.094 & 0.140 & 0.217\end{array}$

$\begin{array}{llll}0.085 & 0.152 & 0.214\end{array}$

$\begin{array}{llll}0.093 & 0.128 & 0.147\end{array}$

$\begin{array}{lll}0.085 & 0.228 & 0.250\end{array}$

$\begin{array}{lll}0.086 & 0.206 & 0.254\end{array}$

$\begin{array}{lll}0.059 & 0.225 & 0.146\end{array}$

$\begin{array}{llll}0.064 & 0.197 & 0.160\end{array}$

$\begin{array}{lll}0.062 & 0.239 & 0.150\end{array}$

$\begin{array}{lll}0.071 & 0.159 & 0.132\end{array}$

$\begin{array}{lll}0.059 & 0.286 & 0.177\end{array}$

$\begin{array}{llll}0.072 & 0.213 & 0.177\end{array}$

$\begin{array}{lll}0.093 & 0.130 & 0.146\end{array}$

$\begin{array}{llllll}0.013 & 0.177 & 0.098 & 0.097 & 0.111 & 0.140 \\ 0.014 & 0.193 & 0.082 & 0.091 & 0.148 & 0.145 \\ 0.013 & 0.231 & 0.082 & 0.105 & 0.179 & 0.263\end{array}$

$\begin{array}{llllll}0.013 & 0.177 & 0.098 & 0.097 & 0.111 & 0.140 \\ 0.014 & 0.193 & 0.082 & 0.091 & 0.148 & 0.145 \\ 0.013 & 0.231 & 0.082 & 0.105 & 0.179 & 0.263\end{array}$

$\begin{array}{lllllll}0.013 & 0.231 & 0.082 & 0.105 & 0.179 & 0.263 \\ 0.015 & 0.232 & 0.087 & 0.111 & 0.171 & 0.263\end{array}$
99.2

99.6

99.4

99.7

99.2

99.9

100.2

99.5

00.0

100.1

99.7

100.4

99.7

99.3

99.

99.4

99.4

99.5

98.0

98.

99.1

99.2

99.1

100.0

99.6

98.8

98.2

99.7

99.0

98.2

98.0

97.7

99.1

98.7

98.2

98.3

98.5 
$168 \quad 37.87$ $\begin{array}{lll}169 & 38.13\end{array}$ $170 \quad 37.63$ $171 \quad 37.67$ $172 \quad 37.95$ $173 \quad 37.92$ $\begin{array}{ll}174 & 38.00 \\ 175 & 37.71\end{array}$ $\begin{array}{ll}175 & 37.71 \\ 176 & 37.73\end{array}$ $\begin{array}{lll}176 & 37.73\end{array}$ 17738.02 $178 \quad 38.13$ 17938.65 $180 \quad 38.50$ 18138.42 $182 \quad 38.53$ $\begin{array}{lll}83 & 38.14\end{array}$ $\begin{array}{ll}84 & 38.21\end{array}$ $\begin{array}{lll}85 & 38.29\end{array}$ $86 \quad 38.13$ $187 \quad 38.50$ $\begin{array}{lll}188 & 38.17\end{array}$ $189 \quad 38.13$ 19038.02 19135.81 19238.02

Hagan,

9338.65 19438.25 $195 \quad 38.32$ $196 \quad 37.37$ $197 \quad 38.45$ $198 \quad 38.55$ 19938.08 20037.84 20138.01 20237.11 $203 \quad 38.29$ $204 \quad 38.38$ 20538.18 $206 \quad 37.99$ $207 \quad 38.08$ $208 \quad 38.50$ 20938.18 21138.32 $212 \quad 38.30$ $213 \quad 38.27$ $214 \quad 38.09$ 21538.46 $\begin{array}{llllllll}3.63 & 18.18 & 0.010 & 0.237 & 0.076 & 0.073 & 0.154 & 0.236\end{array}$ $\begin{array}{llllllll}3.40 & 18.23 & 0.010 & 0.216 & 0.064 & 0.070 & 0.109 & 0.141\end{array}$ $\begin{array}{llllllll}3.54 & 18.16 & 0.012 & 0.235 & 0.079 & 0.078 & 0.083 & 0.104\end{array}$ $\begin{array}{lllllllll}3.51 & 18.08 & 0.009 & 0.222 & 0.066 & 0.070 & 0.119 & 0.105\end{array}$ $\begin{array}{lllllllll}3.49 & 17.98 & 0.010 & 0.228 & 0.071 & 0.063 & 0.106 & 0.106\end{array}$ $\begin{array}{lllllllll}3.55 & 18.38 & 0.008 & 0.227 & 0.063 & 0.068 & 0.106 & 0.107\end{array}$ $\begin{array}{llllllll}3.56 & 18.39 & 0.010 & 0.220 & 0.084 & 0.073 & 0.077 & 0.111\end{array}$ $\begin{array}{lllllllll}3.30 & 17.94 & 0.007 & 0.218 & 0.060 & 0.084 & 0.114 & 0.146\end{array}$ $\begin{array}{lllllllll}3.51 & 17.94 & 0.008 & 0.217 & 0.077 & 0.092 & 0.104 & 0.152\end{array}$ $\begin{array}{llllllll}3.63 & 18.05 & 0.009 & 0.224 & 0.057 & 0.088 & 0.115 & 0.156\end{array}$ $\begin{array}{llllllll}3.54 & 18.21 & 0.010 & 0.254 & 0.079 & 0.073 & 0.120 & 0.163\end{array}$ $\begin{array}{lllllllll}3.37 & 18.28 & 0.009 & 0.243 & 0.058 & 0.075 & 0.155 & 0.187 \\ 3.60 & 18.11 & 0.010 & 0.247 & 0.086 & 0.075 & 0.110 & 0.146\end{array}$ $\begin{array}{lllllllll}3.60 & 18.11 & 0.010 & 0.247 & 0.086 & 0.075 & 0.110 & 0.146 \\ 3.46 & 18.22 & 0.010 & 0.223 & 0.059 & 0.070 & 0.196 & 0.152\end{array}$ $\begin{array}{lllllllll}3.53 & 18.18 & 0.009 & 0.223 & 0.086 & 0.077 & 0.109 & 0.119\end{array}$ $\begin{array}{llllllllll}3.43 & 18.38 & 0.010 & 0.216 & 0.057 & 0.075 & 0.125 & 0.107\end{array}$ $\begin{array}{lllllllll}3.43 & 18.38 & 0.010 & 0.216 & 0.057 & 0.075 & 0.125 & 0.107\end{array}$ $\begin{array}{lllllllll}3.49 & 18.05 & 0.008 & 0.232 & 0.060 & 0.071 & 0.076 & 0.104\end{array}$ $\begin{array}{llllllll}3.42 & 18.22 & 0.010 & 0.219 & 0.078 & 0.068 & 0.075 & 0.111\end{array}$ $\begin{array}{llllllll}3.50 & 18.25 & 0.016 & 0.246 & 0.089 & 0.070 & 0.143 & 0.088\end{array}$ $\begin{array}{lllllllll}3.52 & 18.47 & 0.015 & 0.262 & 0.106 & 0.072 & 0.158 & 0.112\end{array}$ $\begin{array}{lllllllll}3.48 & 18.36 & 0.017 & 0.250 & 0.097 & 0.066 & 0.158 & 0.089\end{array}$ $\begin{array}{llllllll}3.58 & 18.12 & 0.009 & 0.230 & 0.072 & 0.068 & 0.131 & 0.181\end{array}$ $\begin{array}{lllllllll}3.28 & 17.11 & 0.010 & 0.237 & 0.063 & 0.078 & 0.116 & 0.147\end{array}$ $\begin{array}{llllllll}3.62 & 18.44 & 0.012 & 0.232 & 0.073 & 0.059 & 0.122 & 0.161\end{array}$ inia Millbrig \#3

$\begin{array}{llllllll}3.38 & 18.38 & 0.019 & 0.246 & 0.107 & 0.050 & 0.129 & 0.089\end{array}$ $\begin{array}{lllllllll}3.52 & 18.51 & 0.017 & 0.236 & 0.122 & 0.048 & 0.115 & 0.099\end{array}$ $\begin{array}{llllllll}3.53 & 18.25 & 0.018 & 0.227 & 0.102 & 0.050 & 0.148 & 0.076\end{array}$ $\begin{array}{lllllllll}3.47 & 17.50 & 0.019 & 0.231 & 0.111 & 0.053 & 0.246 & 0.175\end{array}$ $\begin{array}{lllllllll}3.29 & 18.47 & 0.019 & 0.238 & 0.114 & 0.048 & 0.211 & 0.127\end{array}$ $\begin{array}{lllllllll}3.51 & 18.54 & 0.018 & 0.239 & 0.114 & 0.053 & 0.235 & 0.179\end{array}$ $\begin{array}{lllllllll}3.63 & 18.15 & 0.021 & 0.231 & 0.099 & 0.046 & 0.264 & 0.202\end{array}$ $\begin{array}{lllllllll}3.52 & 18.29 & 0.021 & 0.209 & 0.112 & 0.045 & 0.175 & 0.164\end{array}$ $\begin{array}{lllllllll}3.59 & 18.15 & 0.020 & 0.217 & 0.106 & 0.044 & 0.288 & 0.229\end{array}$ $\begin{array}{lllllllll}3.35 & 17.57 & 0.017 & 0.224 & 0.120 & 0.048 & 0.136 & 0.106\end{array}$ $\begin{array}{llllllll}3.38 & 18.09 & 0.018 & 0.234 & 0.112 & 0.050 & 0.138 & 0.090\end{array}$ $\begin{array}{llllllll}3.41 & 18.22 & 0.018 & 0.246 & 0.123 & 0.055 & 0.117 & 0.090\end{array}$ $\begin{array}{llllllll}3.40 & 18.05 & 0.032 & 0.257 & 0.112 & 0.036 & 0.247 & 0.111\end{array}$ $\begin{array}{lllllllll}3.54 & 18.21 & 0.031 & 0.283 & 0.141 & 0.038 & 0.158 & 0.086\end{array}$ $\begin{array}{llllllll}3.66 & 18.50 & 0.032 & 0.262 & 0.122 & 0.030 & 0.236 & 0.108\end{array}$ $\begin{array}{lllllllll}3.56 & 18.44 & 0.019 & 0.243 & 0.112 & 0.055 & 0.196 & 0.142\end{array}$ $\begin{array}{lllllllll}3.50 & 18.37 & 0.019 & 0.232 & 0.108 & 0.037 & 0.206 & 0.116\end{array}$ $\begin{array}{llllllll}3.62 & 18.32 & 0.019 & 0.235 & 0.115 & 0.049 & 0.158 & 0.114\end{array}$ $\begin{array}{llllllll}3.55 & 18.16 & 0.018 & 0.233 & 0.110 & 0.048 & 0.171 & 0.121\end{array}$ $\begin{array}{lllllllll}3.52 & 18.30 & 0.020 & 0.223 & 0.110 & 0.050 & 0.182 & 0.162\end{array}$ $\begin{array}{lllllllll}3.56 & 18.31 & 0.019 & 0.229 & 0.102 & 0.045 & 0.187 & 0.137\end{array}$ $\begin{array}{lllllllll}3.48 & 18.08 & 0.020 & 0.220 & 0.117 & 0.054 & 0.177 & 0.116\end{array}$ $\begin{array}{lllllllll}3.43 & 18.53 & 0.019 & 0.218 & 0.109 & 0.053 & 0.188 & 0.120\end{array}$
53.38

3.63

$52.68 \quad 3.54$

$52.74 \quad 3.51$

$\begin{array}{ll}53.13 & 3.49 \\ 53.09 & 3.55\end{array}$

$53.09 \quad 3.55$

$53.20 \quad 3.56$

$52.79 \quad 3.30$

$52.82 \quad 3.51$

$53.23 \quad 3.63$

$53.38 \quad 3.54$

$54.11 \quad 3.37$

$\begin{array}{ll}53.90 & 3.60 \\ 53.79 & 3.46 \\ 53.94 & 3.53\end{array}$

$53.94 \quad 3.53$

$53.40 \quad 3.43$

$53.49 \quad 3.37$

$\begin{array}{ll}53.61 & 3.49\end{array}$

$\begin{array}{ll}53.38 & 3.42\end{array}$

$53.90 \quad 3.50$

$53.44 \quad 3.52$

$53.38 \quad 3.48$

$53.23 \quad 3.58$

$50.13 \quad 3.28$

$53.23 \quad 3.62$

$\begin{array}{ll}54.11 & 3.38\end{array}$

$53.55 \quad 3.52$

$\begin{array}{ll}53.65 & 3.53\end{array}$

$\begin{array}{ll}52.32 & 3.47 \\ 53.83 & 3.29\end{array}$

$\begin{array}{ll}53.97 & 3.51\end{array}$

$53.31 \quad 3.63$

$52.98 \quad 3.52$

$53.21 \quad 3.59$

$51.95 \quad 3.35$

$\begin{array}{ll}53.61 & 3.38 \\ 53.73 & 3.41\end{array}$

$53.73 \quad 3.41$

$\begin{array}{ll}53.45 & 3.40\end{array}$

$53.31 \quad 3.66$

$53.90 \quad 3.56$

$53.45 \quad 3.50$

$53.83 \quad 3.62$

$53.65 \quad 3.55$

$53.62 \quad 3.52$

$53.58 \quad 3.56$

$53.33 \quad 3.48$ $\begin{array}{lllllll}41.66 & 0.017 & 0.237 & 0.097 & 0.094 & 0.180 & 0.299\end{array}$ $\begin{array}{llllllll}41.78 & 0.016 & 0.216 & 0.082 & 0.090 & 0.128 & 0.179\end{array}$ $\begin{array}{llllllll}41.61 & 0.020 & 0.235 & 0.102 & 0.101 & 0.097 & 0.132\end{array}$ $\begin{array}{llllllll}41.43 & 0.015 & 0.222 & 0.085 & 0.090 & 0.139 & 0.134\end{array}$ $\begin{array}{llllllll}41.20 & 0.017 & 0.228 & 0.091 & 0.081 & 0.124 & 0.135\end{array}$ $\begin{array}{llllllll}42.12 & 0.013 & 0.227 & 0.082 & 0.088 & 0.124 & 0.136\end{array}$ $\begin{array}{llllllll}42.14 & 0.017 & 0.220 & 0.108 & 0.094 & 0.090 & 0.141\end{array}$ $\begin{array}{llllllll}42.14 & 0.017 & 0.220 & 0.108 & 0.094 & 0.090 & 0.141\end{array}$ $\begin{array}{llllllll}4.11 & 0.011 & 0.218 & 0.077 & 0.108 & 0.134 & 0.186\end{array}$ $\begin{array}{llllllll}41.11 & 0.013 & 0.217 & 0.099 & 0.119 & 0.122 & 0.193\end{array}$ $\begin{array}{llllllll}41.36 & 0.015 & 0.224 & 0.073 & 0.113 & 0.135 & 0.197\end{array}$ $\begin{array}{lllllllll}41.73 & 0.017 & 0.254 & 0.101 & 0.094 & 0.141 & 0.207\end{array}$ $\begin{array}{llllllll}41.89 & 0.015 & 0.243 & 0.074 & 0.097 & 0.181 & 0.237\end{array}$ $\begin{array}{llllllll}41.50 & 0.017 & 0.247 & 0.110 & 0.097 & 0.129 & 0.185\end{array}$ $\begin{array}{llllllll}41.75 & 0.016 & 0.223 & 0.076 & 0.091 & 0.229 & 0.193\end{array}$ $\begin{array}{llllllll}41.66 & 0.016 & 0.223 & 0.111 & 0.099 & 0.128 & 0.151\end{array}$ $\begin{array}{llllllll}42.12 & 0.016 & 0.216 & 0.074 & 0.096 & 0.147 & 0.136\end{array}$ $\begin{array}{llllllll}42.14 & 0.017 & 0.237 & 0.093 & 0.101 & 0.086 & 0.138\end{array}$ $\begin{array}{lllllll}41.36 & 0.013 & 0.232 & 0.078 & 0.092 & 0.089 & 0.133\end{array}$ $\begin{array}{llllllll}4.75 & 0.017 & 0.219 & 0.100 & 0.087 & 0.088 & 0.141\end{array}$ $\begin{array}{llllllll}1.82 & 0.026 & 0.246 & 0.115 & 0.091 & 0.167 & 0.112\end{array}$ $\begin{array}{llllllll}42.33 & 0.025 & 0.262 & 0.137 & 0.093 & 0.186 & 0.142\end{array}$ $\begin{array}{llllllll}42.07 & 0.028 & 0.250 & 0.125 & 0.085 & 0.185 & 0.113\end{array}$ $\begin{array}{llllllll}41.52 & 0.014 & 0.230 & 0.093 & 0.088 & 0.154 & 0.229\end{array}$ $\begin{array}{llllllll}39.21 & 0.017 & 0.237 & 0.081 & 0.100 & 0.136 & 0.186\end{array}$ $\begin{array}{llllllll}42.26 & 0.019 & 0.232 & 0.094 & 0.076 & 0.142 & 0.204\end{array}$

$\begin{array}{lllllll}42.12 & 0.031 & 0.246 & 0.138 & 0.065 & 0.151 & 0.113\end{array}$ $\begin{array}{lllllll}42.42 & 0.028 & 0.236 & 0.157 & 0.062 & 0.135 & 0.125\end{array}$ $\begin{array}{lllllll}41.82 & 0.030 & 0.227 & 0.131 & 0.065 & 0.173 & 0.096\end{array}$ $\begin{array}{llllllll}40.10 & 0.032 & 0.231 & 0.143 & 0.069 & 0.289 & 0.222\end{array}$ $\begin{array}{llllllll}42.33 & 0.032 & 0.238 & 0.146 & 0.061 & 0.247 & 0.162\end{array}$ $\begin{array}{llllllll}42.49 & 0.031 & 0.239 & 0.147 & 0.068 & 0.275 & 0.228\end{array}$ $\begin{array}{llllllll}41.59 & 0.034 & 0.231 & 0.128 & 0.059 & 0.309 & 0.256\end{array}$ $\begin{array}{llllllll}41.91 & 0.035 & 0.209 & 0.144 & 0.058 & 0.205 & 0.208\end{array}$ $\begin{array}{lllllll}41.59 & 0.033 & 0.217 & 0.137 & 0.057 & 0.337 & 0.290\end{array}$ $\begin{array}{llllllll}40.26 & 0.029 & 0.224 & 0.154 & 0.062 & 0.159 & 0.134\end{array}$ $\begin{array}{llllllll}41.45 & 0.030 & 0.234 & 0.143 & 0.064 & 0.162 & 0.114\end{array}$ $\begin{array}{llllllll}41.75 & 0.030 & 0.246 & 0.159 & 0.070 & 0.137 & 0.114\end{array}$ $\begin{array}{llllllll}41.36 & 0.053 & 0.257 & 0.143 & 0.046 & 0.290 & 0.141\end{array}$ $\begin{array}{llllllll}41.73 & 0.051 & 0.283 & 0.182 & 0.049 & 0.185 & 0.109\end{array}$ $\begin{array}{llllllll}42.39 & 0.053 & 0.262 & 0.157 & 0.039 & 0.277 & 0.138\end{array}$ $\begin{array}{llllllll}42.26 & 0.032 & 0.243 & 0.144 & 0.071 & 0.230 & 0.181\end{array}$ $\begin{array}{llllllll}42.10 & 0.032 & 0.232 & 0.139 & 0.048 & 0.241 & 0.147\end{array}$ $\begin{array}{lllllll}41.98 & 0.032 & 0.235 & 0.148 & 0.063 & 0.185 & 0.145\end{array}$ $\begin{array}{lllllll}41.61 & 0.030 & 0.233 & 0.142 & 0.062 & 0.200 & 0.153\end{array}$ $\begin{array}{llllllll}41.94 & 0.033 & 0.223 & 0.142 & 0.065 & 0.213 & 0.205\end{array}$ $\begin{array}{llllllll}41.96 & 0.032 & 0.229 & 0.132 & 0.057 & 0.219 & 0.174\end{array}$ $\begin{array}{llllllll}41.43 & 0.033 & 0.220 & 0.151 & 0.070 & 0.207 & 0.147\end{array}$ $\begin{array}{llllllll}42.46 & 0.031 & 0.218 & 0.140 & 0.069 & 0.220 & 0.153\end{array}$
99.2

99.3

98.5

98.5

99.4

99.6

97.9

98.2

99.5

100.2

99.8

99.8

99.9

99.6

99.7

99.2

100.0

100.1

99.7

99.1

100.4

100.2

99.7

100.3

101.0

99.6

99.3

9.

99.3

100.3

100.6

100.2

99.6

100.0

99.9

00.6 
$216 \quad 38.39$ $217 \quad 38.57$ $218 \quad 38.38$ $219 \quad 37.95$ 22038.05 22138.41 22238.48 $\begin{array}{lll}23 & 38.29\end{array}$ 22438.13 $\begin{array}{lll}225 & 38.31\end{array}$ 22638.02 $227 \quad 38.44$ $228 \quad 38.22$ 22938.08 23038.20 23137.94 $232 \quad 38.02$ $233 \quad 38.54$ 23437.94 $\begin{array}{lll}235 & 38.07\end{array}$ $236 \quad 38.50$ 23738.06 $238 \quad 38.02$ $239 \quad 38.33$ $240 \quad 37.76$ $241 \quad 37.95$ $242 \quad 38.10$ $243 \quad 38.12$ $244 \quad 37.87$ $\begin{array}{lll}245 & 38.37\end{array}$ $246 \quad 37.92$ $247 \quad 37.76$ $248 \quad 38.18$ 24838.18 24938.60 $\begin{array}{lll}250 & 38.40\end{array}$ $\begin{array}{lll}251 & 40.41\end{array}$ $252 \quad 38.35$ $\begin{array}{lll}253 & 38.22\end{array}$ 25438.11 $255 \quad 38.20$ $256 \quad 38.52$ 25738.05 $\begin{array}{lll}258 & 38.19\end{array}$ $\begin{array}{lll}259 & 38.19\end{array}$ $260 \quad 38.16$ 26138.31 $\begin{array}{lll}262 & 38.04\end{array}$ $\begin{array}{lll}263 & 38.17\end{array}$ 26437.82 $\begin{array}{llllllll}3.57 & 18.34 & 0.016 & 0.213 & 0.110 & 0.055 & 0.222 & 0.152\end{array}$ $\begin{array}{llllllll}3.31 & 18.34 & 0.029 & 0.252 & 0.108 & 0.036 & 0.200 & 0.088\end{array}$ $\begin{array}{lllllllll}3.61 & 18.39 & 0.027 & 0.256 & 0.137 & 0.031 & 0.198 & 0.094\end{array}$ $\begin{array}{lllllllll}3.56 & 18.04 & 0.029 & 0.250 & 0.105 & 0.029 & 0.206 & 0.099\end{array}$ $\begin{array}{lllllllll}3.46 & 18.32 & 0.026 & 0.239 & 0.121 & 0.037 & 0.111 & 0.075\end{array}$ $\begin{array}{lllllllll}3.54 & 18.49 & 0.025 & 0.238 & 0.116 & 0.037 & 0.165 & 0.082\end{array}$ $\begin{array}{lllllllll}3.62 & 18.44 & 0.026 & 0.253 & 0.135 & 0.040 & 0.219 & 0.132\end{array}$ $\begin{array}{lllllllll}3.43 & 18.13 & 0.019 & 0.227 & 0.112 & 0.048 & 0.131 & 0.086\end{array}$ $\begin{array}{lllllllll}3.54 & 18.24 & 0.018 & 0.236 & 0.117 & 0.051 & 0.108 & 0.088\end{array}$ $\begin{array}{llllllll}3.40 & 18.51 & 0.018 & 0.226 & 0.102 & 0.053 & 0.152 & 0.111\end{array}$ $\begin{array}{lllllllll}3.45 & 18.36 & 0.013 & 0.234 & 0.087 & 0.065 & 0.105 & 0.073\end{array}$ $\begin{array}{lllllllll}3.62 & 18.07 & 0.011 & 0.232 & 0.077 & 0.058 & 0.126 & 0.080\end{array}$ $\begin{array}{lllllllll}3.45 & 18.05 & 0.012 & 0.223 & 0.103 & 0.057 & 0.155 & 0.084\end{array}$ $\begin{array}{llllllll}3.39 & 17.99 & 0.020 & 0.226 & 0.101 & 0.053 & 0.137 & 0.097\end{array}$ $\begin{array}{lllllllll}3.57 & 18.39 & 0.024 & 0.236 & 0.117 & 0.053 & 0.138 & 0.097\end{array}$ $\begin{array}{llllllll}3.26 & 18.01 & 0.020 & 0.236 & 0.102 & 0.044 & 0.164 & 0.097\end{array}$ $\begin{array}{llllllll}3.51 & 17.86 & 0.019 & 0.224 & 0.121 & 0.044 & 0.137 & 0.095\end{array}$ $\begin{array}{llllllll}3.63 & 18.15 & 0.021 & 0.222 & 0.112 & 0.038 & 0.149 & 0.103\end{array}$ $\begin{array}{lllllllll}3.58 & 18.02 & 0.018 & 0.227 & 0.130 & 0.042 & 0.163 & 0.102\end{array}$ $\begin{array}{llllllll}3.65 & 18.66 & 0.027 & 0.241 & 0.121 & 0.045 & 0.139 & 0.068\end{array}$ $\begin{array}{lllllllll}3.76 & 17.43 & 0.027 & 0.212 & 0.130 & 0.037 & 0.125 & 0.079\end{array}$ $\begin{array}{lllllllll}3.54 & 18.09 & 0.028 & 0.216 & 0.104 & 0.043 & 0.172 & 0.070\end{array}$ $\begin{array}{lllllllll}3.65 & 18.38 & 0.019 & 0.236 & 0.124 & 0.056 & 0.129 & 0.111\end{array}$ $\begin{array}{lllllllll}3.45 & 18.14 & 0.019 & 0.227 & 0.111 & 0.049 & 0.127 & 0.098\end{array}$ $\begin{array}{llllllll}3.49 & 18.22 & 0.020 & 0.234 & 0.116 & 0.052 & 0.110 & 0.099\end{array}$ $\begin{array}{llllllll}3.40 & 18.17 & 0.011 & 0.230 & 0.059 & 0.072 & 0.118 & 0.133\end{array}$ $\begin{array}{llllllll}3.43 & 18.43 & 0.010 & 0.241 & 0.078 & 0.061 & 0.103 & 0.117\end{array}$ $\begin{array}{lllllllll}3.41 & 18.26 & 0.009 & 0.234 & 0.073 & 0.069 & 0.170 & 0.181\end{array}$ $\begin{array}{llllllll}3.35 & 18.65 & 0.020 & 0.238 & 0.119 & 0.060 & 0.121 & 0.093\end{array}$ $\begin{array}{lllllllll}3.45 & 18.12 & 0.017 & 0.256 & 0.109 & 0.047 & 0.167 & 0.101\end{array}$ $\begin{array}{lllllllll}3.43 & 18.19 & 0.019 & 0.236 & 0.114 & 0.049 & 0.119 & 0.094\end{array}$ $\begin{array}{lllllllll}3.59 & 18.24 & 0.023 & 0.234 & 0.112 & 0.040 & 0.148 & 0.080\end{array}$ $\begin{array}{lllllllll}3.60 & 18.36 & 0.028 & 0.228 & 0.120 & 0.035 & 0.156 & 0.085\end{array}$ $\begin{array}{lllllllll}3.44 & 18.19 & 0.025 & 0.229 & 0.126 & 0.038 & 0.128 & 0.075\end{array}$ $\begin{array}{lllllllll}3.06 & 19.65 & 0.020 & 0.217 & 0.115 & 0.046 & 0.110 & 0.091\end{array}$ $\begin{array}{llllllll}3.28 & 20.46 & 0.019 & 0.237 & 0.103 & 0.042 & 0.147 & 0.081\end{array}$ $\begin{array}{lllllllll}3.76 & 18.44 & 0.019 & 0.242 & 0.115 & 0.056 & 0.137 & 0.109\end{array}$ $\begin{array}{llllllll}3.64 & 18.29 & 0.040 & 0.238 & 0.129 & 0.036 & 0.108 & 0.053\end{array}$ $\begin{array}{llllllll}3.50 & 18.16 & 0.042 & 0.249 & 0.168 & 0.045 & 0.082 & 0.058\end{array}$ $\begin{array}{lllllllll}3.63 & 18.04 & 0.043 & 0.249 & 0.141 & 0.038 & 0.129 & 0.053\end{array}$ $\begin{array}{llllllll}3.46 & 18.26 & 0.033 & 0.235 & 0.152 & 0.039 & 0.118 & 0.068\end{array}$ $\begin{array}{lllllllll}3.43 & 18.64 & 0.032 & 0.232 & 0.127 & 0.034 & 0.114 & 0.066\end{array}$ $\begin{array}{lllllllll}3.42 & 18.21 & 0.032 & 0.230 & 0.140 & 0.039 & 0.119 & 0.062\end{array}$ $\begin{array}{llllllll}3.31 & 17.88 & 0.025 & 0.237 & 0.134 & 0.038 & 0.139 & 0.067\end{array}$ $\begin{array}{lllllllll}3.47 & 17.97 & 0.027 & 0.231 & 0.130 & 0.043 & 0.159 & 0.095\end{array}$ $\begin{array}{llllllllll}3.51 & 18.08 & 0.026 & 0.229 & 0.115 & 0.038 & 0.156 & 0.081\end{array}$ $\begin{array}{lllllllll}3.62 & 18.04 & 0.019 & 0.438 & 0.124 & 0.082 & 0.132 & 0.056\end{array}$ $\begin{array}{llllllll}3.41 & 18.56 & 0.020 & 0.448 & 0.118 & 0.089 & 0.151 & 0.055\end{array}$ $\begin{array}{llllllll}3.51 & 17.76 & 0.021 & 0.458 & 0.118 & 0.082 & 0.156 & 0.053\end{array}$
$53.75 \quad 3.57$

$54.00 \quad 3.31$

$53.73 \quad 3.61$

$53.13 \quad 3.56$

$53.27-3.46$

53.87

3.62

$\begin{array}{ll}53.61 & 3.43 \\ 53.38 & 3.54\end{array}$

$53.63 \quad 3.40$

$53.23 \quad 3.45$

$53.82 \quad 3.62$

$53.51 \quad 3.45$

$53.31 \quad 3.39$

$53.48 \quad 3.57$

$53.12 \quad 3.26$

$53.23 \quad 3.51$

$53.96 \quad 3.63$

$53.12 \quad 3.58$

$53.30 \quad 3.65$

$53.90 \quad 3.76$

$53.28 \quad 3.54$

$53.23 \quad 3.65$

$53.66 \quad 3.45$

$52.86 \quad 3.49$

$53.13 \quad 3.40$

$53.34 \quad 3.43$

$\begin{array}{ll}53.37 & 3.41\end{array}$

$\begin{array}{ll}53.02 & 3.35\end{array}$

$\begin{array}{ll}53.72 & 3.45 \\ 53.09 & 3.43\end{array}$

$\begin{array}{ll}53.09 & 3.43 \\ 52.86 & 3.59\end{array}$

$53.45 \quad 3.60$

$53.45 \quad 3.60$

$53.76 \quad 3.06$

$56.57 \quad 3.28$

$53.69 \quad 3.76$

$53.51 \quad 3.64$

$53.35 \quad 3.50$

$\begin{array}{ll}53.48 & 3.63 \\ 53.93 & 3.46\end{array}$

$\begin{array}{ll}53.93 & 3.46 \\ 53.27 & 3.33\end{array}$

$53.47 \quad 3.42$

$\begin{array}{ll}53.47 & 3.31\end{array}$

$53.42 \quad 3.47$

$53.63 \quad 3.51$

$53.26 \quad 3.62$

$\begin{array}{ll}53.44 & 3.41 \\ 52.95 & 3.51\end{array}$ $\begin{array}{llllllll}2.03 & 0.027 & 0.213 & 0.141 & 0.071 & 0.260 & 0.193\end{array}$ $\begin{array}{llllllll}42.03 & 0.047 & 0.252 & 0.139 & 0.047 & 0.234 & 0.112\end{array}$ $\begin{array}{lllllll}42.14 & 0.045 & 0.256 & 0.176 & 0.040 & 0.231 & 0.119\end{array}$ $\begin{array}{llllllll}41.34 & 0.048 & 0.250 & 0.135 & 0.038 & 0.242 & 0.126\end{array}$ $\begin{array}{llllllll}41.98 & 0.044 & 0.239 & 0.156 & 0.047 & 0.130 & 0.095\end{array}$ $\begin{array}{llllllll}42.37 & 0.042 & 0.238 & 0.150 & 0.048 & 0.194 & 0.104\end{array}$ $\begin{array}{lllllllll}42.26 & 0.043 & 0.253 & 0.174 & 0.052 & 0.257 & 0.168\end{array}$ $\begin{array}{lllllllll}42.37 & 0.042 & 0.238 & 0.150 & 0.048 & 0.194 & 0.104\end{array}$ $\begin{array}{lllllll}4.55 & 0.031 & 0.227 & 0.144 & 0.062 & 0.153 & 0.110\end{array}$ $\begin{array}{lllllllll}4.80 & 0.029 & 0.236 & 0.150 & 0.066 & 0.127 & 0.112\end{array}$ $\begin{array}{llllllll}42.42 & 0.030 & 0.226 & 0.131 & 0.068 & 0.177 & 0.141\end{array}$ $\begin{array}{lllllllll}42.07 & 0.022 & 0.234 & 0.112 & 0.084 & 0.123 & 0.093\end{array}$ $\begin{array}{llllllll}41.41 & 0.019 & 0.232 & 0.100 & 0.075 & 0.148 & 0.101\end{array}$ $\begin{array}{llllllll}41.36 & 0.020 & 0.223 & 0.133 & 0.074 & 0.181 & 0.107\end{array}$ $\begin{array}{llllllll}41.23 & 0.034 & 0.226 & 0.130 & 0.069 & 0.160 & 0.123\end{array}$ $\begin{array}{llllllll}42.14 & 0.039 & 0.236 & 0.150 & 0.069 & 0.161 & 0.124\end{array}$ $\begin{array}{lllllllll}41.27 & 0.034 & 0.236 & 0.132 & 0.057 & 0.192 & 0.123\end{array}$ $\begin{array}{lllllll}40.93 & 0.032 & 0.224 & 0.155 & 0.057 & 0.160 & 0.121\end{array}$ $\begin{array}{llllllll}41.59 & 0.034 & 0.222 & 0.143 & 0.049 & 0.175 & 0.131\end{array}$ $\begin{array}{lllllll}1.29 & 0.029 & 0.227 & 0.167 & 0.055 & 0.191 & 0.130\end{array}$ $\begin{array}{llllllll}42.76 & 0.044 & 0.241 & 0.155 & 0.059 & 0.163 & 0.086\end{array}$ $\begin{array}{llllllll}39.94 & 0.044 & 0.212 & 0.167 & 0.047 & 0.147 & 0.101\end{array}$ $\begin{array}{llllllll}41.45 & 0.046 & 0.216 & 0.133 & 0.055 & 0.201 & 0.089\end{array}$ $\begin{array}{llllllll}42.12 & 0.032 & 0.236 & 0.159 & 0.073 & 0.151 & 0.141\end{array}$ $\begin{array}{llllllll}41.57 & 0.031 & 0.227 & 0.143 & 0.063 & 0.148 & 0.125\end{array}$ $\begin{array}{lllllll}41.75 & 0.034 & 0.234 & 0.149 & 0.067 & 0.128 & 0.125\end{array}$ $\begin{array}{lllllll}41.64 & 0.019 & 0.230 & 0.075 & 0.093 & 0.139 & 0.169\end{array}$ $\begin{array}{llllllll}42.23 & 0.016 & 0.241 & 0.100 & 0.079 & 0.121 & 0.149\end{array}$ $\begin{array}{llllllll}41.84 & 0.015 & 0.234 & 0.094 & 0.088 & 0.200 & 0.230\end{array}$ $\begin{array}{llllllll}42.74 & 0.033 & 0.238 & 0.153 & 0.077 & 0.142 & 0.118\end{array}$ $\begin{array}{llllllll}41.52 & 0.028 & 0.256 & 0.141 & 0.061 & 0.195 & 0.128\end{array}$ $\begin{array}{llllllll}41.68 & 0.032 & 0.236 & 0.147 & 0.063 & 0.139 & 0.120\end{array}$ $\begin{array}{llllllll}41.80 & 0.037 & 0.234 & 0.144 & 0.051 & 0.173 & 0.102\end{array}$ $\begin{array}{llllllll}42.07 & 0.046 & 0.228 & 0.155 & 0.045 & 0.183 & 0.108\end{array}$ $\begin{array}{llllllll}41.68 & 0.041 & 0.229 & 0.162 & 0.050 & 0.150 & 0.095\end{array}$ $\begin{array}{llllllll}45.03 & 0.033 & 0.217 & 0.148 & 0.059 & 0.129 & 0.115\end{array}$ $\begin{array}{llllllll}46.89 & 0.032 & 0.237 & 0.133 & 0.054 & 0.173 & 0.103\end{array}$ $\begin{array}{llllllll}42.26 & 0.032 & 0.242 & 0.148 & 0.073 & 0.161 & 0.139\end{array}$ $\begin{array}{llllllll}41.91 & 0.066 & 0.238 & 0.165 & 0.046 & 0.126 & 0.067\end{array}$ $\begin{array}{llllllll}41.61 & 0.069 & 0.249 & 0.216 & 0.058 & 0.096 & 0.073\end{array}$ $\begin{array}{llllllll}41.34 & 0.070 & 0.249 & 0.181 & 0.049 & 0.151 & 0.067\end{array}$ $\begin{array}{llllllll}41.84 & 0.055 & 0.235 & 0.196 & 0.051 & 0.139 & 0.086\end{array}$ $\begin{array}{lllllll}42.71 & 0.053 & 0.232 & 0.163 & 0.043 & 0.134 & 0.083\end{array}$ $\begin{array}{lllllll}41.73 & 0.053 & 0.230 & 0.180 & 0.051 & 0.139 & 0.078\end{array}$ $\begin{array}{llllllll}40.97 & 0.042 & 0.237 & 0.172 & 0.048 & 0.163 & 0.085\end{array}$ $\begin{array}{llllllll}41.18 & 0.044 & 0.231 & 0.168 & 0.055 & 0.186 & 0.120\end{array}$ $\begin{array}{llllllll}41.43 & 0.043 & 0.229 & 0.147 & 0.049 & 0.183 & 0.103\end{array}$ $\begin{array}{llllllll}41.34 & 0.031 & 0.438 & 0.160 & 0.106 & 0.155 & 0.071\end{array}$ $\begin{array}{lllllll}42.53 & 0.033 & 0.448 & 0.152 & 0.115 & 0.177 & 0.070\end{array}$ $\begin{array}{lllllll}40.70 & 0.035 & 0.458 & 0.152 & 0.106 & 0.182 & 0.067\end{array}$
100.2

100.2

100.4

100.5

100.7

99.3

99.4

99.4

99.5

99.1

98.7

100.0

98.4

99.

98.8

100.5

98.3

99.0

99.8

98.9

99.7

99.5

99.5

98.9

99.0

99.9

102.6

107.5

99.8

99.2

99.2

100.0

100.0

99.3

98.5

99.3

99.2 
26538.07 26638.26 26738.61 $\begin{array}{lll}268 & 38.30\end{array}$ $269 \quad 38.22$ $270 \quad 38.66$ 27038.66 $\begin{array}{ll}271 & 38.27 \\ 272 & 38.39\end{array}$ $\begin{array}{ll}272 & 38.39\end{array}$ $\begin{array}{lll}273 & 38.45\end{array}$ $274 \quad 38.49$ $275 \quad 38.13$ $276 \quad 38.94$ $277 \quad 38.54$ $278 \quad 38.42$ $\begin{array}{ll}279 & 38.39\end{array}$ $\begin{array}{lll}280 & 38.27 \\ 281 & 38.27\end{array}$ $\begin{array}{lll}281 & 38.27\end{array}$ $\begin{array}{lll}282 & 38.47\end{array}$ $\begin{array}{lll}283 & 38.48\end{array}$ $284 \quad 38.58$ $285 \quad 38.74$ $286 \quad 38.42$ $\begin{array}{lll}287 & 38.24\end{array}$ $288 \quad 38.70$ $\begin{array}{lll}290 & 38.52\end{array}$ $\begin{array}{lll}91 & 38.27\end{array}$ $292 \quad 38.29$ $293 \quad 38.04$ 29438.49 $295 \quad 38.04$ 29638.26 29738.07

Hagan, 29838.83 29938.53 30038.12 30137.98 30238.55 $303 \quad 38.01$ 30438.02 30537.84 $\begin{array}{lll}306 & 39.29\end{array}$ 30.48 $308 \quad 38.08$ $\begin{array}{lll}309 & 38.21\end{array}$ $310 \quad 38.18$ 31138.06 31238.42 $\begin{array}{llllllll}3.54 & 18.22 & 0.023 & 0.226 & 0.115 & 0.035 & 0.186 & 0.138\end{array}$ $\begin{array}{llllllll}3.54 & 18.47 & 0.022 & 0.224 & 0.119 & 0.045 & 0.139 & 0.105\end{array}$ $\begin{array}{llllllll}3.31 & 18.60 & 0.024 & 0.204 & 0.103 & 0.036 & 0.174 & 0.079\end{array}$ $\begin{array}{lllllllll}3.44 & 18.26 & 0.019 & 0.231 & 0.109 & 0.057 & 0.186 & 0.122\end{array}$ $\begin{array}{lllllllll}3.43 & 18.35 & 0.018 & 0.237 & 0.104 & 0.050 & 0.233 & 0.144\end{array}$ $\begin{array}{lllllllll}3.43 & 18.15 & 0.018 & 0.234 & 0.115 & 0.046 & 0.272 & 0.184\end{array}$ $\begin{array}{llllllll}3.39 & 18.21 & 0.043 & 0.260 & 0.153 & 0.044 & 0.138 & 0.067\end{array}$ $\begin{array}{lllllllll}3.54 & 18.01 & 0.040 & 0.273 & 0.168 & 0.044 & 0.125 & 0.078\end{array}$ $\begin{array}{llllllll}3.70 & 18.18 & 0.039 & 0.282 & 0.139 & 0.051 & 0.134 & 0.064\end{array}$ $\begin{array}{llllllll}3.57 & 18.38 & 0.023 & 0.228 & 0.106 & 0.039 & 0.141 & 0.088\end{array}$ $\begin{array}{lllllllll}3.40 & 18.22 & 0.023 & 0.223 & 0.116 & 0.041 & 0.159 & 0.108\end{array}$ $\begin{array}{lllllllll}3.67 & 18.40 & 0.025 & 0.230 & 0.121 & 0.038 & 0.157 & 0.099\end{array}$ $\begin{array}{lllllllll}3.55 & 18.24 & 0.019 & 0.229 & 0.106 & 0.055 & 0.133 & 0.094\end{array}$ $\begin{array}{lllllllll}3.44 & 17.96 & 0.020 & 0.232 & 0.123 & 0.059 & 0.125 & 0.086\end{array}$ $\begin{array}{lllllllll}3.40 & 18.46 & 0.018 & 0.225 & 0.103 & 0.052 & 0.169 & 0.093\end{array}$ $\begin{array}{lllllllll}3.51 & 18.56 & 0.028 & 0.227 & 0.140 & 0.038 & 0.317 & 0.207\end{array}$ $\begin{array}{lllllllll}3.39 & 18.46 & 0.027 & 0.237 & 0.111 & 0.032 & 0.305 & 0.174\end{array}$ $\begin{array}{llllllll}3.70 & 18.35 & 0.027 & 0.231 & 0.135 & 0.037 & 0.285 & 0.193\end{array}$ $\begin{array}{llllllll}3.39 & 18.55 & 0.028 & 0.233 & 0.128 & 0.033 & 0.361 & 0.190\end{array}$ $\begin{array}{llllllll}3.55 & 18.26 & 0.028 & 0.233 & 0.132 & 0.032 & 0.298 & 0.179\end{array}$ $\begin{array}{lllllllll}3.57 & 18.22 & 0.025 & 0.252 & 0.109 & 0.034 & 0.274 & 0.149\end{array}$ $\begin{array}{lllllllll}3.51 & 18.06 & 0.026 & 0.324 & 0.128 & 0.043 & 0.205 & 0.091\end{array}$ $\begin{array}{lllllllll}3.39 & 18.19 & 0.031 & 0.318 & 0.131 & 0.040 & 0.256 & 0.126\end{array}$ $\begin{array}{lllllllll}3.45 & 18.10 & 0.027 & 0.303 & 0.127 & 0.044 & 0.192 & 0.101\end{array}$ $\begin{array}{llllllll}3.58 & 18.10 & 0.018 & 0.224 & 0.111 & 0.038 & 0.199 & 0.114\end{array}$ $\begin{array}{lllllllll}3.46 & 18.44 & 0.019 & 0.228 & 0.119 & 0.040 & 0.166 & 0.111\end{array}$ $\begin{array}{llllllll}3.42 & 18.05 & 0.020 & 0.220 & 0.106 & 0.035 & 0.239 & 0.112\end{array}$ $\begin{array}{lllllllll}3.44 & 18.26 & 0.031 & 0.266 & 0.135 & 0.040 & 0.249 & 0.105\end{array}$ $\begin{array}{llllllll}3.42 & 18.05 & 0.031 & 0.253 & 0.125 & 0.030 & 0.270 & 0.106\end{array}$ $\begin{array}{llllllll}3.39 & 18.44 & 0.030 & 0.260 & 0.129 & 0.030 & 0.273 & 0.120\end{array}$ $\begin{array}{lllllllll}3.51 & 17.88 & 0.019 & 0.232 & 0.110 & 0.051 & 0.141 & 0.094\end{array}$ $\begin{array}{lllllllll}3.50 & 18.19 & 0.019 & 0.234 & 0.112 & 0.054 & 0.159 & 0.100\end{array}$ $\begin{array}{llllllll}3.57 & 18.11 & 0.017 & 0.251 & 0.107 & 0.053 & 0.174 & 0.112\end{array}$ ginia Millbrig \#4

$\begin{array}{llllllll}3.58 & 18.14 & 0.026 & 0.240 & 0.114 & 0.038 & 0.191 & 0.110\end{array}$ $\begin{array}{lllllllll}3.64 & 18.16 & 0.021 & 0.228 & 0.104 & 0.037 & 0.183 & 0.084\end{array}$ $\begin{array}{llllllll}3.40 & 17.76 & 0.025 & 0.245 & 0.133 & 0.040 & 0.199 & 0.118\end{array}$ $\begin{array}{lllllllll}3.58 & 17.62 & 0.017 & 0.242 & 0.105 & 0.050 & 0.173 & 0.112\end{array}$ $\begin{array}{lllllllll}3.53 & 17.97 & 0.018 & 0.250 & 0.105 & 0.054 & 0.152 & 0.107\end{array}$ $\begin{array}{lllllllll}3.42 & 18.17 & 0.018 & 0.239 & 0.109 & 0.046 & 0.167 & 0.093\end{array}$ $\begin{array}{lllllllll}3.48 & 18.16 & 0.028 & 0.241 & 0.126 & 0.045 & 0.162 & 0.083\end{array}$ $\begin{array}{lllllllll}3.42 & 17.85 & 0.025 & 0.233 & 0.112 & 0.034 & 0.158 & 0.092\end{array}$ $\begin{array}{llllllll}3.47 & 18.16 & 0.025 & 0.237 & 0.122 & 0.032 & 0.159 & 0.095\end{array}$ $\begin{array}{llllllll}3.62 & 18.15 & 0.018 & 0.229 & 0.103 & 0.046 & 0.160 & 0.075\end{array}$ $\begin{array}{llllllll}3.38 & 18.25 & 0.021 & 0.227 & 0.120 & 0.054 & 0.123 & 0.078\end{array}$ $\begin{array}{llllllll}3.56 & 18.62 & 0.018 & 0.230 & 0.113 & 0.050 & 0.151 & 0.081\end{array}$ $\begin{array}{lllllllll}3.53 & 18.00 & 0.021 & 0.228 & 0.117 & 0.049 & 0.126 & 0.094\end{array}$ $\begin{array}{lllllllll}3.47 & 18.43 & 0.018 & 0.209 & 0.107 & 0.046 & 0.143 & 0.087\end{array}$ $\begin{array}{lllllllll}3.35 & 18.15 & 0.017 & 0.226 & 0.106 & 0.043 & 0.190 & 0.107\end{array}$ $\begin{array}{ll}5.56 & 3.54 \\ 53.5 & -1\end{array}$

$54.05 \quad 3.31$

$53.62 \quad 3.44$

$\begin{array}{ll}53.51 & 3.43 \\ 54.12 & 3.43 \\ 53.58 & 3.39\end{array}$

$53.58 \quad 3.39$

53.753 .54

$53.83 \quad 3.70$

$53.89 \quad 3.57$

$53.38 \quad 3.40$

$54.52 \quad 3.67$

$\begin{array}{ll}53.96 & 3.55 \\ 53.79 & 3.44 \\ 53.75 & 3.40\end{array}$

$\begin{array}{ll}53.75 & 3.40\end{array}$

$53.58 \quad 3.51$

$53.58 \quad 3.39$

$53.86 \quad 3.70$

$53.87 \quad 3.39$

$54.01 \quad 3.55$

$54.24 \quad 3.57$

$53.79 \quad 3.51$

$53.54 \quad 3.39$

$54.18 \quad 3.45$

$54.17 \quad 3.58$

$53.93 \quad 3.46$

$\begin{array}{ll}53.58 & 3.42\end{array}$

$\begin{array}{ll}53.61 & 3.44\end{array}$

$\begin{array}{ll}53.26 & 3.42\end{array}$

$53.89 \quad 3.39$

$53.26 \quad 3.51$

$54.36 \quad 3.58$

$53.94 \quad 3.64$

$53.37 \quad 3.40$

$53.17 \quad 3.58$

$53.97 \quad 3.53$

$53.21 \quad 3.42$

$53.23 \quad 3.48$

$52.98 \quad 3.42$

$\begin{array}{ll}55.01 & 3.47\end{array}$

$53.87 \quad 3.62$

$53.31 \quad 3.38$

$53.49 \quad 3.56$

$53.45 \quad 3.53$

$\begin{array}{ll}53.28 & 3.47 \\ 53.79 & 3.35\end{array}$ $\begin{array}{lllllll}41.75 & 0.038 & 0.226 & 0.148 & 0.045 & 0.217 & 0.175\end{array}$ $\begin{array}{lllllll}42.33 & 0.037 & 0.224 & 0.154 & 0.058 & 0.162 & 0.133\end{array}$ $\begin{array}{lllllll}42.62 & 0.039 & 0.204 & 0.133 & 0.047 & 0.203 & 0.100\end{array}$ $\begin{array}{llllllll}41.84 & 0.032 & 0.231 & 0.141 & 0.074 & 0.218 & 0.155\end{array}$ $\begin{array}{llllllll}42.05 & 0.029 & 0.237 & 0.134 & 0.064 & 0.273 & 0.183\end{array}$ $\begin{array}{llllllll}41.59 & 0.030 & 0.234 & 0.148 & 0.059 & 0.318 & 0.234\end{array}$ $\begin{array}{llllllll}41.73 & 0.071 & 0.260 & 0.196 & 0.057 & 0.161 & 0.086\end{array}$ $\begin{array}{llllllll}41.27 & 0.066 & 0.273 & 0.216 & 0.057 & 0.146 & 0.099\end{array}$ $\begin{array}{llllllll}41.66 & 0.064 & 0.282 & 0.178 & 0.066 & 0.157 & 0.082\end{array}$ $\begin{array}{llllllll}42.12 & 0.038 & 0.228 & 0.137 & 0.050 & 0.165 & 0.111\end{array}$ $\begin{array}{llllllll}4.75 & 0.037 & 0.223 & 0.149 & 0.052 & 0.186 & 0.138\end{array}$ $\begin{array}{llllllll}42.16 & 0.041 & 0.230 & 0.156 & 0.049 & 0.183 & 0.126\end{array}$ $\begin{array}{lllllll}41.80 & 0.032 & 0.229 & 0.137 & 0.071 & 0.155 & 0.119\end{array}$ $\begin{array}{llllllll}41.16 & 0.033 & 0.232 & 0.159 & 0.077 & 0.147 & 0.109\end{array}$ $\begin{array}{llllllll}42.30 & 0.029 & 0.225 & 0.132 & 0.068 & 0.198 & 0.118\end{array}$ $\begin{array}{lllllllll}42.53 & 0.046 & 0.227 & 0.180 & 0.049 & 0.371 & 0.262\end{array}$ $\begin{array}{llllllll}42.30 & 0.045 & 0.237 & 0.142 & 0.041 & 0.357 & 0.221\end{array}$ $\begin{array}{llllllll}42.05 & 0.045 & 0.231 & 0.174 & 0.047 & 0.334 & 0.245\end{array}$ $\begin{array}{llllllll}42.51 & 0.046 & 0.233 & 0.164 & 0.043 & 0.423 & 0.241\end{array}$ $\begin{array}{llllllll}41.84 & 0.047 & 0.233 & 0.170 & 0.041 & 0.349 & 0.227\end{array}$ $\begin{array}{lllllllll}41.75 & 0.042 & 0.252 & 0.140 & 0.044 & 0.321 & 0.189\end{array}$ $\begin{array}{llllllll}41.39 & 0.043 & 0.324 & 0.164 & 0.056 & 0.241 & 0.115\end{array}$ $\begin{array}{llllllll}41.68 & 0.052 & 0.318 & 0.168 & 0.052 & 0.300 & 0.160\end{array}$ $\begin{array}{llllllll}41.48 & 0.045 & 0.303 & 0.163 & 0.057 & 0.225 & 0.129\end{array}$ $\begin{array}{lllllllll}41.48 & 0.045 & 0.303 & 0.163 & 0.057 & 0.225 & 0.129\end{array}$ $\begin{array}{lllllll}42.26 & 0.032 & 0.228 & 0.153 & 0.051 & 0.194 & 0.140\end{array}$ $\begin{array}{lllllll}41.36 & 0.032 & 0.220 & 0.136 & 0.045 & 0.280 & 0.142\end{array}$ $\begin{array}{llllllll}4.84 & 0.051 & 0.266 & 0.173 & 0.052 & 0.292 & 0.133\end{array}$ $\begin{array}{llllllll}41.36 & 0.051 & 0.253 & 0.161 & 0.039 & 0.316 & 0.135\end{array}$ $\begin{array}{llllllll}42.26 & 0.050 & 0.260 & 0.166 & 0.038 & 0.320 & 0.153\end{array}$ $\begin{array}{llllllll}40.97 & 0.031 & 0.232 & 0.142 & 0.066 & 0.165 & 0.119\end{array}$ $\begin{array}{llllllll}41.68 & 0.032 & 0.234 & 0.144 & 0.070 & 0.186 & 0.126\end{array}$ $\begin{array}{lllllll}41.50 & 0.029 & 0.251 & 0.137 & 0.069 & 0.204 & 0.142\end{array}$

$\begin{array}{lllllll}41.57 & 0.043 & 0.240 & 0.146 & 0.049 & 0.223 & 0.140\end{array}$ $\begin{array}{llllllll}41.61 & 0.035 & 0.228 & 0.134 & 0.048 & 0.214 & 0.106\end{array}$ $\begin{array}{llllllll}40.70 & 0.041 & 0.245 & 0.171 & 0.052 & 0.233 & 0.150\end{array}$ $\begin{array}{llllllll}40.38 & 0.028 & 0.242 & 0.135 & 0.064 & 0.203 & 0.143\end{array}$ $\begin{array}{llllllll}41.18 & 0.030 & 0.250 & 0.135 & 0.069 & 0.178 & 0.136\end{array}$ $\begin{array}{llllllll}41.64 & 0.030 & 0.239 & 0.141 & 0.059 & 0.195 & 0.118\end{array}$ $\begin{array}{llllllll}41.61 & 0.047 & 0.241 & 0.162 & 0.058 & 0.190 & 0.106\end{array}$ $\begin{array}{llllllll}40.90 & 0.041 & 0.233 & 0.145 & 0.044 & 0.185 & 0.117\end{array}$ $\begin{array}{llllllll}41.61 & 0.042 & 0.237 & 0.157 & 0.042 & 0.186 & 0.121\end{array}$ $\begin{array}{lllllll}41.59 & 0.030 & 0.229 & 0.133 & 0.059 & 0.188 & 0.095\end{array}$ $\begin{array}{llllllll}41.82 & 0.035 & 0.227 & 0.154 & 0.069 & 0.144 & 0.099\end{array}$ $\begin{array}{lllllll}2.67 & 0.029 & 0.230 & 0.146 & 0.065 & 0.177 & 0.103\end{array}$ $\begin{array}{llllllll}41.25 & 0.034 & 0.228 & 0.150 & 0.063 & 0.147 & 0.120\end{array}$ $\begin{array}{lllllll}42.23 & 0.030 & 0.209 & 0.137 & 0.059 & 0.167 & 0.111\end{array}$ $\begin{array}{lllllll}41.59 & 0.029 & 0.226 & 0.136 & 0.055 & 0.222 & 0.135\end{array}$
100.2

100.7

99.9

100.2

99.5

99.4

100.0

100.3

99.3

101.1

100.0

99.1

100.2

100.8

100.3

100.7

100.9

100.5

100.5

99.7

99.7

100.0

100.0

99.2

99.9

99.0

100.5

98.5

100.4

100.0

98.4

97.9

99.5

99.1

99.8

99.2

100.5

99.0 
31338.00 $314 \quad 37.89$ 31538.09 $316 \quad 37.91$ 31738.16 $318 \quad 38.18$ $\begin{array}{lll}319 & 37.88\end{array}$ 31937.88 $\begin{array}{ll}320 & 38.05 \\ 321 & 38.16\end{array}$ 32138.16 $\begin{array}{ll}322 & 38.21\end{array}$ $323 \quad 38.22$ $324 \quad 38.15$ 32538.09 32638.41 $327 \quad 38.23$ $328 \quad 38.27$ $\begin{array}{ll}329 & 37.92\end{array}$ $\begin{array}{lll}330 & 38.19\end{array}$ $\begin{array}{lll}331 & 38.38\end{array}$ $\begin{array}{ll}332 & 38.22\end{array}$ $333 \quad 38.17$ 33437.96 $\begin{array}{lll}335 & 38.14\end{array}$ $336 \quad 38.22$ $337 \quad 38.17$ $338 \quad 38.13$ $\begin{array}{lll}338 & 38.13 \\ 339 & 37.47\end{array}$ $\begin{array}{lll}339 & 37.47\end{array}$ $\begin{array}{lll}340 & 37.71\end{array}$ $341 \quad 37.94$ 34238.02 $343 \quad 38.06$ 34438.21 $345 \quad 37.84$ $346 \quad 37.92$ $347 \quad 37.87$

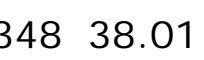
$\begin{array}{lll}349 & 37.92\end{array}$ $350 \quad 37.96$ 35137.86 $352 \quad 38.14$ $\begin{array}{lll}353 & 39.89\end{array}$ $354 \quad 37.70$ 35437.70 35539.76 35637.82 35738.07 $\begin{array}{lll}358 & 38.44\end{array}$ $\begin{array}{lll}359 & 37.77\end{array}$ $\begin{array}{llllllll}3.45 & 18.27 & 0.020 & 0.234 & 0.115 & 0.042 & 0.237 & 0.114\end{array}$ $\begin{array}{llllllll}3.36 & 18.43 & 0.020 & 0.242 & 0.117 & 0.034 & 0.209 & 0.119\end{array}$ $\begin{array}{llllllll}3.42 & 18.03 & 0.020 & 0.236 & 0.118 & 0.041 & 0.194 & 0.105\end{array}$ $\begin{array}{lllllllll}3.51 & 17.96 & 0.011 & 0.254 & 0.076 & 0.042 & 0.136 & 0.084\end{array}$ $\begin{array}{lllllllll}3.47 & 18.17 & 0.012 & 0.243 & 0.056 & 0.046 & 0.151 & 0.074\end{array}$ $\begin{array}{lllllllll}3.52 & 18.29 & 0.009 & 0.235 & 0.079 & 0.041 & 0.144 & 0.084\end{array}$ $\begin{array}{lllllllll}3.31 & 18.20 & 0.018 & 0.239 & 0.109 & 0.040 & 0.395 & 0.212\end{array}$ $\begin{array}{lllllllll}3.42 & 18.09 & 0.019 & 0.243 & 0.121 & 0.047 & 0.191 & 0.133\end{array}$ $\begin{array}{llllllll}3.53 & 18.34 & 0.019 & 0.232 & 0.098 & 0.050 & 0.176 & 0.095\end{array}$ $\begin{array}{lllllllll}3.42 & 18.42 & 0.029 & 0.233 & 0.138 & 0.043 & 0.144 & 0.089\end{array}$ $\begin{array}{lllllllll}3.41 & 18.39 & 0.027 & 0.239 & 0.120 & 0.045 & 0.166 & 0.093\end{array}$ $\begin{array}{lllllllll}3.55 & 18.12 & 0.030 & 0.267 & 0.139 & 0.044 & 0.156 & 0.082\end{array}$ $\begin{array}{lllllllll}3.64 & 18.29 & 0.040 & 0.203 & 0.147 & 0.044 & 0.159 & 0.121 \\ 3.48 & 18.06 & 0.040 & 0.204 & 0.160 & 0.046 & 0.160 & 0.133\end{array}$ $\begin{array}{llllllll}3.48 & 18.06 & 0.040 & 0.204 & 0.160 & 0.046 & 0.160 & 0.133\end{array}$ $\begin{array}{lllllllll}3.37 & 18.29 & 0.041 & 0.206 & 0.136 & 0.053 & 0.211 & 0.120\end{array}$ $\begin{array}{lllllllll}3.51 & 18.07 & 0.020 & 0.235 & 0.119 & 0.048 & 0.211 & 0.166\end{array}$ $\begin{array}{llllllll}3.46 & 18.20 & 0.021 & 0.242 & 0.121 & 0.036 & 0.315 & 0.195\end{array}$ $\begin{array}{llllllll}3.48 & 18.38 & 0.020 & 0.232 & 0.114 & 0.050 & 0.155 & 0.127\end{array}$ $\begin{array}{llllllll}3.46 & 18.37 & 0.019 & 0.215 & 0.106 & 0.036 & 0.157 & 0.093\end{array}$ $\begin{array}{llllllll}3.40 & 18.06 & 0.021 & 0.211 & 0.101 & 0.045 & 0.131 & 0.078\end{array}$ $\begin{array}{lllllllll}3.51 & 18.12 & 0.019 & 0.209 & 0.101 & 0.039 & 0.151 & 0.094 \\ 3.59 & 18.46 & 0.020 & 0.226 & 0.111 & 0.057 & 0.298 & 0.202 \\ 3.46 & 18.23 & 0.023 & 0.248 & 0.116 & 0.041 & 0.343 & 0.195\end{array}$ $\begin{array}{llllllll}3.46 & 18.23 & 0.023 & 0.248 & 0.116 & 0.041 & 0.343 & 0.195\end{array}$ $\begin{array}{lllllllll}3.48 & 18.21 & 0.021 & 0.235 & 0.108 & 0.049 & 0.303 & 0.214\end{array}$ $\begin{array}{llllllllll}3.42 & 18.25 & 0.017 & 0.235 & 0.108 & 0.049 & 0.303 & 0.214\end{array}$ $\begin{array}{llllllll}3.60 & 18.31 & 0.017 & 0.240 & 0.110 & 0.055 & 0.130 & 0.085\end{array}$ $\begin{array}{llllllll}3.36 & 18.29 & 0.018 & 0.232 & 0.108 & 0.053 & 0.149 & 0.077\end{array}$ $\begin{array}{llllllll}3.37 & 18.30 & 0.020 & 0.230 & 0.103 & 0.053 & 0.118 & 0.099\end{array}$ $\begin{array}{lllllllll}3.43 & 18.04 & 0.017 & 0.231 & 0.099 & 0.050 & 0.139 & 0.090\end{array}$ $\begin{array}{lllllllll}3.41 & 18.36 & 0.020 & 0.247 & 0.114 & 0.054 & 0.167 & 0.126\end{array}$ $\begin{array}{lllllllll}3.47 & 18.11 & 0.020 & 0.246 & 0.082 & 0.055 & 0.199 & 0.124\end{array}$ $\begin{array}{lllllllll}3.30 & 18.14 & 0.019 & 0.237 & 0.100 & 0.051 & 0.130 & 0.083\end{array}$ $\begin{array}{lllllllll}3.44 & 18.19 & 0.016 & 0.239 & 0.087 & 0.047 & 0.137 & 0.083\end{array}$ $\begin{array}{llllllll}3.50 & 18.26 & 0.017 & 0.258 & 0.128 & 0.059 & 0.113 & 0.100\end{array}$ $\begin{array}{lllllllll}3.48 & 18.20 & 0.017 & 0.244 & 0.093 & 0.052 & 0.160 & 0.084\end{array}$ $\begin{array}{llllllll}3.49 & 18.02 & 0.018 & 0.275 & 0.111 & 0.058 & 0.117 & 0.091\end{array}$ $\begin{array}{llllllll}3.35 & 17.97 & 0.015 & 0.243 & 0.100 & 0.053 & 0.127 & 0.087\end{array}$ $\begin{array}{llllllll}3.39 & 17.92 & 0.017 & 0.230 & 0.120 & 0.054 & 0.149 & 0.205\end{array}$ $\begin{array}{llllllll}3.35 & 18.17 & 0.017 & 0.250 & 0.104 & 0.049 & 0.152 & 0.098\end{array}$ $\begin{array}{lllllllll}3.65 & 18.36 & 0.019 & 0.258 & 0.128 & 0.059 & 0.327 & 0.261\end{array}$ $\begin{array}{lllllllll}3.55 & 18.92 & 0.016 & 0.227 & 0.105 & 0.052 & 0.145 & 0.084\end{array}$ $\begin{array}{lllllllll}3.42 & 17.93 & 0.017 & 0.240 & 0.120 & 0.054 & 0.148 & 0.111\end{array}$ $\begin{array}{lllllllll}3.64 & 19.10 & 0.020 & 0.249 & 0.112 & 0.047 & 0.286 & 0.174\end{array}$ $\begin{array}{llllllll}3.45 & 18.12 & 0.019 & 0.236 & 0.105 & 0.046 & 0.254 & 0.153\end{array}$ $\begin{array}{llllllll}3.39 & 18.19 & 0.020 & 0.225 & 0.109 & 0.052 & 0.242 & 0.132\end{array}$ $\begin{array}{lllllllll}3.40 & 18.23 & 0.022 & 0.236 & 0.160 & 0.056 & 0.152 & 0.137\end{array}$ $\begin{array}{lllllllll}3.42 & 18.20 & 0.016 & 0.231 & 0.103 & 0.047 & 0.144 & 0.102\end{array}$ $\begin{array}{lllllllll}3.43 & 17.86 & 0.017 & 0.247 & 0.121 & 0.054 & 0.125 & 0.097\end{array}$ $\begin{array}{llllllll}3.47 & 18.19 & 0.030 & 0.227 & 0.126 & 0.043 & 0.206 & 0.110\end{array}$
$3.05 \quad 3.36$

$53.33 \quad 3.42$

$53.07 \quad 3.51$

$53.42 \quad 3.47$

$53.03 \quad 3.31$

$53.03 \quad 3.31$

$53.42 \quad 3.53$

$53.49 \quad 3.42$

$53.51 \quad 3.41$

$53.41 \quad 3.55$

$\begin{array}{ll}53.33 & 3.64 \\ 53.77 & 3.48\end{array}$

$\begin{array}{ll}53.52 & 3.37\end{array}$

$53.58 \quad 3.51$

$53.09 \quad 3.46$

$53.47 \quad 3.48$

$53.73 \quad 3.46$

\begin{tabular}{ll}
$53.51 \quad 3.40$ \\
\hline 3.44
\end{tabular}

$\begin{array}{ll}53.14 & 3.51 \\ 53.40 & 3.46\end{array}$

$53.40 \quad 3.46$

$\begin{array}{ll}53.51 & 3.48\end{array}$

$53.44 \quad 3.42$

$53.38 \quad 3.60$

$\begin{array}{ll}52.46 & 3.36\end{array}$

$52.79 \quad 3.37$

$53.12 \quad 3.43$

$53.23 \quad 3.41$

$\begin{array}{ll}53.28 & 3.47 \\ 53.49 & 3.30\end{array}$

$52.98 \quad 3.44$

$\begin{array}{ll}52.98 & 3.44 \\ 53.09 & 3.50\end{array}$

$53.02 \quad 3.48$

$53.21 \quad 3.49$

$53.09 \quad 3.35$

$53.14 \quad 3.39$

$\begin{array}{ll}53.00 & 3.35 \\ 53.40 & 3.65\end{array}$

$55.85 \quad 3.55$

$\begin{array}{ll}55.85 & 3.55 \\ 52.78 & 3.42\end{array}$

$\begin{array}{ll}52.95 & 3.45\end{array}$

$\begin{array}{ll}53.30 & 3.39\end{array}$

$53.82 \quad 3.40$

$52.88 \quad 3.42$

$\begin{array}{ll}52.22 & 3.43 \\ 54.05 & 3.47\end{array}$ $\begin{array}{lllllll}41.87 & 0.033 & 0.234 & 0.147 & 0.054 & 0.277 & 0.145\end{array}$ $\begin{array}{llllllll}42.23 & 0.033 & 0.242 & 0.150 & 0.044 & 0.245 & 0.151\end{array}$ $\begin{array}{lllllll}41.32 & 0.033 & 0.236 & 0.152 & 0.053 & 0.227 & 0.133\end{array}$ $\begin{array}{llllllll}41.16 & 0.018 & 0.254 & 0.098 & 0.054 & 0.159 & 0.107\end{array}$ $\begin{array}{llllllll}41.64 & 0.019 & 0.243 & 0.072 & 0.059 & 0.177 & 0.094\end{array}$ $\begin{array}{llllllll}41.91 & 0.016 & 0.235 & 0.102 & 0.053 & 0.169 & 0.106\end{array}$ $\begin{array}{llllllll}41.71 & 0.030 & 0.239 & 0.140 & 0.052 & 0.463 & 0.270\end{array}$ $\begin{array}{llllllll}41.71 & 0.030 & 0.239 & 0.140 & 0.052 & 0.463 & 0.270\end{array}$ $\begin{array}{llllllll}4.45 & 0.031 & 0.243 & 0.156 & 0.060 & 0.223 & 0.169\end{array}$ $\begin{array}{lllllllll}41.45 & 0.031 & 0.243 & 0.156 & 0.060 & 0.223 & 0.169\end{array}$ $\begin{array}{llllllll}42.21 & 0.048 & 0.233 & 0.177 & 0.055 & 0.169 & 0.114\end{array}$ $\begin{array}{lllllllll}42.14 & 0.045 & 0.239 & 0.154 & 0.058 & 0.194 & 0.118\end{array}$ $\begin{array}{llllllll}41.52 & 0.049 & 0.267 & 0.179 & 0.057 & 0.183 & 0.104\end{array}$ $\begin{array}{llllllll}41.91 & 0.065 & 0.203 & 0.189 & 0.057 & 0.187 & 0.153\end{array}$ $\begin{array}{llllllll}41.39 & 0.067 & 0.204 & 0.205 & 0.059 & 0.188 & 0.169\end{array}$ $\begin{array}{llllllll}41.91 & 0.068 & 0.206 & 0.174 & 0.068 & 0.248 & 0.152\end{array}$ $\begin{array}{lllllll}41.41 & 0.033 & 0.235 & 0.154 & 0.062 & 0.247 & 0.211\end{array}$ $\begin{array}{llllllll}41.71 & 0.034 & 0.242 & 0.156 & 0.046 & 0.369 & 0.247\end{array}$ $\begin{array}{llllllll}42.12 & 0.033 & 0.232 & 0.147 & 0.064 & 0.181 & 0.161\end{array}$ $\begin{array}{llllllll}2.10 & 0.032 & 0.215 & 0.136 & 0.046 & 0.184 & 0.118\end{array}$ $\begin{array}{llllllll}41.39 & 0.035 & 0.211 & 0.129 & 0.058 & 0.154 & 0.099\end{array}$ $\begin{array}{llllllll}41.52 & 0.031 & 0.209 & 0.129 & 0.051 & 0.177 & 0.120\end{array}$ $\begin{array}{llllllll}42.30 & 0.034 & 0.226 & 0.142 & 0.074 & 0.349 & 0.256\end{array}$ $\begin{array}{llllllll}41.78 & 0.038 & 0.248 & 0.150 & 0.053 & 0.402 & 0.247\end{array}$ $\begin{array}{llllllll}41.73 & 0.035 & 0.235 & 0.139 & 0.063 & 0.354 & 0.272\end{array}$ $\begin{array}{lllllll}41.82 & 0.028 & 0.224 & 0.134 & 0.056 & 0.169 & 0.099\end{array}$ $\begin{array}{llllllll}41.96 & 0.028 & 0.240 & 0.141 & 0.071 & 0.152 & 0.108\end{array}$ $\begin{array}{llllllll}41.91 & 0.029 & 0.232 & 0.139 & 0.068 & 0.174 & 0.098\end{array}$ $\begin{array}{llllllll}4.94 & 0.033 & 0.230 & 0.133 & 0.068 & 0.138 & 0.126\end{array}$ $\begin{array}{llllllll}41.34 & 0.028 & 0.231 & 0.128 & 0.064 & 0.163 & 0.114\end{array}$ $\begin{array}{llllllll}42.07 & 0.033 & 0.247 & 0.146 & 0.070 & 0.196 & 0.161\end{array}$ $\begin{array}{llllllll}41.50 & 0.034 & 0.246 & 0.106 & 0.071 & 0.233 & 0.158\end{array}$ $\begin{array}{llllllll}41.57 & 0.032 & 0.237 & 0.129 & 0.066 & 0.152 & 0.105\end{array}$ $\begin{array}{llllllll}41.68 & 0.026 & 0.239 & 0.112 & 0.061 & 0.160 & 0.105\end{array}$ $\begin{array}{llllllll}41.84 & 0.028 & 0.258 & 0.164 & 0.077 & 0.133 & 0.128\end{array}$ $\begin{array}{llllllll}41.71 & 0.028 & 0.244 & 0.119 & 0.067 & 0.188 & 0.107\end{array}$ $\begin{array}{llllllll}41.29 & 0.030 & 0.275 & 0.143 & 0.075 & 0.137 & 0.116\end{array}$ $\begin{array}{llllllll}4.18 & 0.025 & 0.243 & 0.128 & 0.069 & 0.148 & 0.110\end{array}$ $\begin{array}{llllllll}41.06 & 0.027 & 0.230 & 0.154 & 0.070 & 0.174 & 0.260\end{array}$ $\begin{array}{llllllll}41.64 & 0.027 & 0.250 & 0.134 & 0.063 & 0.178 & 0.124\end{array}$ $\begin{array}{llllllll}42.07 & 0.032 & 0.258 & 0.164 & 0.076 & 0.383 & 0.331\end{array}$ $\begin{array}{llllllll}43.36 & 0.026 & 0.227 & 0.135 & 0.068 & 0.169 & 0.107\end{array}$ $\begin{array}{llllllll}41.09 & 0.028 & 0.240 & 0.154 & 0.070 & 0.173 & 0.140\end{array}$ $\begin{array}{lllllll}43.77 & 0.034 & 0.249 & 0.144 & 0.061 & 0.335 & 0.221\end{array}$ $\begin{array}{llllllll}41.52 & 0.031 & 0.236 & 0.135 & 0.059 & 0.298 & 0.195\end{array}$ $\begin{array}{llllllll}41.68 & 0.033 & 0.225 & 0.140 & 0.067 & 0.283 & 0.168\end{array}$ $\begin{array}{llllllll}41.78 & 0.036 & 0.236 & 0.206 & 0.072 & 0.178 & 0.173\end{array}$ $\begin{array}{llllllll}41.71 & 0.026 & 0.231 & 0.133 & 0.061 & 0.168 & 0.129\end{array}$ $\begin{array}{llllllll}40.93 & 0.028 & 0.247 & 0.155 & 0.070 & 0.147 & 0.123\end{array}$ $\begin{array}{lllllll}41.68 & 0.050 & 0.227 & 0.162 & 0.056 & 0.241 & 0.139\end{array}$
99.5

98.9

99.8

99.9

99.7

99.5

99.7

99.3

100.0

99.2

100.1

99.8

99.8

99.4

99.7

98.8

98.6

99.6

99.1

98.8

99.0

98.8

98.3

00.4

103.5

104.1

98.9

99.3

98.8

97.3

100.1 
$362 \quad 39.14$ $\begin{array}{lll}363 & 38.17\end{array}$ $\begin{array}{lll}364 & 38.27\end{array}$ $\begin{array}{ll}365 & 39.24\end{array}$ $366 \quad 38.34$ $367 \quad 38.39$ $\begin{array}{lll}368 & 38.31 \\ 369 & 38.22\end{array}$ $\begin{array}{ll}369 & 38.22\end{array}$ $370 \quad 38.02$ $371 \quad 37.64$

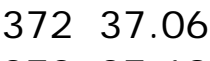
$373 \quad 37.19$ $\begin{array}{lll}374 & 38.38\end{array}$ $\begin{array}{ll}375 & 38.13\end{array}$ $\begin{array}{ll}376 & 38.34 \\ 377 & 37.76\end{array}$ $\begin{array}{lll}377 & 37.76\end{array}$ $\begin{array}{lll}378 & 37.90\end{array}$ $\begin{array}{lll}379 & 37.85\end{array}$ $\begin{array}{ll}380 & 38.10 \\ 381 & 38.49\end{array}$ 38138.49 38239.02 $383 \quad 38.60$ $384 \quad 37.62$ $\begin{array}{lll}385 & 37.96\end{array}$ $386 \quad 37.90$ $\begin{array}{ll}387 & 37.63\end{array}$ $\begin{array}{lll}388 & 37.92\end{array}$ $389 \quad 38.04$ 39038.23 Hagan, 39238.03 $393 \quad 38.31$ 39438.18 $\begin{array}{lll}394 & 38.18\end{array}$ $\begin{array}{ll}395 & 38.37\end{array}$ $\begin{array}{lll}396 & 38.13\end{array}$ $\begin{array}{lll}397 & 38.00\end{array}$ $398 \quad 37.83$ $399 \quad 38.05$ $400 \quad 38.25$ $401 \quad 38.17$ 40238.26 40238.26 40338.53 40438.18 $\begin{array}{lll}05 & 38.23\end{array}$ $406 \quad 38.21$ $407 \quad 38.19$ $408 \quad 38.59$ 40938.65 $\begin{array}{llllllll}3.58 & 18.40 & 0.033 & 0.251 & 0.132 & 0.035 & 0.174 & 0.106\end{array}$ $\begin{array}{llllllll}3.44 & 18.24 & 0.031 & 0.234 & 0.119 & 0.034 & 0.233 & 0.131\end{array}$ $\begin{array}{lllllllll}3.38 & 18.19 & 0.016 & 0.243 & 0.111 & 0.055 & 0.169 & 0.137\end{array}$ $\begin{array}{lllllllll}3.50 & 18.87 & 0.015 & 0.232 & 0.095 & 0.054 & 0.245 & 0.170\end{array}$ $\begin{array}{llllllll}3.52 & 18.15 & 0.016 & 0.259 & 0.101 & 0.060 & 0.252 & 0.191\end{array}$ $\begin{array}{lllllllll}3.52 & 17.76 & 0.016 & 0.237 & 0.100 & 0.050 & 0.138 & 0.101\end{array}$ $\begin{array}{llllllll}3.38 & 18.37 & 0.019 & 0.248 & 0.118 & 0.052 & 0.118 & 0.092\end{array}$ $\begin{array}{llllllll}3.46 & 18.20 & 0.018 & 0.246 & 0.082 & 0.058 & 0.125 & 0.094\end{array}$ $\begin{array}{llllllll}3.41 & 18.01 & 0.025 & 0.229 & 0.120 & 0.047 & 0.193 & 0.147\end{array}$ $\begin{array}{lllllllll}3.34 & 17.85 & 0.023 & 0.247 & 0.105 & 0.047 & 0.195 & 0.127\end{array}$ $\begin{array}{lllllllll}3.19 & 17.41 & 0.022 & 0.234 & 0.109 & 0.049 & 0.196 & 0.138\end{array}$ $\begin{array}{lllllllll}3.45 & 17.89 & 0.023 & 0.233 & 0.117 & 0.038 & 0.163 & 0.087\end{array}$ $\begin{array}{lllllllll}3.62 & 18.26 & 0.025 & 0.232 & 0.130 & 0.035 & 0.158 & 0.097\end{array}$ $\begin{array}{llllllll}3.33 & 17.90 & 0.026 & 0.245 & 0.108 & 0.036 & 0.155 & 0.084\end{array}$ $\begin{array}{llllllll}3.40 & 18.10 & 0.019 & 0.252 & 0.102 & 0.050 & 0.147 & 0.107\end{array}$ $\begin{array}{llllllll}3.39 & 18.03 & 0.016 & 0.227 & 0.103 & 0.043 & 0.185 & 0.113\end{array}$ $\begin{array}{lllllllll}3.43 & 17.93 & 0.016 & 0.234 & 0.113 & 0.060 & 0.170 & 0.132\end{array}$ $\begin{array}{lllllllll}3.42 & 18.19 & 0.015 & 0.230 & 0.109 & 0.053 & 0.180 & 0.121\end{array}$ $\begin{array}{lllllllll}3.44 & 18.45 & 0.018 & 0.223 & 0.103 & 0.057 & 0.182 & 0.134\end{array}$ $\begin{array}{llllllll}3.40 & 18.54 & 0.015 & 0.250 & 0.105 & 0.051 & 0.193 & 0.120\end{array}$ $\begin{array}{lllllllll}3.16 & 17.96 & 0.020 & 0.239 & 0.134 & 0.059 & 0.128 & 0.079\end{array}$ $\begin{array}{lllllllll}3.28 & 18.66 & 0.017 & 0.229 & 0.105 & 0.047 & 0.133 & 0.079\end{array}$ $\begin{array}{llllllll}3.39 & 18.05 & 0.019 & 0.232 & 0.113 & 0.053 & 0.134 & 0.093\end{array}$ $\begin{array}{lllllllll}3.40 & 18.20 & 0.018 & 0.226 & 0.106 & 0.042 & 0.136 & 0.067\end{array}$ $\begin{array}{llllllll}3.38 & 17.90 & 0.022 & 0.229 & 0.121 & 0.041 & 0.110 & 0.072\end{array}$ $\begin{array}{lllllllll}3.28 & 18.10 & 0.020 & 0.214 & 0.097 & 0.042 & 0.165 & 0.073\end{array}$ $\begin{array}{llllllll}3.57 & 18.03 & 0.011 & 0.243 & 0.082 & 0.073 & 0.107 & 0.104\end{array}$ $\begin{array}{lllllllll}3.52 & 18.11 & 0.008 & 0.229 & 0.060 & 0.069 & 0.146 & 0.111\end{array}$ $\begin{array}{llllllll}3.47 & 18.61 & 0.013 & 0.233 & 0.087 & 0.082 & 0.092 & 0.100\end{array}$ nia Millbrig \#5

$\begin{array}{llllllll}3.52 & 18.37 & 0.075 & 0.189 & 0.176 & 0.039 & 0.102 & 0.073\end{array}$ $\begin{array}{lllllllll}3.45 & 18.39 & 0.079 & 0.177 & 0.186 & 0.037 & 0.102 & 0.068\end{array}$ $\begin{array}{llllllll}3.45 & 18.39 & 0.079 & 0.177 & 0.186 & 0.037 & 0.102 & 0.068 \\ 3.56 & 18.09 & 0.075 & 0.178 & 0.167 & 0.037 & 0.118 & 0.076\end{array}$ $\begin{array}{lllllllll}3.36 & 18.13 & 0.087 & 0.194 & 0.237 & 0.059 & 0.140 & 0.113\end{array}$ $\begin{array}{llllllll}3.63 & 18.20 & 0.082 & 0.202 & 0.208 & 0.047 & 0.158 & 0.100\end{array}$ $\begin{array}{llllllll}3.43 & 17.91 & 0.083 & 0.203 & 0.223 & 0.055 & 0.129 & 0.106\end{array}$ $\begin{array}{lllllllll}3.42 & 17.98 & 0.010 & 0.245 & 0.072 & 0.061 & 0.192 & 0.119\end{array}$ $\begin{array}{lllllllll}3.49 & 18.08 & 0.013 & 0.232 & 0.076 & 0.074 & 0.118 & 0.089\end{array}$ $\begin{array}{lllllllll}3.58 & 18.43 & 0.010 & 0.227 & 0.073 & 0.068 & 0.125 & 0.090\end{array}$ $\begin{array}{llllllll}3.34 & 18.32 & 0.009 & 0.244 & 0.086 & 0.073 & 0.120 & 0.134\end{array}$ $\begin{array}{lllllllll}3.52 & 18.14 & 0.011 & 0.233 & 0.082 & 0.074 & 0.108 & 0.123\end{array}$ $\begin{array}{lllllllll}3.52 & 18.14 & 0.011 & 0.233 & 0.082 & 0.074 & 0.108 & 0.123\end{array}$ $\begin{array}{llllllll}3.30 & 18.26 & 0.018 & 0.251 & 0.109 & 0.053 & 0.126 & 0.075\end{array}$ $\begin{array}{lllllllll}3.46 & 18.24 & 0.018 & 0.230 & 0.104 & 0.048 & 0.133 & 0.082\end{array}$ $\begin{array}{llllllll}3.49 & 18.12 & 0.017 & 0.226 & 0.108 & 0.043 & 0.126 & 0.088\end{array}$ $\begin{array}{lllllllll}3.42 & 18.31 & 0.010 & 0.234 & 0.063 & 0.076 & 0.105 & 0.133\end{array}$ $\begin{array}{llllllll}3.38 & 17.87 & 0.064 & 0.175 & 0.098 & 0.051 & 0.147 & 0.170\end{array}$ $\begin{array}{lllllllll}3.41 & 18.08 & 0.010 & 0.229 & 0.072 & 0.072 & 0.140 & 0.172\end{array}$ $\begin{array}{lllllllll}3.72 & 18.12 & 0.008 & 0.223 & 0.070 & 0.086 & 0.127 & 0.142\end{array}$
$54.80 \quad 3.58$

$3.44 \quad 3.44$

3.38

$54.94 \quad 3.50$

$53.68 \quad 3.52$

$3.75 \quad 3.52$

$53.63 \quad 3.38$

$3.51 \quad 3.46$

$5.23 \quad 3.41$

$52.70 \quad 3.34$

$51.88 \quad 3.19$

$\begin{array}{ll}52.07 & 3.45 \\ 53.73 & 3.62\end{array}$

$\begin{array}{ll}53.73 & 3.62 \\ 53.38 & 3.33\end{array}$

$53.68 \quad 3.40$

$52.86 \quad 3.39$

53.06

52.99

53.34

53.89

54.63

54.04

52.67

53.14

53.06

52.68

53.09
53.26
53.52 $\begin{array}{llllllll}42.16 & 0.054 & 0.251 & 0.169 & 0.046 & 0.204 & 0.134\end{array}$ $\begin{array}{lllllll}41.80 & 0.052 & 0.234 & 0.154 & 0.044 & 0.273 & 0.166\end{array}$ $\begin{array}{llllllll}41.68 & 0.026 & 0.243 & 0.143 & 0.071 & 0.198 & 0.174\end{array}$ $\begin{array}{llllllll}43.24 & 0.025 & 0.232 & 0.122 & 0.069 & 0.287 & 0.215\end{array}$ $\begin{array}{llllllll}41.59 & 0.027 & 0.259 & 0.130 & 0.078 & 0.295 & 0.242\end{array}$ $\begin{array}{llllllll}40.70 & 0.026 & 0.237 & 0.129 & 0.064 & 0.161 & 0.128\end{array}$ $\begin{array}{llllllll}42.10 & 0.032 & 0.248 & 0.152 & 0.068 & 0.138 & 0.117\end{array}$ $\begin{array}{lllllll}41.71 & 0.029 & 0.246 & 0.105 & 0.075 & 0.146 & 0.119\end{array}$ $\begin{array}{llllllll}41.27 & 0.041 & 0.229 & 0.154 & 0.061 & 0.226 & 0.186\end{array}$ $\begin{array}{llllllll}40.90 & 0.038 & 0.247 & 0.135 & 0.060 & 0.228 & 0.161\end{array}$ $\begin{array}{llllllll}39.90 & 0.037 & 0.234 & 0.140 & 0.064 & 0.230 & 0.176\end{array}$ $\begin{array}{llllllll}41.00 & 0.038 & 0.233 & 0.151 & 0.049 & 0.190 & 0.110\end{array}$ $\begin{array}{llllllll}41.84 & 0.041 & 0.232 & 0.167 & 0.046 & 0.186 & 0.123\end{array}$ $\begin{array}{llllllll}41.02 & 0.043 & 0.245 & 0.139 & 0.046 & 0.181 & 0.107\end{array}$ $\begin{array}{llllllll}41.48 & 0.031 & 0.252 & 0.131 & 0.064 & 0.172 & 0.136\end{array}$ $\begin{array}{lllll}41.32 & 0.026 & 0.227 & 0.132\end{array}$ $\begin{array}{lllll}0.027 & 0.234 & 0.132 & 0.05\end{array}$ $\begin{array}{llllllll}41.09 & 0.027 & 0.234 & 0.146 & 0.078 & 0.199 & 0.167\end{array}$ $\begin{array}{llllllllll}41.68 & 0.027 & 0.234 & 0.146 & 0.078 & 0.199 & 0.167\end{array}$ $\begin{array}{lllllllll}4.28 & 0.029 & 0.223 & 0.132 & 0.073 & 0.213 & 0.170\end{array}$ $\begin{array}{llllllll}42.49 & 0.025 & 0.250 & 0.134 & 0.066 & 0.226 & 0.152\end{array}$ $\begin{array}{llllllll}41.16 & 0.033 & 0.239 & 0.172 & 0.076 & 0.149 & 0.101\end{array}$ $\begin{array}{llllllll}42.76 & 0.029 & 0.229 & 0.136 & 0.061 & 0.156 & 0.101\end{array}$ $\begin{array}{llllllll}41.36 & 0.031 & 0.232 & 0.146 & 0.069 & 0.157 & 0.118\end{array}$ $\begin{array}{llllllll}41.71 & 0.030 & 0.226 & 0.136 & 0.055 & 0.159 & 0.085\end{array}$ $\begin{array}{llllllll}41.71 & 0.030 & 0.226 & 0.136 & 0.055 & 0.159 & 0.085\end{array}$ $\begin{array}{lllllllll}41.02 & 0.036 & 0.229 & 0.155 & 0.053 & 0.129 & 0.091\end{array}$ $\begin{array}{llllllll}41.48 & 0.034 & 0.214 & 0.124 & 0.054 & 0.193 & 0.092\end{array}$ $\begin{array}{llllllll}41.32 & 0.019 & 0.243 & 0.106 & 0.094 & 0.125 & 0.132 \\ 41.50 & 0.013 & 0.229 & 0.077 & 0.089 & 0.170 & 0.141\end{array}$ $\begin{array}{llllllll}42.65 & 0.021 & 0.233 & 0.112 & 0.106 & 0.108 & 0.128\end{array}$

$\begin{array}{lllllll}42.10 & 0.124 & 0.189 & 0.226 & 0.050 & 0.119 & 0.093\end{array}$ $\begin{array}{llllllll}42.14 & 0.131 & 0.177 & 0.240 & 0.048 & 0.120 & 0.086\end{array}$ $\begin{array}{llllllll}41.45 & 0.124 & 0.178 & 0.215 & 0.048 & 0.138 & 0.096\end{array}$ $\begin{array}{lllllll}41.55 & 0.145 & 0.194 & 0.305 & 0.076 & 0.164 & 0.143\end{array}$ $\begin{array}{llllllll}41.71 & 0.135 & 0.202 & 0.268 & 0.060 & 0.185 & 0.127\end{array}$ $\begin{array}{llllllll}41.04 & 0.138 & 0.203 & 0.287 & 0.071 & 0.151 & 0.134\end{array}$ $\begin{array}{llllllll}41.20 & 0.016 & 0.245 & 0.093 & 0.079 & 0.225 & 0.151\end{array}$ $\begin{array}{llllllll}41.43 & 0.021 & 0.232 & 0.097 & 0.095 & 0.138 & 0.113\end{array}$ $\begin{array}{llllllll}42.23 & 0.016 & 0.227 & 0.094 & 0.088 & 0.146 & 0.114\end{array}$ $\begin{array}{llllllll}41.98 & 0.015 & 0.244 & 0.111 & 0.094 & 0.140 & 0.170\end{array}$ $\begin{array}{llllllll}41.57 & 0.018 & 0.233 & 0.106 & 0.096 & 0.126 & 0.156\end{array}$ $\begin{array}{llllllll}41.61 & 0.017 & 0.225 & 0.104 & 0.082 & 0.153 & 0.160\end{array}$ $\begin{array}{lllllllll}41.61 & 0.017 & 0.225 & 0.104 & 0.082 & 0.153 & 0.160\end{array}$ $\begin{array}{lllllllll}41.84 & 0.030 & 0.251 & 0.140 & 0.068 & 0.147 & 0.096\end{array}$ $\begin{array}{lllllllll}41.80 & 0.029 & 0.230 & 0.134 & 0.062 & 0.155 & 0.104\end{array}$ $\begin{array}{llllllll}41.52 & 0.028 & 0.226 & 0.139 & 0.056 & 0.147 & 0.112\end{array}$ $\begin{array}{lllllll}41.96 & 0.017 & 0.234 & 0.081 & 0.098 & 0.123 & 0.169\end{array}$ $\begin{array}{lllllll}40.95 & 0.106 & 0.175 & 0.125 & 0.066 & 0.173 & 0.216\end{array}$ $\begin{array}{lllllll}41.43 & 0.017 & 0.229 & 0.093 & 0.093 & 0.164 & 0.219\end{array}$ $\begin{array}{lllllll}41.52 & 0.014 & 0.223 & 0.090 & 0.111 & 0.148 & 0.180\end{array}$
101.4

99.6

99.5

102.

99.8

98.7

99.9

99.4

97.8

95.8

97.3

100.0

98.5

99.3

98.9

99.9

100.6

99.7

100.8

98.2

98.2

99.0

100.3

99.8

99.6

99.

99.4

00.0

98.6

98.6

99.8

99.6

99.5

99.4

99.2

99.6
98.7

99.7

100.1 
41038.14 $411 \quad 38.48$ $412 \quad 38.24$ $413 \quad 38.53$ $414 \quad 38.72$ $415 \quad 38.51$ $416 \quad 38.62$ 41638.62 41738.10 $\begin{array}{lll}18 & 38.24\end{array}$ $419 \quad 38.27$ $420 \quad 38.59$ $421 \quad 38.35$ $422 \quad 38.76$ $423 \quad 37.47$ $424 \quad 35.04$ 42435.04 42543.14 42638.30 $\begin{array}{lll}427 & 38.12\end{array}$ $\begin{array}{lll}428 & 38.14\end{array}$ $\begin{array}{lll}429 & 38.37\end{array}$ $430 \quad 37.86$ $431 \quad 38.49$ $432 \quad 38.36$ $433 \quad 38.44$ 43438.60 $435 \quad 38.10$ $436 \quad 38.72$ $437 \quad 38.35$ $438 \quad 38.84$ $439 \quad 38.35$ $440 \quad 38.62$ $441 \quad 38.84$ $442 \quad 38.25$ $443 \quad 38.48$ $444 \quad 38.62$ $445 \quad 38.58$ $446 \quad 38.60$ $447 \quad 38.65$ $448 \quad 38.28$ $449 \quad 38.70$ $450 \quad 38.93$ 45138.60 45138.60 45238.66 45338.41 $\begin{array}{lll}454 & 38.34\end{array}$ $\begin{array}{lll}455 & 38.24\end{array}$ $456 \quad 38.11$ $\begin{array}{llllllll}3.58 & 18.05 & 0.012 & 0.207 & 0.076 & 0.080 & 0.112 & 0.140\end{array}$ $\begin{array}{lllllllll}3.49 & 18.32 & 0.007 & 0.214 & 0.055 & 0.079 & 0.088 & 0.140\end{array}$ $\begin{array}{lllllllll}3.61 & 18.09 & 0.008 & 0.186 & 0.075 & 0.049 & 0.095 & 0.124\end{array}$ $\begin{array}{llllllll}3.57 & 18.25 & 0.009 & 0.186 & 0.068 & 0.081 & 0.076 & 0.116\end{array}$ $\begin{array}{lllllllll}3.44 & 18.67 & 0.007 & 0.180 & 0.073 & 0.089 & 0.103 & 0.141\end{array}$ $\begin{array}{llllllll}3.59 & 18.31 & 0.081 & 0.316 & 0.201 & 0.046 & 0.127 & 0.087\end{array}$ $\begin{array}{lllllllll}3.34 & 18.34 & 0.081 & 0.181 & 0.203 & 0.046 & 0.107 & 0.068\end{array}$ $\begin{array}{llllllll}3.43 & 17.96 & 0.076 & 0.191 & 0.198 & 0.042 & 0.131 & 0.094\end{array}$ $\begin{array}{lllllllll}3.43 & 18.10 & 0.075 & 0.198 & 0.200 & 0.048 & 0.184 & 0.151\end{array}$ $\begin{array}{lllllllll}3.44 & 18.09 & 0.080 & 0.187 & 0.181 & 0.043 & 0.179 & 0.138\end{array}$ $\begin{array}{lllllllll}3.39 & 18.38 & 0.076 & 0.178 & 0.212 & 0.056 & 0.132 & 0.103\end{array}$ $\begin{array}{lllllllll}3.52 & 18.27 & 0.009 & 0.226 & 0.064 & 0.066 & 0.098 & 0.091\end{array}$ $\begin{array}{lllllllll}3.54 & 18.35 & 0.009 & 0.233 & 0.065 & 0.069 & 0.080 & 0.095\end{array}$ $\begin{array}{lllllllll}3.48 & 17.83 & 0.009 & 0.217 & 0.067 & 0.066 & 0.099 & 0.100\end{array}$ $\begin{array}{lllllllll}2.96 & 16.26 & 0.075 & 0.181 & 0.208 & 0.044 & 0.117 & 0.109\end{array}$ $\begin{array}{lllllllll}3.51 & 18.16 & 0.078 & 0.183 & 0.199 & 0.042 & 0.137 & 0.103\end{array}$ $\begin{array}{lllllllll}3.56 & 18.41 & 0.082 & 0.183 & 0.203 & 0.049 & 0.130 & 0.120\end{array}$ $\begin{array}{llllllll}3.66 & 18.34 & 0.077 & 0.170 & 0.218 & 0.041 & 0.158 & 0.122\end{array}$ $\begin{array}{lllllllll}3.51 & 18.08 & 0.077 & 0.188 & 0.227 & 0.049 & 0.138 & 0.117\end{array}$ $\begin{array}{llllllll}3.56 & 18.13 & 0.074 & 0.186 & 0.192 & 0.059 & 0.166 & 0.120\end{array}$ $\begin{array}{lllllllll}3.44 & 17.81 & 0.075 & 0.223 & 0.232 & 0.053 & 0.247 & 0.202\end{array}$ $\begin{array}{lllllllll}3.48 & 18.44 & 0.073 & 0.189 & 0.220 & 0.055 & 0.236 & 0.184\end{array}$ $\begin{array}{lllllllll}3.45 & 18.46 & 0.077 & 0.202 & 0.233 & 0.055 & 0.239 & 0.200\end{array}$ $\begin{array}{lllllllll}3.53 & 18.26 & 0.067 & 0.195 & 0.211 & 0.048 & 0.190 & 0.123\end{array}$ $\begin{array}{lllllllll}3.51 & 18.26 & 0.068 & 0.204 & 0.215 & 0.063 & 0.164 & 0.133\end{array}$ $\begin{array}{lllllllll}3.43 & 18.19 & 0.068 & 0.201 & 0.201 & 0.057 & 0.187 & 0.134\end{array}$ $\begin{array}{llllllll}3.44 & 18.23 & 0.017 & 0.243 & 0.133 & 0.053 & 0.129 & 0.090\end{array}$ $\begin{array}{llllllll}3.46 & 18.20 & 0.016 & 0.228 & 0.105 & 0.049 & 0.138 & 0.093\end{array}$ $\begin{array}{lllllllll}3.41 & 18.71 & 0.019 & 0.244 & 0.113 & 0.054 & 0.088 & 0.081\end{array}$ $\begin{array}{lllllllll}3.45 & 18.21 & 0.065 & 0.179 & 0.155 & 0.037 & 0.095 & 0.075\end{array}$ $\begin{array}{lllllllll}3.68 & 18.18 & 0.070 & 0.177 & 0.169 & 0.033 & 0.100 & 0.077\end{array}$ $\begin{array}{lllllllll}3.52 & 18.17 & 0.067 & 0.177 & 0.157 & 0.033 & 0.127 & 0.079\end{array}$ $\begin{array}{lllllllll}3.45 & 18.03 & 0.024 & 0.178 & 0.131 & 0.038 & 0.108 & 0.086\end{array}$ $\begin{array}{llllllll}3.43 & 18.35 & 0.024 & 0.187 & 0.125 & 0.043 & 0.148 & 0.092\end{array}$ $\begin{array}{lllllllll}3.51 & 18.37 & 0.024 & 0.163 & 0.128 & 0.043 & 0.115 & 0.083\end{array}$ $\begin{array}{llllllll}3.42 & 18.11 & 0.068 & 0.171 & 0.154 & 0.038 & 0.094 & 0.067\end{array}$ $\begin{array}{llllllll}3.67 & 18.32 & 0.069 & 0.189 & 0.166 & 0.043 & 0.084 & 0.074\end{array}$ $\begin{array}{lllllllll}3.45 & 18.15 & 0.076 & 0.174 & 0.160 & 0.036 & 0.115 & 0.074\end{array}$ $\begin{array}{lllllllll}3.57 & 18.33 & 0.051 & 0.211 & 0.181 & 0.046 & 0.241 & 0.164\end{array}$ $\begin{array}{lllllllll}3.45 & 18.37 & 0.050 & 0.200 & 0.171 & 0.042 & 0.235 & 0.155\end{array}$ $\begin{array}{llllllll}3.36 & 18.51 & 0.050 & 0.199 & 0.178 & 0.042 & 0.212 & 0.136\end{array}$ $\begin{array}{lllllllll}3.58 & 18.38 & 0.009 & 0.224 & 0.064 & 0.065 & 0.106 & 0.088\end{array}$ $\begin{array}{llllllll}3.57 & 18.35 & 0.008 & 0.241 & 0.071 & 0.069 & 0.104 & 0.100\end{array}$ $\begin{array}{llllllll}3.59 & 18.47 & 0.009 & 0.223 & 0.056 & 0.066 & 0.113 & 0.095\end{array}$ $\begin{array}{lllllllll}3.45 & 18.42 & 0.018 & 0.230 & 0.113 & 0.054 & 0.158 & 0.099\end{array}$ $\begin{array}{lllllllll}3.61 & 18.30 & 0.016 & 0.235 & 0.101 & 0.049 & 0.147 & 0.081\end{array}$ $\begin{array}{llllllll}3.41 & 17.98 & 0.019 & 0.238 & 0.120 & 0.052 & 0.144 & 0.089\end{array}$ $\begin{array}{llllllll}3.34 & 18.05 & 0.006 & 0.224 & 0.059 & 0.082 & 0.106 & 0.125\end{array}$ $\begin{array}{llllllll}3.64 & 18.24 & 0.007 & 0.157 & 0.038 & 0.048 & 0.080 & 0.145\end{array}$
$53.40 \quad 3.58$

$53.87 \quad 3.49$

3.61

$53.94 \quad 3.57$

$54.21 \quad 3.44$

$53.91 \quad 3.59$

$54.07 \quad 3.34$

$53.34 \quad 3.43$

$\begin{array}{ll}53.54 & 3.43\end{array}$

$53.58 \quad 3.44$

$\begin{array}{ll}54.03 & 3.39\end{array}$

$53.69 \quad 3.52$

$54.26 \quad 3.54$

$49.06 \quad 2.96$

$60.40 \quad 3.51$

$53.62 \quad 3.56$

$53.37 \quad 3.66$

$53.40 \quad 3.51$

$53.72 \quad 3.56$

$53.00 \quad 3.44$

$\begin{array}{ll}53.89 & 3.48 \\ 53.70 & 3.45 \\ 53.82 & 3.53\end{array}$

$53.82 \quad 3.53$

$54.04 \quad 3.51$

$53.34 \quad 3.43$

$\begin{array}{ll}54.21 & 3.44\end{array}$

$53.69 \quad 3.46$

54.38

53.69

54.07

54.38

53.55

53.87

54.07

54.01
54.04

54.04
54.11

54.11

54.18

54.50

54.04

54.12

53.7

53.68
53.54

53.54
53.35

53.58

54.63 $\begin{array}{lllll}41.36 & 0.020 & 0.207 & 0.098\end{array}$

$\begin{array}{lllll}41.98 & 0.011 & 0.214 & 0.071\end{array}$

$\begin{array}{lllll}41.45 & 0.014 & 0.186 & 0.097\end{array}$

$\begin{array}{llll}41.82 & 0.014 & 0.186 & 0.088 \\ 42.78 & 0.012 & 0.180 & 0.094\end{array}$

$\begin{array}{lllll}41.96 & 0.135 & 0.316 & 0.258\end{array}$

$\begin{array}{lllll}42.03 & 0.134 & 0.181 & 0.261\end{array}$

$\begin{array}{lllll}41.16 & 0.127 & 0.191 & 0.255\end{array}$

$\begin{array}{llll}41.48 & 0.125 & 0.198 & 0.257\end{array}$

$\begin{array}{lllll}41.45 & 0.132 & 0.187 & 0.233\end{array}$

$\begin{array}{lllll}42.12 & 0.127 & 0.178 & 0.272\end{array}$

$\begin{array}{lllll}41.87 & 0.014 & 0.226 & 0.082\end{array}$

$\begin{array}{lllll}42.05 & 0.015 & 0.233 & 0.084\end{array}$

$\begin{array}{lllll}40.86 & 0.014 & 0.217 & 0.086\end{array}$

$\begin{array}{lllll}37.26 & 0.124 & 0.181 & 0.268\end{array}$

$\begin{array}{lllll}41.61 & 0.129 & 0.183 & 0.255\end{array}$

$\begin{array}{llll}42.19 & 0.135 & 0.183 & 0.261\end{array}$

$\begin{array}{llll}42.03 & 0.127 & 0.170 & 0.281\end{array}$

$\begin{array}{lllll}4.43 & 0.128 & 0.188 & 0.292\end{array}$

$\begin{array}{lllll}41.55 & 0.122 & 0.186 & 0.247\end{array}$

$\begin{array}{lllll}40.81 & 0.125 & 0.223 & 0.298\end{array}$

$\begin{array}{lllll}42.26 & 0.121 & 0.189 & 0.283\end{array}$

$\begin{array}{lllll}42.30 & 0.127 & 0.202 & 0.299\end{array}$

$\begin{array}{lllll}41.84 & 0.111 & 0.195 & 0.271\end{array}$

$\begin{array}{lllll}41.84 & 0.113 & 0.204 & 0.276\end{array}$

$\begin{array}{lllll}41.68 & 0.113 & 0.201 & 0.259\end{array}$

41.78

41.71

42.88

41.73

41.66

41.64

41.32

42.05
42.10

42.10

41.98

41.5

42.00

42.10

42.42

42.05

42.33

42.21

41.94

41.20

$\begin{array}{lll}0.113 & 0.201 & 0.259\end{array}$

$\begin{array}{lll}0.029 & 0.243 & 0.171 \\ 0.026 & 0.228 & 0.135\end{array}$

$\begin{array}{lll}0.031 & 0.244 & 0.145\end{array}$

$\begin{array}{lll}0.107 & 0.179 & 0.199\end{array}$

$\begin{array}{lll}0.116 & 0.177 & 0.218 \\ 0.112 & 0.177 & 0.202\end{array}$

$\begin{array}{llll}0.039 & 0.178 & 0.169\end{array}$

$\begin{array}{lll}0.040 & 0.187 & 0.161\end{array}$

$\begin{array}{lll}0.040 & 0.163 & 0.164\end{array}$

$\begin{array}{lll}0.113 & 0.171 & 0.198\end{array}$

$\begin{array}{lll}0.125 & 0.174 & 0.206\end{array}$

$\begin{array}{lll}084 & 0.211 & 0.232\end{array}$

$\begin{array}{lll}0.083 & 0.200 & 0.220\end{array}$

$\begin{array}{llll}0.016 & 0.224 & 0.082\end{array}$

$\begin{array}{lll}0.013 & 0.241 & 0.091\end{array}$

$\begin{array}{lll}0.015 & 0.223 & 0.072\end{array}$

$\begin{array}{lll}0.031 & 0.230 & 0.145\end{array}$

$\begin{array}{lll}0.026 & 0.235 & 0.130\end{array}$

$\begin{array}{lll}0.032 & 0.238 & 0.155 \\ 0.011 & 0.224 & 0.075\end{array}$

$\begin{array}{lllll}41.80 & 0.011 & 0.157 & 0.049\end{array}$ $\begin{array}{lll}0.104 & 0.132 & 0.178\end{array}$

$\begin{array}{llll}0.102 & 0.103 & 0.177\end{array}$

$\begin{array}{lll}0.063 & 0.111 & 0.157\end{array}$

$\begin{array}{lll}105 & 0.089 & 0.147\end{array}$

$\begin{array}{lll}0.115 & 0.121 & 0.178\end{array}$

$\begin{array}{lll}0.060 & 0.149 & 0.110\end{array}$

$\begin{array}{lll}0.059 & 0.126 & 0.086\end{array}$

$\begin{array}{llll}0.054 & 0.153 & 0.119\end{array}$

$\begin{array}{lll}0.062 & 0.215 & 0.192\end{array}$

$\begin{array}{lll}0.056 & 0.210 & 0.175\end{array}$

$\begin{array}{lll}0.073 & 0.155 & 0.131\end{array}$

$\begin{array}{lll}0.085 & 0.114 & 0.116\end{array}$

$\begin{array}{lll}089 & 0.093 & 0.121\end{array}$

$\begin{array}{llll}0.085 & 0.116 & 0.126\end{array}$

$\begin{array}{llll}0.057 & 0.137 & 0.139\end{array}$

$\begin{array}{llll}0.054 & 0.160 & 0.131\end{array}$

$\begin{array}{llll}0.063 & 0.153 & 0.152\end{array}$

$\begin{array}{llll}0.052 & 0.184 & 0.155\end{array}$

$\begin{array}{llll}0.063 & 0.162 & 0.148\end{array}$

$\begin{array}{llll}0.077 & 0.194 & 0.152\end{array}$

$\begin{array}{llll}0.069 & 0.289 & 0.257\end{array}$

$\begin{array}{llll}0.071 & 0.276 & 0.234\end{array}$

$\begin{array}{llll}0.070 & 0.279 & 0.254\end{array}$

$\begin{array}{llll}0.061 & 0.222 & 0.156\end{array}$

$\begin{array}{llll}0.082 & 0.192 & 0.169\end{array}$

$\begin{array}{llll}0.074 & 0.219 & 0.170\end{array}$

$\begin{array}{llll}0.068 & 0.151 & 0.114\end{array}$

$\begin{array}{llll}0.063 & 0.161 & 0.118\end{array}$

$\begin{array}{llll}0.070 & 0.103 & 0.102\end{array}$

$\begin{array}{llll}0.048 & 0.111 & 0.096\end{array}$

$\begin{array}{lll}0.043 & 0.118 & 0.097\end{array}$

$\begin{array}{llll}0.056 & 0.173 & 0.117\end{array}$

$\begin{array}{lll}0.056 & 0.135 & 0.105\end{array}$

$\begin{array}{llll}0.049 & 0.111 & 0.085\end{array}$

$\begin{array}{llll}0.056 & 0.098 & 0.094\end{array}$

$\begin{array}{llll}0.046 & 0.134 & 0.094\end{array}$

$\begin{array}{lll}0.060 & 0.282 & 0.208\end{array}$

$\begin{array}{llll}0.055 & 0.275 & 0.196\end{array}$

$\begin{array}{llll}0.054 & 0.249 & 0.173\end{array}$

$\begin{array}{llll}0.084 & 0.124 & 0.111\end{array}$

$\begin{array}{llll}0.089 & 0.124 & 0.111\end{array}$

$\begin{array}{lll}0.085 & 0.132 & 0.120\end{array}$

$\begin{array}{llll}0.070 & 0.185 & 0.125\end{array}$

$\begin{array}{llll}0.063 & 0.172 & 0.103\end{array}$

$\begin{array}{llll}0.067 & 0.169 & 0.113\end{array}$

$\begin{array}{lll}0.106 & 0.125 & 0.159 \\ 0.062 & 0.093 & 0.185\end{array}$

99.

99.2

101.1

100.5

98.8

99.5

99.5

100.5

99.7

97.4

90.2

06.

106.

00.3

100.0

99.3
99.8

98.5

100.8

100.7

100.2

99.5

100.2

99.6

99.

100.2 
45938.06 $460 \quad 38.22$ 46138.43 $\begin{array}{lll}462 & 38.34\end{array}$ $463 \quad 38.57$ $464 \quad 38.75$ 46438.75 46638.93 $\begin{array}{lll}67 & 38.73\end{array}$ $\begin{array}{ll}68 & 38.59\end{array}$ $469 \quad 38.90$ $470 \quad 38.72$ 47138.63 $472 \quad 38.45$ $473 \quad 38.54$ $474 \quad 38.71$ 47438.71 $475 \quad 38.56$ $476 \quad 38.17$ $477 \quad 38.72$ $478 \quad 38.09$ $479 \quad 38.49$ $480 \quad 38.43$ 48138.70 $\begin{array}{lll}482 & 37.17\end{array}$ $485 \quad 37.33$ $487 \quad 38.10$ $488 \quad 38.09$ $489 \quad 38.67$ $490 \quad 38.41$ $491 \quad 39.12$ $492 \quad 37.99$ $493 \quad 38.60$ $494 \quad 38.45$ $495 \quad 38.80$ $496 \quad 38.36$ 49738.16 $498 \quad 38.52$ $499 \quad 38.10$ $500 \quad 38.26$ 50038.26 Hagan, $\begin{array}{lll}503 & 38.13\end{array}$ $504 \quad 38.78$ $505 \quad 39.10$ $\begin{array}{lll}506 & 38.38\end{array}$ $\begin{array}{llllllll}3.57 & 18.04 & 0.009 & 0.209 & 0.051 & 0.076 & 0.130 & 0.385\end{array}$ $\begin{array}{llllllll}3.60 & 18.35 & 0.019 & 0.208 & 0.120 & 0.044 & 0.143 & 0.090\end{array}$ $\begin{array}{lllllllll}3.57 & 18.27 & 0.018 & 0.198 & 0.093 & 0.049 & 0.156 & 0.087\end{array}$ $\begin{array}{lllllllll}3.53 & 18.32 & 0.021 & 0.201 & 0.114 & 0.047 & 0.119 & 0.090\end{array}$ $\begin{array}{lllllllll}3.44 & 18.21 & 0.010 & 0.221 & 0.072 & 0.063 & 0.128 & 0.154\end{array}$ $\begin{array}{lllllllll}3.49 & 18.21 & 0.009 & 0.244 & 0.069 & 0.070 & 0.116 & 0.169\end{array}$ $\begin{array}{lllllllll}3.54 & 18.27 & 0.008 & 0.231 & 0.061 & 0.075 & 0.120 & 0.148\end{array}$ $\begin{array}{lllllllll}3.34 & 18.37 & 0.013 & 0.232 & 0.086 & 0.063 & 0.091 & 0.117\end{array}$ $\begin{array}{lllllllll}3.56 & 18.35 & 0.008 & 0.219 & 0.077 & 0.067 & 0.111 & 0.114\end{array}$ $\begin{array}{llllllll}3.54 & 18.27 & 0.011 & 0.234 & 0.090 & 0.078 & 0.092 & 0.120\end{array}$ $\begin{array}{llllllll}3.61 & 18.54 & 0.013 & 0.231 & 0.083 & 0.061 & 0.187 & 0.123\end{array}$ $\begin{array}{llllllll}3.57 & 18.10 & 0.013 & 0.245 & 0.092 & 0.061 & 0.192 & 0.140\end{array}$ $\begin{array}{lllllllll}3.30 & 18.20 & 0.014 & 0.232 & 0.103 & 0.058 & 0.238 & 0.166\end{array}$ $\begin{array}{llllllll}3.46 & 18.08 & 0.018 & 0.235 & 0.122 & 0.056 & 0.113 & 0.097\end{array}$ $\begin{array}{llllllll}3.46 & 18.15 & 0.019 & 0.222 & 0.101 & 0.050 & 0.143 & 0.090\end{array}$ $\begin{array}{lllllllll}3.62 & 18.44 & 0.019 & 0.241 & 0.116 & 0.053 & 0.133 & 0.107\end{array}$ $\begin{array}{llllllll}3.61 & 18.35 & 0.069 & 0.177 & 0.165 & 0.040 & 0.136 & 0.072\end{array}$ $\begin{array}{llllllll}3.53 & 18.11 & 0.073 & 0.178 & 0.159 & 0.037 & 0.089 & 0.075\end{array}$ $\begin{array}{llllllll}3.45 & 18.03 & 0.069 & 0.190 & 0.148 & 0.040 & 0.105 & 0.069\end{array}$ $\begin{array}{lllllllll}3.64 & 17.82 & 0.017 & 0.226 & 0.127 & 0.048 & 0.123 & 0.107\end{array}$ $\begin{array}{lllllllll}3.43 & 18.33 & 0.019 & 0.236 & 0.102 & 0.054 & 0.299 & 0.203\end{array}$ $\begin{array}{lllllllll}3.58 & 17.85 & 0.018 & 0.234 & 0.125 & 0.052 & 0.111 & 0.090\end{array}$ $\begin{array}{lllllllll}3.62 & 18.30 & 0.009 & 0.231 & 0.066 & 0.069 & 0.144 & 0.184\end{array}$ $\begin{array}{lllllllll}3.44 & 17.85 & 0.009 & 0.229 & 0.077 & 0.073 & 0.111 & 0.163\end{array}$ $\begin{array}{lllllllll}3.50 & 17.91 & 0.010 & 0.241 & 0.073 & 0.060 & 0.131 & 0.161\end{array}$ $\begin{array}{llllllll}3.62 & 18.22 & 0.076 & 0.187 & 0.195 & 0.044 & 0.114 & 0.098\end{array}$ $\begin{array}{llllllll}3.65 & 18.82 & 0.071 & 0.192 & 0.195 & 0.041 & 0.096 & 0.081\end{array}$ $\begin{array}{llllllll}3.42 & 17.91 & 0.075 & 0.183 & 0.195 & 0.039 & 0.149 & 0.108\end{array}$ $\begin{array}{lllllllll}3.57 & 18.04 & 0.008 & 0.221 & 0.055 & 0.086 & 0.126 & 0.162\end{array}$ $\begin{array}{lllllllll}3.49 & 18.45 & 0.009 & 0.217 & 0.069 & 0.092 & 0.105 & 0.158\end{array}$ $\begin{array}{lllllllll}3.54 & 18.20 & 0.008 & 0.230 & 0.059 & 0.085 & 0.121 & 0.164\end{array}$ $\begin{array}{lllllllll}3.59 & 17.98 & 0.080 & 0.173 & 0.209 & 0.055 & 0.142 & 0.108\end{array}$ $\begin{array}{lllllllll}3.69 & 18.35 & 0.077 & 0.195 & 0.217 & 0.048 & 0.117 & 0.095\end{array}$ $\begin{array}{llllllll}3.43 & 17.74 & 0.079 & 0.172 & 0.209 & 0.055 & 0.128 & 0.100\end{array}$ $\begin{array}{lllllllll}3.58 & 18.20 & 0.020 & 0.240 & 0.103 & 0.052 & 0.227 & 0.154\end{array}$ $\begin{array}{lllllllll}3.62 & 18.17 & 0.019 & 0.255 & 0.117 & 0.051 & 0.256 & 0.195\end{array}$ $\begin{array}{llllllll}3.43 & 18.33 & 0.018 & 0.251 & 0.113 & 0.049 & 0.248 & 0.144\end{array}$ $\begin{array}{lllllllll}3.55 & 18.44 & 0.018 & 0.242 & 0.121 & 0.055 & 0.289 & 0.237\end{array}$ $\begin{array}{lllllllll}3.51 & 18.50 & 0.020 & 0.253 & 0.151 & 0.045 & 0.270 & 0.206\end{array}$ $\begin{array}{lllllllll}3.48 & 18.14 & 0.018 & 0.238 & 0.118 & 0.058 & 0.267 & 0.206\end{array}$ $\begin{array}{lllllllll}3.40 & 18.33 & 0.008 & 0.240 & 0.062 & 0.068 & 0.181 & 0.225\end{array}$ $\begin{array}{lllllllll}3.34 & 18.34 & 0.009 & 0.252 & 0.068 & 0.075 & 0.182 & 0.241\end{array}$ $\begin{array}{llllllll}3.65 & 17.70 & 0.010 & 0.241 & 0.066 & 0.065 & 0.172 & 0.222\end{array}$ inia Millbrig \#6 - Bottom of bed

$\begin{array}{llllllll}3.48 & 18.37 & 0.009 & 0.226 & 0.067 & 0.082 & 0.105 & 0.155\end{array}$ $\begin{array}{lllllllll}3.49 & 18.26 & 0.009 & 0.243 & 0.048 & 0.086 & 0.094 & 0.137\end{array}$ $\begin{array}{llllllll}3.73 & 18.39 & 0.009 & 0.227 & 0.072 & 0.084 & 0.097 & 0.147\end{array}$ $\begin{array}{lllllllll}3.57 & 18.17 & 0.008 & 0.224 & 0.055 & 0.087 & 0.136 & 0.155\end{array}$ $\begin{array}{lllllllll}3.50 & 18.38 & 0.010 & 0.220 & 0.076 & 0.089 & 0.115 & 0.160\end{array}$
$3.51 \quad 3.60$

3.57

$53.68 \quad 3.53$

$54.00 \quad 3.44$

$54.25 \quad 3.49$

54.42

54.50

$54.22 \quad 3.56$

$54.03 \quad 3.54$

$54.46 \quad 3.61$

5.21

$\begin{array}{ll}54.08 & 3.30\end{array}$

$53.83 \quad 3.46$

$53.96 \quad 3.46$

$54.19 \quad 3.62$

$53.98 \quad 3.61$

$53.44 \quad 3.53$

$\begin{array}{ll}54.21 & 3.45 \\ 53.33 & 3.64\end{array}$

$53.33 \quad 3.64$

$53.89 \quad 3.43$

$53.80 \quad 3.58$

$54.18 \quad 3.62$

$52.04 \quad 3.44$

$53.21 \quad 3.50$

$54.35 \quad 3.62$

$56.46 \quad 3.65$

$53.03 \quad 3.42$

$53.34 \quad 3.57$

$53.33 \quad 3.49$

$\begin{array}{ll}54.14 & 3.54 \\ 53.77 & 3.59\end{array}$

$54.77 \quad 3.69$

$53.19 \quad 3.43$

$54.04 \quad 3.58$

$53.83 \quad 3.62$

$54.32 \quad 3.43$

$53.70 \quad 3.55$

$53.42 \quad 3.51$

$\begin{array}{ll}53.93 & 3.48\end{array}$

$\begin{array}{ll}53.34 & 3.40 \\ 53.56 & 3.34\end{array}$

$53.58 \quad 3.34$

$\begin{array}{ll}52.93 & 3.48\end{array}$

$53.38 \quad 3.49$

$54.29 \quad 3.73$

$\begin{array}{ll}54.74 & 3.57\end{array}$

$53.73 \quad 3.50$ $\begin{array}{llllllll}1.34 & 0.014 & 0.209 & 0.065 & 0.098 & 0.152 & 0.489\end{array}$ $\begin{array}{llllllll}4.05 & 0.031 & 0.208 & 0.154 & 0.056 & 0.167 & 0.115\end{array}$ $\begin{array}{llllllll}41.87 & 0.031 & 0.208 & 0.154 & 0.056 & 0.167 & 0.115 \\ 4.198 & 0.198 & 0.120 & 0.063 & 0.183 & 0.110\end{array}$ $\begin{array}{llllllll}41.98 & 0.034 & 0.201 & 0.147 & 0.061 & 0.139 & 0.115\end{array}$ $\begin{array}{llllllll}41.73 & 0.016 & 0.221 & 0.092 & 0.082 & 0.150 & 0.196\end{array}$ $\begin{array}{llllllll}41.73 & 0.014 & 0.244 & 0.089 & 0.091 & 0.136 & 0.215\end{array}$ $\begin{array}{llllllll}41.87 & 0.013 & 0.231 & 0.078 & 0.097 & 0.141 & 0.188\end{array}$ $\begin{array}{lllllllll}41.73 & 0.014 & 0.244 & 0.089 & 0.091 & 0.136 & 0.215 \\ 42.10 & 0.0131 & 0.231 & 0.078 & 0.097 & 0.141 & 0.188\end{array}$ $\begin{array}{llllllll}41.87 & 0.013 & 0.231 & 0.078 & 0.097 & 0.141 & 0.188 \\ 2.10 & 0.021 & 0.232 & 0.111 & 0.081 & 0.106 & 0.148\end{array}$ $\begin{array}{lllllllll}4.05 & 0.013 & 0.219 & 0.099 & 0.087 & 0.130 & 0.144\end{array}$ $\begin{array}{llllllll}41.87 & 0.017 & 0.234 & 0.116 & 0.101 & 0.108 & 0.153\end{array}$ $\begin{array}{lllllllll}42.49 & 0.021 & 0.231 & 0.107 & 0.079 & 0.219 & 0.156\end{array}$ $\begin{array}{llllllll}41.48 & 0.021 & 0.245 & 0.118 & 0.079 & 0.225 & 0.178\end{array}$ $\begin{array}{llllllll}41.71 & 0.022 & 0.232 & 0.132 & 0.074 & 0.279 & 0.211\end{array}$ $\begin{array}{llllllll}41.43 & 0.030 & 0.235 & 0.157 & 0.072 & 0.133 & 0.123\end{array}$ $\begin{array}{llllllll}41.59 & 0.031 & 0.222 & 0.130 & 0.065 & 0.167 & 0.114\end{array}$ $\begin{array}{lllllllll}4.26 & 0.031 & 0.222 & 0.130 & 0.065 & 0.167 & 0.114\end{array}$ $\begin{array}{llllllll}42.05 & 0.114 & 0.177 & 0.212 & 0.052 & 0.160 & 0.091\end{array}$ $\begin{array}{lllllll}41.50 & 0.120 & 0.178 & 0.204 & 0.048 & 0.104 & 0.095\end{array}$ $\begin{array}{llllllll}1.32 & 0.114 & 0.190 & 0.191 & 0.052 & 0.123 & 0.087\end{array}$ $\begin{array}{llllllll}0.84 & 0.028 & 0.226 & 0.163 & 0.061 & 0.144 & 0.136\end{array}$ $\begin{array}{llllllll}42.00 & 0.032 & 0.236 & 0.131 & 0.070 & 0.350 & 0.258\end{array}$ $\begin{array}{llllllll}40.90 & 0.030 & 0.234 & 0.160 & 0.067 & 0.130 & 0.114\end{array}$ $\begin{array}{llllllll}41.94 & 0.015 & 0.231 & 0.085 & 0.089 & 0.168 & 0.233\end{array}$ $\begin{array}{llllllll}40.90 & 0.014 & 0.229 & 0.099 & 0.094 & 0.130 & 0.207\end{array}$ $\begin{array}{llll}0.078 & 0.153 & 0.205\end{array}$ $\begin{array}{lllll}1.04 & 0.016 & 0.241 & 0.094\end{array}$ $\begin{array}{llllllllll}43.13 & 0.117 & 0.187 & 0.250 & 0.056 & 0.134 & 0.124\end{array}$ $\begin{array}{lllllll}41.04 & 0.124 & 0.183 & 0.251 & 0.051 & 0.175 & 0.138\end{array}$ $\begin{array}{llllllll}41.34 & 0.014 & 0.221 & 0.071 & 0.111 & 0.148 & 0.205\end{array}$ $\begin{array}{llllllll}42.28 & 0.014 & 0.217 & 0.088 & 0.118 & 0.123 & 0.201\end{array}$ $\begin{array}{llllllll}41.71 & 0.013 & 0.230 & 0.076 & 0.110 & 0.142 & 0.208\end{array}$ $\begin{array}{llllllll}41.20 & 0.133 & 0.173 & 0.269 & 0.070 & 0.166 & 0.138\end{array}$ $\begin{array}{llllllll}41.20 & 0.133 & 0.173 & 0.269 & 0.070 & 0.166 & 0.138\end{array}$ $\begin{array}{llllllll}40.65 & 0.131 & 0.172 & 0.269 & 0.070 & 0.149 & 0.127\end{array}$ $\begin{array}{llllllll}41.71 & 0.033 & 0.240 & 0.132 & 0.067 & 0.266 & 0.195\end{array}$ $\begin{array}{llllllll}41.64 & 0.031 & 0.255 & 0.151 & 0.065 & 0.299 & 0.247\end{array}$ $\begin{array}{llllllll}4.00 & 0.030 & 0.251 & 0.146 & 0.064 & 0.290 & 0.183\end{array}$ $\begin{array}{llllllll}42.26 & 0.031 & 0.242 & 0.155 & 0.070 & 0.338 & 0.301\end{array}$ $\begin{array}{llllllll}42.39 & 0.032 & 0.253 & 0.194 & 0.058 & 0.316 & 0.261\end{array}$ $\begin{array}{llllllll}41.57 & 0.030 & 0.238 & 0.152 & 0.075 & 0.312 & 0.261\end{array}$ $\begin{array}{llllllll}42.00 & 0.014 & 0.240 & 0.080 & 0.088 & 0.212 & 0.286\end{array}$ $\begin{array}{llllllll}42.03 & 0.015 & 0.252 & 0.087 & 0.097 & 0.213 & 0.306\end{array}$ $\begin{array}{lllllll}40.56 & 0.017 & 0.241 & 0.085 & 0.084 & 0.202 & 0.282\end{array}$

$\begin{array}{lllllll}42.10 & 0.015 & 0.226 & 0.086 & 0.106 & 0.123 & 0.197\end{array}$ $\begin{array}{llllllll}41.84 & 0.015 & 0.243 & 0.061 & 0.111 & 0.110 & 0.174\end{array}$ $\begin{array}{llllllll}42.14 & 0.016 & 0.227 & 0.092 & 0.108 & 0.114 & 0.187\end{array}$ $\begin{array}{lllllll}41.64 & 0.013 & 0.224 & 0.070 & 0.113 & 0.159 & 0.197\end{array}$ $\begin{array}{llllllll}42.12 & 0.016 & 0.220 & 0.098 & 0.115 & 0.134 & 0.203\end{array}$
99.2

99.9

99.9

100.3

100.3

00.

100.6

100.5

101.4

100.1

100.0

99.5

100.9

99.2

99.7

98.6

100.4

99.0

100.6

97.2

100.6

104.1

98.4
99.0

99.9

100.2

99.5

101.4

98.2

00.3

100.1

100.7

100.6

100.4

100.0

98.7

99.3

99.4

100.9

100.7

100.1 
$507 \quad 36.18$ $508 \quad 0.00$
509 50937.99 $\begin{array}{lll}510 & 38.21\end{array}$ $\begin{array}{lll}511 & 37.61\end{array}$ $512 \quad 38.29$ $512 \quad 38.29$ $\begin{array}{lll}513 & 38.28\end{array}$ $\begin{array}{lll}514 & 38.35\end{array}$ $\begin{array}{lll}15 & 38.32\end{array}$ $\begin{array}{lll}516 & 38.34\end{array}$ $517 \quad 38.14$ $518 \quad 38.26$ $519 \quad 38.26$ $520 \quad 38.85$ 52138.03 $\begin{array}{lll}522 & 38.34\end{array}$ $\begin{array}{lll}523 & 38.37\end{array}$ 52438.51 $525 \quad 38.49$ $\begin{array}{lll}526 & 37.79\end{array}$ $527 \quad 38.04$ $\begin{array}{lll}528 & 37.91\end{array}$ $\begin{array}{lll}529 & 38.17\end{array}$ $\begin{array}{lll}530 & 38.07\end{array}$ 53138.02 $\begin{array}{lll}532 & 38.04\end{array}$ $\begin{array}{lll}533 & 37.87\end{array}$ $\begin{array}{lll}534 & 38.28\end{array}$ 53537.96 $\begin{array}{lll}536 & 38.25\end{array}$ $\begin{array}{lll}537 & 37.83\end{array}$ $\begin{array}{lll}538 & 38.38\end{array}$ $539 \quad 38.16$ 53938.16 $\begin{array}{lll}540 & 38.15\end{array}$ $\begin{array}{lll}541 & 37.95\end{array}$ $\begin{array}{lll}542 & 37.94\end{array}$ $543 \quad 37.81$ $544 \quad 38.32$ $\begin{array}{lll}545 & 38.29\end{array}$ 54638.06 $547 \quad 38.29$ $548 \quad 38.29$
58.56 54938.47 $\begin{array}{lll}550 & 38.24\end{array}$ $\begin{array}{lll}551 & 38.28\end{array}$ $552 \quad 38.40$ $\begin{array}{lll}553 & 38.28\end{array}$ $\begin{array}{llllllll}3.37 & 16.87 & 0.009 & 0.239 & 0.066 & 0.078 & 0.143 & 0.152\end{array}$ $\begin{array}{lllllllll}0.00 & 0.00 & 0.000 & 0.000 & 0.000 & 0.000 & 0.000 & 0.000\end{array}$ $\begin{array}{llllllll}3.55 & 18.18 & 0.011 & 0.233 & 0.080 & 0.068 & 0.096 & 0.119\end{array}$ $\begin{array}{lllllllll}3.52 & 18.15 & 0.009 & 0.231 & 0.075 & 0.068 & 0.118 & 0.122\end{array}$ $\begin{array}{lllllllll}3.49 & 17.67 & 0.019 & 0.277 & 0.104 & 0.076 & 0.097 & 0.122\end{array}$ $\begin{array}{lllllllll}3.53 & 18.45 & 0.009 & 0.226 & 0.066 & 0.089 & 0.171 & 0.244\end{array}$ $\begin{array}{lllllllll}3.48 & 18.28 & 0.007 & 0.248 & 0.059 & 0.084 & 0.146 & 0.247\end{array}$ $\begin{array}{lllllllll}3.68 & 18.51 & 0.008 & 0.230 & 0.061 & 0.078 & 0.170 & 0.228\end{array}$ $\begin{array}{llllllll}3.57 & 18.32 & 0.010 & 0.255 & 0.084 & 0.069 & 0.159 & 0.207\end{array}$ $\begin{array}{lllllllll}3.59 & 18.15 & 0.010 & 0.239 & 0.067 & 0.071 & 0.190 & 0.207\end{array}$ $\begin{array}{lllllllll}3.51 & 18.38 & 0.011 & 0.240 & 0.074 & 0.066 & 0.149 & 0.194\end{array}$ $\begin{array}{lllllllll}3.48 & 18.29 & 0.017 & 0.272 & 0.096 & 0.072 & 0.124 & 0.085\end{array}$ $\begin{array}{lllllllll}3.55 & 18.41 & 0.017 & 0.271 & 0.115 & 0.077 & 0.140 & 0.082\end{array}$ $\begin{array}{lllllllll}3.58 & 18.01 & 0.017 & 0.263 & 0.092 & 0.068 & 0.154 & 0.084\end{array}$ $\begin{array}{lllllllll}3.54 & 18.26 & 0.014 & 0.278 & 0.087 & 0.072 & 0.204 & 0.120\end{array}$ $\begin{array}{lllllllll}3.36 & 18.28 & 0.015 & 0.273 & 0.074 & 0.064 & 0.222 & 0.111\end{array}$ $\begin{array}{lllllllll}3.42 & 18.29 & 0.016 & 0.266 & 0.076 & 0.072 & 0.231 & 0.139\end{array}$ $\begin{array}{lllllllll}3.53 & 18.52 & 0.009 & 0.248 & 0.064 & 0.068 & 0.118 & 0.152\end{array}$ $\begin{array}{lllllllll}3.46 & 17.95 & 0.010 & 0.254 & 0.079 & 0.073 & 0.114 & 0.171\end{array}$ $\begin{array}{llllllll}3.65 & 18.03 & 0.009 & 0.237 & 0.048 & 0.071 & 0.127 & 0.178\end{array}$ $\begin{array}{lllllllll}3.47 & 18.21 & 0.011 & 0.223 & 0.080 & 0.070 & 0.138 & 0.188\end{array}$ $\begin{array}{lllllllll}3.55 & 18.42 & 0.008 & 0.245 & 0.069 & 0.074 & 0.130 & 0.148\end{array}$ $\begin{array}{lllllllll}3.48 & 18.43 & 0.011 & 0.226 & 0.077 & 0.077 & 0.083 & 0.100\end{array}$ $\begin{array}{lllllllll}3.47 & 18.37 & 0.008 & 0.238 & 0.064 & 0.078 & 0.144 & 0.212\end{array}$ $\begin{array}{lllllllll}3.43 & 18.35 & 0.008 & 0.240 & 0.057 & 0.091 & 0.070 & 0.144\end{array}$ $\begin{array}{lllllllll}3.66 & 18.31 & 0.009 & 0.225 & 0.065 & 0.082 & 0.093 & 0.125\end{array}$ $\begin{array}{lllllllll}3.53 & 18.36 & 0.010 & 0.234 & 0.077 & 0.070 & 0.114 & 0.188\end{array}$ $\begin{array}{llllllll}3.56 & 18.40 & 0.009 & 0.234 & 0.060 & 0.066 & 0.149 & 0.189\end{array}$ $\begin{array}{lllllllll}3.60 & 18.45 & 0.010 & 0.245 & 0.075 & 0.074 & 0.120 & 0.182\end{array}$ $\begin{array}{lllllllll}3.55 & 18.53 & 0.011 & 0.229 & 0.065 & 0.076 & 0.163 & 0.195\end{array}$ $\begin{array}{lllllllll}3.72 & 18.50 & 0.011 & 0.233 & 0.085 & 0.074 & 0.148 & 0.215\end{array}$ $\begin{array}{lllllllll}3.60 & 18.45 & 0.011 & 0.236 & 0.061 & 0.068 & 0.178 & 0.211\end{array}$ $\begin{array}{lllllllll}3.46 & 18.13 & 0.011 & 0.255 & 0.082 & 0.073 & 0.145 & 0.182\end{array}$ $\begin{array}{lllllllll}3.60 & 18.49 & 0.010 & 0.240 & 0.065 & 0.064 & 0.148 & 0.137\end{array}$ $\begin{array}{lllllllll}3.42 & 18.35 & 0.009 & 0.242 & 0.079 & 0.070 & 0.102 & 0.133\end{array}$ $\begin{array}{lllllllll}3.46 & 18.18 & 0.008 & 0.243 & 0.065 & 0.071 & 0.099 & 0.110\end{array}$ $\begin{array}{lllllllll}3.41 & 18.30 & 0.009 & 0.235 & 0.079 & 0.069 & 0.093 & 0.115\end{array}$ $\begin{array}{llllllll}3.64 & 18.36 & 0.009 & 0.230 & 0.065 & 0.070 & 0.132 & 0.121\end{array}$ $\begin{array}{llllllll}3.69 & 18.34 & 0.022 & 0.228 & 0.115 & 0.054 & 0.133 & 0.087\end{array}$ $\begin{array}{lllllllll}3.51 & 18.14 & 0.016 & 0.223 & 0.105 & 0.043 & 0.149 & 0.081\end{array}$ $\begin{array}{llllllll}3.48 & 18.33 & 0.018 & 0.254 & 0.124 & 0.048 & 0.115 & 0.094\end{array}$ $\begin{array}{lllllllll}3.48 & 18.26 & 0.009 & 0.235 & 0.066 & 0.080 & 0.113 & 0.144\end{array}$ $\begin{array}{lllllllll}3.68 & 18.43 & 0.010 & 0.232 & 0.069 & 0.071 & 0.113 & 0.150\end{array}$ $\begin{array}{lllllllll}3.68 & 18.45 & 0.009 & 0.235 & 0.063 & 0.068 & 0.138 & 0.144\end{array}$ $\begin{array}{lllllllll}3.48 & 18.17 & 0.018 & 0.243 & 0.118 & 0.060 & 0.154 & 0.111\end{array}$ $\begin{array}{lllllllll}3.63 & 18.60 & 0.015 & 0.223 & 0.096 & 0.051 & 0.164 & 0.108\end{array}$ $\begin{array}{llllllll}3.48 & 18.13 & 0.019 & 0.240 & 0.116 & 0.059 & 0.160 & 0.119\end{array}$ $\begin{array}{lllllllll}3.56 & 18.34 & 0.018 & 0.229 & 0.093 & 0.051 & 0.149 & 0.089\end{array}$ $\begin{array}{llllllll}3.51 & 18.35 & 0.019 & 0.239 & 0.122 & 0.057 & 0.206 & 0.143\end{array}$
$50.65 \quad 3.37$ $\begin{array}{lllll}38.66 & 0.016 & 0.239 & 0.085\end{array}$ $\begin{array}{llll}0.00 & 0.000 & 0.000 & 0.000\end{array}$ $\begin{array}{llll}41.66 & 0.019 & 0.233 & 0.102\end{array}$ $\begin{array}{lllll}41.59 & 0.015 & 0.231 & 0.096\end{array}$ $\begin{array}{lllll}40.49 & 0.032 & 0.277 & 0.134\end{array}$ $\begin{array}{lllll}42.28 & 0.014 & 0.226 & 0.085\end{array}$ $\begin{array}{lllll}41.89 & 0.011 & 0.248 & 0.076\end{array}$ $\begin{array}{lllll}4.89 & 0.011 & 0.248 & 0.076\end{array}$ $\begin{array}{llll}42.42 & 0.014 & 0.230 & 0.078\end{array}$ $\begin{array}{llll}41.98 & 0.017 & 0.255 & 0.108 \\ 41.59 & 0.016 & 0.239 & 0.086\end{array}$ $\begin{array}{llll}41.59 & 0.016 & 0.239 & 0.08\end{array}$ $\begin{array}{lllll}42.12 & 0.018 & 0.240 & 0.095\end{array}$ $\begin{array}{lllll}41.91 & 0.029 & 0.272 & 0.124\end{array}$ $\begin{array}{lllll}42.19 & 0.028 & 0.271 & 0.148\end{array}$ $\begin{array}{lllll}41.27 & 0.029 & 0.263 & 0.118\end{array}$ $\begin{array}{lllll}41.84 & 0.024 & 0.278 & 0.112\end{array}$ $\begin{array}{lllll}41.89 & 0.026 & 0.273 & 0.095\end{array}$ $\begin{array}{llll}41.89 & 0.026 & 0.273 & 0.095 \\ 41.91 & 0.027 & 0.266 & 0.098\end{array}$ $\begin{array}{lllll}41.91 & 0.027 & 0.266 & 0.098\end{array}$ $\begin{array}{lllll}41.13 & 0.017 & 0.254 & 0.102\end{array}$ $\begin{array}{lllll}41.32 & 0.016 & 0.237 & 0.062\end{array}$ $\begin{array}{lllll}41.73 & 0.019 & 0.223 & 0.103\end{array}$ $\begin{array}{lllll}42.21 & 0.014 & 0.245 & 0.088\end{array}$ $\begin{array}{lllll}42.23 & 0.018 & 0.226 & 0.099\end{array}$ $\begin{array}{lllll}42.10 & 0.014 & 0.238 & 0.082\end{array}$ $\begin{array}{lllll}42.05 & 0.013 & 0.240 & 0.073\end{array}$ $\begin{array}{llll}42.05 & 0.013 & 0.240 & 0.073\end{array}$ $\begin{array}{llll}41.96 & 0.015 & 0.225 & 0.08\end{array}$ $\begin{array}{lllll}42.07 & 0.016 & 0.234 & 0.099\end{array}$ $\begin{array}{lllll}42.16 & 0.015 & 0.234 & 0.077\end{array}$ $\begin{array}{lllll}42.28 & 0.017 & 0.245 & 0.097\end{array}$ $\begin{array}{lllll}42.46 & 0.018 & 0.229 & 0.084\end{array}$ $\begin{array}{lllll}42.39 & 0.018 & 0.233 & 0.109\end{array}$ $\begin{array}{lllll}42.28 & 0.017 & 0.236 & 0.079\end{array}$

41.55 $\begin{array}{lllll}42.05 & 0.016 & 0.242 & 0.101\end{array}$ $\begin{array}{llll}41.66 & 0.013 & 0.242 & 0.101\end{array}$ 41.94 $\begin{array}{lll}0.101 & 0.167 & 0.193\end{array}$ $\begin{array}{lll}0.000 & 0.000 & 0.000\end{array}$ $\begin{array}{lll}0.088 & 0.112 & 0.151\end{array}$ $\begin{array}{lll}0.088 & 0.138 & 0.155 \\ 0.099 & 0.113 & 0.155\end{array}$ $\begin{array}{lll}0.099 & 0.113 & 0.155\end{array}$ $\begin{array}{llll}0.115 & 0.201 & 0.309\end{array}$ $\begin{array}{llll}0.109 & 0.171 & 0.314\end{array}$ $\begin{array}{lll}0.100 & 0.199 & 0.290\end{array}$ $\begin{array}{llll}0.089 & 0.186 & 0.262\end{array}$ $\begin{array}{llll}0.091 & 0.222 & 0.263\end{array}$ $\begin{array}{lll}0.085 & 0.175 & 0.247\end{array}$ $\begin{array}{llll}0.093 & 0.145 & 0.108\end{array}$ $\begin{array}{llll}0.099 & 0.164 & 0.104\end{array}$ $\begin{array}{llll}0.088 & 0.180 & 0.107\end{array}$ $\begin{array}{llll}0.083 & 0.259 & 0.141\end{array}$ $\begin{array}{llll}0.083 & 0.259 & 0.141\end{array}$ $\begin{array}{llll}0.088 & 0.138 & 0.193\end{array}$ $\begin{array}{llll}0.094 & 0.133 & 0.218\end{array}$ $\begin{array}{llll}0.092 & 0.148 & 0.226\end{array}$ $\begin{array}{llll}0.090 & 0.162 & 0.239\end{array}$ $\begin{array}{llll}0.095 & 0.152 & 0.188\end{array}$ $\begin{array}{llll}0.099 & 0.097 & 0.127\end{array}$ $\begin{array}{llll}0.101 & 0.169 & 0.269\end{array}$ $\begin{array}{lll}0.118 & 0.082 & 0.182\end{array}$ $\begin{array}{llll}0.106 & 0.109 & 0.159\end{array}$ $\begin{array}{llll}0.091 & 0.133 & 0.239\end{array}$ $\begin{array}{llll}0.085 & 0.174 & 0.240\end{array}$ $\begin{array}{lll}0.096 & 0.140 & 0.231\end{array}$ $\begin{array}{llll}0.097 & 0.191 & 0.247\end{array}$ $\begin{array}{llll}0.095 & 0.173 & 0.273\end{array}$ $\begin{array}{llll}0.087 & 0.208 & 0.267\end{array}$ $\begin{array}{llll}0.094 & 0.170 & 0.231\end{array}$ $\begin{array}{llll}0.083 & 0.173 & 0.174\end{array}$ $\begin{array}{llll}0.090 & 0.120 & 0.169\end{array}$ $\begin{array}{llll}0.092 & 0.116 & 0.139\end{array}$ $\begin{array}{llll}0.089 & 0.109 & 0.146\end{array}$ $\begin{array}{lll}0.090 & 0.155 & 0.153\end{array}$ $\begin{array}{llll}0.070 & 0.156 & 0.111\end{array}$ $\begin{array}{llll}0.055 & 0.175 & 0.103\end{array}$ $\begin{array}{llll}0.062 & 0.134 & 0.119\end{array}$ $\begin{array}{lll}0.104 & 0.132 & 0.182\end{array}$ $\begin{array}{llll}0.092 & 0.133 & 0.191\end{array}$ $\begin{array}{llll}0.088 & 0.161 & 0.183\end{array}$ $\begin{array}{lll}0.078 & 0.180 & 0.141\end{array}$ $\begin{array}{llll}0.066 & 0.192 & 0.137\end{array}$ $\begin{array}{llll}0.076 & 0.188 & 0.151\end{array}$ $\begin{array}{lll}0.066 & 0.174 & 0.113 \\ 0.074 & 0.241 & 0.182\end{array}$ 
55638.90 $557 \quad 38.55$ $\begin{array}{lll}558 & 38.47\end{array}$ $\begin{array}{lll}559 & 38.27\end{array}$ $\begin{array}{lll}560 & 38.42\end{array}$ $561 \quad 38.34$ $562 \quad 38.12$ $\begin{array}{lll}564 & 38.17\end{array}$ $\begin{array}{lll}565 & 38.21\end{array}$ $\begin{array}{lll}566 & 38.21\end{array}$ $567 \quad 38.16$ $\begin{array}{lll}568 & 37.96\end{array}$ $\begin{array}{lll}569 & 38.41\end{array}$ $570 \quad 38.32$ $571 \quad 38.05$ $573 \quad 38.95$ $575 \quad 38.16$ $\begin{array}{lll}576 & 38.03\end{array}$ $\begin{array}{lll}577 & 38.29\end{array}$ $\begin{array}{lll}578 & 37.91\end{array}$ $\begin{array}{lll}579 & 38.14\end{array}$ $580 \quad 38.42$ $\begin{array}{lll}581 & 38.10\end{array}$ $\begin{array}{lll}582 & 38.38\end{array}$ $\begin{array}{lll}583 & 38.27\end{array}$ $\begin{array}{lll}584 & 38.13\end{array}$ $\begin{array}{lll}585 & 38.17\end{array}$ Vasagå

$\begin{array}{ll}586 & 37.15 \\ 587 & 37.07\end{array}$ $\begin{array}{lll}587 & 37.07\end{array}$ $\begin{array}{lll}588 & 37.68\end{array}$ $\begin{array}{lll}589 & 37.48\end{array}$ $\begin{array}{lll}590 & 37.21\end{array}$ $\begin{array}{lll}591 & 37.84\end{array}$ $\begin{array}{lll}592 & 37.44\end{array}$ $593 \quad 37.82$ $\begin{array}{lll}594 & 37.27\end{array}$ $\begin{array}{lll}595 & 37.55\end{array}$ $596 \quad 37.35$ 59637.35 59737.36 $\begin{array}{lll}598 & 37.15\end{array}$ $599 \quad 37.48$ $600 \quad 38.24$ $601 \quad 38.37$ $602 \quad 38.57$ $603 \quad 37.91$ $\begin{array}{llllllll}3.61 & 18.22 & 0.018 & 0.233 & 0.120 & 0.056 & 0.128 & 0.094\end{array}$ $\begin{array}{llllllll}3.58 & 18.16 & 0.018 & 0.227 & 0.110 & 0.047 & 0.162 & 0.114\end{array}$ $\begin{array}{lllllllll}3.53 & 18.11 & 0.017 & 0.238 & 0.120 & 0.053 & 0.156 & 0.112\end{array}$ $\begin{array}{lllllllll}3.54 & 18.27 & 0.018 & 0.232 & 0.113 & 0.055 & 0.167 & 0.119\end{array}$ $\begin{array}{lllllllll}3.59 & 18.40 & 0.010 & 0.236 & 0.061 & 0.071 & 0.093 & 0.088\end{array}$ $\begin{array}{lllllllll}3.61 & 18.31 & 0.011 & 0.244 & 0.072 & 0.071 & 0.100 & 0.136\end{array}$ $\begin{array}{lllllllll}3.55 & 18.22 & 0.009 & 0.238 & 0.074 & 0.068 & 0.117 & 0.127\end{array}$ $\begin{array}{lllllllll}3.50 & 18.08 & 0.009 & 0.222 & 0.080 & 0.078 & 0.100 & 0.138 \\ 3.33 & 18.19 & 0.011 & 0.226 & 0.064 & 0.070 & 0.114 & 0.125 \\ 3.70 & 18.12 & 0.009 & 0.236 & 0.081 & 0.076 & 0.084 & 0.121\end{array}$ $\begin{array}{lllllllll}3.33 & 18.19 & 0.011 & 0.226 & 0.064 & 0.070 & 0.114 & 0.125 \\ 3.70 & 18.12 & 0.009 & 0.236 & 0.081 & 0.076 & 0.084 & 0.121\end{array}$ $\begin{array}{llllllll}3.37 & 17.92 & 0.018 & 0.232 & 0.113 & 0.054 & 0.138 & 0.076\end{array}$ $\begin{array}{lllllllll}3.58 & 18.15 & 0.020 & 0.220 & 0.120 & 0.051 & 0.117 & 0.094\end{array}$ $\begin{array}{lllllllll}3.59 & 17.62 & 0.021 & 0.238 & 0.103 & 0.050 & 0.138 & 0.092\end{array}$ $\begin{array}{lllllllll}3.58 & 18.03 & 0.010 & 0.238 & 0.092 & 0.074 & 0.097 & 0.132\end{array}$ $\begin{array}{lllllllll}3.42 & 18.09 & 0.010 & 0.227 & 0.065 & 0.072 & 0.132 & 0.134\end{array}$ $\begin{array}{lllllllll}3.60 & 18.42 & 0.014 & 0.226 & 0.082 & 0.068 & 0.086 & 0.122\end{array}$ $\begin{array}{lllllllll}3.44 & 17.95 & 0.012 & 0.253 & 0.073 & 0.066 & 0.117 & 0.141\end{array}$ $\begin{array}{llllllll}3.54 & 18.22 & 0.009 & 0.230 & 0.074 & 0.067 & 0.124 & 0.136\end{array}$ $\begin{array}{lllllllll}3.57 & 18.44 & 0.012 & 0.251 & 0.067 & 0.065 & 0.155 & 0.146\end{array}$ $\begin{array}{lllllllll}3.39 & 18.35 & 0.013 & 0.219 & 0.098 & 0.073 & 0.093 & 0.108\end{array}$ $\begin{array}{lllllllll}3.50 & 18.34 & 0.011 & 0.223 & 0.063 & 0.077 & 0.109 & 0.101\end{array}$ $\begin{array}{lllllllll}3.57 & 18.13 & 0.011 & 0.227 & 0.070 & 0.076 & 0.103 & 0.109\end{array}$ $\begin{array}{lllllllll}3.56 & 18.30 & 0.011 & 0.220 & 0.068 & 0.069 & 0.108 & 0.130\end{array}$ $\begin{array}{lllllllll}3.47 & 18.37 & 0.012 & 0.213 & 0.079 & 0.073 & 0.156 & 0.172\end{array}$ $\begin{array}{lllllllll}3.52 & 18.25 & 0.010 & 0.216 & 0.071 & 0.075 & 0.128 & 0.119\end{array}$ $\begin{array}{lllllllll}3.56 & 18.38 & 0.011 & 0.217 & 0.075 & 0.086 & 0.109 & 0.154\end{array}$ $\begin{array}{llllllll}3.59 & 18.28 & 0.008 & 0.228 & 0.059 & 0.084 & 0.120 & 0.152\end{array}$ $\begin{array}{lllllllll}3.61 & 18.37 & 0.010 & 0.207 & 0.069 & 0.088 & 0.094 & 0.162\end{array}$ $\begin{array}{llllllll}3.58 & 18.05 & 0.008 & 0.226 & 0.064 & 0.070 & 0.115 & 0.107\end{array}$ $\begin{array}{llllllll}3.55 & 17.99 & 0.011 & 0.228 & 0.091 & 0.076 & 0.140 & 0.148\end{array}$ nekulle \#1 - Top of bed

$\begin{array}{lllllllll}3.56 & 18.41 & 0.092 & 0.619 & 0.694 & 0.518 & 0.097 & 0.347\end{array}$ $\begin{array}{lllllllll}3.66 & 18.54 & 0.092 & 0.619 & 0.694 & 0.518 & 0.097 & 0.347\end{array}$ $\begin{array}{lllllllll}3.48 & 18.54 & 0.092 & 0.619 & 0.694 & 0.518 & 0.097 & 0.347\end{array}$ $\begin{array}{lllllllll}3.48 & 18.46 & 0.092 & 0.619 & 0.694 & 0.518 & 0.097 & 0.347\end{array}$ $\begin{array}{llllllll}3.76 & 18.45 & 0.092 & 0.619 & 0.694 & 0.518 & 0.097 & 0.347\end{array}$ $\begin{array}{llllllll}3.58 & 18.54 & 0.119 & 0.534 & 0.555 & 0.236 & 0.118 & 0.294\end{array}$ $\begin{array}{lllllllll}3.47 & 18.75 & 0.119 & 0.515 & 0.570 & 0.233 & 0.110 & 0.289\end{array}$ $\begin{array}{lllllllll}3.56 & 18.67 & 0.119 & 0.515 & 0.557 & 0.233 & 0.140 & 0.287\end{array}$ $\begin{array}{llllllll}3.68 & 18.38 & 0.094 & 0.549 & 0.626 & 0.382 & 0.125 & 0.358\end{array}$ $\begin{array}{lllllllll}3.45 & 18.74 & 0.095 & 0.568 & 0.620 & 0.391 & 0.105 & 0.333\end{array}$ $\begin{array}{lllllllll}3.58 & 18.39 & 0.093 & 0.551 & 0.636 & 0.390 & 0.092 & 0.335\end{array}$ $\begin{array}{lllllllll}3.59 & 18.54 & 0.086 & 0.606 & 0.666 & 0.508 & 0.100 & 0.332\end{array}$ $\begin{array}{lllllllll}3.52 & 18.64 & 0.086 & 0.639 & 0.700 & 0.514 & 0.094 & 0.346\end{array}$ $\begin{array}{llllllll}3.48 & 18.42 & 0.089 & 0.627 & 0.670 & 0.512 & 0.114 & 0.342\end{array}$ $\begin{array}{llllllll}3.51 & 18.14 & 0.039 & 0.840 & 0.157 & 0.119 & 0.187 & 0.172\end{array}$ $\begin{array}{llllllll}3.52 & 18.36 & 0.041 & 0.858 & 0.141 & 0.118 & 0.228 & 0.181\end{array}$ $\begin{array}{lllllllll}3.52 & 18.40 & 0.044 & 0.866 & 0.163 & 0.120 & 0.208 & 0.168\end{array}$ $\begin{array}{lllllllll}3.68 & 18.51 & 0.058 & 0.767 & 0.372 & 0.320 & 0.166 & 0.207\end{array}$
$54.46 \quad 3.61$

$53.97 \quad 3.58$

$53.86 \quad 3.53$

$\begin{array}{ll}53.58 & 3.54 \\ 53.79 & 3.59 \\ 53.68 & 3.61\end{array}$

$53.68 \quad 3.61$

$53.37 \quad 3.55$

$53.16 \quad 3.50$

$53.44 \quad 3.33$

$53.49 \quad 3.70$

$53.49 \quad 3.37$

$53.42 \quad 3.58$

53.14

$53.77 \quad 3.58$

$53.65 \quad 3.42$

$53.27 \quad 3.60$

$53.13 \quad 3.44$

$53.69 \quad 3.54$

$53.68 \quad 3.57$

$53.42 \quad 3.39$

$\begin{array}{ll}53.24 & 3.50\end{array}$

$53.61 \quad 3.57$

$53.07 \quad 3.56$

$53.40 \quad 3.47$

$53.79 \quad 3.52$

$53.34 \quad 3.56$

$53.73 \quad 3.59$

$\begin{array}{ll}53.58 & 3.61\end{array}$

$53.38 \quad 3.58$

$53.44 \quad 3.55$

$52.01 \quad 3.56$

$51.90 \quad 3.66$

$52.75 \quad 3.48$

$52.47 \quad 3.48$

$52.09 \quad 3.76$

$52.98 \quad 3.58$

$52.42 \quad 3.47$

$\begin{array}{ll}52.95 & 3.56 \\ 52.18 & 3.68\end{array}$

$52.57 \quad 3.45$

$52.29 \quad 3.58$

$52.30 \quad 3.59$

$52.01 \quad 3.52$

$52.47 \quad 3.48$

$53.54 \quad 3.51$

$53.72 \quad 3.52$

$54.00 \quad 3.52$

$53.07 \quad 3.68$ $\begin{array}{lllllll}1.75 & 0.029 & 0.233 & 0.154 & 0.072 & 0.150 & 0.119\end{array}$ $\begin{array}{llllllll}41.61 & 0.029 & 0.227 & 0.141 & 0.061 & 0.190 & 0.145\end{array}$ $\begin{array}{lllllll}41.50 & 0.029 & 0.238 & 0.155 & 0.069 & 0.182 & 0.142\end{array}$ $\begin{array}{llllllll}41.87 & 0.031 & 0.232 & 0.145 & 0.071 & 0.195 & 0.151\end{array}$ $\begin{array}{llllllll}42.16 & 0.016 & 0.236 & 0.079 & 0.091 & 0.108 & 0.111\end{array}$ $\begin{array}{llllllll}41.96 & 0.018 & 0.244 & 0.092 & 0.092 & 0.117 & 0.172\end{array}$ $\begin{array}{llllllll}41.75 & 0.015 & 0.238 & 0.095 & 0.088 & 0.137 & 0.162\end{array}$ $\begin{array}{llllllll}41.75 & 0.015 & 0.238 & 0.095 & 0.088 & 0.137 & 0.162\end{array}$ $\begin{array}{lllllll}41.43 & 0.015 & 0.222 & 0.103 & 0.101 & 0.118 & 0.175 \\ 41.68 & 0.017 & 0.226 & 0.083 & 0.091 & 0.133 & 0.159\end{array}$ $\begin{array}{llllllll}41.43 & 0.015 & 0.222 & 0.103 & 0.101 & 0.118 & 0.175 \\ 1.68 & 0.017 & 0.226 & 0.083 & 0.091 & 0.133 & 0.159\end{array}$ $\begin{array}{llllllll}41.52 & 0.015 & 0.236 & 0.104 & 0.098 & 0.098 & 0.153\end{array}$ $\begin{array}{llllllll}41.06 & 0.029 & 0.232 & 0.145 & 0.069 & 0.162 & 0.097\end{array}$ $\begin{array}{lllllll}41.59 & 0.033 & 0.220 & 0.154 & 0.066 & 0.137 & 0.119\end{array}$ $\begin{array}{llllllll}40.38 & 0.034 & 0.238 & 0.133 & 0.065 & 0.161 & 0.116\end{array}$ $\begin{array}{llllllll}41.32 & 0.017 & 0.238 & 0.118 & 0.096 & 0.114 & 0.168\end{array}$ $\begin{array}{llllllll}41.45 & 0.017 & 0.227 & 0.084 & 0.093 & 0.155 & 0.171\end{array}$ $\begin{array}{llllllll}42.21 & 0.023 & 0.226 & 0.105 & 0.088 & 0.100 & 0.155\end{array}$ $\begin{array}{llllllll}41.13 & 0.020 & 0.253 & 0.094 & 0.085 & 0.137 & 0.179\end{array}$ $\begin{array}{llllllll}41.75 & 0.014 & 0.230 & 0.095 & 0.087 & 0.137 & 0.179\end{array}$ $\begin{array}{lllllll}42.26 & 0.020 & 0.251 & 0.086 & 0.083 & 0.181 & 0.185\end{array}$ $\begin{array}{llllllll}42.05 & 0.022 & 0.219 & 0.126 & 0.095 & 0.109 & 0.137\end{array}$ $\begin{array}{llllllll}42.03 & 0.017 & 0.223 & 0.081 & 0.100 & 0.128 & 0.128\end{array}$ $\begin{array}{llllllll}41.55 & 0.019 & 0.227 & 0.090 & 0.098 & 0.120 & 0.138\end{array}$ $\begin{array}{llllllll}41.94 & 0.018 & 0.220 & 0.088 & 0.090 & 0.127 & 0.165\end{array}$ $\begin{array}{llllllll}42.10 & 0.020 & 0.213 & 0.101 & 0.094 & 0.182 & 0.218\end{array}$ $\begin{array}{llllllll}41.82 & 0.016 & 0.216 & 0.091 & 0.097 & 0.150 & 0.151\end{array}$ $\begin{array}{llllllll}42.12 & 0.017 & 0.217 & 0.096 & 0.111 & 0.127 & 0.195\end{array}$ $\begin{array}{llllllll}41.89 & 0.014 & 0.228 & 0.076 & 0.109 & 0.141 & 0.194\end{array}$ $\begin{array}{llllllll}4.10 & 0.016 & 0.207 & 0.089 & 0.114 & 0.110 & 0.205\end{array}$ $\begin{array}{lllllll}41.36 & 0.014 & 0.226 & 0.082 & 0.091 & 0.135 & 0.136\end{array}$ $\begin{array}{lllllll}41.23 & 0.019 & 0.228 & 0.118 & 0.099 & 0.163 & 0.187\end{array}$

$\begin{array}{lllllll}42.19 & 0.153 & 0.619 & 0.892 & 0.669 & 0.114 & 0.441\end{array}$ $\begin{array}{llllllll}42.49 & 0.153 & 0.619 & 0.892 & 0.669 & 0.114 & 0.441\end{array}$ $\begin{array}{llllllll}42.49 & 0.153 & 0.619 & 0.892 & 0.669 & 0.114 & 0.441\end{array}$ $\begin{array}{lllllll}42.30 & 0.153 & 0.619 & 0.892 & 0.669 & 0.114 & 0.441\end{array}$ $\begin{array}{llllllll}42.28 & 0.153 & 0.619 & 0.892 & 0.669 & 0.114 & 0.441\end{array}$ $\begin{array}{llllllll}42.49 & 0.198 & 0.534 & 0.714 & 0.305 & 0.138 & 0.373\end{array}$ $\begin{array}{llllllll}42.97 & 0.198 & 0.515 & 0.734 & 0.300 & 0.129 & 0.367\end{array}$ $\begin{array}{llllllll}42.78 & 0.197 & 0.515 & 0.717 & 0.300 & 0.164 & 0.364\end{array}$ $\begin{array}{llllllll}42.12 & 0.155 & 0.549 & 0.805 & 0.493 & 0.147 & 0.454\end{array}$ $\begin{array}{llllllll}42.94 & 0.157 & 0.568 & 0.798 & 0.505 & 0.123 & 0.423\end{array}$ $\begin{array}{llllllll}42.14 & 0.155 & 0.551 & 0.818 & 0.504 & 0.108 & 0.426\end{array}$ $\begin{array}{llllllll}42.49 & 0.142 & 0.606 & 0.857 & 0.656 & 0.117 & 0.421\end{array}$ $\begin{array}{llllllll}42.71 & 0.143 & 0.639 & 0.900 & 0.664 & 0.110 & 0.439\end{array}$ $\begin{array}{llllllll}42.21 & 0.148 & 0.627 & 0.863 & 0.662 & 0.134 & 0.435\end{array}$ $\begin{array}{llllllll}4.57 & 0.065 & 0.840 & 0.201 & 0.153 & 0.219 & 0.219\end{array}$ $\begin{array}{llllllll}42.07 & 0.068 & 0.858 & 0.182 & 0.152 & 0.267 & 0.230\end{array}$ $\begin{array}{lllllll}42.16 & 0.073 & 0.866 & 0.209 & 0.155 & 0.244 & 0.214\end{array}$ $\begin{array}{llllllll}42.42 & 0.097 & 0.767 & 0.479 & 0.413 & 0.194 & 0.263\end{array}$
00.6

00.0

99.7

100.2

100.2

98.8

99.2

99.

99.3

97.9

99.4

99.3

98.5

99.7

99.6

99.4

99.4

99.

99.8

00.0

00.0

99.0

100.6

100.9

101.6

101.1

101.0

101.3

101.1

100.6

101.5

100.6

101.0

100.3

101.1

101.4

101.4 
60438.11 $\begin{array}{lll}605 & 37.77\end{array}$ $\begin{array}{lll}606 & 37.36\end{array}$ $607 \quad 37.32$ $608 \quad 37.41$ 60937.62 61037.50 $\begin{array}{ll}610 & 37.50\end{array}$ $\begin{array}{lll}611 & 37.79\end{array}$ $\begin{array}{ll}612 & 37.27\end{array}$ $613 \quad 37.42$ 61437.23 $\begin{array}{lll}615 & 38.67\end{array}$ $\begin{array}{lll}616 & 38.77\end{array}$ $617 \quad 38.32$ $618 \quad 36.96$ $\begin{array}{lll}620 & 36.57\end{array}$ $\begin{array}{lll}621 & 37.54\end{array}$ 62237.65 $623 \quad 37.68$ 62437.16 $\begin{array}{lll}625 & 37.33\end{array}$ $\begin{array}{ll}626 & 37.28\end{array}$ 62738.60 $\begin{array}{lll}28 & 38.78\end{array}$ $\begin{array}{lll}629 & 38.28\end{array}$ 37.43 Vasagård

63137.78 63238.14 $\begin{array}{lll}633 & 37.94\end{array}$ $\begin{array}{ll}634 & 37.92\end{array}$ $635 \quad 37.76$ $\begin{array}{lll}636 & 38.15 \\ 637 & 39.02\end{array}$ $\begin{array}{lll}637 & 39.02\end{array}$ $\begin{array}{lll}638 & 38.94\end{array}$ $639 \quad 39.15$ $640 \quad 37.88$ 64137.66 $642 \quad 37.69$ $643 \quad 0.00$
6.0 .00 64450.00 $\begin{array}{ll}645 & 0.00\end{array}$ $\begin{array}{ll}646 & 37.97\end{array}$ $647 \quad 38.13$ $\begin{array}{lll}648 & 38.04\end{array}$ 64938.02 $\begin{array}{lll}650 & 38.18\end{array}$ 65137.90 $\begin{array}{llllllll}3.53 & 18.56 & 0.060 & 0.770 & 0.395 & 0.305 & 0.134 & 0.187\end{array}$ $\begin{array}{llllllll}3.70 & 18.33 & 0.061 & 0.780 & 0.388 & 0.317 & 0.143 & 0.195\end{array}$ $\begin{array}{lllllllll}3.64 & 18.32 & 0.096 & 0.611 & 0.731 & 0.540 & 0.097 & 0.319\end{array}$ $\begin{array}{lllllllll}3.44 & 18.49 & 0.089 & 0.616 & 0.682 & 0.524 & 0.097 & 0.324\end{array}$ $\begin{array}{lllllllll}3.79 & 18.59 & 0.087 & 0.616 & 0.713 & 0.531 & 0.104 & 0.333\end{array}$ $\begin{array}{lllllllll}3.73 & 18.41 & 0.119 & 0.401 & 0.620 & 0.243 & 0.133 & 0.328\end{array}$ $\begin{array}{lllllllll}3.64 & 18.75 & 0.122 & 0.409 & 0.650 & 0.250 & 0.139 & 0.348\end{array}$ $\begin{array}{lllllllll}3.60 & 18.70 & 0.120 & 0.415 & 0.631 & 0.243 & 0.145 & 0.334\end{array}$ $\begin{array}{llllllll}3.64 & 18.39 & 0.122 & 0.411 & 0.612 & 0.244 & 0.151 & 0.338\end{array}$ $\begin{array}{llllllll}3.52 & 18.90 & 0.121 & 0.409 & 0.610 & 0.241 & 0.134 & 0.333\end{array}$ $\begin{array}{lllllllll}3.58 & 18.53 & 0.121 & 0.419 & 0.635 & 0.233 & 0.128 & 0.320\end{array}$ $\begin{array}{lllllllll}3.48 & 18.71 & 0.059 & 0.426 & 0.168 & 0.122 & 0.432 & 0.124\end{array}$ $\begin{array}{lllllllll}3.57 & 18.43 & 0.048 & 0.462 & 0.084 & 0.128 & 0.000 & 0.128\end{array}$ $\begin{array}{lllllllll}3.49 & 18.40 & 0.060 & 0.463 & 0.160 & 0.127 & 0.295 & 0.136\end{array}$ $\begin{array}{lllllllll}3.40 & 18.31 & 0.095 & 0.608 & 0.712 & 0.517 & 0.125 & 0.375\end{array}$ $\begin{array}{lllllllll}3.54 & 18.68 & 0.091 & 0.611 & 0.693 & 0.530 & 0.106 & 0.388\end{array}$ $\begin{array}{lllllllll}3.70 & 18.24 & 0.089 & 0.600 & 0.727 & 0.517 & 0.094 & 0.359\end{array}$ $\begin{array}{llllllll}3.50 & 18.59 & 0.065 & 0.817 & 0.422 & 0.374 & 0.114 & 0.172\end{array}$ $\begin{array}{llllllll}3.63 & 18.56 & 0.063 & 0.810 & 0.449 & 0.373 & 0.136 & 0.172\end{array}$ $\begin{array}{llllllll}3.61 & 18.39 & 0.062 & 0.818 & 0.414 & 0.379 & 0.133 & 0.188\end{array}$ $\begin{array}{lllllllll}3.57 & 18.65 & 0.091 & 0.614 & 0.718 & 0.521 & 0.106 & 0.350\end{array}$ $\begin{array}{lllllllll}3.58 & 18.82 & 0.089 & 0.615 & 0.675 & 0.509 & 0.097 & 0.339\end{array}$ $\begin{array}{lllllllll}3.56 & 18.53 & 0.089 & 0.621 & 0.730 & 0.519 & 0.091 & 0.347\end{array}$ $\begin{array}{lllllllll}3.46 & 18.78 & 0.051 & 0.737 & 0.158 & 0.091 & 0.236 & 0.157\end{array}$ $\begin{array}{llllllll}3.60 & 18.35 & 0.050 & 0.739 & 0.150 & 0.088 & 0.178 & 0.156\end{array}$ $\begin{array}{lllllllll}3.37 & 18.44 & 0.049 & 0.716 & 0.157 & 0.093 & 0.201 & 0.155\end{array}$ $\begin{array}{llllllll}3.69 & 18.40 & 0.111 & 0.396 & 0.635 & 0.245 & 0.128 & 0.322\end{array}$ nekulle \#2

$\begin{array}{llllllll}3.60 & 18.47 & 0.066 & 0.813 & 0.415 & 0.402 & 0.111 & 0.180\end{array}$ $\begin{array}{lllllllll}3.68 & 18.76 & 0.064 & 0.820 & 0.440 & 0.391 & 0.124 & 0.182\end{array}$ $\begin{array}{lllllllll}3.51 & 18.46 & 0.063 & 0.812 & 0.421 & 0.400 & 0.133 & 0.182\end{array}$ $\begin{array}{lllllllll}3.51 & 18.56 & 0.066 & 0.818 & 0.451 & 0.366 & 0.123 & 0.172\end{array}$ $\begin{array}{lllllllll}3.55 & 18.22 & 0.067 & 0.823 & 0.424 & 0.367 & 0.119 & 0.199\end{array}$ $\begin{array}{llllllll}3.49 & 18.26 & 0.068 & 0.809 & 0.445 & 0.377 & 0.121 & 0.175\end{array}$ $\begin{array}{llllllll}3.73 & 18.65 & 0.017 & 0.422 & 0.104 & 0.080 & 0.136 & 0.069\end{array}$ $\begin{array}{llllllll}3.71 & 18.60 & 0.016 & 0.422 & 0.120 & 0.086 & 0.124 & 0.080\end{array}$ $\begin{array}{llllllll}3.59 & 18.74 & 0.017 & 0.421 & 0.099 & 0.081 & 0.150 & 0.076\end{array}$ $\begin{array}{llllllll}3.54 & 18.44 & 0.103 & 0.769 & 0.509 & 0.312 & 0.143 & 0.201\end{array}$ $\begin{array}{lllllllll}3.25 & 18.56 & 0.099 & 0.763 & 0.494 & 0.320 & 0.160 & 0.207\end{array}$ $\begin{array}{lllllllll}3.65 & 18.66 & 0.100 & 0.756 & 0.506 & 0.309 & 0.158 & 0.211\end{array}$ $\begin{array}{llllllll}0.00 & 0.00 & 0.000 & 0.000 & 0.000 & 0.003 & 0.005 & 0.000\end{array}$ $\begin{array}{llllllll}0.00 & 0.00 & 0.000 & 0.000 & 0.005 & 0.000 & 0.002 & 0.000\end{array}$ $\begin{array}{lllllllll}0.00 & 0.00 & 0.002 & 0.000 & 0.000 & 0.000 & 0.000 & 0.000\end{array}$ $\begin{array}{llllllll}3.57 & 18.48 & 0.066 & 0.828 & 0.448 & 0.367 & 0.160 & 0.227\end{array}$ $\begin{array}{lllllllll}3.59 & 18.69 & 0.066 & 0.820 & 0.430 & 0.365 & 0.147 & 0.220\end{array}$ $\begin{array}{lllllllll}3.47 & 18.28 & 0.067 & 0.819 & 0.457 & 0.357 & 0.122 & 0.225\end{array}$ $\begin{array}{llllllll}3.49 & 18.57 & 0.087 & 0.717 & 0.436 & 0.286 & 0.138 & 0.184\end{array}$ $\begin{array}{lllllllll}3.65 & 18.79 & 0.088 & 0.711 & 0.465 & 0.281 & 0.137 & 0.181\end{array}$ $\begin{array}{lllllllll}3.61 & 18.61 & 0.088 & 0.729 & 0.435 & 0.286 & 0.144 & 0.197\end{array}$ $\begin{array}{llllllll}42.53 & 0.099 & 0.770 & 0.508 & 0.393 & 0.156 & 0.237\end{array}$ $\begin{array}{lllllll}42.00 & 0.100 & 0.780 & 0.499 & 0.409 & 0.168 & 0.247\end{array}$ $\begin{array}{llllllll}41.98 & 0.159 & 0.611 & 0.941 & 0.697 & 0.114 & 0.405\end{array}$ $\begin{array}{llllllll}42.37 & 0.147 & 0.616 & 0.877 & 0.676 & 0.114 & 0.412\end{array}$ $\begin{array}{llllllll}42.60 & 0.144 & 0.616 & 0.917 & 0.686 & 0.122 & 0.422\end{array}$ $\begin{array}{llllllll}42.19 & 0.198 & 0.401 & 0.798 & 0.313 & 0.156 & 0.416\end{array}$ $\begin{array}{lllll}42.97 & 0.201 & 0.401 & 0.798\end{array}$ $\begin{array}{llll}0.322 & 0.163 & 0.442\end{array}$ $\begin{array}{llllllll}42.85 & 0.199 & 0.415 & 0.812 & 0.313 & 0.170 & 0.425\end{array}$ $\begin{array}{llllllll}42.14 & 0.203 & 0.411 & 0.788 & 0.315 & 0.177 & 0.429\end{array}$ $\begin{array}{lllllll}43.31 & 0.201 & 0.409 & 0.784 & 0.311 & 0.157 & 0.423\end{array}$ $\begin{array}{llllllll}42.46 & 0.201 & 0.419 & 0.816 & 0.301 & 0.150 & 0.406\end{array}$ $\begin{array}{llllllll}42.88 & 0.097 & 0.426 & 0.216 & 0.158 & 0.506 & 0.157\end{array}$ $\begin{array}{llllllll}42.23 & 0.080 & 0.462 & 0.108 & 0.165 & 0.000 & 0.163\end{array}$ $\begin{array}{llllllll}42.16 & 0.099 & 0.463 & 0.206 & 0.164 & 0.346 & 0.172\end{array}$ $\begin{array}{llllllll}41.96 & 0.157 & 0.608 & 0.917 & 0.668 & 0.146 & 0.476\end{array}$ $\begin{array}{lllllllll}4.91 & 0.157 & 0.608 & 0.917 & 0.668 & 0.146 & 0.476\end{array}$ $\begin{array}{llllllll}42.81 & 0.150 & 0.611 & 0.892 & 0.684 & 0.124 & 0.493\end{array}$ $\begin{array}{llllllll}41.80 & 0.147 & 0.600 & 0.936 & 0.667 & 0.110 & 0.456\end{array}$ $\begin{array}{llllllll}42.60 & 0.108 & 0.817 & 0.543 & 0.483 & 0.134 & 0.219\end{array}$ $\begin{array}{llllllll}42.53 & 0.105 & 0.810 & 0.578 & 0.482 & 0.160 & 0.218\end{array}$ $\begin{array}{lllllll}42.14 & 0.103 & 0.818 & 0.533 & 0.489 & 0.156 & 0.238\end{array}$ $\begin{array}{llllllll}42.74 & 0.150 & 0.614 & 0.923 & 0.672 & 0.124 & 0.444\end{array}$ $\begin{array}{llllllll}43.13 & 0.147 & 0.615 & 0.868 & 0.657 & 0.114 & 0.430\end{array}$ $\begin{array}{llllllll}42.46 & 0.148 & 0.621 & 0.939 & 0.670 & 0.106 & 0.440\end{array}$ $\begin{array}{llllllll}43.04 & 0.084 & 0.737 & 0.203 & 0.118 & 0.276 & 0.200\end{array}$ $\begin{array}{llllllll}42.05 & 0.082 & 0.739 & 0.193 & 0.114 & 0.208 & 0.198\end{array}$ $\begin{array}{lllllllll}42.05 & 0.082 & 0.739 & 0.193 & 0.114 & 0.208 & 0.198\end{array}$ $\begin{array}{lllllll}42.16 & 0.185 & 0.396 & 0.817 & 0.316 & 0.150 & 0.408\end{array}$ $\begin{array}{lllllll}42.33 & 0.109 & 0.813 & 0.533 & 0.519 & 0.130 & 0.228\end{array}$ $\begin{array}{llllllll}42.99 & 0.105 & 0.820 & 0.567 & 0.505 & 0.145 & 0.230\end{array}$ $\begin{array}{llllllll}42.30 & 0.104 & 0.812 & 0.541 & 0.517 & 0.156 & 0.231\end{array}$ $\begin{array}{lllllll}42.53 & 0.109 & 0.818 & 0.581 & 0.472 & 0.144 & 0.218\end{array}$ $\begin{array}{llllllll}41.75 & 0.110 & 0.823 & 0.546 & 0.473 & 0.139 & 0.253\end{array}$ $\begin{array}{lllllll}41.84 & 0.113 & 0.809 & 0.573 & 0.487 & 0.142 & 0.222\end{array}$ $\begin{array}{llllllll}42.74 & 0.027 & 0.422 & 0.133 & 0.103 & 0.159 & 0.088\end{array}$ $\begin{array}{llllllll}42.62 & 0.027 & 0.422 & 0.155 & 0.111 & 0.146 & 0.101\end{array}$ $\begin{array}{llllllll}42.94 & 0.028 & 0.421 & 0.128 & 0.105 & 0.176 & 0.096\end{array}$ $\begin{array}{lllllll}42.26 & 0.171 & 0.769 & 0.655 & 0.403 & 0.167 & 0.255\end{array}$ $\begin{array}{lllllll}42.53 & 0.163 & 0.763 & 0.636 & 0.413 & 0.187 & 0.262\end{array}$ $\begin{array}{llllllll}42.76 & 0.165 & 0.756 & 0.652 & 0.398 & 0.185 & 0.268\end{array}$ $\begin{array}{llllllll}0.01 & 0.000 & 0.000 & 0.000 & 0.003 & 0.005 & 0.000\end{array}$ $\begin{array}{llllllll}0.00 & 0.000 & 0.000 & 0.006 & 0.000 & 0.002 & 0.000\end{array}$ $\begin{array}{llll}0.00 & 0.0003 & 0.000 & 0.000\end{array}$ $\begin{array}{llllllllll}0.00 & 0.003 & 0.000 & 0.000 & 0.000 & 0.000 & 0.000\end{array}$ $\begin{array}{llllllll}42.35 & 0.109 & 0.828 & 0.576 & 0.473 & 0.187 & 0.288\end{array}$ $\begin{array}{llllllll}42.83 & 0.109 & 0.820 & 0.553 & 0.471 & 0.173 & 0.280\end{array}$ $\begin{array}{lllllll}41.89 & 0.112 & 0.819 & 0.588 & 0.461 & 0.143 & 0.285\end{array}$ $\begin{array}{llllllll}42.55 & 0.144 & 0.717 & 0.560 & 0.370 & 0.162 & 0.233\end{array}$ $\begin{array}{llllllll}43.06 & 0.145 & 0.711 & 0.599 & 0.363 & 0.161 & 0.229\end{array}$ $\begin{array}{lllllll}42.65 & 0.146 & 0.729 & 0.560 & 0.369 & 0.169 & 0.250\end{array}$
101.6

100.8

100.9

100.9

101.7

100.9

101.5

101.7

100.3

101.5

102.1

101.1

100.8

100.1

100.5

99.6

101.0

101.2

100.8

101.3

101.8

102.2

102.2

101.5

100.5

101.1

102.4

101.3

101.5

100.5

101.1

102.0
101.8

102.3

101.2

100.9

101.6

0.0

0.0

101.5

102.2

101.0

101.5

102.4

101.5 
$652 \quad 37.77$ $\begin{array}{lll}653 & 38.18\end{array}$ 65438.20 $\begin{array}{lll}655 & 38.13\end{array}$ $\begin{array}{lll}656 & 37.81\end{array}$ 65738.08 $658 \quad 37.95$ $\begin{array}{ll}659 & 37.95 \\ 6\end{array}$ 37.74 $\begin{array}{ll}661 & 38.39\end{array}$ 66238.00 66338.01 66437.35 $\begin{array}{ll}665 & 37.49\end{array}$ $666 \quad 37.76$ 66738.36 $\begin{array}{lll}688 & 37.99\end{array}$ $\begin{array}{lll}669 & 38.02\end{array}$ $\begin{array}{lll}670 & 37.41\end{array}$ 67137.64 $672 \quad 37.68$ $673 \quad 38.24$ $\begin{array}{lll}674 & 38.37\end{array}$ $\begin{array}{lll}675 & 38.47\end{array}$ $\begin{array}{lll}677 & 38.11\end{array}$ $\begin{array}{lll}678 & 37.71\end{array}$ $\begin{array}{lll}679 & 37.78\end{array}$ 68038.01 $681 \quad 38.19$ $\begin{array}{ll}682 & 38.20\end{array}$ $683 \quad 37.74$ $684 \quad 38.05$ $\begin{array}{lll}686 & 37.75\end{array}$ $\begin{array}{ll}688 & 38.47\end{array}$ $\begin{array}{lll}689 & 37.77\end{array}$ 69038.36 Vasagå 69137.07 $692 \quad 36.99$ $693 \quad 37.25$ $694 \quad 35.13$ $695 \quad 36.86$ $\begin{array}{lll}696 & 35.99\end{array}$ $697 \quad 37.33$ $\begin{array}{lll}698 & 37.33\end{array}$ $699 \quad 37.33$ $\begin{array}{llllllll}3.51 & 18.67 & 0.093 & 0.702 & 0.453 & 0.251 & 0.152 & 0.181\end{array}$ $\begin{array}{llllllll}3.32 & 18.23 & 0.089 & 0.696 & 0.417 & 0.252 & 0.156 & 0.167\end{array}$ $\begin{array}{llllllll}3.49 & 18.52 & 0.087 & 0.688 & 0.440 & 0.255 & 0.160 & 0.192\end{array}$ $\begin{array}{lllllllll}3.64 & 18.38 & 0.066 & 0.842 & 0.418 & 0.335 & 0.110 & 0.195\end{array}$ $\begin{array}{llllllll}3.68 & 18.50 & 0.066 & 0.825 & 0.458 & 0.333 & 0.143 & 0.202\end{array}$ $\begin{array}{lllllllll}3.50 & 18.72 & 0.065 & 0.835 & 0.440 & 0.327 & 0.142 & 0.178\end{array}$ $\begin{array}{lllllllll}3.63 & 18.39 & 0.059 & 0.300 & 0.519 & 0.232 & 0.117 & 0.145\end{array}$ $\begin{array}{llllllll}3.41 & 18.35 & 0.058 & 0.304 & 0.485 & 0.224 & 0.110 & 0.141\end{array}$ $\begin{array}{llllllll}3.63 & 18.42 & 0.058 & 0.296 & 0.521 & 0.230 & 0.123 & 0.134\end{array}$ $\begin{array}{llllllll}3.60 & 18.58 & 0.065 & 0.257 & 0.539 & 0.210 & 0.144 & 0.131\end{array}$ $\begin{array}{llllllll}3.57 & 18.40 & 0.069 & 0.242 & 0.558 & 0.214 & 0.120 & 0.098\end{array}$ $\begin{array}{lllllllll}3.49 & 18.37 & 0.071 & 0.232 & 0.516 & 0.217 & 0.130 & 0.115\end{array}$ $\begin{array}{lllllllll}3.51 & 18.42 & 0.068 & 0.798 & 0.455 & 0.369 & 0.137 & 0.207\end{array}$ $\begin{array}{lllllllll}3.46 & 18.41 & 0.067 & 0.789 & 0.428 & 0.364 & 0.138 & 0.203\end{array}$ $\begin{array}{llllllll}3.68 & 18.46 & 0.068 & 0.802 & 0.443 & 0.374 & 0.136 & 0.195\end{array}$ $\begin{array}{lllllllll}3.49 & 18.12 & 0.091 & 0.500 & 0.239 & 0.107 & 0.263 & 0.226\end{array}$ $\begin{array}{lllllllll}3.60 & 18.15 & 0.094 & 0.479 & 0.247 & 0.104 & 0.226 & 0.225\end{array}$ $\begin{array}{llllllll}3.52 & 18.27 & 0.090 & 0.513 & 0.226 & 0.098 & 0.253 & 0.264\end{array}$ $\begin{array}{llllllll}3.50 & 18.29 & 0.065 & 0.808 & 0.450 & 0.354 & 0.137 & 0.205\end{array}$ $\begin{array}{lllllllll}3.65 & 18.42 & 0.068 & 0.802 & 0.427 & 0.347 & 0.146 & 0.212\end{array}$ $\begin{array}{llllllll}3.67 & 18.26 & 0.067 & 0.802 & 0.438 & 0.358 & 0.143 & 0.200\end{array}$ $\begin{array}{lllllllll}3.43 & 18.40 & 0.078 & 1.318 & 0.226 & 0.100 & 0.182 & 0.086\end{array}$ $\begin{array}{lllllllll}3.55 & 18.49 & 0.080 & 1.320 & 0.219 & 0.096 & 0.167 & 0.093\end{array}$ $\begin{array}{lllllllll}3.61 & 18.56 & 0.079 & 1.328 & 0.208 & 0.100 & 0.194 & 0.100\end{array}$ $\begin{array}{lllllllll}3.59 & 18.60 & 0.069 & 0.817 & 0.450 & 0.341 & 0.114 & 0.166\end{array}$ $\begin{array}{lllllllll}3.62 & 18.43 & 0.071 & 0.851 & 0.435 & 0.344 & 0.131 & 0.209\end{array}$ $\begin{array}{llllllll}3.70 & 18.34 & 0.067 & 0.831 & 0.463 & 0.346 & 0.134 & 0.195\end{array}$ $\begin{array}{llllllll}3.59 & 18.76 & 0.058 & 0.292 & 0.504 & 0.238 & 0.123 & 0.144\end{array}$ $\begin{array}{llllllll}3.64 & 18.32 & 0.057 & 0.285 & 0.515 & 0.231 & 0.097 & 0.139\end{array}$ $\begin{array}{lllllllll}3.58 & 18.65 & 0.058 & 0.284 & 0.493 & 0.231 & 0.109 & 0.141\end{array}$ $\begin{array}{lllllllll}3.47 & 18.53 & 0.068 & 0.820 & 0.458 & 0.363 & 0.105 & 0.154\end{array}$ $\begin{array}{lllllllll}3.63 & 18.60 & 0.066 & 0.805 & 0.409 & 0.352 & 0.116 & 0.177\end{array}$ $\begin{array}{llllllll}3.50 & 18.71 & 0.064 & 0.824 & 0.447 & 0.351 & 0.123 & 0.169\end{array}$ $\begin{array}{lllllllll}3.52 & 18.40 & 0.067 & 0.835 & 0.416 & 0.343 & 0.130 & 0.184\end{array}$ $\begin{array}{lllllllll}3.57 & 18.61 & 0.066 & 0.840 & 0.448 & 0.355 & 0.102 & 0.172\end{array}$ $\begin{array}{llllllll}3.50 & 18.49 & 0.064 & 0.811 & 0.438 & 0.353 & 0.128 & 0.176\end{array}$ $\begin{array}{llllllll}3.48 & 18.36 & 0.061 & 0.251 & 0.542 & 0.234 & 0.113 & 0.124\end{array}$ $\begin{array}{llllllll}3.72 & 18.58 & 0.060 & 0.251 & 0.529 & 0.235 & 0.066 & 0.139\end{array}$ $\begin{array}{llllllll}3.51 & 18.44 & 0.063 & 0.253 & 0.533 & 0.230 & 0.096 & 0.125\end{array}$ nekulle \#3

$\begin{array}{lllllllll}3.59 & 18.61 & 0.084 & 0.567 & 0.656 & 0.494 & 0.102 & 0.361\end{array}$ $\begin{array}{lllllllll}3.51 & 18.53 & 0.085 & 0.566 & 0.688 & 0.505 & 0.107 & 0.389\end{array}$ $\begin{array}{lllllllll}3.40 & 18.22 & 0.082 & 0.549 & 0.657 & 0.498 & 0.095 & 0.325\end{array}$ $\begin{array}{lllllllll}3.33 & 16.82 & 0.091 & 0.664 & 0.766 & 0.539 & 0.168 & 0.561\end{array}$ $\begin{array}{llllllll}3.40 & 18.34 & 0.089 & 0.651 & 0.704 & 0.522 & 0.119 & 0.397\end{array}$ $\begin{array}{llllllll}3.43 & 17.53 & 0.089 & 0.690 & 0.797 & 0.545 & 0.167 & 0.656\end{array}$ $\begin{array}{lllllllll}3.39 & 18.52 & 0.089 & 0.627 & 0.693 & 0.536 & 0.097 & 0.313\end{array}$ $\begin{array}{lllllllll}3.51 & 18.48 & 0.086 & 0.642 & 0.718 & 0.527 & 0.100 & 0.314 \\ 3.68 & 18.23 & 0.091 & 0.658 & 0.713 & 0.524 & 0.100 & 0.328\end{array}$ $\begin{array}{lllll}42.78 & 0.153 & 0.702 & 0.582\end{array}$

$\begin{array}{llll}4.78 & 0.148 & 0.696 & 0.536\end{array}$

$\begin{array}{llll}42.44 & 0.145 & 0.688 & 0.566\end{array}$

$\begin{array}{llll}42.12 & 0.109 & 0.842 & 0.538\end{array}$

$\begin{array}{llll}42.39 & 0.110 & 0.825 & 0.589\end{array}$

$\begin{array}{llll}42.90 & 0.108 & 0.835 & 0.566\end{array}$

$\begin{array}{lllll}42.14 & 0.098 & 0.300 & 0.667\end{array}$

$\begin{array}{llll}42.05 & 0.097 & 0.304 & 0.625\end{array}$

$\begin{array}{lllll}42.21 & 0.096 & 0.296 & 0.670\end{array}$

$\begin{array}{lllll}42.58 & 0.108 & 0.257 & 0.693\end{array}$

$\begin{array}{llll}42.16 & 0.115 & 0.242 & 0.718\end{array}$

$\begin{array}{lllll}42.10 & 0.118 & 0.232 & 0.664\end{array}$

$\begin{array}{lllll}42.21 & 0.112 & 0.798 & 0.585\end{array}$

$\begin{array}{lllll}42.19 & 0.112 & 0.789 & 0.551\end{array}$

$\begin{array}{lllll}42.30 & 0.113 & 0.802 & 0.570\end{array}$

$\begin{array}{lllll}41.52 & 0.151 & 0.500 & 0.308\end{array}$

$\begin{array}{lllll}41.59 & 0.156 & 0.479 & 0.318\end{array}$

$\begin{array}{lllll}41.87 & 0.149 & 0.513 & 0.291\end{array}$

$\begin{array}{llll}41.91 & 0.108 & 0.808 & 0.579\end{array}$

$\begin{array}{lllll}42.21 & 0.112 & 0.802 & 0.549\end{array}$

$\begin{array}{lllll}41.84 & 0.110 & 0.802 & 0.564\end{array}$

$\begin{array}{lllll}42.16 & 0.129 & 1.318 & 0.291\end{array}$

$\begin{array}{llll}42.37 & 0.132 & 1.320 & 0.282\end{array}$

$\begin{array}{lllll}42.53 & 0.132 & 1.328 & 0.268\end{array}$

$\begin{array}{lllll}42.62 & 0.115 & 0.817 & 0.578\end{array}$

$\begin{array}{lllll}42.23 & 0.117 & 0.851 & 0.560\end{array}$

$\begin{array}{lllll}42.03 & 0.110 & 0.831 & 0.596\end{array}$

$\begin{array}{lllll}42.99 & 0.097 & 0.292 & 0.648\end{array}$

$\begin{array}{lllll}41.98 & 0.094 & 0.285 & 0.662\end{array}$

$\begin{array}{lllll}42.74 & 0.095 & 0.284 & 0.634\end{array}$

$\begin{array}{lllll}42.46 & 0.112 & 0.820 & 0.589\end{array}$

$\begin{array}{lllll}42.62 & 0.109 & 0.805 & 0.527\end{array}$

$\begin{array}{lllll}42.88 & 0.107 & 0.824 & 0.575\end{array}$

$\begin{array}{lllll}42.16 & 0.110 & 0.835 & 0.535\end{array}$

$\begin{array}{lllll}42.65 & 0.110 & 0.840 & 0.577\end{array}$

$\begin{array}{lllll}42.37 & 0.106 & 0.811 & 0.564\end{array}$

$\begin{array}{llll}42.07 & 0.101 & 0.251 & 0.697\end{array}$

$\begin{array}{lllll}42.58 & 0.099 & 0.251 & 0.681\end{array}$

$\begin{array}{lllll}42.26 & 0.105 & 0.253 & 0.686\end{array}$

$\begin{array}{lllll}42.65 & 0.140 & 0.567 & 0.844\end{array}$

$\begin{array}{lllll}42.46 & 0.141 & 0.566 & 0.886\end{array}$

$\begin{array}{lllll}41.75 & 0.136 & 0.549 & 0.845\end{array}$

$\begin{array}{lllll}38.54 & 0.151 & 0.664 & 0.985\end{array}$

$\begin{array}{llll}42.03 & 0.148 & 0.651 & 0.905\end{array}$

$\begin{array}{lllll}40.17 & 0.148 & 0.690 & 1.025\end{array}$

$\begin{array}{lllll}42.44 & 0.147 & 0.627 & 0.891\end{array}$

$\begin{array}{llll}42.35 & 0.142 & 0.642 & 0.924 \\ 41.78 & 0.150 & 0.658 & 0.917\end{array}$ $\begin{array}{lll}0.324 & 0.178 & 0.229\end{array}$

$\begin{array}{llll}0.325 & 0.183 & 0.212\end{array}$

$\begin{array}{llll}0.329 & 0.187 & 0.243\end{array}$

$\begin{array}{llll}0.432 & 0.128 & 0.248\end{array}$

$\begin{array}{lll}0.430 & 0.167 & 0.256\end{array}$

$\begin{array}{llll}0.422 & 0.166 & 0.226\end{array}$

$\begin{array}{llll}0.299 & 0.137 & 0.185\end{array}$

$\begin{array}{llll}0.289 & 0.128 & 0.179\end{array}$

$\begin{array}{lll}0.297 & 0.144 & 0.171\end{array}$

$\begin{array}{lll}0.271 & 0.168 & 0.166\end{array}$

$\begin{array}{lll}0.277 & 0.141 & 0.124\end{array}$

$\begin{array}{llll}0.280 & 0.152 & 0.146\end{array}$

$\begin{array}{lll}.477 & 0.161 & 0.263\end{array}$

$\begin{array}{llll}0.483 & 0.159 & 0.247\end{array}$

$\begin{array}{lll}0.139 & 0.308 & 0.287\end{array}$

$\begin{array}{lll}0.134 & 0.265 & 0.286\end{array}$

$\begin{array}{llll}0.126 & 0.297 & 0.335\end{array}$

$\begin{array}{lll}0.457 & 0.161 & 0.260\end{array}$

$\begin{array}{llll}0.447 & 0.170 & 0.270\end{array}$

$\begin{array}{llll}0.463 & 0.167 & 0.253\end{array}$

$\begin{array}{llll}0.130 & 0.213 & 0.109\end{array}$

$\begin{array}{lll}0.124 & 0.196 & 0.118\end{array}$

$\begin{array}{lll}0.130 & 0.228 & 0.127\end{array}$

$\begin{array}{lll}0.440 & 0.133 & 0.210\end{array}$

$\begin{array}{llll}0.444 & 0.153 & 0.266\end{array}$

$\begin{array}{llll}0.444 & 0.153 & 0.266\end{array}$

$\begin{array}{llll}0.307 & 0.144 & 0.183\end{array}$

$\begin{array}{lll}0.298 & 0.113 & 0.177\end{array}$

$\begin{array}{llll}0.299 & 0.127 & 0.179\end{array}$

$\begin{array}{llll}0.469 & 0.123 & 0.196\end{array}$

$\begin{array}{lll}0.455 & 0.136 & 0.224\end{array}$

$\begin{array}{llll}0.453 & 0.143 & 0.215\end{array}$

$\begin{array}{llll}0.443 & 0.152 & 0.233\end{array}$

$\begin{array}{llll}0.458 & 0.119 & 0.218\end{array}$

$\begin{array}{lll}0.456 & 0.150 & 0.223\end{array}$

$\begin{array}{lll}0.302 & 0.132 & 0.158\end{array}$

$\begin{array}{lll}0.303 & 0.078 & 0.177\end{array}$

$\begin{array}{llll}0.297 & 0.112 & 0.159\end{array}$

$\begin{array}{lll}0.638 & 0.119 & 0.459\end{array}$

$\begin{array}{lll}0.652 & 0.125 & 0.494\end{array}$

$\begin{array}{llll}0.643 & 0.111 & 0.413\end{array}$

$\begin{array}{llll}0.696 & 0.196 & 0.712\end{array}$

$\begin{array}{llll}0.675 & 0.139 & 0.504\end{array}$

$\begin{array}{llll}0.704 & 0.196 & 0.833\end{array}$

$\begin{array}{llll}0.692 & 0.114 & 0.397\end{array}$

$\begin{array}{lll}0.692 & 0.114 & 0.397 \\ 0.681 & 0.118 & 0.398 \\ 0.677 & 0.117 & 0.416\end{array}$

$\begin{array}{llll}0.677 & 0.117 & 0.416\end{array}$
101.3

00.

101.6

01.4

102.0

102.0

(00.6

100.2

101.6

100.6

100.4

100.4

100.5

101.2

100.2

100.9

100.6

101.3

101.8

102.2

100.9

101.1

100.5

101.4

101.7

101.3

101.2

01.4

101.0

101.1

100.8

101.1

100.9

100.6

100.0

94.5

100.1
97.6

101.0

101.0 
70036.98 $\begin{array}{lll}701 & 36.98\end{array}$ $702 \quad 36.79$ $703 \quad 37.92$ $704 \quad 37.96$ $705 \quad 37.81$ $706 \quad 36.80$ $707 \quad 36.83$ $\begin{array}{lll}708 & 37.17\end{array}$ $\begin{array}{lll}709 & 38.22\end{array}$ $710 \quad 38.33$ $\begin{array}{lll}711 & 37.79\end{array}$ $712 \quad 36.79$ $713 \quad 36.73$ $714 \quad 36.22$ $715 \quad 36.74$ $716 \quad 36.57$ $717 \quad 36.76$ $718 \quad 36.63$ $\begin{array}{lll}719 & 36.99\end{array}$ $\begin{array}{lll}720 & 36.51\end{array}$ $\begin{array}{lll}721 & 37.17\end{array}$ $\begin{array}{lll}722 & 37.07\end{array}$ $723 \quad 37.10$ 72436.85 $\begin{array}{lll}725 & 36.88\end{array}$ $\begin{array}{lll}726 & 36.92\end{array}$ $\begin{array}{lll}727 & 37.21\end{array}$ $728 \quad 37.34$ 72937.15 $\begin{array}{lll}730 & 37.15\end{array}$ 73137.11 $732 \quad 36.96$ 73336.65 $734 \quad 36.60$ $\begin{array}{lll}735 & 36.57\end{array}$ $736 \quad 36.76$ $\begin{array}{lll}737 & 36.47\end{array}$ $\begin{array}{lll}738 & 36.87\end{array}$ $\begin{array}{lll}739 & 36.77\end{array}$ $740 \quad 36.53$ $741 \quad 36.92$ $\begin{array}{lll}741 & 36.92\end{array}$ $\begin{array}{lll}742 & 35.26\end{array}$ $\begin{array}{lll}743 & 34.57\end{array}$ $\begin{array}{lll}744 & 37.11\end{array}$ $745 \quad 36.86$ $746 \quad 36.71$ $747 \quad 36.76$ $748 \quad 37.56$ $\begin{array}{llllllll}3.26 & 18.06 & 0.088 & 0.659 & 0.739 & 0.551 & 0.131 & 0.437\end{array}$ $\begin{array}{llllllll}3.57 & 18.41 & 0.096 & 0.648 & 0.721 & 0.555 & 0.123 & 0.425\end{array}$ $\begin{array}{llllllll}3.52 & 18.10 & 0.093 & 0.633 & 0.736 & 0.541 & 0.133 & 0.402\end{array}$ $\begin{array}{lllllllll}3.63 & 18.09 & 0.050 & 0.755 & 0.125 & 0.099 & 0.199 & 0.125\end{array}$ $\begin{array}{lllllllll}3.40 & 18.28 & 0.049 & 0.736 & 0.137 & 0.104 & 0.193 & 0.107\end{array}$ $\begin{array}{llllllll}3.58 & 18.22 & 0.050 & 0.750 & 0.138 & 0.095 & 0.193 & 0.123\end{array}$ $\begin{array}{lllllllll}3.48 & 18.19 & 0.077 & 0.599 & 0.634 & 0.498 & 0.093 & 0.340\end{array}$ $\begin{array}{lllllllll}3.50 & 18.04 & 0.078 & 0.617 & 0.620 & 0.493 & 0.096 & 0.330\end{array}$ $\begin{array}{lllllllll}3.58 & 18.11 & 0.079 & 0.611 & 0.647 & 0.496 & 0.100 & 0.330\end{array}$ $\begin{array}{llllllll}3.55 & 18.57 & 0.049 & 0.763 & 0.139 & 0.105 & 0.210 & 0.115\end{array}$ $\begin{array}{lllllllll}3.34 & 18.24 & 0.051 & 0.786 & 0.133 & 0.106 & 0.202 & 0.102\end{array}$ $\begin{array}{lllllllll}3.56 & 18.34 & 0.051 & 0.759 & 0.130 & 0.107 & 0.224 & 0.108\end{array}$ $\begin{array}{lllllllll}3.56 & 18.08 & 0.086 & 0.639 & 0.679 & 0.516 & 0.122 & 0.390\end{array}$ $\begin{array}{lllllllll}3.52 & 18.26 & 0.083 & 0.626 & 0.675 & 0.517 & 0.120 & 0.409\end{array}$ $\begin{array}{llllllll}3.46 & 18.27 & 0.083 & 0.635 & 0.689 & 0.513 & 0.109 & 0.389\end{array}$ $\begin{array}{lllllllll}3.44 & 18.00 & 0.086 & 0.617 & 0.648 & 0.501 & 0.112 & 0.367\end{array}$ $\begin{array}{lllllllll}3.42 & 18.41 & 0.083 & 0.636 & 0.670 & 0.504 & 0.107 & 0.365\end{array}$ $\begin{array}{llllllll}3.52 & 18.41 & 0.085 & 0.618 & 0.695 & 0.507 & 0.105 & 0.385\end{array}$ $\begin{array}{lllllllll}3.51 & 18.72 & 0.085 & 0.630 & 0.702 & 0.521 & 0.097 & 0.321\end{array}$ $\begin{array}{lllllllll}3.45 & 18.24 & 0.088 & 0.621 & 0.660 & 0.515 & 0.084 & 0.314\end{array}$ $\begin{array}{lllllllll}3.50 & 18.27 & 0.087 & 0.606 & 0.697 & 0.514 & 0.082 & 0.314\end{array}$ $\begin{array}{lllllllll}3.50 & 18.34 & 0.146 & 0.479 & 0.553 & 0.205 & 0.178 & 0.316\end{array}$ $\begin{array}{lllllllll}3.50 & 17.80 & 0.136 & 0.479 & 0.551 & 0.202 & 0.185 & 0.304\end{array}$ $\begin{array}{llllllll}3.55 & 18.13 & 0.145 & 0.472 & 0.544 & 0.199 & 0.168 & 0.281\end{array}$ $\begin{array}{lllllllll}3.50 & 18.25 & 0.084 & 0.622 & 0.673 & 0.508 & 0.100 & 0.326\end{array}$ $\begin{array}{lllllllll}3.41 & 18.56 & 0.086 & 0.616 & 0.675 & 0.520 & 0.096 & 0.337\end{array}$ $\begin{array}{lllllllll}3.51 & 18.28 & 0.084 & 0.622 & 0.667 & 0.514 & 0.088 & 0.326\end{array}$ $\begin{array}{llllllll}3.40 & 18.39 & 0.089 & 0.488 & 0.675 & 0.530 & 0.118 & 0.371\end{array}$ $\begin{array}{llllllll}3.55 & 18.43 & 0.088 & 0.632 & 0.700 & 0.495 & 0.105 & 0.323\end{array}$ $\begin{array}{lllllllll}3.48 & 18.43 & 0.088 & 0.473 & 0.670 & 0.516 & 0.107 & 0.371\end{array}$ $\begin{array}{lllllllll}3.46 & 18.36 & 0.095 & 0.541 & 0.585 & 0.345 & 0.121 & 0.341\end{array}$ $\begin{array}{lllllllll}3.46 & 18.51 & 0.093 & 0.551 & 0.561 & 0.341 & 0.122 & 0.354\end{array}$ $\begin{array}{lllllllll}3.58 & 18.45 & 0.090 & 0.556 & 0.575 & 0.343 & 0.109 & 0.337\end{array}$ $\begin{array}{llllllll}3.49 & 18.32 & 0.085 & 0.633 & 0.671 & 0.515 & 0.078 & 0.320\end{array}$ $\begin{array}{lllllllll}3.61 & 18.34 & 0.086 & 0.624 & 0.697 & 0.528 & 0.090 & 0.327\end{array}$ $\begin{array}{lllllllll}3.62 & 18.51 & 0.087 & 0.625 & 0.687 & 0.532 & 0.095 & 0.324\end{array}$ $\begin{array}{lllllllll}3.60 & 18.23 & 0.083 & 0.597 & 0.689 & 0.514 & 0.137 & 0.425\end{array}$ $\begin{array}{llllllll}3.52 & 18.21 & 0.087 & 0.590 & 0.661 & 0.509 & 0.111 & 0.399\end{array}$ $\begin{array}{lllllllll}3.51 & 18.51 & 0.085 & 0.588 & 0.669 & 0.504 & 0.121 & 0.329\end{array}$ $\begin{array}{lllllllll}3.62 & 18.26 & 0.090 & 0.517 & 0.689 & 0.525 & 0.118 & 0.357\end{array}$ $\begin{array}{lllllllll}3.58 & 18.17 & 0.092 & 0.514 & 0.707 & 0.516 & 0.098 & 0.342\end{array}$ $\begin{array}{lllllllll}3.60 & 18.65 & 0.090 & 0.519 & 0.690 & 0.528 & 0.122 & 0.354\end{array}$ $\begin{array}{llllllll}3.27 & 18.34 & 0.074 & 0.591 & 0.629 & 0.458 & 0.109 & 0.321\end{array}$ $\begin{array}{llllllll}3.22 & 16.97 & 0.085 & 0.633 & 0.662 & 0.525 & 0.095 & 0.355\end{array}$ $\begin{array}{llllllll}3.31 & 18.66 & 0.087 & 0.635 & 0.696 & 0.522 & 0.113 & 0.353\end{array}$ $\begin{array}{llllllll}3.56 & 17.95 & 0.095 & 0.525 & 0.623 & 0.376 & 0.134 & 0.379\end{array}$ $\begin{array}{lllllllll}3.53 & 18.64 & 0.089 & 0.537 & 0.620 & 0.382 & 0.129 & 0.391\end{array}$ $\begin{array}{lllllllll}3.45 & 17.90 & 0.091 & 0.535 & 0.611 & 0.371 & 0.128 & 0.391\end{array}$ $\begin{array}{lllllllll}3.45 & 18.17 & 0.095 & 1.153 & 0.261 & 0.134 & 0.232 & 0.120\end{array}$
51.77

51.77

3.63

$53.14 \quad 3.40$

$52.93 \quad 3.58$

$51.52 \quad 3.48$

$51.56 \quad 3.50$

$52.04 \quad 3.58$

$53.51 \quad 3.55$

$53.66 \quad 3.34$

$52.91 \quad 3.56$

$51.51 \quad 3.56$

$50.71 \quad 3.46$

$51.44 \quad 3.44$

$51.20 \quad 3.42$

$51.46 \quad 3.52$

$\begin{array}{ll}51.28 & 3.51\end{array}$

$51.79 \quad 3.45$

$51.11 \quad 3.50$

$52.04 \quad 3.50$

$51.94 \quad 3.55$

$51.59 \quad 3.50$

$51.63 \quad 3.41$

$51.69 \quad 3.51$

$52.09 \quad 3.40$

$52.28 \quad 3.55$

$\begin{array}{ll}52.01 & 3.48 \\ 52.01 & 3.46\end{array}$

$51.95 \quad 3.46$

$51.74 \quad 3.58$

$51.31 \quad 3.49$

$51.24 \quad 3.61$

$51.20 \quad 3.62$

$51.46 \quad 3.60$

$51.06 \quad 3.52$

$51.62 \quad 3.51$

$51.48 \quad 3.62$

$\begin{array}{ll}51.14 & 3.58 \\ 51.69 & 3.60\end{array}$

$\begin{array}{ll}48.40 \quad 3.22 \\ 51.95 & 3.31\end{array}$

$51.95 \quad 3.31$

$51.60 \quad 3.56$

$51.39 \quad 3.53$

$\begin{array}{ll}51.46 & 3.45 \\ 52.58 & 3.45\end{array}$ $\begin{array}{llll}41.39 & 0.147 & 0.659 & 0.950\end{array}$

$\begin{array}{lllll}42.19 & 0.159 & 0.648 & 0.928\end{array}$

$\begin{array}{llll}41.48 & 0.155 & 0.633 & 0.948\end{array}$

$\begin{array}{lllll}41.45 & 0.083 & 0.755 & 0.160\end{array}$

$\begin{array}{lllll}41.89 & 0.081 & 0.736 & 0.176\end{array}$

$\begin{array}{lllll}41.75 & 0.083 & 0.750 & 0.177\end{array}$

$\begin{array}{lllll}41.68 & 0.127 & 0.599 & 0.816\end{array}$

$\begin{array}{lllll}41.34 & 0.129 & 0.617 & 0.798\end{array}$

$\begin{array}{lllll}4.30 & 0.129 & 0.617 & 0.798\end{array}$

$\begin{array}{lllll}42.55 & 0.081 & 0.763 & 0.179\end{array}$

$\begin{array}{lllll}1.80 & 0.085 & 0.786 & 0.171\end{array}$

$\begin{array}{lllll}42.03 & 0.084 & 0.759 & 0.167\end{array}$

$\begin{array}{lllll}41.43 & 0.143 & 0.639 & 0.874\end{array}$

$\begin{array}{lllll}41.84 & 0.137 & 0.626 & 0.869\end{array}$

$\begin{array}{lllll}41.87 & 0.137 & 0.635 & 0.887\end{array}$

$\begin{array}{lllll}1.25 & 0.142 & 0.617 & 0.834\end{array}$

$\begin{array}{lllll}42.19 & 0.138 & 0.636 & 0.862\end{array}$

$\begin{array}{lllll}42.19 & 0.141 & 0.618 & 0.894\end{array}$

$\begin{array}{lllll}42.90 & 0.141 & 0.630 & 0.903\end{array}$

$\begin{array}{lllll}41.80 & 0.146 & 0.621 & 0.849\end{array}$

$\begin{array}{lllll}41.87 & 0.144 & 0.606 & 0.897\end{array}$

$\begin{array}{lllll}42.03 & 0.241 & 0.479 & 0.712\end{array}$

$\begin{array}{lllll}40.79 & 0.226 & 0.479 & 0.708\end{array}$

$\begin{array}{lllll}41.55 & 0.240 & 0.472 & 0.699\end{array}$

$\begin{array}{lllll}41.82 & 0.139 & 0.622 & 0.865\end{array}$

$\begin{array}{lllll}42.53 & 0.143 & 0.616 & 0.868\end{array}$

$\begin{array}{lllll}41.89 & 0.133 & 0.616 & 0.868\end{array}$

$\begin{array}{llll}42.14 & 0.148 & 0.488 & 0.868\end{array}$

$\begin{array}{lllll}42.23 & 0.146 & 0.632 & 0.901\end{array}$

$\begin{array}{lllll}42.23 & 0.146 & 0.473 & 0.861\end{array}$

$\begin{array}{lllll}42.07 & 0.157 & 0.541 & 0.753\end{array}$

$\begin{array}{lllll}42.42 & 0.154 & 0.551 & 0.721\end{array}$

$\begin{array}{lllll}42.28 & 0.150 & 0.556 & 0.740\end{array}$

$\begin{array}{lllll}41.98 & 0.141 & 0.633 & 0.863\end{array}$

$\begin{array}{lllll}42.03 & 0.142 & 0.624 & 0.897\end{array}$

$\begin{array}{lllll}42.42 & 0.145 & 0.625 & 0.884\end{array}$

$\begin{array}{llll}41.78 & 0.137 & 0.597 & 0.887\end{array}$

$\begin{array}{lllll}41.73 & 0.144 & 0.590 & 0.850\end{array}$

$\begin{array}{lllll}42.42 & 0.142 & 0.588 & 0.861\end{array}$

$\begin{array}{lllll}41.84 & 0.149 & 0.517 & 0.886\end{array}$

$\begin{array}{lllll}41.64 & 0.152 & 0.514 & 0.910\end{array}$

$\begin{array}{lllll}42.74 & 0.149 & 0.519 & 0.887\end{array}$

$\begin{array}{lllll}42.03 & 0.122 & 0.591 & 0.809\end{array}$

$\begin{array}{lllll}38.89 & 0.141 & 0.633 & 0.851\end{array}$

$\begin{array}{llll}42.76 & 0.145 & 0.635 & 0.895\end{array}$

$\begin{array}{lllll}41.13 & 0.157 & 0.525 & 0.801\end{array}$

$\begin{array}{lllll}42.71 & 0.148 & 0.537 & 0.797\end{array}$

$\begin{array}{lllll}41.02 & 0.151 & 0.535 & 0.786\end{array}$

$\begin{array}{lllll}41.64 & 0.158 & 1.153 & 0.335\end{array}$ $\begin{array}{lll}0.712 & 0.154 & 0.555\end{array}$

$\begin{array}{llll}0.717 & 0.144 & 0.540\end{array}$

$\begin{array}{lll}0.698 & 0.156 & 0.510\end{array}$

$\begin{array}{lll}128 & 0.233 & 0.159\end{array}$

$\begin{array}{lll}0.134 & 0.226 & 0.136\end{array}$

$\begin{array}{llll}0.123 & 0.226 & 0.156\end{array}$

$\begin{array}{llll}0.643 & 0.109 & 0.431\end{array}$

$\begin{array}{lll}0.636 & 0.112 & 0.419\end{array}$

$\begin{array}{llll}0.641 & 0.118 & 0.419\end{array}$

$\begin{array}{lll}0.135 & 0.246 & 0.146\end{array}$

$\begin{array}{lll}0.137 & 0.236 & 0.130\end{array}$

$\begin{array}{lll}0.138 & 0.263 & 0.137\end{array}$

$\begin{array}{lll}066 & 0.143 & 0.495\end{array}$

$\begin{array}{lll}0.667 & 0.140 & 0.519\end{array}$

$\begin{array}{lll}0.662 & 0.128 & 0.494\end{array}$

$\begin{array}{llll}0.647 & 0.131 & 0.466\end{array}$

$\begin{array}{lll}0.651 & 0.126 & 0.463\end{array}$

$\begin{array}{lll}0.654 & 0.122 & 0.489\end{array}$

$\begin{array}{lll}0.673 & 0.114 & 0.408\end{array}$

$\begin{array}{lll}0.665 & 0.098 & 0.399\end{array}$

$\begin{array}{lll}0.663 & 0.096 & 0.399\end{array}$

$\begin{array}{lll}264 & 0.208 & 0.402\end{array}$

$\begin{array}{llll}0.260 & 0.216 & 0.386\end{array}$

$\begin{array}{llll}0.257 & 0.197 & 0.356\end{array}$

$\begin{array}{llll}0.656 & 0.117 & 0.413\end{array}$

$\begin{array}{llll}0.672 & 0.112 & 0.427\end{array}$

$\begin{array}{llll}0.664 & 0.103 & 0.414\end{array}$

$\begin{array}{lll}0.684 & 0.138 & 0.471\end{array}$

$\begin{array}{lll}0.639 & 0.123 & 0.410\end{array}$

$\begin{array}{lll}0.667 & 0.125 & 0.472\end{array}$

$\begin{array}{llll}0.445 & 0.142 & 0.433\end{array}$

$\begin{array}{lll}0.440 & 0.143 & 0.449\end{array}$

$\begin{array}{llll}0.665 & 0.091 & 0.406\end{array}$

$\begin{array}{llll}0.682 & 0.105 & 0.415\end{array}$

$\begin{array}{lll}0.686 & 0.111 & 0.412\end{array}$

$\begin{array}{llll}0.663 & 0.161 & 0.539\end{array}$

$\begin{array}{llll}0.657 & 0.130 & 0.507\end{array}$

$\begin{array}{lll}0.651 & 0.142 & 0.418\end{array}$

$\begin{array}{llll}0.677 & 0.139 & 0.453\end{array}$

$\begin{array}{llll}0.666 & 0.114 & 0.434\end{array}$

$\begin{array}{lll}0.681 & 0.143 & 0.449\end{array}$

$\begin{array}{llll}0.591 & 0.128 & 0.408\end{array}$

$\begin{array}{llll}0.678 & 0.111 & 0.451\end{array}$

$\begin{array}{llll}0.674 & 0.133 & 0.449\end{array}$

$\begin{array}{llll}0.485 & 0.157 & 0.481\end{array}$

$\begin{array}{llll}0.493 & 0.151 & 0.496\end{array}$

$\begin{array}{lll}0.479 & 0.150 & 0.497 \\ 0.173 & 0.272 & 0.153\end{array}$
99.6

00.7

99.6

99.8

99.1

101.2

100.3

100.0

99.5

99.0

99.0

99.7

100.1

00.6

99.3

98.5

100.4

99.9

100.4

100.9

100.5

100.0

100.3

100.0

99.6

99.7

100.1

99.8
99.2

100.3

99.8

99.2

93.4

01.0

98.9

100.3

99.9 
74937.38 \begin{tabular}{l}
$750 \quad 37.53$ \\
\hline
\end{tabular}

$\begin{array}{llllllll}3.54 & 18.01 & 0.096 & 1.172 & 0.249 & 0.132 & 0.230 & 0.123\end{array}$ Vasag

$\begin{array}{lllllllll}3.52 & 18.11 & 0.100 & 1.170 & 0.274 & 0.128 & 0.236 & 0.123\end{array}$ $752 \quad 37.35$

$753 \quad 37.24$

75437.11

$755 \quad 36.85$

$756 \quad 37.01$

\begin{tabular}{l}
$757 \quad 36.99$ \\
\hline
\end{tabular}

$\begin{array}{lll}758 & 36.56\end{array}$

$\begin{array}{lll}759 & 37.23\end{array}$

$\begin{array}{lll}760 & 36.87\end{array}$

$761 \quad 37.12$

76237.00

$763 \quad 36.77$

$\begin{array}{lll}764 & 36.98\end{array}$

$\begin{array}{lll}764 & 36.98 \\ 765 & 37.14\end{array}$

$\begin{array}{lll}766 & 36.99\end{array}$

$767 \quad 36.73$

$\begin{array}{lll}768 & 37.37\end{array}$

$\begin{array}{lll}769 & 37.42\end{array}$

$\begin{array}{lll}770 & 37.02\end{array}$

77137.09

$\begin{array}{lll}772 & 37.18\end{array}$

$\begin{array}{lll}772 & 37.18 \\ 773 & 37.34\end{array}$

77437.23

$\begin{array}{lll}775 & 37.49\end{array}$

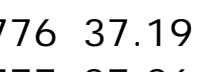

$\begin{array}{lll}777 & 37.26\end{array}$

$\begin{array}{lll}778 & 36.79\end{array}$

$\begin{array}{lll}779 & 37.17\end{array}$

$\begin{array}{lll}780 & 37.18\end{array}$

78137.09

$\begin{array}{lll}781 & 37.09 \\ 782 & 36.90\end{array}$

$\begin{array}{lll}782 & 36.90\end{array}$

$\begin{array}{lll}784 & 37.08\end{array}$

78537.40

$\begin{array}{lll}786 & 37.62\end{array}$

$787 \quad 37.32$

$\begin{array}{lll}788 & 37.08\end{array}$

$\begin{array}{lll}789 & 37.29\end{array}$

$\begin{array}{lll}790 & 37.24\end{array}$

$\begin{array}{lll}790 & 37.24 \\ 791 & 37.02\end{array}$

79237.53

$\begin{array}{lll}793 & 37.31\end{array}$

79436.95

$795 \quad 37.09$

79637.36

$797 \quad 36.85$

$\begin{array}{llllllll}3.53 & 18.46 & 0.106 & 0.546 & 0.570 & 0.291 & 0.125 & 0.327\end{array}$ $\begin{array}{lllllllll}3.57 & 18.47 & 0.106 & 0.552 & 0.565 & 0.295 & 0.113 & 0.309\end{array}$ $\begin{array}{lllllllll}3.52 & 18.35 & 0.106 & 0.555 & 0.561 & 0.296 & 0.130 & 0.337\end{array}$ $\begin{array}{lllllllll}3.61 & 18.35 & 0.089 & 0.641 & 0.712 & 0.518 & 0.108 & 0.348\end{array}$ $\begin{array}{llllllll}3.55 & 18.45 & 0.090 & 0.630 & 0.680 & 0.523 & 0.098 & 0.352\end{array}$ $\begin{array}{llllllll}3.57 & 18.63 & 0.089 & 0.637 & 0.714 & 0.509 & 0.100 & 0.340\end{array}$ $\begin{array}{llllllll}3.49 & 18.64 & 0.087 & 0.624 & 0.692 & 0.509 & 0.105 & 0.382\end{array}$ $\begin{array}{lllllllll}3.51 & 18.05 & 0.082 & 0.634 & 0.664 & 0.499 & 0.124 & 0.388\end{array}$ $\begin{array}{lllllllll}3.43 & 18.68 & 0.086 & 0.626 & 0.670 & 0.497 & 0.129 & 0.376\end{array}$ $\begin{array}{lllllllll}3.57 & 18.44 & 0.084 & 0.616 & 0.676 & 0.509 & 0.103 & 0.329\end{array}$ $\begin{array}{lllllllll}3.71 & 18.21 & 0.085 & 0.627 & 0.662 & 0.506 & 0.104 & 0.356\end{array}$ $\begin{array}{lllllllll}3.50 & 18.28 & 0.083 & 0.618 & 0.671 & 0.498 & 0.090 & 0.328\end{array}$ $\begin{array}{lllllllll}3.54 & 18.35 & 0.087 & 0.617 & 0.625 & 0.496 & 0.112 & 0.323\end{array}$ $\begin{array}{lllllllll}3.69 & 18.23 & 0.081 & 0.611 & 0.651 & 0.493 & 0.107 & 0.309\end{array}$ $\begin{array}{llllllll}3.44 & 18.45 & 0.083 & 0.626 & 0.642 & 0.499 & 0.089 & 0.321\end{array}$ $\begin{array}{lllllllll}3.47 & 18.43 & 0.086 & 0.628 & 0.668 & 0.499 & 0.096 & 0.348\end{array}$ $\begin{array}{lllllllll}3.61 & 18.50 & 0.087 & 0.637 & 0.660 & 0.492 & 0.101 & 0.342\end{array}$ $\begin{array}{lllllllll}3.49 & 18.47 & 0.087 & 0.611 & 0.696 & 0.500 & 0.094 & 0.362\end{array}$ $\begin{array}{lllllllll}3.66 & 18.47 & 0.091 & 0.544 & 0.626 & 0.396 & 0.102 & 0.358\end{array}$ $\begin{array}{lllllllll}3.53 & 18.19 & 0.087 & 0.538 & 0.628 & 0.397 & 0.083 & 0.336\end{array}$ $\begin{array}{lllllllll}3.52 & 18.89 & 0.091 & 0.548 & 0.622 & 0.403 & 0.090 & 0.338\end{array}$ $\begin{array}{lllllllll}3.58 & 18.24 & 0.119 & 0.438 & 0.634 & 0.248 & 0.143 & 0.341\end{array}$ $\begin{array}{llllllll}3.62 & 18.26 & 0.117 & 0.425 & 0.629 & 0.241 & 0.143 & 0.325\end{array}$ $\begin{array}{llllllll}3.45 & 18.41 & 0.085 & 0.625 & 0.672 & 0.506 & 0.109 & 0.361\end{array}$ $\begin{array}{llllllll}3.40 & 18.48 & 0.087 & 0.651 & 0.745 & 0.501 & 0.118 & 0.408\end{array}$ $\begin{array}{lllllllll}3.60 & 18.10 & 0.088 & 0.654 & 0.676 & 0.509 & 0.098 & 0.348\end{array}$ $\begin{array}{lllllllll}3.46 & 18.39 & 0.078 & 0.608 & 0.646 & 0.490 & 0.104 & 0.324\end{array}$ $\begin{array}{llllllll}3.43 & 18.46 & 0.078 & 0.614 & 0.618 & 0.493 & 0.112 & 0.337\end{array}$ $\begin{array}{lllllllll}3.63 & 18.08 & 0.078 & 0.619 & 0.642 & 0.504 & 0.084 & 0.318\end{array}$ $\begin{array}{lllllllll}3.39 & 18.23 & 0.089 & 0.660 & 0.710 & 0.522 & 0.104 & 0.386\end{array}$ $\begin{array}{llllllll}3.63 & 18.42 & 0.091 & 0.626 & 0.722 & 0.515 & 0.122 & 0.365\end{array}$ $\begin{array}{llllllll}3.40 & 18.50 & 0.091 & 0.654 & 0.683 & 0.515 & 0.102 & 0.366\end{array}$ $\begin{array}{lllllllll}3.52 & 18.49 & 0.089 & 0.637 & 0.715 & 0.537 & 0.098 & 0.357\end{array}$ $\begin{array}{llllllll}3.55 & 18.46 & 0.091 & 0.657 & 0.691 & 0.524 & 0.091 & 0.357\end{array}$ $\begin{array}{lllllllll}3.62 & 18.23 & 0.088 & 0.647 & 0.700 & 0.528 & 0.107 & 0.343\end{array}$ $\begin{array}{llllllll}3.60 & 18.12 & 0.082 & 0.633 & 0.648 & 0.479 & 0.134 & 0.424\end{array}$ $\begin{array}{lllllllll}3.57 & 18.48 & 0.079 & 0.617 & 0.641 & 0.484 & 0.096 & 0.384\end{array}$ $\begin{array}{lllllllll}3.52 & 18.65 & 0.079 & 0.625 & 0.644 & 0.489 & 0.125 & 0.422\end{array}$ $\begin{array}{llllllllll}3.71 & 18.24 & 0.100 & 0.592 & 0.712 & 0.400 & 0.106 & 0.365\end{array}$ $\begin{array}{llllllll}3.43 & 18.30 & 0.105 & 0.620 & 0.723 & 0.415 & 0.121 & 0.425\end{array}$ $\begin{array}{llllllll}3.50 & 18.49 & 0.099 & 0.599 & 0.734 & 0.386 & 0.117 & 0.382\end{array}$ $\begin{array}{lllllllll}3.50 & 18.42 & 0.082 & 0.638 & 0.664 & 0.510 & 0.095 & 0.338\end{array}$ $\begin{array}{llllllll}3.63 & 18.85 & 0.083 & 0.626 & 0.682 & 0.510 & 0.100 & 0.329\end{array}$ $\begin{array}{lllllllll}3.52 & 18.31 & 0.084 & 0.634 & 0.649 & 0.503 & 0.101 & 0.346\end{array}$ $\begin{array}{lllllllll}3.51 & 18.47 & 0.140 & 0.467 & 0.562 & 0.202 & 0.163 & 0.249\end{array}$
$52.33 \quad 3.54$ $52.54 \quad 3.52$ $\begin{array}{lllllll}41.27 & 0.159 & 1.172 & 0.320 & 0.170 & 0.269 & 0.156\end{array}$ $\begin{array}{lllllll}41.50 & 0.166 & 1.170 & 0.353 & 0.165 & 0.276 & 0.156\end{array}$ $\begin{array}{lllllll}42.30 & 0.176 & 0.546 & 0.733 & 0.375 & 0.146 & 0.416 \\ 42.33 & 0.175 & 0.552 & 0.726 & 0.381 & 0.133 & 0.392\end{array}$ $\begin{array}{llllllll}42.05 & 0.175 & 0.555 & 0.722 & 0.382 & 0.152 & 0.428\end{array}$ $\begin{array}{lllll}42.05 & 0.148 & 0.641 & 0.917\end{array}$ $\begin{array}{llllllll}42.05 & 0.148 & 0.641 & 0.917 & 0.669 & 0.127 & 0.442\end{array}$ $\begin{array}{llllllll}42.28 & 0.149 & 0.630 & 0.875 & 0.675 & 0.115 & 0.447\end{array}$ $\begin{array}{llllllll}42.69 & 0.147 & 0.637 & 0.919 & 0.658 & 0.117 & 0.432\end{array}$ $\begin{array}{llllllll}42.71 & 0.145 & 0.624 & 0.890 & 0.657 & 0.123 & 0.485\end{array}$ $\begin{array}{lllllll}41.36 & 0.137 & 0.634 & 0.855 & 0.645 & 0.145 & 0.493\end{array}$ $\begin{array}{lllllll}42.81 & 0.143 & 0.626 & 0.862 & 0.642 & 0.151 & 0.477\end{array}$ $\begin{array}{llllllll}42.26 & 0.139 & 0.616 & 0.870 & 0.658 & 0.121 & 0.417\end{array}$ $\begin{array}{llllllll}41.73 & 0.141 & 0.627 & 0.852 & 0.653 & 0.121 & 0.452\end{array}$ $\begin{array}{llllllll}41.89 & 0.138 & 0.618 & 0.863 & 0.643 & 0.105 & 0.416\end{array}$ $\begin{array}{llllllll}42.05 & 0.144 & 0.617 & 0.804 & 0.640 & 0.132 & 0.411\end{array}$ $\begin{array}{lllllllll}42.05 & 0.144 & 0.617 & 0.804 & 0.643 & 0.105 & 0.416 \\ 41.78 & 0.135 & 0.611 & 0.838 & 0.637 & 0.132 & 0.411\end{array}$ $\begin{array}{llllllll}41.78 & 0.135 & 0.611 & 0.838 & 0.637 & 0.126 & 0.393\end{array}$ $\begin{array}{llllllll}42.28 & 0.137 & 0.626 & 0.826 & 0.645 & 0.104 & 0.408\end{array}$ $\begin{array}{llllllll}42.23 & 0.142 & 0.628 & 0.860 & 0.644 & 0.112 & 0.442\end{array}$ $\begin{array}{lllllll}42.39 & 0.145 & 0.637 & 0.849 & 0.635 & 0.118 & 0.434\end{array}$ $\begin{array}{llllllll}42.33 & 0.144 & 0.611 & 0.896 & 0.645 & 0.110 & 0.460\end{array}$ $\begin{array}{llllllll}42.33 & 0.151 & 0.544 & 0.805 & 0.511 & 0.120 & 0.454\end{array}$ $\begin{array}{llllllll}41.68 & 0.144 & 0.538 & 0.808 & 0.512 & 0.097 & 0.427\end{array}$ $\begin{array}{llllllll}43.29 & 0.150 & 0.548 & 0.800 & 0.520 & 0.105 & 0.429\end{array}$ $\begin{array}{llllllllll}43.29 & 0.150 & 0.548 & 0.800 & 0.520 & 0.105 & 0.429\end{array}$ $41.30-0.198 \quad 0.426$ $\begin{array}{llllllll}41.80 & 0.198 & 0.438 & 0.816 & 0.320 & 0.167 & 0.433\end{array}$ $\begin{array}{llllllll}41.84 & 0.194 & 0.425 & 0.809 & 0.311 & 0.167 & 0.412\end{array}$ $\begin{array}{llllllll}42.19 & 0.141 & 0.625 & 0.864 & 0.653 & 0.128 & 0.459\end{array}$ $\begin{array}{lllllll}42.35 & 0.145 & 0.651 & 0.958 & 0.646 & 0.139 & 0.518\end{array}$ $\begin{array}{llllllll}41.48 & 0.145 & 0.654 & 0.870 & 0.657 & 0.115 & 0.441\end{array}$ $\begin{array}{llllllll}42.14 & 0.129 & 0.608 & 0.831 & 0.633 & 0.122 & 0.412\end{array}$ $\begin{array}{llllllll}42.30 & 0.129 & 0.614 & 0.795 & 0.636 & 0.132 & 0.427\end{array}$ $\begin{array}{llllllll}41.43 & 0.129 & 0.619 & 0.827 & 0.650 & 0.098 & 0.404\end{array}$ $\begin{array}{lllllllll}41.78 & 0.147 & 0.660 & 0.914 & 0.673 & 0.122 & 0.490\end{array}$ $\begin{array}{lllllllll}41.78 & 0.147 & 0.660 & 0.914 & 0.673 & 0.122 & 0.490\end{array}$ $\begin{array}{llllllll}42.21 & 0.151 & 0.626 & 0.929 & 0.665 & 0.143 & 0.464\end{array}$ $\begin{array}{llllllll}42.39 & 0.151 & 0.654 & 0.878 & 0.665 & 0.120 & 0.465\end{array}$ $\begin{array}{lllllll}42.37 & 0.148 & 0.637 & 0.920 & 0.693 & 0.115 & 0.453\end{array}$ $\begin{array}{llllllll}42.30 & 0.151 & 0.657 & 0.889 & 0.677 & 0.106 & 0.453\end{array}$ $\begin{array}{llllllll}41.78 & 0.145 & 0.647 & 0.900 & 0.682 & 0.125 & 0.436\end{array}$ $\begin{array}{llllllll}41.52 & 0.136 & 0.633 & 0.834 & 0.618 & 0.157 & 0.539\end{array}$ $\begin{array}{llllllll}42.35 & 0.131 & 0.617 & 0.824 & 0.625 & 0.112 & 0.487\end{array}$ $\begin{array}{llllllll}42.74 & 0.132 & 0.625 & 0.829 & 0.631 & 0.146 & 0.536\end{array}$ $\begin{array}{lllll}41.80 & 0.132 & 0.625 & 0.829\end{array}$ $\begin{array}{llllllll}41.80 & 0.166 & 0.592 & 0.916 & 0.516 & 0.124 & 0.464\end{array}$ $\begin{array}{llllllll}41.94 & 0.173 & 0.620 & 0.931 & 0.535 & 0.141 & 0.540\end{array}$ $\begin{array}{llllllll}42.37 & 0.165 & 0.599 & 0.944 & 0.498 & 0.137 & 0.485\end{array}$ $\begin{array}{lllllll}42.21 & 0.136 & 0.638 & 0.854 & 0.658 & 0.112 & 0.429\end{array}$ $\begin{array}{lllllll}43.20 & 0.138 & 0.626 & 0.878 & 0.659 & 0.117 & 0.418\end{array}$ $\begin{array}{lllllll}41.96 & 0.139 & 0.634 & 0.835 & 0.650 & 0.119 & 0.439\end{array}$ $\begin{array}{lllllll}42.33 & 0.233 & 0.467 & 0.723 & 0.261 & 0.191 & 0.316\end{array}$
99.4

99.8

100.5
100.4
99.9

99.9

100.2

100.5

101.0

100.3

99.9

100.8

100.6

100.1

99.7

100.3

100.0

101.1

101.

100.4

99.7

101.4

100.6

99.9

100.3

100.6

101.0

99.5

100.4

100.5

99.8

100.8

100.6

101.2

101.5

100.0

100.9

101.3

100.8

100.9

100.3

101.6

100.6

99.6 
$798 \quad 36.80$ $799 \quad 36.86$ 80036.98 $801 \quad 36.95$ $802 \quad 36.60$ $803 \quad 36.70$ $805 \quad 36.90$ 80636.82 80736.68 $808 \quad 36.76$ $809 \quad 37.02$ $810 \quad 37.12$ $811 \quad 37.18$ $812 \quad 37.14$ $813 \quad 37.08$

Vasagå $816 \quad 37.34$

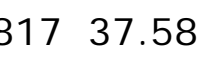
$818 \quad 37.35$ $\begin{array}{lll}819 & 37.24\end{array}$ 82037.01 82136.97 $822 \quad 37.29$ $823 \quad 37.32$ 82437.50 $825 \quad 37.20$ $826 \quad 37.24$ 82737.04 $828 \quad 36.74$ 82936.99 83036.95 $\begin{array}{ll}831 & 36.93 \\ 832 & 37.08\end{array}$ $\begin{array}{lll}832 & 37.08\end{array}$ $833 \quad 37.02$ 83437.05 83537.30 83637.24 $\begin{array}{lll}837 & 36.89\end{array}$ $838 \quad 37.28$ 83937.38 84037.31 84137.45 $842 \quad 37.15$ $843 \quad 37.70$ $\begin{array}{lll}844 & 38.47\end{array}$ $845 \quad 37.67$ $846 \quad 37.32$ $\begin{array}{llllllll}3.68 & 18.42 & 0.142 & 0.462 & 0.531 & 0.199 & 0.152 & 0.268\end{array}$ $\begin{array}{lllllllll}3.48 & 18.18 & 0.138 & 0.464 & 0.549 & 0.207 & 0.143 & 0.256\end{array}$ $\begin{array}{lllllllll}3.55 & 17.89 & 0.086 & 0.615 & 0.658 & 0.511 & 0.098 & 0.339\end{array}$ $\begin{array}{lllllllll}3.60 & 18.20 & 0.083 & 0.624 & 0.693 & 0.494 & 0.090 & 0.325\end{array}$ $\begin{array}{lllllllll}3.60 & 18.41 & 0.088 & 0.618 & 0.650 & 0.508 & 0.114 & 0.353\end{array}$ $\begin{array}{lllllllll}3.48 & 17.94 & 0.086 & 0.604 & 0.654 & 0.508 & 0.108 & 0.346\end{array}$ $\begin{array}{lllllllll}3.57 & 18.52 & 0.087 & 0.629 & 0.676 & 0.494 & 0.104 & 0.354\end{array}$ $\begin{array}{lllllllll}3.61 & 18.53 & 0.089 & 0.610 & 0.697 & 0.500 & 0.112 & 0.359\end{array}$ $\begin{array}{llllllll}3.36 & 18.58 & 0.090 & 0.618 & 0.673 & 0.509 & 0.104 & 0.376\end{array}$ $\begin{array}{lllllllll}3.52 & 18.05 & 0.082 & 0.629 & 0.684 & 0.513 & 0.107 & 0.386\end{array}$ $\begin{array}{llllllll}3.55 & 18.26 & 0.084 & 0.622 & 0.669 & 0.508 & 0.117 & 0.384\end{array}$ $\begin{array}{lllllllll}3.48 & 18.08 & 0.082 & 0.616 & 0.664 & 0.491 & 0.111 & 0.365\end{array}$ $\begin{array}{lllllllll}3.55 & 18.56 & 0.085 & 0.615 & 0.663 & 0.501 & 0.115 & 0.375\end{array}$ $\begin{array}{lllllllll}3.56 & 18.49 & 0.083 & 0.601 & 0.649 & 0.499 & 0.107 & 0.306\end{array}$ $\begin{array}{lllllllll}3.58 & 18.32 & 0.091 & 0.644 & 0.669 & 0.494 & 0.105 & 0.330\end{array}$ $\begin{array}{llllllll}3.45 & 18.24 & 0.089 & 0.638 & 0.698 & 0.502 & 0.089 & 0.324 \\ 3.53 & 18.39 & 0.087 & 0.633 & 0.678 & 0.512 & 0.101 & 0.337\end{array}$ $\begin{array}{ll}3.53 & 18.39 \\ 3.34 & 18.42\end{array}$

$\begin{array}{lllllllll}3.34 & 18.42 & 0.091 & 0.551 & 0.646 & 0.387 & 0.106 & 0.353\end{array}$ $\begin{array}{lllllllll}3.41 & 18.74 & 0.093 & 0.556 & 0.623 & 0.386 & 0.084 & 0.374\end{array}$ $\begin{array}{lllllllll}3.60 & 18.33 & 0.090 & 0.553 & 0.635 & 0.383 & 0.113 & 0.357\end{array}$ $\begin{array}{lllllllll}3.53 & 18.36 & 0.088 & 0.645 & 0.671 & 0.494 & 0.117 & 0.350\end{array}$ $\begin{array}{lllllllll}3.38 & 18.67 & 0.088 & 0.644 & 0.714 & 0.509 & 0.095 & 0.333\end{array}$ $\begin{array}{llllllll}3.73 & 18.30 & 0.090 & 0.632 & 0.678 & 0.521 & 0.122 & 0.349\end{array}$ $\begin{array}{lllllllll}3.53 & 18.59 & 0.086 & 0.629 & 0.682 & 0.509 & 0.099 & 0.325\end{array}$ $\begin{array}{lllllllll}3.59 & 18.16 & 0.086 & 0.649 & 0.667 & 0.500 & 0.108 & 0.342\end{array}$ $\begin{array}{lllllllll}3.64 & 18.63 & 0.087 & 0.641 & 0.687 & 0.515 & 0.108 & 0.338\end{array}$ $\begin{array}{lllllllll}3.54 & 18.28 & 0.088 & 0.601 & 0.677 & 0.503 & 0.103 & 0.334\end{array}$ $\begin{array}{llllllll}3.54 & 18.41 & 0.082 & 0.598 & 0.684 & 0.508 & 0.103 & 0.337\end{array}$ $\begin{array}{lllllllll}3.58 & 18.54 & 0.087 & 0.588 & 0.685 & 0.497 & 0.111 & 0.338\end{array}$ $\begin{array}{lllllllll}3.58 & 18.40 & 0.085 & 0.580 & 0.689 & 0.512 & 0.111 & 0.348\end{array}$ $\begin{array}{lllllllll}3.49 & 18.59 & 0.086 & 0.580 & 0.673 & 0.506 & 0.105 & 0.343\end{array}$ $\begin{array}{llllllll}3.51 & 18.23 & 0.092 & 0.585 & 0.702 & 0.504 & 0.108 & 0.341\end{array}$ $\begin{array}{llllllll}3.48 & 18.38 & 0.084 & 0.608 & 0.655 & 0.494 & 0.122 & 0.385\end{array}$ $\begin{array}{llllllll}3.64 & 18.33 & 0.084 & 0.646 & 0.666 & 0.500 & 0.105 & 0.357\end{array}$ $\begin{array}{llllllll}3.38 & 18.72 & 0.081 & 0.618 & 0.657 & 0.494 & 0.114 & 0.355\end{array}$ $\begin{array}{llllllll}3.32 & 18.15 & 0.099 & 0.515 & 0.618 & 0.298 & 0.103 & 0.295\end{array}$ $\begin{array}{llllllll}3.64 & 18.41 & 0.104 & 0.488 & 0.619 & 0.294 & 0.117 & 0.293\end{array}$ $\begin{array}{lllllllll}3.38 & 18.21 & 0.099 & 0.504 & 0.611 & 0.301 & 0.108 & 0.292\end{array}$ $\begin{array}{llllllll}3.60 & 18.13 & 0.092 & 0.535 & 0.607 & 0.395 & 0.087 & 0.322\end{array}$ $\begin{array}{llllllll}3.56 & 18.28 & 0.089 & 0.552 & 0.658 & 0.394 & 0.127 & 0.348\end{array}$ $\begin{array}{lllllllll}3.53 & 18.33 & 0.089 & 0.530 & 0.614 & 0.391 & 0.120 & 0.357\end{array}$ $\begin{array}{lllllllll}3.72 & 18.65 & 0.107 & 0.528 & 0.613 & 0.287 & 0.115 & 0.311\end{array}$ $\begin{array}{llllllll}3.58 & 18.69 & 0.107 & 0.530 & 0.593 & 0.288 & 0.119 & 0.318\end{array}$ $\begin{array}{llllllll}3.44 & 18.42 & 0.105 & 0.543 & 0.600 & 0.286 & 0.129 & 0.313\end{array}$ $\begin{array}{lllllllll}3.55 & 18.48 & 0.051 & 0.678 & 0.217 & 0.220 & 0.227 & 0.181\end{array}$ $\begin{array}{llllllll}3.43 & 18.91 & 0.003 & 0.307 & 0.000 & 0.000 & 2.928 & 1.514\end{array}$ $\begin{array}{lllllllll}3.60 & 17.99 & 0.049 & 0.715 & 0.195 & 0.195 & 0.201 & 0.155\end{array}$ $\begin{array}{lllllllll}3.41 & 18.39 & 0.087 & 0.600 & 0.701 & 0.506 & 0.103 & 0.344\end{array}$
$51.52 \quad 3.68$

$1.60 \quad 3.48$

$51.77 \quad 3.55$

51.73

$51.38 \quad 3.48$

$51.53 \quad 3.57$

$51.66 \quad 3.61$

$51.55 \quad 3.36$

$\begin{array}{ll}51.35 & 3.52 \\ 5 & \end{array}$

$1.46 \quad 3.55$

$51.83 \quad 3.48$

$\begin{array}{ll}51.97 & 3.55 \\ 52.05 & 3.56 \\ 52.00 & 3.58\end{array}$

$52.00 \quad 3.58$

$52.00 \quad 3.58$

$51.81 \quad 3.53$

$52.28 \quad 3.34$

$52.61 \quad 3.41$

$52.29 \quad 3.60$

$\begin{array}{ll}52.14 & 3.53 \\ 51.81 & 3.38\end{array}$

$\begin{array}{ll}51.76 & 3.73\end{array}$

$\begin{array}{ll}51.76 & 3.73 \\ 52.21 & 3.53\end{array}$

$\begin{array}{ll}52.25 & 3.59\end{array}$

$52.50 \quad 3.64$

$\begin{array}{ll}52.08 & 3.54\end{array}$

$52.14 \quad 3.54$

$51.86 \quad 3.58$

$51.44 \quad 3.58$

$51.79 \quad 3.49$

$51.73 \quad 3.51$

$51.70 \quad 3.48$

$51.91 \quad 3.64$

$51.83 \quad 3.38$

$\begin{array}{ll}51.87 & 3.32\end{array}$

$52.22 \quad 3.64$

$\begin{array}{ll}52.14 & 3.38 \\ 51.65 & 3.60 \\ 52.19 & 3.56\end{array}$

$52.19 \quad 3.56$

$52.33 \quad 3.53$

$52.23 \quad 3.72$

$52.43 \quad 3.58$

$\begin{array}{ll}52.01 & 3.44\end{array}$

$52.78 \quad 3.55$

$53.86 \quad 3.43$

$\begin{array}{ll}52.74 & 3.60 \\ 52.25 & 3.41\end{array}$ $\begin{array}{llllllll}2.21 & 0.236 & 0.462 & 0.683 & 0.257 & 0.178 & 0.341\end{array}$ $\begin{array}{lllllll}41.66 & 0.229 & 0.464 & 0.707 & 0.267 & 0.167 & 0.325\end{array}$ $\begin{array}{lllllll}41.00 & 0.143 & 0.615 & 0.847 & 0.660 & 0.115 & 0.430\end{array}$ $\begin{array}{llllllll}41.71 & 0.138 & 0.624 & 0.892 & 0.638 & 0.105 & 0.413\end{array}$ $\begin{array}{llllllll}42.19 & 0.145 & 0.618 & 0.836 & 0.656 & 0.134 & 0.448\end{array}$ $\begin{array}{llllllll}41.11 & 0.142 & 0.604 & 0.841 & 0.656 & 0.127 & 0.439\end{array}$ $\begin{array}{llllllll}42.44 & 0.143 & 0.629 & 0.869 & 0.638 & 0.127 & 0.439\end{array}$ \begin{tabular}{lllllllll}
41.141 & 0.142 & 0.604 & 0.841 & 0.656 & 0.127 & 0.439 \\
\hline 4.46 & 0.147 & 0.629 & 0.869 & 0.638 & 0.121 & 0.449
\end{tabular} $\begin{array}{llllllll}4.46 & 0.147 & 0.610 & 0.896 & 0.645 & 0.132 & 0.456\end{array}$ $\begin{array}{lllllllll}42.46 & 0.147 & 0.610 & 0.896 & 0.645 & 0.132 & 0.456\end{array}$ $\begin{array}{llllllll}1.36 & 0.136 & 0.629 & 0.880 & 0.663 & 0.126 & 0.490\end{array}$ $\begin{array}{llllllll}41.84 & 0.138 & 0.622 & 0.861 & 0.656 & 0.136 & 0.488\end{array}$ $\begin{array}{llllllll}41.43 & 0.136 & 0.616 & 0.854 & 0.634 & 0.130 & 0.464\end{array}$ $\begin{array}{llllllll}42.53 & 0.140 & 0.615 & 0.853 & 0.647 & 0.135 & 0.476\end{array}$ $\begin{array}{llllllll}42.37 & 0.138 & 0.601 & 0.835 & 0.644 & 0.125 & 0.389\end{array}$ $\begin{array}{llllllll}41.98 & 0.150 & 0.644 & 0.861 & 0.638 & 0.123 & 0.419\end{array}$ $\begin{array}{llllllll}41.80 & 0.148 & 0.638 & 0.898 & 0.648 & 0.104 & 0.412\end{array}$ $\begin{array}{lllllll}42.14 & 0.145 & 0.633 & 0.872 & 0.661 & 0.118 & 0.428\end{array}$

$\begin{array}{lllllll}42.21 & 0.151 & 0.551 & 0.831 & 0.500 & 0.124 & 0.449\end{array}$ $\begin{array}{llllllll}42.94 & 0.154 & 0.556 & 0.802 & 0.499 & 0.098 & 0.475\end{array}$ $\begin{array}{llllllll}42.00 & 0.149 & 0.553 & 0.818 & 0.495 & 0.132 & 0.453\end{array}$ $\begin{array}{llllllll}42.07 & 0.146 & 0.645 & 0.863 & 0.638 & 0.136 & 0.445\end{array}$ $\begin{array}{llllllll}42.78 & 0.146 & 0.644 & 0.919 & 0.657 & 0.111 & 0.423\end{array}$ $\begin{array}{llllllll}42.78 & 0.146 & 0.644 & 0.919 & 0.657 & 0.111 & 0.423\end{array}$ $\begin{array}{llllllll}42.60 & 0.142 & 0.629 & 0.878 & 0.657 & 0.116 & 0.412\end{array}$ $\begin{array}{llllllll}42.60 & 0.142 & 0.629 & 0.878 & 0.657 & 0.116 & 0.412\end{array}$ $\begin{array}{lllllll}41.61 & 0.143 & 0.649 & 0.858 & 0.645 & 0.127 & 0.434\end{array}$ $\begin{array}{lllllllll}41.61 & 0.143 & 0.649 & 0.858 & 0.645 & 0.127 & 0.434\end{array}$ $\begin{array}{llllllll}41.89 & 0.145 & 0.601 & 0.872 & 0.650 & 0.121 & 0.424\end{array}$ $\begin{array}{llllllll}42.19 & 0.137 & 0.598 & 0.880 & 0.655 & 0.120 & 0.428\end{array}$ $\begin{array}{llllllll}42.49 & 0.145 & 0.588 & 0.881 & 0.642 & 0.130 & 0.430\end{array}$ $\begin{array}{llllllll}42.16 & 0.140 & 0.580 & 0.887 & 0.661 & 0.130 & 0.441\end{array}$ $\begin{array}{llllllll}42.60 & 0.143 & 0.580 & 0.866 & 0.653 & 0.123 & 0.435\end{array}$ $\begin{array}{lllllll}42.60 & 0.143 & 0.580 & 0.866 & 0.653 & 0.123 & 0.435 \\ 41.78 & 0.152 & 0.585 & 0.903 & 0.651 & 0.126 & 0.434\end{array}$ $\begin{array}{lllllllllll}42.12 & 0.140 & 0.608 & 0.843 & 0.638 & 0.143 & 0.489\end{array}$ $\begin{array}{lll}42.12-0.140 & 0.608 & 0.843\end{array}$ $\begin{array}{llllllll}42.00 & 0.139 & 0.646 & 0.857 & 0.645 & 0.123 & 0.453\end{array}$ $\begin{array}{llllllll}42.90 & 0.135 & 0.618 & 0.845 & 0.638 & 0.134 & 0.451\end{array}$ $\begin{array}{llllllll}41.59 & 0.164 & 0.515 & 0.795 & 0.384 & 0.121 & 0.374\end{array}$ $\begin{array}{lllllll}42.19 & 0.172 & 0.488 & 0.796 & 0.380 & 0.137 & 0.373\end{array}$ $\begin{array}{llllllll}41.73 & 0.163 & 0.504 & 0.786 & 0.389 & 0.126 & 0.371\end{array}$ $\begin{array}{llllllll}41.55 & 0.153 & 0.535 & 0.781 & 0.510 & 0.102 & 0.409\end{array}$ $\begin{array}{llllllll}41.89 & 0.148 & 0.552 & 0.846 & 0.508 & 0.149 & 0.441\end{array}$ $\begin{array}{llllllll}42.00 & 0.148 & 0.530 & 0.790 & 0.505 & 0.141 & 0.453\end{array}$ $\begin{array}{llllllll}42.74 & 0.177 & 0.528 & 0.789 & 0.370 & 0.135 & 0.395\end{array}$ $\begin{array}{llllllll}42.83 & 0.178 & 0.530 & 0.763 & 0.372 & 0.139 & 0.404\end{array}$ $\begin{array}{llllllll}42.21 & 0.174 & 0.543 & 0.772 & 0.370 & 0.151 & 0.397\end{array}$ $\begin{array}{llllllll}4.35 & 0.085 & 0.678 & 0.279 & 0.283 & 0.266 & 0.230\end{array}$ $\begin{array}{llllllll}43.33 & 0.005 & 0.307 & 0.000 & 0.000 & 3.429 & 1.923\end{array}$ $\begin{array}{llllllll}41.23 & 0.081 & 0.715 & 0.250 & 0.252 & 0.235 & 0.197\end{array}$ $\begin{array}{llllllll}42.14 & 0.145 & 0.600 & 0.902 & 0.654 & 0.120 & 0.436\end{array}$
98.9

99.1

99.9

98.8

98.8

$(00.4$

100.6

100.4

99.8

99.6

100.9

100.7

100.4

100.3

100.4

101.5

100.5

100.6

100.9

100.3

101.7

100.3

100.7

100.7

100.0

100.7

99.9

100.2

100.4

100.9

99.1

100.4

100.3

100.4

101.2

100.1

100.5

106.3 
84737.06 $848 \quad 37.20$ 84936.93 $850 \quad 36.78$ 85136.80 $852 \quad 37.19$ $\begin{array}{lll}852 & 37.19\end{array}$ 36.76 $\begin{array}{lll}854 & 37.11 \\ 855 & 37.43\end{array}$ 85537.43 85637.23 $857 \quad 37.33$ $858 \quad 37.30$ $\begin{array}{lll}859 & 37.22\end{array}$ 86037.09 86136.80 $\begin{array}{lll}863 & 36.59\end{array}$ $\begin{array}{lll}864 & 36.77\end{array}$ $\begin{array}{ll}865 & 36.79\end{array}$ $\begin{array}{ll}866 & 37.09\end{array}$ $\begin{array}{lll}867 & 37.07\end{array}$ $868 \quad 36.89$ 86937.06 87036.75 87036.75 $872 \quad 36.94$ $873 \quad 36.93$ $874 \quad 36.95$ $\begin{array}{lll}875 & 36.77\end{array}$ Vasagård $\begin{array}{ll}876 & 37.10 \\ 877 & 37.12\end{array}$ $\begin{array}{lll}877 & 37.12 \\ 878 & 37.89\end{array}$ $878 \quad 37.89$ 87936.93 88037.06 $\begin{array}{lll}881 & 36.99\end{array}$ 88237.09 88337.06 $\begin{array}{lll}884 & 37.27\end{array}$ $\begin{array}{ll}885 & 37.51\end{array}$ \begin{tabular}{l}
$886 \quad 37.67$ \\
\hline
\end{tabular} $887 \quad 37.35$ 88837.23 88936.84 89036.99 $891 \quad 36.87$ $892 \quad 36.87$ $893 \quad 37.11$ $894 \quad 37.27$ $\begin{array}{llllllll}3.55 & 18.17 & 0.086 & 0.590 & 0.681 & 0.521 & 0.106 & 0.354\end{array}$ $\begin{array}{llllllll}3.64 & 18.23 & 0.086 & 0.606 & 0.682 & 0.511 & 0.106 & 0.335\end{array}$ $\begin{array}{lllllllll}3.45 & 18.34 & 0.091 & 0.639 & 0.713 & 0.528 & 0.096 & 0.371\end{array}$ $\begin{array}{lllllllll}3.46 & 18.16 & 0.089 & 0.623 & 0.707 & 0.537 & 0.106 & 0.361\end{array}$ $\begin{array}{lllllllll}3.57 & 18.35 & 0.085 & 0.623 & 0.701 & 0.537 & 0.096 & 0.355\end{array}$ $\begin{array}{lllllllll}3.59 & 18.56 & 0.082 & 0.592 & 0.665 & 0.493 & 0.091 & 0.305\end{array}$ $\begin{array}{llllllll}3.41 & 18.33 & 0.082 & 0.595 & 0.636 & 0.488 & 0.090 & 0.309\end{array}$ $\begin{array}{lllllllll}3.53 & 18.52 & 0.080 & 0.601 & 0.648 & 0.495 & 0.093 & 0.321\end{array}$ $\begin{array}{llllllll}3.52 & 18.42 & 0.084 & 0.511 & 0.596 & 0.373 & 0.099 & 0.321\end{array}$ $\begin{array}{llllllll}3.67 & 18.39 & 0.087 & 0.515 & 0.626 & 0.384 & 0.121 & 0.311\end{array}$ $\begin{array}{lllllllll}3.57 & 18.40 & 0.087 & 0.530 & 0.593 & 0.375 & 0.132 & 0.319\end{array}$ $\begin{array}{llllllll}3.57 & 18.57 & 0.091 & 0.532 & 0.628 & 0.372 & 0.110 & 0.322\end{array}$ $\begin{array}{lllllllll}3.66 & 18.37 & 0.087 & 0.539 & 0.608 & 0.373 & 0.082 & 0.324\end{array}$ $\begin{array}{lllllllll}3.69 & 18.70 & 0.094 & 0.525 & 0.647 & 0.378 & 0.098 & 0.322\end{array}$ $\begin{array}{lllllllll}3.65 & 18.39 & 0.076 & 0.606 & 0.644 & 0.500 & 0.104 & 0.323\end{array}$ $\begin{array}{lllllllll}3.58 & 18.49 & 0.079 & 0.600 & 0.646 & 0.491 & 0.092 & 0.318\end{array}$ $\begin{array}{llllllll}3.48 & 18.49 & 0.081 & 0.604 & 0.644 & 0.492 & 0.088 & 0.321\end{array}$ $\begin{array}{lllllllll}3.60 & 18.47 & 0.082 & 0.610 & 0.696 & 0.495 & 0.092 & 0.333\end{array}$ $\begin{array}{lllllllll}3.46 & 18.44 & 0.086 & 0.611 & 0.635 & 0.498 & 0.117 & 0.327\end{array}$ $\begin{array}{lllllllll}3.63 & 18.31 & 0.083 & 0.594 & 0.681 & 0.491 & 0.087 & 0.327\end{array}$ $\begin{array}{lllllllll}3.51 & 18.35 & 0.087 & 0.545 & 0.636 & 0.390 & 0.122 & 0.360\end{array}$ $\begin{array}{lllllllll}3.52 & 18.40 & 0.087 & 0.545 & 0.632 & 0.389 & 0.140 & 0.376\end{array}$ $\begin{array}{lllllllll}3.61 & 18.24 & 0.008 & 0.102 & 0.052 & 0.026 & 0.114 & 0.178\end{array}$ $\begin{array}{lllllllll}3.58 & 18.18 & 0.086 & 0.604 & 0.691 & 0.531 & 0.095 & 0.312\end{array}$ $\begin{array}{lllllllll}3.67 & 18.22 & 0.086 & 0.584 & 0.663 & 0.528 & 0.114 & 0.321\end{array}$ $\begin{array}{lllllllll}3.52 & 18.16 & 0.087 & 0.589 & 0.707 & 0.535 & 0.097 & 0.318\end{array}$ $\begin{array}{lllllllll}3.76 & 18.39 & 0.087 & 0.637 & 0.679 & 0.520 & 0.106 & 0.356\end{array}$ $\begin{array}{lllllllll}3.57 & 18.39 & 0.085 & 0.639 & 0.674 & 0.517 & 0.100 & 0.343\end{array}$ $\begin{array}{llllllll}3.53 & 18.33 & 0.088 & 0.645 & 0.671 & 0.513 & 0.125 & 0.379\end{array}$ nekulle \#6

$\begin{array}{llllllll}3.52 & 18.52 & 0.149 & 0.468 & 0.587 & 0.204 & 0.144 & 0.245\end{array}$ $\begin{array}{lllllllll}3.59 & 18.73 & 0.153 & 0.459 & 0.568 & 0.198 & 0.157 & 0.243\end{array}$ $\begin{array}{lllllllll}3.44 & 18.49 & 0.151 & 0.466 & 0.574 & 0.200 & 0.154 & 0.237\end{array}$ $\begin{array}{lllllllll}3.40 & 18.11 & 0.094 & 0.542 & 0.625 & 0.405 & 0.126 & 0.348\end{array}$ $\begin{array}{llllllll}3.40 & 18.39 & 0.091 & 0.545 & 0.644 & 0.399 & 0.101 & 0.354\end{array}$ $\begin{array}{llllllll}3.60 & 18.61 & 0.090 & 0.550 & 0.607 & 0.398 & 0.097 & 0.357\end{array}$ $\begin{array}{llllllll}3.60 & 18.34 & 0.094 & 0.554 & 0.647 & 0.396 & 0.129 & 0.391\end{array}$ $\begin{array}{lllllllll}3.60 & 18.27 & 0.092 & 0.541 & 0.631 & 0.398 & 0.148 & 0.419\end{array}$ $\begin{array}{lllllllll}3.43 & 18.43 & 0.097 & 0.612 & 0.677 & 0.411 & 0.127 & 0.397\end{array}$ $\begin{array}{lllllllll}3.64 & 18.93 & 0.081 & 0.602 & 0.627 & 0.498 & 0.096 & 0.300\end{array}$ $\begin{array}{lllllllll}3.61 & 18.71 & 0.079 & 0.625 & 0.640 & 0.484 & 0.098 & 0.307\end{array}$ $\begin{array}{lllllllll}3.63 & 18.68 & 0.080 & 0.613 & 0.585 & 0.481 & 0.086 & 0.318\end{array}$ $\begin{array}{lllllllll}3.54 & 18.37 & 0.086 & 0.640 & 0.671 & 0.513 & 0.104 & 0.345\end{array}$ $\begin{array}{llllllll}3.48 & 18.38 & 0.088 & 0.639 & 0.656 & 0.512 & 0.112 & 0.326\end{array}$ $\begin{array}{lllllllll}3.47 & 18.53 & 0.088 & 0.616 & 0.689 & 0.502 & 0.102 & 0.322\end{array}$ $\begin{array}{lllllllll}3.53 & 18.38 & 0.089 & 0.622 & 0.656 & 0.500 & 0.114 & 0.362\end{array}$ $\begin{array}{lllllllll}3.41 & 18.53 & 0.083 & 0.627 & 0.670 & 0.490 & 0.091 & 0.340\end{array}$ $\begin{array}{lllllllll}3.53 & 18.25 & 0.088 & 0.603 & 0.655 & 0.484 & 0.099 & 0.351\end{array}$ $\begin{array}{llllllll}3.49 & 18.73 & 0.085 & 0.634 & 0.682 & 0.492 & 0.121 & 0.333\end{array}$ $\begin{array}{llllllll}41.64 & 0.142 & 0.590 & 0.876 & 0.673 & 0.124 & 0.450\end{array}$ $\begin{array}{lllllll}41.78 & 0.143 & 0.606 & 0.877 & 0.660 & 0.124 & 0.425\end{array}$ $\begin{array}{llllllll}42.03 & 0.151 & 0.639 & 0.917 & 0.682 & 0.112 & 0.471\end{array}$ $\begin{array}{lllllll}41.61 & 0.147 & 0.623 & 0.909 & 0.693 & 0.124 & 0.458\end{array}$ $\begin{array}{llllllll}42.05 & 0.141 & 0.623 & 0.901 & 0.694 & 0.113 & 0.451\end{array}$ $\begin{array}{llllllll}42.53 & 0.136 & 0.592 & 0.855 & 0.637 & 0.106 & 0.387\end{array}$ $\begin{array}{lllllll}42.00 & 0.136 & 0.595 & 0.818 & 0.629 & 0.106 & 0.392\end{array}$ $\begin{array}{llllllll}42.44 & 0.133 & 0.601 & 0.834 & 0.639 & 0.109 & 0.408\end{array}$ $\begin{array}{llllllll}42.21 & 0.138 & 0.511 & 0.767 & 0.482 & 0.116 & 0.408\end{array}$ $\begin{array}{lllllll}42.14 & 0.144 & 0.515 & 0.805 & 0.496 & 0.142 & 0.395\end{array}$ $\begin{array}{lllllll}42.16 & 0.144 & 0.530 & 0.763 & 0.484 & 0.155 & 0.405\end{array}$ $\begin{array}{lllllll}42.55 & 0.151 & 0.532 & 0.808 & 0.480 & 0.129 & 0.409\end{array}$ $\begin{array}{llllllll}42.10 & 0.145 & 0.539 & 0.782 & 0.482 & 0.096 & 0.411\end{array}$ $\begin{array}{llllllll}42.85 & 0.155 & 0.525 & 0.832 & 0.488 & 0.114 & 0.408\end{array}$ $\begin{array}{lllllll}42.14 & 0.127 & 0.606 & 0.828 & 0.646 & 0.122 & 0.410\end{array}$ $\begin{array}{llllllll}42.37 & 0.131 & 0.600 & 0.832 & 0.634 & 0.107 & 0.404\end{array}$ $\begin{array}{lllll}42.37 & 0.131 & 0.600 & 0.832\end{array}$ $\begin{array}{llllllll}42.37 & 0.134 & 0.604 & 0.829 & 0.636 & 0.103 & 0.408\end{array}$ $\begin{array}{llll}0.639 & 0.108 & 0.423\end{array}$ $\begin{array}{lllllll}42.26 & 0.143 & 0.611 & 0.817 & 0.643 & 0.137 & 0.415\end{array}$ $\begin{array}{lllllll}41.96 & 0.138 & 0.594 & 0.876 & 0.634 & 0.102 & 0.415\end{array}$ $\begin{array}{lllllll}42.05 & 0.145 & 0.545 & 0.818 & 0.504 & 0.143 & 0.457\end{array}$ $\begin{array}{llllllll}42.16 & 0.145 & 0.545 & 0.814 & 0.503 & 0.164 & 0.477\end{array}$ $\begin{array}{llllllll}41.80 & 0.014 & 0.102 & 0.067 & 0.033 & 0.133 & 0.226\end{array}$ $\begin{array}{lllllll}41.66 & 0.143 & 0.604 & 0.889 & 0.685 & 0.111 & 0.396\end{array}$ $\begin{array}{llllllll}41.75 & 0.143 & 0.584 & 0.853 & 0.682 & 0.134 & 0.407\end{array}$ $\begin{array}{lll}41.75 & 0.143 & 0.58\end{array}$ $\begin{array}{llllllll}41.61 & 0.144 & 0.589 & 0.909 & 0.690 & 0.114 & 0.404\end{array}$ $\begin{array}{llllllll}42.14 & 0.144 & 0.637 & 0.874 & 0.671 & 0.125 & 0.452\end{array}$ $\begin{array}{lllllll}42.14 & 0.140 & 0.639 & 0.868 & 0.667 & 0.117 & 0.436 \\ 42.00 & 0.146 & 0.645 & 0.864 & 0.663 & 0.147 & 0.481\end{array}$

$\begin{array}{lllllll}42.44 & 0.247 & 0.468 & 0.755 & 0.263 & 0.168 & 0.311\end{array}$ $\begin{array}{llllllll}42.92 & 0.254 & 0.459 & 0.731 & 0.255 & 0.184 & 0.309\end{array}$ $\begin{array}{llllllll}42.37 & 0.250 & 0.466 & 0.739 & 0.259 & 0.180 & 0.301\end{array}$ $\begin{array}{llllllll}41.50 & 0.155 & 0.542 & 0.804 & 0.523 & 0.148 & 0.442\end{array}$ $\begin{array}{llllllll}42.14 & 0.150 & 0.545 & 0.828 & 0.515 & 0.118 & 0.450\end{array}$ $\begin{array}{llllllll}42.65 & 0.149 & 0.550 & 0.781 & 0.514 & 0.114 & 0.453\end{array}$ $\begin{array}{llllllll}42.03 & 0.156 & 0.554 & 0.832 & 0.512 & 0.151 & 0.496\end{array}$ $\begin{array}{lllllll}41.87 & 0.152 & 0.541 & 0.812 & 0.514 & 0.173 & 0.532\end{array}$ $\begin{array}{lllllll}42.23 & 0.162 & 0.612 & 0.871 & 0.531 & 0.148 & 0.504\end{array}$ $\begin{array}{lllllll}43.38 & 0.134 & 0.602 & 0.807 & 0.643 & 0.113 & 0.381\end{array}$ $\begin{array}{lllllll}42.88 & 0.130 & 0.625 & 0.823 & 0.625 & 0.115 & 0.390\end{array}$ $\begin{array}{llllllll}42.81 & 0.132 & 0.613 & 0.753 & 0.621 & 0.100 & 0.404\end{array}$ $\begin{array}{llllllll}42.81 & 0.132 & 0.613 & 0.753 & 0.621 & 0.1100 & 0.404\end{array}$ $\begin{array}{llllllll}42.10 & 0.143 & 0.640 & 0.863 & 0.662 & 0.121 & 0.438\end{array}$ $\begin{array}{llllllll}42.12 & 0.145 & 0.639 & 0.844 & 0.661 & 0.131 & 0.414\end{array}$ $\begin{array}{llllllll}42.46 & 0.145 & 0.616 & 0.887 & 0.648 & 0.120 & 0.408\end{array}$ $\begin{array}{lllllll}42.12 & 0.148 & 0.622 & 0.843 & 0.646 & 0.134 & 0.459\end{array}$ $\begin{array}{llllllll}42.46 & 0.137 & 0.627 & 0.862 & 0.633 & 0.107 & 0.432\end{array}$ $\begin{array}{lllllll}41.82 & 0.145 & 0.603 & 0.842 & 0.624 & 0.116 & 0.445\end{array}$ $\begin{array}{llllllll}42.92 & 0.140 & 0.634 & 0.877 & 0.636 & 0.142 & 0.423\end{array}$
99.9 100.3 100.2

100.9

100.6

100.6

100.4

100.5

100.9

100.3

101.0

99.8

00.2

100.0
100.3

100.1

100.0

97.9

99.5

99.9

99.7

100.5

100.3

100.0

100.1

100.7

.

100.0

100.6

100.3

100.1

100.7

102.2

101.9

100.0

100.5

100.1

100.3

100.1 
89537.39 $896 \quad 37.02$
897 $897 \quad 37.34$ $898 \quad 37.71$ 89937.43 $900 \quad 37.42$ $\begin{array}{lll}901 & 37.69\end{array}$ $902 \quad 37.16$ $903 \quad 36.99$ 90437.10 $905 \quad 37.31$ $\begin{array}{lll}906 & 37.27\end{array}$ $907 \quad 37.49$ $908 \quad 37.42$ $909 \quad 37.52$ $910 \quad 37.70$ $911 \quad 37.68$ $912 \quad 37.24$ $913 \quad 37.70$ $914 \quad 37.73$ 91537.66 $916 \quad 37.81$ $917 \quad 37.82$ $918 \quad 37.22$ $919 \quad 37.21$ $920 \quad 37.32$ $921 \quad 37.36$ 92237.46 $923 \quad 37.48$ $924 \quad 37.24$ $\begin{array}{ll}925 & 0.56\end{array}$ $926 \quad 37.30$ $927 \quad 37.21$ $\begin{array}{lll}927 & 37.21 \\ 928 & 37.22\end{array}$ $\begin{array}{lll}928 & 37.22 \\ 929 & 37.45\end{array}$ 92937.45 $\begin{array}{lll}930 & 37.08\end{array}$ $\begin{array}{lll}931 & 37.48\end{array}$ $932 \quad 37.11$ $933 \quad 37.06$ $934 \quad 37.35$ $935 \quad 37.48$

Vasagåd $936 \quad 37.12$ $937 \begin{array}{ll}37.29 \\ 938 & 37.35\end{array}$ $938 \begin{array}{ll}97.35 \\ 937\end{array}$ $\begin{array}{ll}938 & 37.35 \\ 939 & 37.48\end{array}$ $940 \quad 37.22$ 94137.36 $942 \quad 37.54$ $\begin{array}{llllllll}3.51 & 18.65 & 0.084 & 0.630 & 0.651 & 0.499 & 0.130 & 0.351\end{array}$ $\begin{array}{llllllll}3.69 & 18.40 & 0.084 & 0.620 & 0.675 & 0.503 & 0.110 & 0.343\end{array}$ $\begin{array}{llllllll}3.58 & 18.80 & 0.089 & 0.451 & 0.677 & 0.513 & 0.107 & 0.351\end{array}$ $\begin{array}{lllllllll}3.75 & 18.43 & 0.088 & 0.472 & 0.678 & 0.496 & 0.103 & 0.340\end{array}$ $\begin{array}{lllllllll}3.66 & 18.51 & 0.088 & 0.445 & 0.687 & 0.501 & 0.090 & 0.335\end{array}$ $\begin{array}{lllllllll}3.51 & 18.46 & 0.097 & 0.549 & 0.654 & 0.396 & 0.101 & 0.376\end{array}$ $\begin{array}{llllllll}3.60 & 18.34 & 0.096 & 0.550 & 0.613 & 0.388 & 0.116 & 0.362\end{array}$ $\begin{array}{lllllllll}3.42 & 18.43 & 0.096 & 0.549 & 0.657 & 0.397 & 0.106 & 0.350\end{array}$ $\begin{array}{llllllll}3.57 & 18.36 & 0.091 & 0.621 & 0.651 & 0.495 & 0.111 & 0.366\end{array}$ $\begin{array}{lllllllll}3.71 & 18.36 & 0.085 & 0.649 & 0.711 & 0.495 & 0.097 & 0.355\end{array}$ $\begin{array}{lllllllll}3.47 & 18.23 & 0.088 & 0.584 & 0.658 & 0.496 & 0.102 & 0.364\end{array}$ $\begin{array}{lllllllll}3.48 & 18.59 & 0.079 & 0.625 & 0.647 & 0.486 & 0.104 & 0.334\end{array}$ $\begin{array}{lllllllll}3.62 & 18.30 & 0.079 & 0.621 & 0.641 & 0.485 & 0.104 & 0.343\end{array}$ $\begin{array}{lllllllll}3.59 & 18.54 & 0.079 & 0.603 & 0.649 & 0.484 & 0.112 & 0.304\end{array}$ $\begin{array}{lllllllll}3.67 & 18.60 & 0.127 & 0.485 & 0.541 & 0.212 & 0.140 & 0.293\end{array}$ $\begin{array}{lllllllll}3.50 & 18.66 & 0.133 & 0.478 & 0.566 & 0.209 & 0.147 & 0.290\end{array}$ $\begin{array}{lllllllll}3.51 & 18.26 & 0.130 & 0.478 & 0.523 & 0.208 & 0.141 & 0.294\end{array}$ $\begin{array}{lllllllll}3.63 & 18.49 & 0.115 & 0.532 & 0.617 & 0.276 & 0.142 & 0.338\end{array}$ $\begin{array}{llllllll}3.54 & 18.52 & 0.112 & 0.536 & 0.590 & 0.288 & 0.148 & 0.358\end{array}$ $\begin{array}{llllllll}3.69 & 18.50 & 0.108 & 0.530 & 0.618 & 0.279 & 0.105 & 0.335\end{array}$ $\begin{array}{lllllllll}3.48 & 18.50 & 0.113 & 0.509 & 0.558 & 0.244 & 0.119 & 0.301\end{array}$ $\begin{array}{lllllllll}3.62 & 18.58 & 0.114 & 0.523 & 0.582 & 0.249 & 0.137 & 0.303\end{array}$ $\begin{array}{lllllllll}3.60 & 18.29 & 0.112 & 0.518 & 0.569 & 0.247 & 0.148 & 0.319\end{array}$ $\begin{array}{lllllllll}3.62 & 18.54 & 0.081 & 0.626 & 0.651 & 0.483 & 0.109 & 0.341\end{array}$ $\begin{array}{lllllllll}3.52 & 18.44 & 0.080 & 0.620 & 0.644 & 0.488 & 0.109 & 0.352\end{array}$ $\begin{array}{lllllllll}3.70 & 18.38 & 0.080 & 0.607 & 0.666 & 0.477 & 0.084 & 0.325\end{array}$ $\begin{array}{llllllll}3.67 & 18.48 & 0.080 & 0.604 & 0.634 & 0.476 & 0.117 & 0.368\end{array}$ $\begin{array}{lllllllll}3.58 & 18.30 & 0.082 & 0.601 & 0.654 & 0.481 & 0.104 & 0.356\end{array}$ $\begin{array}{lllllllll}3.59 & 18.62 & 0.078 & 0.609 & 0.598 & 0.476 & 0.101 & 0.339\end{array}$ $\begin{array}{lllllllll}3.62 & 18.59 & 0.089 & 0.607 & 0.673 & 0.494 & 0.133 & 0.287\end{array}$ $\begin{array}{lllllllll}0.00 & 0.08 & 0.050 & 0.072 & 0.641 & 0.071 & 0.033 & 0.014\end{array}$ $\begin{array}{lllllllll}3.70 & 18.57 & 0.088 & 0.607 & 0.687 & 0.490 & 0.136 & 0.297\end{array}$ $\begin{array}{lllllllll}3.63 & 18.53 & 0.095 & 0.546 & 0.624 & 0.387 & 0.116 & 0.363\end{array}$ $\begin{array}{llllllllll}3.63 & 18.53 & 0.095 & 0.546 & 0.624 & 0.387 & 0.116 & 0.363\end{array}$ $\begin{array}{llllllll}3.50 & 18.60 & 0.092 & 0.547 & 0.623 & 0.403 & 0.078 & 0.373\end{array}$ $\begin{array}{llllllll}3.48 & 18.62 & 0.079 & 0.585 & 0.618 & 0.483 & 0.088 & 0.326\end{array}$ $\begin{array}{lllllllll}3.46 & 18.56 & 0.079 & 0.607 & 0.623 & 0.484 & 0.102 & 0.339\end{array}$ $\begin{array}{llllllll}3.49 & 18.35 & 0.079 & 0.605 & 0.632 & 0.485 & 0.099 & 0.355\end{array}$ $\begin{array}{lllllllll}3.71 & 18.22 & 0.083 & 0.600 & 0.641 & 0.479 & 0.101 & 0.336\end{array}$ $\begin{array}{lllllllll}3.46 & 18.67 & 0.077 & 0.598 & 0.647 & 0.487 & 0.109 & 0.341\end{array}$ $\begin{array}{llllllll}3.57 & 18.66 & 0.087 & 0.622 & 0.627 & 0.489 & 0.114 & 0.346\end{array}$ - Bottom of bed

$\begin{array}{lllllllll}3.72 & 18.31 & 0.093 & 0.622 & 0.711 & 0.521 & 0.090 & 0.342\end{array}$ $\begin{array}{lllllllll}3.56 & 18.44 & 0.093 & 0.639 & 0.694 & 0.532 & 0.118 & 0.348\end{array}$ $\begin{array}{lllllllll}3.64 & 18.43 & 0.091 & 0.625 & 0.723 & 0.529 & 0.106 & 0.330\end{array}$ $\begin{array}{lllllllll}3.52 & 18.44 & 0.092 & 0.553 & 0.647 & 0.398 & 0.116 & 0.372\end{array}$ $\begin{array}{lllllllll}3.49 & 18.36 & 0.093 & 0.540 & 0.662 & 0.397 & 0.109 & 0.375\end{array}$ $\begin{array}{lllllllll}3.65 & 18.30 & 0.095 & 0.531 & 0.624 & 0.397 & 0.125 & 0.357\end{array}$ $\begin{array}{lllllllll}3.59 & 18.28 & 0.090 & 0.561 & 0.659 & 0.402 & 0.086 & 0.346\end{array}$
32.35 $\begin{array}{lllll}42.74 & 0.139 & 0.630 & 0.838\end{array}$ $\begin{array}{lllll}42.16 & 0.139 & 0.620 & 0.868\end{array}$ $\begin{array}{lllll}43.08 & 0.147 & 0.451 & 0.871\end{array}$ $\begin{array}{lllll}42.23 & 0.146 & 0.472 & 0.872\end{array}$ $\begin{array}{lllll}42.42 & 0.146 & 0.445 & 0.884\end{array}$ $\begin{array}{lllll}42.30 & 0.160 & 0.549 & 0.842\end{array}$ $\begin{array}{lllll}42.03 & 0.159 & 0.550 & 0.789\end{array}$ $\begin{array}{lllll}42.03 & 0.159 & 0.550 & 0.789\end{array}$ $\begin{array}{lllll}42.23 & 0.159 & 0.549 & 0.845\end{array}$ $\begin{array}{lllll}42.07 & 0.151 & 0.621 & 0.838 \\ 42.07 & 0.141 & 0.649 & 0.915\end{array}$ $\begin{array}{lllll}42.07 & 0.141 & 0.649 & 0.915\end{array}$ $\begin{array}{lllll}41.78 & 0.146 & 0.584 & 0.847\end{array}$ $\begin{array}{lllll}42.60 & 0.131 & 0.625 & 0.833\end{array}$ $\begin{array}{lllll}41.94 & 0.130 & 0.621 & 0.825\end{array}$ $\begin{array}{lllll}42.49 & 0.130 & 0.603 & 0.834\end{array}$ $\begin{array}{lllll}42.62 & 0.210 & 0.485 & 0.696\end{array}$ $\begin{array}{llllll}42.76 & 0.220 & 0.478 & 0.728\end{array}$ $\begin{array}{lllll}42.76 & 0.220 & 0.478 & 0.728\end{array}$ $\begin{array}{llll}41.84 & 0.216 & 0.478 & 0.673 \\ 42.37 & 0.191 & 0.532 & 0.793\end{array}$ $\begin{array}{lllll}42.37 & 0.191 & 0.532 & 0.793\end{array}$ $\begin{array}{lllll}42.44 & 0.185 & 0.536 & 0.759\end{array}$ $\begin{array}{lllll}42.39 & 0.179 & 0.530 & 0.795\end{array}$ $\begin{array}{lllll}42.39 & 0.187 & 0.509 & 0.718\end{array}$ $\begin{array}{lllll}42.58 & 0.189 & 0.523 & 0.748\end{array}$ $\begin{array}{lllll}41.91 & 0.186 & 0.518 & 0.732\end{array}$ $\begin{array}{lllll}42.49 & 0.135 & 0.626 & 0.838\end{array}$ $\begin{array}{lllll}42.26 & 0.132 & 0.620 & 0.829\end{array}$ $\begin{array}{lllll}42.12 & 0.132 & 0.607 & 0.857\end{array}$ $\begin{array}{lllll}42.35 & 0.132 & 0.604 & 0.816\end{array}$ $\begin{array}{lllll}41.94 & 0.135 & 0.601 & 0.841\end{array}$ $\begin{array}{lllll}42.67 & 0.130 & 0.609 & 0.769\end{array}$ $\begin{array}{lllll}42.60 & 0.148 & 0.607 & 0.865\end{array}$ $\begin{array}{lllll}0.18 & 0.083 & 0.072 & 0.824\end{array}$ $\begin{array}{lllll}42.55 & 0.145 & 0.607 & 0.883\end{array}$ $\begin{array}{lllll}42.46 & 0.157 & 0.546 & 0.802\end{array}$ $\begin{array}{lllll}42.49 & 0.151 & 0.538 & 0.831\end{array}$ $\begin{array}{lllll}42.62 & 0.152 & 0.547 & 0.802\end{array}$ $\begin{array}{lllll}42.67 & 0.131 & 0.585 & 0.795\end{array}$ $\begin{array}{lllll}42.53 & 0.131 & 0.607 & 0.802\end{array}$ $\begin{array}{lllll}42.05 & 0.130 & 0.605 & 0.813\end{array}$ $\begin{array}{lllll}41.75 & 0.137 & 0.600 & 0.825\end{array}$ $\begin{array}{lllll}42.78 & 0.128 & 0.598 & 0.832\end{array}$ $\begin{array}{lllll}42.76 & 0.145 & 0.622 & 0.807\end{array}$

$\begin{array}{llll}41.96 & 0.154 & 0.622 & 0.915\end{array}$ $\begin{array}{lllll}42.26 & 0.154 & 0.639 & 0.893\end{array}$ $\begin{array}{lllll}42.23 & 0.150 & 0.625 & 0.930\end{array}$ $\begin{array}{lllll}42.26 & 0.152 & 0.553 & 0.832\end{array}$ $\begin{array}{lllll}42.07 & 0.153 & 0.540 & 0.852\end{array}$ $\begin{array}{lllll}41.94 & 0.157 & 0.531 & 0.803\end{array}$ $\begin{array}{lllll}41.89 & 0.149 & 0.561 & 0.848\end{array}$ $\begin{array}{lll}0.644 & 0.153 & 0.446\end{array}$ $\begin{array}{lll}0.649 & 0.129 & 0.435\end{array}$ $\begin{array}{lll}0.662 & 0.125 & 0.446\end{array}$ $\begin{array}{lll}0.640 & 0.120 & 0.432\end{array}$ $\begin{array}{lll}0.646 & 0.105 & 0.425\end{array}$ $\begin{array}{llll}0.512 & 0.119 & 0.478\end{array}$ $\begin{array}{lll}0.502 & 0.136 & 0.459\end{array}$ $\begin{array}{lll}0.512 & 0.125 & 0.445\end{array}$ $\begin{array}{llll}0.639 & 0.130 & 0.465\end{array}$ $\begin{array}{llll}0.640 & 0.113 & 0.451\end{array}$ $\begin{array}{llll}0.640 & 0.120 & 0.462\end{array}$ $\begin{array}{lll}0.627 & 0.122 & 0.424\end{array}$ $\begin{array}{llll}0.627 & 0.122 & 0.435\end{array}$ $\begin{array}{llll}0.624 & 0.131 & 0.387\end{array}$ $\begin{array}{llll}0.269 & 0.172 & 0.368\end{array}$ $\begin{array}{llll}0.268 & 0.165 & 0.373\end{array}$ $\begin{array}{llll}0.357 & 0.166 & 0.429\end{array}$ $\begin{array}{llll}0.372 & 0.174 & 0.455\end{array}$ $\begin{array}{llll}0.360 & 0.123 & 0.425\end{array}$ $\begin{array}{llll}0.315 & 0.140 & 0.383\end{array}$ $\begin{array}{llll}0.322 & 0.160 & 0.385\end{array}$ $\begin{array}{llll}0.319 & 0.173 & 0.405\end{array}$ $\begin{array}{lll}0.624 & 0.128 & 0.432\end{array}$ $\begin{array}{lll}0.630 & 0.128 & 0.447\end{array}$ $\begin{array}{llll}0.616 & 0.098 & 0.413\end{array}$ $\begin{array}{llll}0.615 & 0.137 & 0.467\end{array}$ $\begin{array}{llll}0.620 & 0.121 & 0.452\end{array}$ $\begin{array}{llll}0.615 & 0.118 & 0.431\end{array}$ $\begin{array}{llll}0.637 & 0.156 & 0.364\end{array}$ $\begin{array}{lll}0.092 & 0.038 & 0.017\end{array}$ $\begin{array}{llll}0.633 & 0.159 & 0.377\end{array}$ $\begin{array}{llll}0.522 & 0.122 & 0.460\end{array}$ $\begin{array}{llll}0.623 & 0.103 & 0.414\end{array}$ $\begin{array}{llll}0.625 & 0.120 & 0.431\end{array}$ $\begin{array}{lll}0.626 & 0.115 & 0.451\end{array}$ $\begin{array}{llll}0.619 & 0.118 & 0.427\end{array}$ $\begin{array}{lll}0.628 & 0.128 & 0.433 \\ 0.632 & 0.134 & 0.440\end{array}$

$\begin{array}{llll}0.673 & 0.105 & 0.434\end{array}$ $\begin{array}{llll}0.687 & 0.138 & 0.442\end{array}$ $\begin{array}{lll}0.682 & 0.124 & 0.419\end{array}$ $\begin{array}{llll}0.514 & 0.136 & 0.472\end{array}$ $\begin{array}{llll}0.513 & 0.128 & 0.476\end{array}$ $\begin{array}{lll}0.513 & 0.128 & 0.476 \\ 0.519 & 0.146 & 0.454\end{array}$
101.4

00.5

101.6

101.5

101.1

100.9

00.3

100.6

100.3

101.0

100.8

101.2

100.3

00.6

101.2

101.3

101.5

100.8

101.0

100.7

100.8

101.1

100.7

101.4

101.1

2.1

101.3

100.8

101.1

101.2

100.2

100.1

101.

100.5

101.0

101.1

100.9

100.3 
$943 \quad 37.45$ $944 \quad 37.51$ 94537.06 $946 \quad 37.08$ $947 \quad 36.72$ $948 \quad 37.29$ 94037.29 $\begin{array}{lll}949 & 37.11 \\ 950 & 37.26\end{array}$ $\begin{array}{lll}950 & 37.26\end{array}$ 95137.08 $\begin{array}{lll}952 & 37.23 \\ 953 & 37.87\end{array}$ $953 \quad 37.87$ $954 \quad 37.28$ $955 \quad 37.38$ $956 \quad 36.15$ $957 \quad 37.20$ 95836.87 95936.66 $960 \quad 37.62$ 96137.40 $\begin{array}{lll}962 & 37.17\end{array}$ $963 \quad 37.30$ $964 \quad 37.69$ $\begin{array}{ll}965 & 37.29\end{array}$ $966 \quad 37.45$ 96637.45 96737.33 $\begin{array}{lll}968 & 37.61\end{array}$ $\begin{array}{lll}969 & 37.37\end{array}$ $\begin{array}{lll}970 & 37.30\end{array}$ 97137.21 $972 \quad 37.35$ $973 \quad 37.11$ $974 \quad 37.20$ $975 \quad 37.39$ 97637.14 97737.30 $978 \quad 37.62$ 97937.00 $980 \quad 37.69$ $981 \quad 37.34$ $\begin{array}{ll}982 & 36.99\end{array}$ $983 \quad 37.24$ $984 \quad 36.27$ $\begin{array}{lll}985 & 37.45\end{array}$ $\begin{array}{lll}986 & 37.57\end{array}$ $988 \quad 36.83$ 98936.88 $990 \quad 37.05$ 99137.04 $\begin{array}{llllllll}3.58 & 18.72 & 0.093 & 0.561 & 0.627 & 0.400 & 0.116 & 0.351\end{array}$ $\begin{array}{llllllll}3.50 & 18.61 & 0.093 & 0.561 & 0.652 & 0.401 & 0.110 & 0.359\end{array}$ $\begin{array}{llllllll}3.54 & 18.60 & 0.097 & 0.629 & 0.716 & 0.532 & 0.108 & 0.363\end{array}$ $\begin{array}{lllllllll}3.54 & 18.61 & 0.093 & 0.635 & 0.744 & 0.532 & 0.101 & 0.377\end{array}$ $\begin{array}{lllllllll}3.60 & 18.07 & 0.094 & 0.648 & 0.698 & 0.533 & 0.115 & 0.348\end{array}$ $\begin{array}{lllllllll}3.60 & 18.28 & 0.095 & 0.528 & 0.654 & 0.393 & 0.101 & 0.341\end{array}$ $\begin{array}{lllllllll}3.63 & 18.46 & 0.094 & 0.528 & 0.619 & 0.392 & 0.103 & 0.362\end{array}$ $\begin{array}{llllllll}3.66 & 18.50 & 0.091 & 0.543 & 0.618 & 0.392 & 0.108 & 0.347 \\ 3.46 & 18.48 & 0.101 & 0.456 & 0.646 & 0.355 & 0.108 & 0.369\end{array}$ $\begin{array}{llllllll}3.46 & 18.48 & 0.101 & 0.456 & 0.646 & 0.355 & 0.108 & 0.369 \\ 3.58 & 18.49 & 0.106 & 0.460 & 0.673 & 0.352 & 0.099 & 0.362\end{array}$ $\begin{array}{lllllllll}3.59 & 18.38 & 0.072 & 0.341 & 0.471 & 0.258 & 0.118 & 0.341\end{array}$ $\begin{array}{llllllll}3.46 & 18.82 & 0.089 & 0.620 & 0.694 & 0.514 & 0.108 & 0.361\end{array}$ $\begin{array}{llllllll}3.68 & 18.48 & 0.089 & 0.634 & 0.691 & 0.509 & 0.116 & 0.367\end{array}$ $\begin{array}{lllllllll}3.35 & 17.58 & 0.089 & 0.616 & 0.693 & 0.510 & 0.121 & 0.378\end{array}$ $\begin{array}{llllllll}3.60 & 18.54 & 0.081 & 0.617 & 0.629 & 0.488 & 0.111 & 0.371\end{array}$ $\begin{array}{lllllllll}3.65 & 18.45 & 0.079 & 0.615 & 0.639 & 0.484 & 0.109 & 0.379\end{array}$ $\begin{array}{llllllll}3.52 & 18.01 & 0.082 & 0.613 & 0.626 & 0.487 & 0.111 & 0.386\end{array}$ $\begin{array}{llllllll}3.70 & 18.28 & 0.149 & 0.457 & 0.581 & 0.202 & 0.147 & 0.270\end{array}$ $\begin{array}{lllllllll}3.63 & 18.77 & 0.150 & 0.470 & 0.570 & 0.204 & 0.172 & 0.275\end{array}$ $\begin{array}{llllllll}3.52 & 18.61 & 0.145 & 0.458 & 0.589 & 0.204 & 0.140 & 0.271\end{array}$ $\begin{array}{lllllllll}3.58 & 18.38 & 0.117 & 0.521 & 0.581 & 0.252 & 0.134 & 0.313\end{array}$ $\begin{array}{lllllllll}3.56 & 18.89 & 0.117 & 0.515 & 0.603 & 0.247 & 0.121 & 0.308\end{array}$ $\begin{array}{lllllllll}3.51 & 18.53 & 0.115 & 0.504 & 0.580 & 0.255 & 0.134 & 0.313\end{array}$ $\begin{array}{lllllllll}3.55 & 18.33 & 0.142 & 0.469 & 0.564 & 0.196 & 0.150 & 0.263\end{array}$ $\begin{array}{lllllllll}3.62 & 18.38 & 0.146 & 0.452 & 0.543 & 0.198 & 0.156 & 0.278\end{array}$ $\begin{array}{llllllll}3.56 & 18.39 & 0.143 & 0.466 & 0.564 & 0.202 & 0.156 & 0.274\end{array}$ $\begin{array}{lllllllll}3.71 & 18.95 & 0.093 & 0.541 & 0.629 & 0.399 & 0.135 & 0.352\end{array}$ $\begin{array}{llllllll}3.53 & 18.57 & 0.095 & 0.533 & 0.626 & 0.403 & 0.113 & 0.347\end{array}$ $\begin{array}{llllllll}3.61 & 18.76 & 0.092 & 0.549 & 0.621 & 0.400 & 0.104 & 0.359\end{array}$ $\begin{array}{lllllllll}3.54 & 18.59 & 0.084 & 0.631 & 0.672 & 0.499 & 0.078 & 0.321\end{array}$ $\begin{array}{lllllllll}3.62 & 18.55 & 0.087 & 0.613 & 0.634 & 0.500 & 0.096 & 0.318\end{array}$ $\begin{array}{lllllllll}3.60 & 18.61 & 0.082 & 0.613 & 0.660 & 0.487 & 0.085 & 0.322\end{array}$ $\begin{array}{lllllllll}3.64 & 18.47 & 0.093 & 0.547 & 0.616 & 0.379 & 0.108 & 0.339\end{array}$ $\begin{array}{lllllllll}3.61 & 18.65 & 0.089 & 0.528 & 0.632 & 0.384 & 0.077 & 0.298\end{array}$ $\begin{array}{lllllllll}3.65 & 18.68 & 0.089 & 0.522 & 0.624 & 0.377 & 0.098 & 0.337\end{array}$ $\begin{array}{llllllll}3.59 & 18.45 & 0.118 & 0.491 & 0.593 & 0.235 & 0.127 & 0.328\end{array}$ $\begin{array}{llllllll}3.69 & 18.60 & 0.122 & 0.472 & 0.595 & 0.239 & 0.130 & 0.357\end{array}$ $\begin{array}{lllllllll}3.68 & 18.40 & 0.121 & 0.497 & 0.614 & 0.257 & 0.139 & 0.365\end{array}$ $\begin{array}{lllllllll}3.54 & 18.40 & 0.089 & 0.596 & 0.657 & 0.514 & 0.089 & 0.331\end{array}$ $\begin{array}{lllllllll}3.71 & 18.66 & 0.088 & 0.597 & 0.678 & 0.497 & 0.095 & 0.333\end{array}$ $\begin{array}{lllllllll}3.63 & 18.65 & 0.088 & 0.602 & 0.668 & 0.516 & 0.100 & 0.320\end{array}$ $\begin{array}{lllllllll}3.46 & 17.98 & 0.142 & 0.476 & 0.578 & 0.204 & 0.146 & 0.271\end{array}$ $\begin{array}{lllllllll}3.67 & 18.64 & 0.141 & 0.477 & 0.556 & 0.206 & 0.149 & 0.287\end{array}$ $\begin{array}{llllllll}3.61 & 18.65 & 0.147 & 0.467 & 0.568 & 0.208 & 0.149 & 0.264\end{array}$ $\begin{array}{llllllll}3.55 & 18.71 & 0.100 & 0.555 & 0.646 & 0.339 & 0.144 & 0.465\end{array}$ $\begin{array}{llllllll}3.46 & 18.30 & 0.097 & 0.560 & 0.652 & 0.351 & 0.173 & 0.505\end{array}$ $\begin{array}{lllllllll}3.60 & 18.34 & 0.101 & 0.546 & 0.641 & 0.343 & 0.103 & 0.431\end{array}$ $\begin{array}{lllllllll}3.48 & 18.19 & 0.086 & 0.618 & 0.686 & 0.505 & 0.089 & 0.332\end{array}$
$2.51 \quad 3.50$

51.88

$51.91 \quad 3.54$

$51.41 \quad 3.60$

51.95

$51.91 \quad 3.46$

$2.12 \quad 3.58$

$53.02 \quad 3.59$

$52.19 \quad 3.46$

$\begin{array}{lll}52.33 & 3.68 \\ 50.61 & 3.35 \\ 52.08 & 3.60\end{array}$

$\begin{array}{ll}52.08 & 3.60\end{array}$

$51.62 \quad 3.65$

$51.32 \quad 3.52$

$\begin{array}{ll}52.67 & 3.70\end{array}$

$52.36 \quad 3.63$

$52.04 \quad 3.52$

$52.22 \quad 3.58$

$52.77 \quad 3.56$

$52.21 \quad 3.51$

$52.43 \quad 3.55$

$52.26 \quad 3.62$

$52.65 \quad 3.56$

$52.32 \quad 3.71$

$52.22 \quad 3.53$

$52.09 \quad 3.6$

$52.29 \quad 3.54$

$\begin{array}{ll}51.95 & 3.62 \\ 52.08 & 3.60 \\ 52.35 & 3.64\end{array}$

$52.08 \quad 3.60$

$52.00 \quad 3.61$

$52.22 \quad 3.65$

$52.67 \quad 3.59$

$51.80 \quad 3.69$

$52.77 \quad 3.68$

$\begin{array}{ll}52.28 & 3.54 \\ 51.79 & 3.71\end{array}$

$52.14 \quad 3.63$

$50.78 \quad 3.46$

$52.43 \quad 3.67$

$\begin{array}{ll}52.60 & 3.61\end{array}$

$51.80 \quad 3.55$

$51.56 \quad 3.46$

$51.63 \quad 3.60$

$51.87 \quad 3.48$

$51.86 \quad 3.53$ $\begin{array}{lllllll}42.90 & 0.155 & 0.561 & 0.806 & 0.516 & 0.135 & 0.446\end{array}$ $\begin{array}{llllllll}42.65 & 0.154 & 0.561 & 0.839 & 0.518 & 0.129 & 0.456\end{array}$ $\begin{array}{llllllll}42.62 & 0.161 & 0.629 & 0.921 & 0.687 & 0.126 & 0.461\end{array}$ $\begin{array}{llllllll}42.65 & 0.154 & 0.635 & 0.957 & 0.687 & 0.118 & 0.479\end{array}$ $\begin{array}{llllllll}41.41 & 0.157 & 0.648 & 0.898 & 0.688 & 0.135 & 0.441\end{array}$ $\begin{array}{llllllll}41.89 & 0.157 & 0.528 & 0.841 & 0.508 & 0.118 & 0.433\end{array}$ $\begin{array}{llllllll}4.30 & 0.155 & 0.528 & 0.797 & 0.506 & 0.121 & 0.460\end{array}$ $\begin{array}{lllllllll}41.89 & 0.157 & 0.528 & 0.841 & 0.508 & 0.118 & 0.433\end{array}$ $\begin{array}{llllllll}42.30 & 0.155 & 0.528 & 0.797 & 0.506 & 0.121 & 0.460 \\ 4.35 & 0.151 & 0.543 & 0.796 & 0.506 & 0.126 & 0.441\end{array}$ $\begin{array}{lllllllll}42.39 & 0.151 & 0.543 & 0.796 & 0.506 & 0.126 & 0.441\end{array}$ $\begin{array}{llllllll}42.37 & 0.175 & 0.460 & 0.866 & 0.454 & 0.116 & 0.459\end{array}$ $\begin{array}{llllllll}42.12 & 0.119 & 0.341 & 0.606 & 0.333 & 0.138 & 0.433\end{array}$ $\begin{array}{llllllll}43.13 & 0.148 & 0.620 & 0.892 & 0.663 & 0.126 & 0.458\end{array}$ $\begin{array}{llllllll}42.35 & 0.148 & 0.634 & 0.889 & 0.657 & 0.136 & 0.466\end{array}$ $\begin{array}{llllllll}40.29 & 0.148 & 0.616 & 0.892 & 0.659 & 0.141 & 0.480\end{array}$ $\begin{array}{llllllll}42.49 & 0.134 & 0.617 & 0.809 & 0.629 & 0.130 & 0.471\end{array}$ $\begin{array}{lllllll}42.28 & 0.130 & 0.615 & 0.823 & 0.625 & 0.128 & 0.481\end{array}$ $\begin{array}{llllllll}42.28 & 0.130 & 0.615 & 0.823 & 0.625 & 0.128 & 0.481\end{array}$ $\begin{array}{llllllll}41.89 & 0.136 & 0.613 & 0.806 & 0.629 & 0.130 & 0.490\end{array}$ $\begin{array}{llllllll}43.01 & 0.248 & 0.470 & 0.733 & 0.263 & 0.202 & 0.349\end{array}$ $\begin{array}{lllllll}42.65 & 0.241 & 0.458 & 0.758 & 0.263 & 0.163 & 0.344\end{array}$ $\begin{array}{llllllll}42.12 & 0.194 & 0.521 & 0.747 & 0.325 & 0.156 & 0.398\end{array}$ $\begin{array}{llllllll}43.29 & 0.193 & 0.515 & 0.775 & 0.319 & 0.141 & 0.391\end{array}$ $\begin{array}{llllllll}42.46 & 0.191 & 0.504 & 0.746 & 0.330 & 0.157 & 0.397\end{array}$ $\begin{array}{lllllll}42.00 & 0.235 & 0.469 & 0.726 & 0.253 & 0.176 & 0.334\end{array}$ $\begin{array}{lllllll}42.12 & 0.242 & 0.452 & 0.698 & 0.256 & 0.183 & 0.354\end{array}$ $\begin{array}{llllllll}42.14 & 0.237 & 0.466 & 0.726 & 0.260 & 0.183 & 0.348\end{array}$ $\begin{array}{llllllll}43.43 & 0.155 & 0.541 & 0.809 & 0.515 & 0.158 & 0.447\end{array}$ $\begin{array}{llllllll}42.55 & 0.158 & 0.533 & 0.805 & 0.521 & 0.132 & 0.441\end{array}$ $\begin{array}{llllllll}42.99 & 0.152 & 0.549 & 0.799 & 0.517 & 0.122 & 0.456\end{array}$ $\begin{array}{llllllll}42.60 & 0.139 & 0.631 & 0.865 & 0.645 & 0.091 & 0.407\end{array}$ $\begin{array}{llllllll}42.51 & 0.145 & 0.613 & 0.815 & 0.646 & 0.112 & 0.404\end{array}$ $\begin{array}{llllllll}42.65 & 0.136 & 0.613 & 0.849 & 0.629 & 0.099 & 0.409\end{array}$ $\begin{array}{llllllll}42.35 & 0.136 & 0.613 & 0.849 & 0.629 & 0.099 & 0.409\end{array}$ $\begin{array}{lllllllll}42.74 & 0.147 & 0.528 & 0.813 & 0.495 & 0.091 & 0.378\end{array}$ $\begin{array}{lllllllll}42.81 & 0.147 & 0.522 & 0.803 & 0.487 & 0.114 & 0.428\end{array}$ $\begin{array}{llllllll}42.28 & 0.196 & 0.491 & 0.763 & 0.304 & 0.148 & 0.417\end{array}$ $\begin{array}{llllllll}42.62 & 0.201 & 0.472 & 0.765 & 0.308 & 0.153 & 0.453\end{array}$ $\begin{array}{llllllll}42.16 & 0.200 & 0.497 & 0.790 & 0.332 & 0.163 & 0.463\end{array}$ $\begin{array}{llllllll}42.16 & 0.147 & 0.596 & 0.846 & 0.664 & 0.104 & 0.420\end{array}$ $\begin{array}{llllllll}42.76 & 0.145 & 0.597 & 0.872 & 0.642 & 0.112 & 0.423\end{array}$ $\begin{array}{llllllll}42.74 & 0.145 & 0.602 & 0.860 & 0.666 & 0.117 & 0.406\end{array}$ $\begin{array}{llllllll}41.20 & 0.236 & 0.476 & 0.744 & 0.264 & 0.171 & 0.344\end{array}$ $\begin{array}{llllllll}42.71 & 0.234 & 0.477 & 0.716 & 0.266 & 0.174 & 0.364\end{array}$ $\begin{array}{llllllll}42.74 & 0.244 & 0.467 & 0.731 & 0.268 & 0.174 & 0.335\end{array}$ $\begin{array}{llllllll}42.88 & 0.165 & 0.555 & 0.831 & 0.438 & 0.169 & 0.591\end{array}$ $\begin{array}{llllllll}41.94 & 0.161 & 0.560 & 0.839 & 0.454 & 0.203 & 0.641\end{array}$ $\begin{array}{llllllll}42.03 & 0.167 & 0.546 & 0.825 & 0.443 & 0.121 & 0.547\end{array}$ $\begin{array}{llllllll}41.68 & 0.143 & 0.618 & 0.883 & 0.652 & 0.105 & 0.421\end{array}$ $\begin{array}{llllllll}42.14 & 0.149 & 0.594 & 0.828 & 0.637 & 0.104 & 0.441\end{array}$
101.5

01.3

101.0

101.1

99.4

100.3

100.5

100.8

100.2

100.7

01.7

101.3

97.2

101.0

100.3

98.9

100.5

100.4

100.3

101.9

100.5

100.2

102.1

100.9

101.3

101.2

100.8

101.1

100.9

101.2

100.9

100.5

101.1

01.8

101.3

97.7

101.0

101.2

101.0

99.8

99.9

90. 
99237.08

99336.81

$994 \quad 37.25$

$\begin{array}{lllllllll}3.66 & 18.46 & 0.087 & 0.601 & 0.675 & 0.503 & 0.104 & 0.324\end{array}$ $\begin{array}{llllllll}3.53 & 18.39 & 0.090 & 0.556 & 0.625 & 0.398 & 0.116 & 0.380\end{array}$ $\begin{array}{lllllllll}3.69 & 18.41 & 0.093 & 0.555 & 0.628 & 0.405 & 0.108 & 0.348\end{array}$

$\begin{array}{lllllllll}3.51 & 18.25 & 0.092 & 0.527 & 0.604 & 0.400 & 0.127 & 0.363\end{array}$

$\mathrm{P}_{2} \mathrm{O}_{5}$

$\mathrm{Ca}, \mathrm{P}$, and $\mathrm{F}$ were not analyzed.

Vollen, Norway Kinnekulle

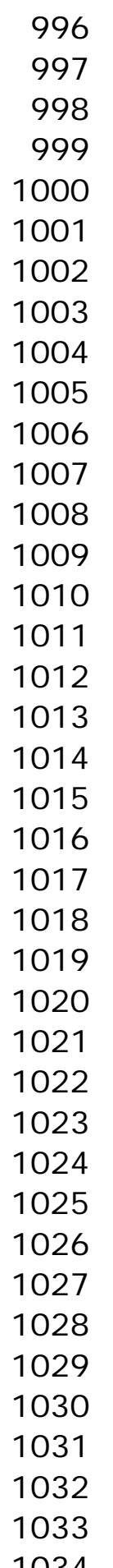

$$
\mathrm{Mg} \mathrm{Cl} \mathrm{Fe} \quad \mathrm{Mn} \mathrm{Ce} Y
$$

$\begin{array}{llllll}0.107 & 0.592 & 0.643 & 0.429 & 0.119 & 0.408\end{array}$ $\begin{array}{lllllll}0.092 & 0.559 & 0.690 & 0.438 & 0.112 & 0.393\end{array}$ $\begin{array}{lllllll}0.089 & 0.565 & 0.645 & 0.436 & 0.107 & 0.399\end{array}$ $\begin{array}{llllll}0.080 & 0.598 & 0.695 & 0.546 & 0.095 & 0.333\end{array}$ $\begin{array}{llllll}0.084 & 0.608 & 0.654 & 0.533 & 0.115 & 0.339\end{array}$ $\begin{array}{lllllll}0.080 & 0.631 & 0.681 & 0.539 & 0.086 & 0.328\end{array}$ $\begin{array}{llllll}0.092 & 0.497 & 0.647 & 0.433 & 0.090 & 0.380\end{array}$ $\begin{array}{lllllll}0.102 & 0.508 & 0.690 & 0.448 & 0.110 & 0.426\end{array}$ $\begin{array}{lllllll}0.093 & 0.543 & 0.656 & 0.445 & 0.122 & 0.428\end{array}$ $\begin{array}{lllllll}0.089 & 0.654 & 0.731 & 0.559 & 0.111 & 0.350\end{array}$ $\begin{array}{llllll}0.090 & 0.668 & 0.708 & 0.558 & 0.131 & 0.357\end{array}$ $\begin{array}{lllllll}0.093 & 0.663 & 0.742 & 0.566 & 0.119 & 0.366\end{array}$ $\begin{array}{lllllll}0.093 & 0.568 & 0.660 & 0.423 & 0.120 & 0.427\end{array}$ $\begin{array}{lllllll}0.088 & 0.591 & 0.685 & 0.438 & 0.098 & 0.407\end{array}$ $\begin{array}{lllllll}0.088 & 0.591 & 0.685 & 0.438 & 0.098 & 0.407\end{array}$ $\begin{array}{llllllll}0.099 & 0.726 & 0.647 & 0.444 & 0.111 & 0.424\end{array}$ $\begin{array}{lllllll}0.096 & 0.477 & 0.755 & 0.580 & 0.117 & 0.367\end{array}$ $\begin{array}{lllllll}0.090 & 0.463 & 0.704 & 0.561 & 0.110 & 0.380\end{array}$ $\begin{array}{lllllll}0.096 & 0.441 & 0.711 & 0.556 & 0.109 & 0.369\end{array}$ $\begin{array}{lllllll}0.125 & 0.289 & 0.567 & 0.207 & 0.135 & 0.308\end{array}$ $\begin{array}{lllllll}0.141 & 0.289 & 0.536 & 0.225 & 0.138 & 0.304\end{array}$ $\begin{array}{llllll}0.136 & 0.311 & 0.555 & 0.223 & 0.152 & 0.305\end{array}$ $\begin{array}{lllllll}0.082 & 0.658 & 0.717 & 0.551 & 0.107 & 0.371\end{array}$ $\begin{array}{lllllll}0.083 & 0.651 & 0.670 & 0.561 & 0.099 & 0.379\end{array}$ $\begin{array}{lllllll}0.083 & 0.651 & 0.670 & 0.561 & 0.099 & 0.379 \\ 0.083 & 0.672 & 0.715 & 0.555 & 0.088 & 0.350\end{array}$ $\begin{array}{llllll}0.083 & 0.672 & 0.715 & 0.555 & 0.088 & 0.350 \\ 0.089 & 0.683 & 0.709 & 0.562 & 0.098 & 0.390\end{array}$ $\begin{array}{lllllll}0.089 & 0.683 & 0.709 & 0.562 & 0.098 & 0.390 \\ 0.083 & 0.652 & 0.744 & 0.544 & 0.112 & 0.374\end{array}$ $\begin{array}{lllllll}0.083 & 0.652 & 0.744 & 0.544 & 0.112 & 0.374 \\ 0.084 & 0.622 & 0.748 & 0.574 & 0.096 & 0.376\end{array}$ $\begin{array}{lllllll}0.064 & 0.597 & 0.647 & 0.485 & 0.092 & 0.327\end{array}$ $\begin{array}{lllllll}0.084 & 0.653 & 0.668 & 0.554 & 0.102 & 0.352\end{array}$ $\begin{array}{lllllll}0.084 & 0.634 & 0.714 & 0.558 & 0.119 & 0.353\end{array}$ $\begin{array}{lllllll}0.087 & 0.688 & 0.719 & 0.554 & 0.148 & 0.418\end{array}$ $\begin{array}{lllllllll}0.087 & 0.688 & 0.719 & 0.554 & 0.148 & 0.418\end{array}$ $\begin{array}{lllllllll}0.084 & 0.683 & 0.747 & 0.564 & 0.091 & 0.390\end{array}$ $\begin{array}{lllllll}0.085 & 0.654 & 0.703 & 0.544 & 0.117 & 0.388 \\ 0.097 & 0.465 & 0.740 & 0.583 & 0.101 & 0.378\end{array}$ $\begin{array}{lllllll}0.097 & 0.465 & 0.740 & 0.583 & 0.101 & 0.378\end{array}$ $\begin{array}{lllllll}0.096 & 0.437 & 0.724 & 0.579 & 0.103 & 0.382\end{array}$ $\begin{array}{llllll}0.096 & 0.469 & 0.739 & 0.582 & 0.092 & 0.372\end{array}$ $\begin{array}{lllllll}0.089 & 0.636 & 0.674 & 0.536 & 0.094 & 0.347\end{array}$ $\begin{array}{lllllll}0.086 & 0.645 & 0.709 & 0.542 & 0.110 & 0.348\end{array}$ $\begin{array}{lllllll}0.084 & 0.630 & 0.665 & 0.534 & 0.102 & 0.358\end{array}$ 
$\begin{array}{lllllll}0.083 & 0.666 & 0.678 & 0.534 & 0.111 & 0.338\end{array}$ $\begin{array}{llllll}0.084 & 0.657 & 0.659 & 0.542 & 0.113 & 0.355\end{array}$ $\begin{array}{lllllll}0.078 & 0.650 & 0.675 & 0.535 & 0.091 & 0.337\end{array}$ $\begin{array}{lllllll}0.078 & 0.650 & 0.675 & 0.535 & 0.091 & 0.337 \\ 0.081 & 0.607 & 0.660 & 0.550 & 0.121 & 0.363\end{array}$ $\begin{array}{lllllll}0.081 & 0.607 & 0.660 & 0.550 & 0.121 & 0.363\end{array}$ $\begin{array}{lllllll}0.081 & 0.567 & 0.666 & 0.524 & 0.118 & 0.360\end{array}$ $\begin{array}{lllllll}0.091 & 0.653 & 0.685 & 0.534 & 0.134 & 0.414\end{array}$ $\begin{array}{llllll}0.090 & 0.626 & 0.725 & 0.548 & 0.143 & 0.427\end{array}$ $\begin{array}{lllllll}0.089 & 0.659 & 0.708 & 0.548 & 0.135 & 0.414\end{array}$ $\begin{array}{lllllll}0.078 & 0.638 & 0.736 & 0.551 & 0.087 & 0.334\end{array}$ $\begin{array}{lllllll}0.092 & 0.702 & 0.699 & 0.546 & 0.106 & 0.342\end{array}$ $\begin{array}{llllll}0.089 & 0.618 & 0.734 & 0.553 & 0.108 & 0.335\end{array}$ $\begin{array}{lllllll}0.088 & 0.691 & 0.740 & 0.543 & 0.104 & 0.397\end{array}$ $\begin{array}{lllllll}0.088 & 0.691 & 0.740 & 0.543 & 0.104 & 0.397 \\ 0.092 & 0.664 & 0.705 & 0.542 & 0.111 & 0.398\end{array}$ $\begin{array}{lllllll}0.092 & 0.664 & 0.705 & 0.542 & 0.111 & 0.398\end{array}$ $\begin{array}{lllllll}0.093 & 0.664 & 0.764 & 0.550 & 0.115 & 0.412\end{array}$ $\begin{array}{lllllll}0.082 & 0.657 & 0.627 & 0.521 & 0.102 & 0.354\end{array}$ $\begin{array}{lllllll}0.081 & 0.649 & 0.677 & 0.504 & 0.088 & 0.366\end{array}$ $\begin{array}{llllll}0.079 & 0.632 & 0.646 & 0.506 & 0.118 & 0.337\end{array}$ $\begin{array}{llllll}0.085 & 0.678 & 0.727 & 0.542 & 0.105 & 0.427\end{array}$ $\begin{array}{lllllll}0.087 & 0.668 & 0.697 & 0.540 & 0.120 & 0.411\end{array}$ $\begin{array}{lllllll}0.089 & 0.656 & 0.730 & 0.542 & 0.093 & 0.428\end{array}$ $\begin{array}{lllllll}0.084 & 0.668 & 0.684 & 0.535 & 0.093 & 0.355\end{array}$ $\begin{array}{lllllll}0.084 & 0.668 & 0.684 & 0.535 & 0.093 & 0.355 \\ 0.088 & 0.653 & 0.708 & 0.540 & 0.101 & 0.379\end{array}$ $\begin{array}{lllllll}0.088 & 0.653 & 0.708 & 0.540 & 0.101 & 0.379 \\ 0.088 & 0.636 & 0.693 & 0.528 & 0.103 & 0.384\end{array}$ $\begin{array}{lllllll}0.092 & 0.635 & 0.716 & 0.523 & 0.111 & 0.411\end{array}$ $\begin{array}{lllllll}0.089 & 0.643 & 0.677 & 0.519 & 0.124 & 0.394\end{array}$ $\begin{array}{lllllll}0.088 & 0.626 & 0.707 & 0.529 & 0.126 & 0.397\end{array}$ $\begin{array}{lllllll}0.087 & 0.599 & 0.680 & 0.517 & 0.103 & 0.356\end{array}$ $\begin{array}{lllllll}0.090 & 0.614 & 0.708 & 0.527 & 0.096 & 0.364\end{array}$ $\begin{array}{lllllll}0.085 & 0.606 & 0.669 & 0.508 & 0.101 & 0.350\end{array}$ $\begin{array}{llllllll}0.08 & 0.084 & 0.652 & 0.702 & 0.524 & 0.093 & 0.358\end{array}$ $\begin{array}{lllllll}0.084 & 0.652 & 0.702 & 0.524 & 0.093 & 0.388 \\ 0.084 & 0.643 & 0.653 & 0.514 & 0.103 & 0.374\end{array}$ $\begin{array}{lllllll}0.084 & 0.643 & 0.653 & 0.514 & 0.103 & 0.374\end{array}$ $\begin{array}{lllllll}0.083 & 0.638 & 0.675 & 0.519 & 0.094 & 0.361\end{array}$ $\begin{array}{lllllll}0.089 & 0.575 & 0.644 & 0.423 & 0.116 & 0.371\end{array}$ $\begin{array}{lllllll}0.092 & 0.542 & 0.666 & 0.416 & 0.107 & 0.387\end{array}$ $\begin{array}{lllllll}0.092 & 0.572 & 0.635 & 0.419 & 0.095 & 0.364\end{array}$ Mossen Section, Kinnekulle, Sweden (14-20)

$\begin{array}{lllllll}1074 & 0.09 & 0.60 & 0.721 & 0.547 & 0.103 & 0.375 \\ 1075 & 0.11 & 0.61 & 0.692 & 0.540 & 0.133 & 0.399 \\ 1076 & 0.10 & 0.50 & 0.623 & 0.386 & 0.148 & 0.420 \\ 1077 & 0.09 & 0.61 & 0.754 & 0.538 & 0.142 & 0.462 \\ 1078 & 0.09 & 0.54 & 0.709 & 0.533 & 0.121 & 0.409 \\ 1079 & 0.10 & 0.56 & 0.681 & 0.510 & 0.118 & 0.376 \\ 1080 & 0.14 & 0.59 & 0.690 & 0.538 & 0.109 & 0.364 \\ 1081 & 0.14 & 0.40 & 0.553 & 0.200 & 0.183 & 0.356 \\ 1082 & 0.10 & 0.48 & 0.792 & 0.599 & 0.138 & 0.397\end{array}$


Mossen Section, Kinnekulle, Sweden (20-26)

1098 1099

1100

1101

1102

1103

1105

1105

1107

108 109

1110

11112

1113

1114

1115

1116

1117

1118

1119

1120

1121

1122

1123

1125

1126

1127

1129 $\begin{array}{lllllll}0.09 & 0.59 & 0.716 & 0.526 & 0.121 & 0.365\end{array}$

$\begin{array}{lllllll}0.11 & 0.53 & 0.718 & 0.469 & 0.148 & 0.468\end{array}$

$\begin{array}{lllllll}0.10 & 0.59 & 0.709 & 0.534 & 0.128 & 0.380\end{array}$

$\begin{array}{llllll}0.09 & 0.56 & 0.677 & 0.488 & 0.112 & 0.384\end{array}$

$\begin{array}{llllll}0.10 & 0.57 & 0.712 & 0.526 & 0.127 & 0.419\end{array}$

$\begin{array}{lllllll}0.13 & 0.55 & 0.699 & 0.539 & 0.106 & 0.359\end{array}$

$\begin{array}{lllllll}0.09 & 0.60 & 0.711 & 0.543 & 0.129 & 0.381\end{array}$

$\begin{array}{lllllll}0.11 & 0.49 & 0.644 & 0.350 & 0.144 & 0.378\end{array}$

$\begin{array}{llllll}0.12 & 0.60 & 0.741 & 0.546 & 0.141 & 0.413\end{array}$

$\begin{array}{llllll}0.10 & 0.58 & 0.683 & 0.521 & 0.115 & 0.345\end{array}$ $\begin{array}{lllllll}0.08 & 0.58 & 0.659 & 0.509 & 0.121 & 0.378\end{array}$ Millbrig

$\begin{array}{llllll}0.010 & 0.202 & 0.056 & 0.079 & 0.125 & 0.089\end{array}$ $\begin{array}{lllllll}0.007 & 0.207 & 0.080 & 0.082 & 0.158 & 0.155\end{array}$ $\begin{array}{llllll}0.009 & 0.180 & 0.077 & 0.072 & 0.130 & 0.108\end{array}$ $\begin{array}{lllllll}0.007 & 0.224 & 0.084 & 0.099 & 0.085 & 0.131\end{array}$ $\begin{array}{lllllll}0.009 & 0.197 & 0.069 & 0.101 & 0.088 & 0.141\end{array}$ $\begin{array}{lllllll}0.007 & 0.210 & 0.067 & 0.094 & 0.078 & 0.138\end{array}$ $\begin{array}{lllllll}0.009 & 0.198 & 0.066 & 0.074 & 0.077 & 0.105\end{array}$ $\begin{array}{lllllll}0.009 & 0.198 & 0.066 & 0.074 & 0.077 & 0.105 \\ 0.011 & 0.206 & 0.079 & 0.081 & 0.088 & 0.102 \\ 0.011 & 0.219 & 0.072 & 0.085 & 0.093 & 0.105\end{array}$ $\begin{array}{lllllll}0.011 & 0.231 & 0.077 & 0.066 & 0.344 & 0.169\end{array}$ $\begin{array}{llllll}0.011 & 0.167 & 0.091 & 0.086 & 0.203 & 0.095\end{array}$ $\begin{array}{lllllll}0.008 & 0.188 & 0.091 & 0.076 & 0.186 & 0.086\end{array}$ $\begin{array}{lllllll}0.010 & 0.220 & 0.077 & 0.088 & 0.189 & 0.204\end{array}$ $\begin{array}{lllllll}0.009 & 0.211 & 0.084 & 0.093 & 0.150 & 0.160\end{array}$ $\begin{array}{llllllll}0.009 & 0.211 & 0.084 & 0.093 & 0.150 & 0.160\end{array}$ $\begin{array}{lllllll}0.011 & 0.210 & 0.063 & 0.086 & 0.122 & 0.148 \\ 0.180 & 0.005 & 0.831 & 0.176 & 0.209 & 0.163\end{array}$ $\begin{array}{lllllll}0.180 & 0.005 & 0.831 & 0.176 & 0.209 & 0.163 \\ 0.179 & 0.004 & 0.766 & 0.169 & 0.193 & 0.175\end{array}$ $\begin{array}{lllllll}0.179 & 0.004 & 0.766 & 0.169 & 0.193 & 0.175\end{array}$ $\begin{array}{lllllll}0.173 & 0.004 & 0.842 & 0.176 & 0.193 & 0.155\end{array}$ $\begin{array}{lllllll}0.008 & 0.187 & 0.068 & 0.076 & 0.059 & 0.071\end{array}$ $\begin{array}{lllllll}0.009 & 0.185 & 0.083 & 0.080 & 0.073 & 0.063\end{array}$ $\begin{array}{lllllll}0.009 & 0.183 & 0.060 & 0.077 & 0.047 & 0.071\end{array}$ 
$\begin{array}{lllllll}0.009 & 0.219 & 0.061 & 0.082 & 0.206 & 0.247\end{array}$ $\begin{array}{lllllll}0.008 & 0.192 & 0.073 & 0.077 & 0.196 & 0.248\end{array}$ $\begin{array}{lllllll}0.009 & 0.225 & 0.074 & 0.079 & 0.223 & 0.263\end{array}$ $\begin{array}{lllllll}0.010 & 0.196 & 0.079 & 0.077 & 0.089 & 0.115\end{array}$ $\begin{array}{llllll}0.009 & 0.210 & 0.074 & 0.082 & 0.071 & 0.113\end{array}$ $\begin{array}{lllllll}0.010 & 0.208 & 0.075 & 0.080 & 0.091 & 0.110\end{array}$ $\begin{array}{lllllll}0.010 & 0.254 & 0.071 & 0.076 & 0.138 & 0.162\end{array}$ $\begin{array}{lllllll}0.010 & 0.254 & 0.071 & 0.076 & 0.138 & 0.162 \\ 0.007 & 0.253 & 0.067 & 0.080 & 0.130 & 0.148\end{array}$ $\begin{array}{lllllll}0.007 & 0.253 & 0.067 & 0.080 & 0.130 & 0.148\end{array}$ $\begin{array}{lllllll}0.011 & 0.200 & 0.078 & 0.075 & 0.134 & 0.152\end{array}$ $\begin{array}{lllllll}0.005 & 0.187 & 0.070 & 0.079 & 0.088 & 0.102\end{array}$ $\begin{array}{llllll}0.009 & 0.182 & 0.071 & 0.083 & 0.093 & 0.104\end{array}$ $\begin{array}{lllllll}0.012 & 0.184 & 0.083 & 0.071 & 0.081 & 0.111\end{array}$ $\begin{array}{lllllll}0.011 & 0.193 & 0.078 & 0.078 & 0.118 & 0.104\end{array}$ $\begin{array}{lllllll}0.010 & 0.190 & 0.071 & 0.078 & 0.103 & 0.114\end{array}$ $\begin{array}{lllllll}0.009 & 0.171 & 0.077 & 0.079 & 0.109 & 0.128\end{array}$ $\begin{array}{llllllll}0.008 & 0.199 & 0.071 & 0.081 & 0.147 & 0.204\end{array}$ $\begin{array}{lllllll}0.008 & 0.199 & 0.071 & 0.081 & 0.147 & 0.204 \\ 0.009 & 0.224 & 0.071 & 0.090 & 0.214 & 0.256\end{array}$ $\begin{array}{lllllll}0.009 & 0.224 & 0.071 & 0.090 & 0.214 & 0.256\end{array}$ $\begin{array}{lllllll}0.010 & 0.231 & 0.098 & 0.084 & 0.190 & 0.258\end{array}$ $\begin{array}{lllllll}0.009 & 0.221 & 0.065 & 0.077 & 0.102 & 0.124\end{array}$ $\begin{array}{llllll}0.009 & 0.238 & 0.082 & 0.088 & 0.122 & 0.143\end{array}$ $\begin{array}{lllllll}0.011 & 0.202 & 0.082 & 0.084 & 0.151 & 0.196\end{array}$ $\begin{array}{lllllll}0.011 & 0.235 & 0.090 & 0.077 & 0.235 & 0.310\end{array}$ $\begin{array}{lllllll}0.008 & 0.217 & 0.073 & 0.076 & 0.290 & 0.367\end{array}$ $\begin{array}{lllllll}0.009 & 0.212 & 0.084 & 0.085 & 0.270 & 0.333\end{array}$ $\begin{array}{lllllllll}0.008 & 0.186 & 0.062 & 0.086 & 0.114 & 0.333\end{array}$ $\begin{array}{lllllll}0.008 & 0.186 & 0.062 & 0.086 & 0.114 & 0.118\end{array}$ $\begin{array}{llllll}0.006 & 0.194 & 0.081 & 0.079 & 0.101 & 0.117 \\ 0.009 & 0.195 & 0.081 & 0.081 & 0.111 & 0.117\end{array}$ State College ( 322 \#8) Millbrig

$\begin{array}{llllllll}0.006 & 0.228 & 0.046 & 0.089 & 0.124 & 0.302\end{array}$ $\begin{array}{lllllll}0.008 & 0.249 & 0.057 & 0.092 & 0.189 & 0.425\end{array}$ $\begin{array}{lllllll}0.005 & 0.275 & 0.064 & 0.109 & 0.160 & 0.367\end{array}$ $\begin{array}{llllll}0.019 & 0.412 & 0.097 & 0.080 & 0.180 & 0.069\end{array}$ $\begin{array}{lllllll}0.019 & 0.440 & 0.131 & 0.075 & 0.171 & 0.072\end{array}$ $\begin{array}{lllllll}0.020 & 0.416 & 0.117 & 0.081 & 0.188 & 0.072\end{array}$ $\begin{array}{lllllll}0.020 & 0.416 & 0.117 & 0.081 & 0.188 & 0.072 \\ 0.005 & 0.205 & 0.059 & 0.093 & 0.045 & 0.086\end{array}$ $\begin{array}{lllllll}0.005 & 0.205 & 0.059 & 0.093 & 0.045 & 0.086 \\ 0.006 & 0.206 & 0.058 & 0.111 & 0.053 & 0.097 \\ 0.005 & 0.205 & 0.075 & 0.099 & 0.059 & 0.087\end{array}$ $\begin{array}{lllllll}0.006 & 0.206 & 0.058 & 0.111 & 0.053 & 0.097 \\ 0.005 & 0.205 & 0.075 & 0.099 & 0.059 & 0.087\end{array}$ $\begin{array}{lllllll}0.005 & 0.205 & 0.075 & 0.099 & 0.059 & 0.087 \\ 0.024 & 0.299 & 0.119 & 0.042 & 0.200 & 0.110\end{array}$ $\begin{array}{llllll}0.026 & 0.282 & 0.120 & 0.043 & 0.233 & 0.108\end{array}$ $\begin{array}{lllllll}0.026 & 0.262 & 0.104 & 0.039 & 0.278 & 0.143\end{array}$ 
$\begin{array}{lllllll}0.026 & 0.266 & 0.117 & 0.037 & 0.188 & 0.101\end{array}$ $\begin{array}{lllllll}0.027 & 0.261 & 0.117 & 0.036 & 0.171 & 0.086\end{array}$ $\begin{array}{lllllll}0.029 & 0.248 & 0.125 & 0.039 & 0.155 & 0.089\end{array}$ $\begin{array}{lllllll}0.029 & 0.248 & 0.125 & 0.039 & 0.155 & 0.089 \\ 0.028 & 0.256 & 0.111 & 0.043 & 0.191 & 0.097\end{array}$ $\begin{array}{lllllll}0.028 & 0.256 & 0.111 & 0.043 & 0.191 & 0.097 \\ 0.027 & 0.238 & 0.125 & 0.041 & 0.155 & 0.086\end{array}$ $\begin{array}{lllllll}0.027 & 0.238 & 0.125 & 0.041 & 0.155 & 0.086\end{array}$ $\begin{array}{lllllll}0.016 & 0.423 & 0.107 & 0.095 & 0.311 & 0.145\end{array}$ $\begin{array}{lllllll}0.020 & 0.417 & 0.117 & 0.092 & 0.271 & 0.121\end{array}$ $\begin{array}{lllllll}0.020 & 0.411 & 0.119 & 0.089 & 0.284 & 0.144\end{array}$ $\begin{array}{llllll}0.027 & 0.287 & 0.120 & 0.047 & 0.248 & 0.140\end{array}$ $\begin{array}{lllllll}0.025 & 0.281 & 0.099 & 0.040 & 0.284 & 0.133\end{array}$ $\begin{array}{lllllll}0.024 & 0.268 & 0.125 & 0.040 & 0.279 & 0.124\end{array}$ $\begin{array}{lllllll}0.038 & 0.271 & 0.122 & 0.052 & 0.180 & 0.108\end{array}$ $\begin{array}{lllllll}0.038 & 0.271 & 0.122 & 0.052 & 0.180 & 0.108 \\ 0.036 & 0.262 & 0.146 & 0.034 & 0.139 & 0.087\end{array}$ $\begin{array}{lllllll}0.036 & 0.262 & 0.146 & 0.034 & 0.139 & 0.087 \\ 0.037 & 0.255 & 0.122 & 0.040 & 0.173 & 0.091\end{array}$ $\begin{array}{lllllll}0.037 & 0.255 & 0.122 & 0.040 & 0.173 & 0.091\end{array}$ $\begin{array}{lllllll}0.026 & 0.297 & 0.120 & 0.039 & 0.231 & 0.120\end{array}$ $\begin{array}{lllllll}0.027 & 0.282 & 0.115 & 0.039 & 0.199 & 0.105\end{array}$ $\begin{array}{llllll}0.024 & 0.267 & 0.114 & 0.044 & 0.175 & 0.094\end{array}$ $\begin{array}{lllllll}0.028 & 0.329 & 0.107 & 0.041 & 0.283 & 0.144\end{array}$ $\begin{array}{lllllll}0.025 & 0.322 & 0.123 & 0.037 & 0.232 & 0.117\end{array}$ $\begin{array}{lllllll}0.027 & 0.323 & 0.114 & 0.037 & 0.284 & 0.155\end{array}$ $\begin{array}{lllllll}0.026 & 0.259 & 0.120 & 0.042 & 0.277 & 0.142\end{array}$ $\begin{array}{lllllll}0.026 & 0.259 & 0.120 & 0.042 & 0.277 & 0.142 \\ 0.026 & 0.251 & 0.114 & 0.039 & 0.369 & 0.208\end{array}$ $\begin{array}{lllllll}0.026 & 0.251 & 0.114 & 0.039 & 0.369 & 0.208\end{array}$ $\begin{array}{lllllll}0.027 & 0.235 & 0.113 & 0.038 & 0.265 & 0.137\end{array}$ $\begin{array}{lllllll}0.028 & 0.267 & 0.119 & 0.045 & 0.159 & 0.083\end{array}$ $\begin{array}{lllllll}0.022 & 0.283 & 0.101 & 0.045 & 0.132 & 0.066\end{array}$ $\begin{array}{lllllll}0.025 & 0.280 & 0.108 & 0.041 & 0.213 & 0.085\end{array}$ $\begin{array}{lllllll}0.026 & 0.275 & 0.125 & 0.051 & 0.222 & 0.104\end{array}$ $\begin{array}{lllllll}0.027 & 0.298 & 0.115 & 0.035 & 0.220 & 0.097\end{array}$ $\begin{array}{lllllll}0.028 & 0.287 & 0.124 & 0.043 & 0.226 & 0.115\end{array}$ $\begin{array}{lllllllll}0.006 & 0.014 & 0.031 & 0.001 & 0.017 & 0.411\end{array}$ $\begin{array}{lllllll}0.006 & 0.014 & 0.031 & 0.001 & 0.017 & 0.411 \\ 0.007 & 0.009 & 0.031 & 0.000 & 0.033 & 0.654\end{array}$ $\begin{array}{lllllll}0.007 & 0.009 & 0.031 & 0.000 & 0.033 & 0.654\end{array}$ $\begin{array}{lllllll}0.002 & 0.010 & 0.000 & 0.000 & 0.007 & 0.393\end{array}$ $\begin{array}{lllllll}0.019 & 0.411 & 0.130 & 0.085 & 0.339 & 0.153\end{array}$ $\begin{array}{llllll}0.022 & 0.363 & 0.121 & 0.086 & 0.254 & 0.123\end{array}$ $\begin{array}{llllll}0.019 & 0.443 & 0.134 & 0.088 & 0.333 & 0.153\end{array}$ $\begin{array}{lllllll}0.005 & 0.263 & 0.063 & 0.108 & 0.103 & 0.149\end{array}$ $\begin{array}{lllllll}0.007 & 0.286 & 0.067 & 0.101 & 0.103 & 0.172\end{array}$ $\begin{array}{lllllll}0.005 & 0.264 & 0.058 & 0.105 & 0.112 & 0.158\end{array}$ $\begin{array}{llllllll}0.005 & 0.264 & 0.058 & 0.105 & 0.112 & 0.158\end{array}$ $\begin{array}{llllllll}0.015 & 0.365 & 0.113 & 0.070 & 0.203 & 0.091 \\ 0.017 & 0.425 & 0.117 & 0.083 & 0.204 & 0.086\end{array}$ $\begin{array}{llllllll}0.017 & 0.425 & 0.117 & 0.083 & 0.204 & 0.086\end{array}$ $\begin{array}{llllllll}0.017 & 0.432 & 0.142 & 0.101 & 0.177 & 0.087\end{array}$ $\begin{array}{lllllll}0.017 & 0.313 & 0.099 & 0.058 & 0.328 & 0.161\end{array}$ $\begin{array}{lllllll}0.016 & 0.312 & 0.104 & 0.060 & 0.246 & 0.133\end{array}$ $\begin{array}{lllllll}0.019 & 0.304 & 0.095 & 0.051 & 0.272 & 0.134\end{array}$ $\begin{array}{llllll}0.024 & 0.287 & 0.125 & 0.043 & 0.209 & 0.115\end{array}$ 
$\begin{array}{llllll}0.022 & 0.284 & 0.108 & 0.037 & 0.204 & 0.107\end{array}$ $\begin{array}{llllll}0.023 & 0.294 & 0.118 & 0.049 & 0.183 & 0.097\end{array}$ $\begin{array}{llllll}0.025 & 0.314 & 0.102 & 0.038 & 0.220 & 0.110\end{array}$ $\begin{array}{lllllll}0.022 & 0.311 & 0.122 & 0.034 & 0.186 & 0.105\end{array}$ $\begin{array}{llllll}0.025 & 0.307 & 0.096 & 0.051 & 0.203 & 0.098\end{array}$ $\begin{array}{lllllll}0.009 & 0.372 & 0.093 & 0.031 & 0.114 & 0.028\end{array}$ $\begin{array}{lllllll}0.026 & 0.310 & 0.102 & 0.049 & 0.193 & 0.087\end{array}$ $\begin{array}{lllllll}0.026 & 0.310 & 0.102 & 0.049 & 0.193 & 0.087 \\ 0.022 & 0.301 & 0.122 & 0.051 & 0.145 & 0.086\end{array}$ $\begin{array}{lllllll}0.022 & 0.301 & 0.122 & 0.051 & 0.145 & 0.086 \\ 0.026 & 0.310 & 0.106 & 0.045 & 0.226 & 0.101\end{array}$ $\begin{array}{lllllll}0.026 & 0.310 & 0.106 & 0.045 & 0.226 & 0.101\end{array}$ $\begin{array}{lllllll}0.021 & 0.280 & 0.113 & 0.043 & 0.217 & 0.104\end{array}$ $\begin{array}{lllllll}0.022 & 0.311 & 0.108 & 0.041 & 0.204 & 0.086\end{array}$ $\begin{array}{llllll}0.022 & 0.323 & 0.132 & 0.040 & 0.226 & 0.103\end{array}$ $\begin{array}{lllllll}0.025 & 0.307 & 0.103 & 0.040 & 0.207 & 0.103\end{array}$ $\begin{array}{lllllll}0.022 & 0.314 & 0.116 & 0.046 & 0.204 & 0.096\end{array}$ $\begin{array}{lllllll}0.025 & 0.293 & 0.109 & 0.049 & 0.237 & 0.114\end{array}$ $\begin{array}{lllllll}0.020 & 0.292 & 0.128 & 0.049 & 0.204 & 0.107\end{array}$ $\begin{array}{lllllll}0.020 & 0.292 & 0.128 & 0.049 & 0.204 & 0.107 \\ 0.022 & 0.281 & 0.100 & 0.041 & 0.232 & 0.106\end{array}$ $\begin{array}{lllllll}0.022 & 0.281 & 0.100 & 0.041 & 0.232 & 0.106 \\ 0.038 & 0.307 & 0.143 & 0.039 & 0.276 & 0.145\end{array}$ $\begin{array}{lllllll}0.038 & 0.307 & 0.143 & 0.039 & 0.276 & 0.145\end{array}$ $\begin{array}{lllllll}0.038 & 0.315 & 0.111 & 0.037 & 0.282 & 0.174\end{array}$ $\begin{array}{lllllll}0.037 & 0.318 & 0.131 & 0.040 & 0.283 & 0.173\end{array}$ $\begin{array}{lllllll}0.024 & 0.417 & 0.125 & 0.066 & 0.266 & 0.106\end{array}$ $\begin{array}{lllllll}0.023 & 0.425 & 0.132 & 0.066 & 0.224 & 0.083\end{array}$ $\begin{array}{llllll}0.024 & 0.430 & 0.123 & 0.065 & 0.292 & 0.114\end{array}$ $\begin{array}{lllllll}0.023 & 0.434 & 0.146 & 0.059 & 0.181 & 0.067\end{array}$ $\begin{array}{llllllll}0.026 & 0.415 & 0.134 & 0.063 & 0.234 & 0.085\end{array}$ $\begin{array}{lllllll}0.026 & 0.415 & 0.134 & 0.063 & 0.234 & 0.085\end{array}$ $\begin{array}{lllllll}0.013 & 0.171 & 0.075 & 0.074 & 0.118 & 0.115\end{array}$ $\begin{array}{lllllll}0.011 & 0.164 & 0.080 & 0.063 & 0.115 & 0.135\end{array}$ $\begin{array}{lllllll}0.011 & 0.241 & 0.064 & 0.069 & 0.133 & 0.112\end{array}$ $\begin{array}{llllll}0.012 & 0.174 & 0.079 & 0.077 & 0.098 & 0.122\end{array}$ $\begin{array}{lllllll}0.009 & 0.141 & 0.071 & 0.064 & 0.101 & 0.128\end{array}$ $\begin{array}{lllllll}0.009 & 0.157 & 0.078 & 0.069 & 0.100 & 0.115\end{array}$ $\begin{array}{llllllll}0.013 & 0.190 & 0.070 & 0.059 & 0.113 & 0.156\end{array}$ $\begin{array}{lllllll}0.013 & 0.190 & 0.070 & 0.059 & 0.113 & 0.156 \\ 0.012 & 0.194 & 0.072 & 0.073 & 0.114 & 0.144\end{array}$ $\begin{array}{lllllll}0.012 & 0.194 & 0.072 & 0.073 & 0.114 & 0.144 \\ 0.011 & 0.149 & 0.062 & 0.066 & 0.120 & 0.164\end{array}$ $\begin{array}{lllllll}0.011 & 0.149 & 0.062 & 0.066 & 0.120 & 0.164\end{array}$ $\begin{array}{llllll}0.011 & 0.158 & 0.077 & 0.069 & 0.112 & 0.117\end{array}$ $\begin{array}{lllllll}0.008 & 0.209 & 0.057 & 0.076 & 0.086 & 0.112\end{array}$ $\begin{array}{lllllll}0.010 & 0.148 & 0.085 & 0.073 & 0.101 & 0.110\end{array}$ $\begin{array}{lllllll}0.009 & 0.156 & 0.067 & 0.064 & 0.084 & 0.120\end{array}$ $\begin{array}{llllll}0.008 & 0.107 & 0.071 & 0.072 & 0.092 & 0.127\end{array}$ $\begin{array}{lllllll}0.012 & 0.156 & 0.075 & 0.069 & 0.071 & 0.084\end{array}$ $\begin{array}{llllllll}0.012 & 0.156 & 0.075 & 0.069 & 0.071 & 0.084 \\ 0.013 & 0.185 & 0.080 & 0.071 & 0.089 & 0.120\end{array}$ $\begin{array}{lllllll}0.013 & 0.185 & 0.080 & 0.071 & 0.089 & 0.120 \\ 0.011 & 0.225 & 0.065 & 0.065 & 0.088 & 0.124\end{array}$ $\begin{array}{lllllll}0.011 & 0.225 & 0.065 & 0.065 & 0.088 & 0.124 \\ 0.012 & 0.224 & 0.067 & 0.072 & 0.099 & 0.114\end{array}$ $\begin{array}{lllllll}0.012 & 0.224 & 0.067 & 0.072 & 0.099 & 0.114\end{array}$ $\begin{array}{lllllll}0.010 & 0.204 & 0.065 & 0.079 & 0.088 & 0.111\end{array}$ $\begin{array}{lllllll}0.008 & 0.161 & 0.062 & 0.091 & 0.081 & 0.103\end{array}$ $\begin{array}{llllll}0.008 & 0.154 & 0.068 & 0.080 & 0.090 & 0.102\end{array}$ $\begin{array}{llllll}0.008 & 0.179 & 0.068 & 0.082 & 0.098 & 0.120\end{array}$ 
$\begin{array}{lllllll}0.029 & 0.149 & 0.096 & 0.039 & 0.129 & 0.095\end{array}$

$\begin{array}{lllllll}0.011 & 0.190 & 0.074 & 0.075 & 0.082 & 0.112\end{array}$

$\begin{array}{lllllll}0.011 & 0.158 & 0.075 & 0.068 & 0.084 & 0.107\end{array}$ $\begin{array}{llllllll}0.011 & 0.158 & 0.075 & 0.068 & 0.084 & 0.107 \\ 0.011 & 0.197 & 0.074 & 0.071 & 0.065 & 0.102\end{array}$ $\begin{array}{lllllll}0.001 & 0.012 & 0.000 & 0.000 & 0.014 & 0.042\end{array}$ $\begin{array}{lllllll}0.001 & 0.012 & 0.000 & 0.000 & 0.014 & 0.042 \\ 0.000 & 0.000 & 0.001 & 0.002 & 0.008 & 0.051\end{array}$ $\begin{array}{llllll}0.000 & 0.000 & 0.001 & 0.002 & 0.008 & 0.051 \\ 0.001 & 0.008 & 0.000 & 0.000 & 0.017 & 0.049\end{array}$ $\begin{array}{lllllll}0.010 & 0.214 & 0.060 & 0.091 & 0.100 & 0.167\end{array}$ $\begin{array}{llllll}0.009 & 0.148 & 0.067 & 0.075 & 0.098 & 0.157\end{array}$ $\begin{array}{lllllll}0.008 & 0.211 & 0.066 & 0.085 & 0.100 & 0.169\end{array}$ $\begin{array}{lllllll}0.009 & 0.332 & 0.070 & 0.074 & 0.142 & 0.112\end{array}$ $\begin{array}{lllllll}0.008 & 0.151 & 0.074 & 0.071 & 0.078 & 0.078\end{array}$ $\begin{array}{lllllll}0.008 & 0.151 & 0.074 & 0.071 & 0.078 & 0.078\end{array}$ $\begin{array}{lllllll}0.010 & 0.183 & 0.064 & 0.063 & 0.093 & 0.081 \\ 0.010 & 0.171 & 0.064 & 0.071 & 0.102 & 0.141\end{array}$ $\begin{array}{lllllll}0.010 & 0.171 & 0.064 & 0.071 & 0.102 & 0.141\end{array}$ $\begin{array}{lllllll}0.012 & 0.182 & 0.062 & 0.069 & 0.108 & 0.141\end{array}$ $\begin{array}{lllllll}0.013 & 0.198 & 0.072 & 0.073 & 0.108 & 0.160\end{array}$ $\begin{array}{lllllll}0.011 & 0.172 & 0.067 & 0.065 & 0.111 & 0.158\end{array}$ $\begin{array}{llllll}0.009 & 0.170 & 0.077 & 0.072 & 0.131 & 0.123\end{array}$ $\begin{array}{lllllll}0.010 & 0.196 & 0.078 & 0.068 & 0.094 & 0.112\end{array}$ $\begin{array}{lllllll}0.024 & 0.151 & 0.123 & 0.043 & 0.229 & 0.142\end{array}$ $\begin{array}{llllllll}0.027 & 0.162 & 0.108 & 0.031 & 0.230 & 0.141\end{array}$ $\begin{array}{lllllll}0.027 & 0.162 & 0.108 & 0.031 & 0.230 & 0.141\end{array}$ $\begin{array}{lllllll}0.028 & 0.142 & 0.113 & 0.039 & 0.180 & 0.119 \\ 0.010 & 0.182 & 0.083 & 0.067 & 0.193 & 0.231\end{array}$ $\begin{array}{lllllll}0.010 & 0.182 & 0.083 & 0.067 & 0.193 & 0.231 \\ 0.009 & 0.168 & 0.069 & 0.068 & 0.198 & 0.284\end{array}$ $\begin{array}{lllllll}0.009 & 0.168 & 0.069 & 0.068 & 0.198 & 0.284\end{array}$ $\begin{array}{lllllll}0.009 & 0.244 & 0.072 & 0.063 & 0.220 & 0.283\end{array}$ $\begin{array}{lllllll}0.012 & 0.161 & 0.075 & 0.070 & 0.087 & 0.124\end{array}$ $\begin{array}{lllllll}0.009 & 0.164 & 0.063 & 0.062 & 0.078 & 0.106\end{array}$ $\begin{array}{lllllll}0.011 & 0.159 & 0.071 & 0.075 & 0.134 & 0.172\end{array}$

Dolly Ridge, WV Millbrig

1307 1308 1309

1310

1311

1313

1314

1315

1317

1318

1319

1320

1322 $\begin{array}{lllll}0.019 & 0.277 & 0.117 & 0.083\end{array}$

$\begin{array}{llll}0.016 & 0.281 & 0.125 & 0.078\end{array}$

$\begin{array}{llll}0.016 & 0.281 & 0.125 & 0.078 \\ 0.019 & 0.261 & 0.111 & 0.075\end{array}$

$\begin{array}{llll}0.019 & 0.261 & 0.111 & 0.075 \\ 0.012 & 0.245 & 0.078 & 0.078\end{array}$

$\begin{array}{lllll}0.010 & 0.243 & 0.092 & 0.100\end{array}$

$\begin{array}{lllll}0.012 & 0.248 & 0.089 & 0.083\end{array}$

$\begin{array}{llll}0.009 & 0.238 & 0.067 & 0.088\end{array}$

$\begin{array}{llll}0.008 & 0.223 & 0.073 & 0.103\end{array}$

$\begin{array}{lllll}0.011 & 0.228 & 0.070 & 0.107\end{array}$

$\begin{array}{lllll}0.011 & 0.248 & 0.084 & 0.091\end{array}$

$\begin{array}{lllll}0.009 & 0.252 & 0.078 & 0.094\end{array}$

$\begin{array}{lllll}0.009 & 0.230 & 0.072 & 0.099\end{array}$

$\begin{array}{lllll}0.012 & 0.229 & 0.079 & 0.087\end{array}$

$\begin{array}{lllll}0.011 & 0.219 & 0.092 & 0.075\end{array}$

$\begin{array}{llll}0.012 & 0.232 & 0.087 & 0.081\end{array}$

$\begin{array}{llll}0.035 & 0.300 & 0.122 & 0.078\end{array}$ 
$\begin{array}{lllll}0.011 & 0.253 & 0.072 & 0.074\end{array}$

$\begin{array}{lllll}0.011 & 0.268 & 0.094 & 0.065\end{array}$

$\begin{array}{lllll}0.010 & 0.245 & 0.084 & 0.082\end{array}$

$\begin{array}{llll}0.013 & 0.253 & 0.084 & 0.085\end{array}$

$\begin{array}{llll}0.009 & 0.237 & 0.081 & 0.096\end{array}$

$\begin{array}{llll}0.010 & 0.225 & 0.094 & 0.089\end{array}$

$\begin{array}{llll}0.011 & 0.243 & 0.079 & 0.102\end{array}$

$\begin{array}{lllll}0.007 & 0.207 & 0.034 & 0.091\end{array}$

$\begin{array}{lllll}0.008 & 0.225 & 0.073 & 0.074\end{array}$

$\begin{array}{lllll}0.010 & 0.227 & 0.029 & 0.092\end{array}$

$\begin{array}{lllll}0.012 & 0.150 & 0.110 & 0.069\end{array}$

$\begin{array}{llll}0.014 & 0.166 & 0.058 & 0.096\end{array}$

$\begin{array}{lllll}0.011 & 0.162 & 0.103 & 0.019\end{array}$

$\begin{array}{lllll}0.013 & 0.267 & 0.091 & 0.075\end{array}$

$\begin{array}{llll}0.011 & 0.244 & 0.074 & 0.091\end{array}$

$\begin{array}{llll}0.011 & 0.248 & 0.085 & 0.082\end{array}$

$\begin{array}{lllll}0.020 & 0.261 & 0.127 & 0.064\end{array}$

$\begin{array}{lllll}0.021 & 0.254 & 0.134 & 0.072\end{array}$

$\begin{array}{lllll}0.017 & 0.257 & 0.113 & 0.064\end{array}$

$\begin{array}{llll}0.010 & 0.251 & 0.092 & 0.091\end{array}$

$\begin{array}{lllll}0.009 & 0.233 & 0.079 & 0.111\end{array}$

$\begin{array}{llll}0.009 & 0.246 & 0.109 & 0.095\end{array}$

$\begin{array}{lllllll}0.003 & 0.093 & 0.030 & 0.037 & 0.076 & 0.136\end{array}$

$\begin{array}{lllllll}0.008 & 0.213 & 0.062 & 0.082 & 0.126 & 0.213\end{array}$ $\begin{array}{lllllll}0.007 & 0.206 & 0.054 & 0.079 & 0.122 & 0.222\end{array}$ $\begin{array}{lllllll}0.022 & 0.244 & 0.109 & 0.085 & 0.139 & 0.087\end{array}$ $\begin{array}{lllllll}0.018 & 0.251 & 0.107 & 0.070 & 0.169 & 0.092\end{array}$ $\begin{array}{lllllll}0.020 & 0.263 & 0.117 & 0.084 & 0.169 & 0.103\end{array}$ $\begin{array}{lllllll}0.020 & 0.212 & 0.106 & 0.045 & 0.174 & 0.132\end{array}$ $\begin{array}{llllllllll}0.021 & 0.222 & 0.112 & 0.050 & 0.180 & 0.135\end{array}$ $\begin{array}{lllllll}0.021 & 0.222 & 0.112 & 0.050 & 0.180 & 0.115 \\ 0.019 & 0.225 & 0.108 & 0.049 & 0.169 & 0.095\end{array}$ $\begin{array}{lllllll}0.019 & 0.225 & 0.108 & 0.049 & 0.169 & 0.095 \\ 0.031 & 0.195 & 0.103 & 0.046 & 0.334 & 0.237\end{array}$ $\begin{array}{lllllll}0.031 & 0.195 & 0.103 & 0.046 & 0.334 & 0.237\end{array}$ $\begin{array}{lllllll}0.028 & 0.205 & 0.121 & 0.033 & 0.736 & 0.511\end{array}$ $\begin{array}{lllllll}0.027 & 0.194 & 0.108 & 0.039 & 0.331 & 0.217\end{array}$ $\begin{array}{llllll}0.038 & 0.387 & 0.180 & 0.083 & 0.139 & 0.098\end{array}$ $\begin{array}{lllllll}0.040 & 0.394 & 0.188 & 0.092 & 0.224 & 0.249\end{array}$ $\begin{array}{lllllll}0.036 & 0.396 & 0.192 & 0.081 & 0.185 & 0.226\end{array}$ $\begin{array}{llllllll}0.009 & 0.216 & 0.064 & 0.081 & 0.140 & 0.190\end{array}$ $\begin{array}{lllllllll}0.009 & 0.216 & 0.064 & 0.081 & 0.140 & 0.190\end{array}$ $\begin{array}{lllllll}0.009 & 0.216 & 0.054 & 0.077 & 0.133 & 0.156 \\ 0.007 & 0.211 & 0.061 & 0.082 & 0.128 & 0.173\end{array}$ $\begin{array}{lllllll}0.007 & 0.211 & 0.061 & 0.082 & 0.128 & 0.173\end{array}$ $\begin{array}{lllllll}0.021 & 0.210 & 0.100 & 0.042 & 0.311 & 0.197\end{array}$ $\begin{array}{lllllll}0.019 & 0.216 & 0.108 & 0.049 & 0.193 & 0.141\end{array}$ $\begin{array}{lllllll}0.019 & 0.228 & 0.116 & 0.045 & 0.170 & 0.124\end{array}$ $\begin{array}{lllllll}0.012 & 0.207 & 0.076 & 0.070 & 0.137 & 0.182\end{array}$ 
Ohio Core 2626 Millbrig

1394

1395

1396

1397

1399

1400

1401

1402

1403

1404

1405

1407

1408

1409

1410

1411

1412

1413 $\begin{array}{llllll}0.012 & 0.214 & 0.076 & 0.062 & 0.142 & 0.206\end{array}$ $\begin{array}{lllllll}0.008 & 0.224 & 0.074 & 0.079 & 0.197 & 0.302\end{array}$ $\begin{array}{lllllll}0.010 & 0.212 & 0.061 & 0.079 & 0.168 & 0.294\end{array}$ $\begin{array}{lllllll}0.008 & 0.224 & 0.063 & 0.080 & 0.169 & 0.297\end{array}$ $\begin{array}{lllllll}0.011 & 0.229 & 0.077 & 0.077 & 0.126 & 0.149\end{array}$ $\begin{array}{llllll}0.008 & 0.203 & 0.068 & 0.068 & 0.113 & 0.158\end{array}$ $\begin{array}{lllllll}0.009 & 0.215 & 0.061 & 0.065 & 0.104 & 0.133\end{array}$ $\begin{array}{llllll}0.009 & 0.215 & 0.061 & 0.065 & 0.104 & 0.133 \\ 0.007 & 0.215 & 0.075 & 0.078 & 0.109 & 0.215\end{array}$ $\begin{array}{lllllll}0.007 & 0.215 & 0.075 & 0.078 & 0.109 & 0.215 \\ 0.007 & 0.216 & 0.059 & 0.085 & 0.110 & 0.207\end{array}$ $\begin{array}{lllllll}0.007 & 0.216 & 0.059 & 0.085 & 0.110 & 0.207\end{array}$ $\begin{array}{lllllll}0.011 & 0.206 & 0.068 & 0.079 & 0.143 & 0.211\end{array}$ $\begin{array}{lllllll}0.011 & 0.217 & 0.050 & 0.075 & 0.199 & 0.148\end{array}$ $\begin{array}{lllllll}0.011 & 0.210 & 0.065 & 0.078 & 0.179 & 0.143\end{array}$ $\begin{array}{lllllll}0.011 & 0.202 & 0.071 & 0.079 & 0.158 & 0.144\end{array}$ $\begin{array}{lllllll}0.007 & 0.211 & 0.069 & 0.071 & 0.121 & 0.206\end{array}$ $\begin{array}{llllll}0.008 & 0.212 & 0.055 & 0.084 & 0.125 & 0.197\end{array}$ $\begin{array}{lllllll}0.008 & 0.231 & 0.077 & 0.088 & 0.113 & 0.192\end{array}$ $\begin{array}{lllllll}0.008 & 0.231 & 0.077 & 0.088 & 0.113 & 0.192 \\ 0.009 & 0.221 & 0.106 & 0.063 & 0.067 & 0.151\end{array}$ $\begin{array}{lllllll}0.009 & 0.221 & 0.106 & 0.063 & 0.067 & 0.151 \\ 0.009 & 0.219 & 0.078 & 0.071 & 0.091 & 0.111\end{array}$ $\begin{array}{lllllll}0.009 & 0.219 & 0.078 & 0.071 & 0.091 & 0.111\end{array}$ $\begin{array}{lllllll}0.011 & 0.219 & 0.072 & 0.071 & 0.124 & 0.115\end{array}$ $\begin{array}{lllllll}0.008 & 0.200 & 0.064 & 0.080 & 0.079 & 0.086\end{array}$ $\begin{array}{lllllll}0.010 & 0.218 & 0.066 & 0.084 & 0.062 & 0.085\end{array}$ $\begin{array}{lllllll}0.007 & 0.214 & 0.053 & 0.082 & 0.068 & 0.077\end{array}$

$\begin{array}{llllll}0.041 & 0.093 & 0.030 & 0.007 & 0.402 & 0.379\end{array}$ $\begin{array}{lllllll}0.009 & 0.209 & 0.072 & 0.061 & 0.135 & 0.165\end{array}$ $\begin{array}{llllllll}0.009 & 0.209 & 0.072 & 0.061 & 0.135 & 0.165 \\ 0.008 & 0.199 & 0.075 & 0.067 & 0.086 & 0.089\end{array}$ $\begin{array}{lllllll}0.008 & 0.199 & 0.075 & 0.067 & 0.086 & 0.089 \\ 0.026 & 0.395 & 0.122 & 0.066 & 0.187 & 0.058\end{array}$ $\begin{array}{lllllll}0.026 & 0.395 & 0.122 & 0.066 & 0.187 & 0.058\end{array}$ $\begin{array}{llllll}0.026 & 0.406 & 0.136 & 0.067 & 0.132 & 0.048\end{array}$ $\begin{array}{lllllll}0.024 & 0.409 & 0.140 & 0.059 & 0.146 & 0.053\end{array}$ $\begin{array}{lllllll}0.020 & 0.164 & 0.086 & 0.051 & 0.147 & 0.083\end{array}$ $\begin{array}{lllllll}0.025 & 0.172 & 0.093 & 0.034 & 0.165 & 0.086\end{array}$ $\begin{array}{lllllll}0.024 & 0.177 & 0.102 & 0.037 & 0.138 & 0.075\end{array}$ $\begin{array}{lllllll}0.010 & 0.191 & 0.071 & 0.066 & 0.077 & 0.076\end{array}$ $\begin{array}{lllllll}0.010 & 0.182 & 0.065 & 0.062 & 0.099 & 0.079\end{array}$ $\begin{array}{lllllll}0.010 & 0.182 & 0.065 & 0.062 & 0.099 & 0.079 \\ 0.010 & 0.189 & 0.063 & 0.060 & 0.105 & 0.076\end{array}$ $\begin{array}{lllllll}0.010 & 0.189 & 0.063 & 0.060 & 0.105 & 0.076 \\ 0.009 & 0.194 & 0.072 & 0.066 & 0.107 & 0.119\end{array}$ $\begin{array}{lllllll}0.009 & 0.194 & 0.072 & 0.066 & 0.107 & 0.119 \\ 0.009 & 0.196 & 0.069 & 0.068 & 0.061 & 0.058\end{array}$ $\begin{array}{lllllll}0.009 & 0.190 & 0.057 & 0.073 & 0.059 & 0.054\end{array}$ $\begin{array}{lllllll}0.026 & 0.164 & 0.104 & 0.039 & 0.130 & 0.096\end{array}$ $\begin{array}{lllllll}0.022 & 0.155 & 0.113 & 0.031 & 0.115 & 0.092\end{array}$ $\begin{array}{llllll}0.024 & 0.156 & 0.098 & 0.037 & 0.153 & 0.086\end{array}$ $\begin{array}{lllllll}0.011 & 0.202 & 0.067 & 0.062 & 0.116 & 0.153\end{array}$ $\begin{array}{lllllll}0.011 & 0.202 & 0.067 & 0.062 & 0.116 & 0.153 \\ 0.008 & 0.195 & 0.063 & 0.055 & 0.087 & 0.091\end{array}$ $\begin{array}{lllllll}0.008 & 0.195 & 0.063 & 0.055 & 0.087 & 0.091 \\ 0.013 & 0.189 & 0.075 & 0.066 & 0.087 & 0.055\end{array}$ $\begin{array}{lllllll}0.013 & 0.189 & 0.075 & 0.066 & 0.087 & 0.055 \\ 0.008 & 0.219 & 0.069 & 0.069 & 0.152 & 0.157\end{array}$ $\begin{array}{lllllll}0.008 & 0.219 & 0.069 & 0.069 & 0.152 & 0.157\end{array}$ $\begin{array}{lllllll}0.010 & 0.191 & 0.076 & 0.062 & 0.113 & 0.093\end{array}$ $\begin{array}{llllll}0.009 & 0.193 & 0.087 & 0.066 & 0.136 & 0.103\end{array}$ $\begin{array}{lllllll}0.010 & 0.199 & 0.071 & 0.064 & 0.086 & 0.087\end{array}$ $\begin{array}{llllll}0.009 & 0.194 & 0.062 & 0.065 & 0.120 & 0.081\end{array}$ 


$$
1460
$$

Ohio Core 3256 Millbrig

$\begin{array}{lllllll}0.010 & 0.208 & 0.069 & 0.061 & 0.050 & 0.061\end{array}$ $\begin{array}{lllllll}0.010 & 0.191 & 0.069 & 0.060 & 0.078 & 0.065\end{array}$ $\begin{array}{lllllll}0.010 & 0.201 & 0.057 & 0.067 & 0.057 & 0.085\end{array}$ $\begin{array}{lllllll}0.010 & 0.201 & 0.057 & 0.067 & 0.057 & 0.085 \\ 0.042 & 0.173 & 0.145 & 0.038 & 0.166 & 0.088\end{array}$ $\begin{array}{lllllll}0.042 & 0.173 & 0.145 & 0.038 & 0.166 & 0.088 \\ 0.037 & 0.184 & 0.131 & 0.044 & 0.138 & 0.085\end{array}$ $\begin{array}{lllllll}0.037 & 0.184 & 0.131 & 0.044 & 0.138 & 0.085 \\ 0.042 & 0.172 & 0.138 & 0.041 & 0.173 & 0.090\end{array}$ $\begin{array}{lllllll}0.042 & 0.172 & 0.138 & 0.041 & 0.173 & 0.090\end{array}$ $\begin{array}{lllllll}0.010 & 0.188 & 0.072 & 0.066 & 0.095 & 0.083\end{array}$ $\begin{array}{lllllll}0.008 & 0.191 & 0.069 & 0.062 & 0.080 & 0.078\end{array}$ $\begin{array}{llllll}0.007 & 0.184 & 0.079 & 0.080 & 0.068 & 0.094\end{array}$ $\begin{array}{llllll}0.008 & 0.204 & 0.070 & 0.058 & 0.102 & 0.106\end{array}$ $\begin{array}{lllllll}0.010 & 0.188 & 0.070 & 0.072 & 0.096 & 0.089\end{array}$ $\begin{array}{lllllll}0.010 & 0.193 & 0.064 & 0.062 & 0.116 & 0.128\end{array}$ $\begin{array}{lllllll}0.010 & 0.193 & 0.064 & 0.062 & 0.116 & 0.128 \\ 0.009 & 0.215 & 0.079 & 0.057 & 0.098 & 0.137\end{array}$ $\begin{array}{lllllll}0.009 & 0.215 & 0.079 & 0.057 & 0.098 & 0.137 \\ 0.010 & 0.195 & 0.066 & 0.061 & 0.083 & 0.103\end{array}$ $\begin{array}{lllllll}0.010 & 0.195 & 0.066 & 0.061 & 0.083 & 0.103\end{array}$ $\begin{array}{lllllll}0.008 & 0.193 & 0.065 & 0.071 & 0.083 & 0.104\end{array}$ $\begin{array}{lllllll}0.007 & 0.208 & 0.073 & 0.062 & 0.138 & 0.150\end{array}$ $\begin{array}{lllllll}0.012 & 0.197 & 0.069 & 0.067 & 0.146 & 0.136\end{array}$ $\begin{array}{lllllll}0.013 & 0.197 & 0.077 & 0.063 & 0.140 & 0.142\end{array}$ $\begin{array}{lllllll}0.011 & 0.205 & 0.068 & 0.060 & 0.121 & 0.129\end{array}$ $\begin{array}{lllllll}0.007 & 0.201 & 0.076 & 0.065 & 0.103 & 0.093\end{array}$ $\begin{array}{llllllll}0.011 & 0.214 & 0.070 & 0.068 & 0.112 & 0.122\end{array}$ $\begin{array}{lllllll}0.011 & 0.214 & 0.070 & 0.068 & 0.112 & 0.122 \\ 0.010 & 0.198 & 0.102 & 0.061 & 0.103 & 0.094\end{array}$ $\begin{array}{lllllll}0.010 & 0.198 & 0.102 & 0.061 & 0.103 & 0.094 \\ 0.008 & 0.218 & 0.081 & 0.065 & 0.114 & 0.098\end{array}$ $\begin{array}{lllllll}0.008 & 0.218 & 0.081 & 0.065 & 0.114 & 0.098\end{array}$ $\begin{array}{llllll}0.011 & 0.216 & 0.076 & 0.059 & 0.138 & 0.112\end{array}$ $\begin{array}{lllllll}0.018 & 0.193 & 0.120 & 0.056 & 0.078 & 0.067\end{array}$ $\begin{array}{lllllll}0.017 & 0.197 & 0.106 & 0.041 & 0.069 & 0.052\end{array}$ $\begin{array}{llllll}0.017 & 0.209 & 0.099 & 0.052 & 0.105 & 0.093\end{array}$ $\begin{array}{lllllll}0.010 & 0.205 & 0.067 & 0.063 & 0.075 & 0.088\end{array}$ $\begin{array}{lllllll}0.011 & 0.202 & 0.084 & 0.072 & 0.090 & 0.105\end{array}$ $\begin{array}{llllll}0.011 & 0.202 & 0.084 & 0.072 & 0.090 & 0.105\end{array}$ $\begin{array}{lllllll}0.009 & 0.195 & 0.057 & 0.068 & 0.085 & 0.112 \\ 0.010 & 0.185 & 0.068 & 0.067 & 0.139 & 0.114\end{array}$ $\begin{array}{lllllll}0.010 & 0.185 & 0.068 & 0.067 & 0.139 & 0.114 \\ 0.009 & 0.206 & 0.063 & 0.064 & 0.159 & 0.110\end{array}$ $\begin{array}{llllll}0.009 & 0.206 & 0.063 & 0.064 & 0.159 & 0.110\end{array}$ $\begin{array}{lllllll}0.009 & 0.206 & 0.059 & 0.071 & 0.148 & 0.108\end{array}$ $\begin{array}{lllllll}0.010 & 0.206 & 0.068 & 0.065 & 0.105 & 0.088\end{array}$ $\begin{array}{lllllll}0.010 & 0.210 & 0.072 & 0.077 & 0.079 & 0.076\end{array}$ $\begin{array}{lllllll}0.010 & 0.202 & 0.065 & 0.066 & 0.090 & 0.103\end{array}$

$\begin{array}{llllll}0.011 & 0.198 & 0.067 & 0.065 & 0.092 & 0.079\end{array}$ $\begin{array}{lllllll}0.011 & 0.198 & 0.067 & 0.065 & 0.092 & 0.079\end{array}$ $\begin{array}{lllllll}0.011 & 0.217 & 0.069 & 0.065 & 0.098 & 0.079 \\ 0.009 & 0.200 & 0.071 & 0.067 & 0.099 & 0.080\end{array}$ $\begin{array}{lllllll}0.009 & 0.200 & 0.071 & 0.067 & 0.099 & 0.080 \\ 0.012 & 0.196 & 0.066 & 0.074 & 0.129 & 0.116\end{array}$ $\begin{array}{lllllll}0.012 & 0.196 & 0.066 & 0.074 & 0.129 & 0.116\end{array}$ $\begin{array}{lllllll}0.009 & 0.197 & 0.078 & 0.068 & 0.096 & 0.094\end{array}$ $\begin{array}{lllllll}0.007 & 0.203 & 0.073 & 0.061 & 0.105 & 0.088\end{array}$ $\begin{array}{lllllll}0.008 & 0.198 & 0.060 & 0.074 & 0.079 & 0.099\end{array}$ $\begin{array}{lllllll}0.008 & 0.187 & 0.060 & 0.077 & 0.137 & 0.106\end{array}$ 

$\begin{array}{lllllll}0.008 & 0.199 & 0.000 & 0.070 & 0.000 & 0.107\end{array}$ $\begin{array}{lllllll}0.008 & 0.200 & 0.000 & 0.071 & 0.002 & 0.096\end{array}$ $\begin{array}{llllll}0.009 & 0.226 & 0.000 & 0.065 & 0.002 & 0.135\end{array}$ $\begin{array}{lllllll}0.007 & 0.204 & 0.001 & 0.069 & 0.003 & 0.109\end{array}$ $\begin{array}{lllllll}0.006 & 0.222 & 0.000 & 0.069 & 0.001 & 0.103\end{array}$ $\begin{array}{lllllll}0.006 & 0.222 & 0.000 & 0.069 & 0.001 & 0.103 \\ 0.007 & 0.215 & 0.000 & 0.073 & 0.002 & 0.111\end{array}$ $\begin{array}{llllll}0.007 & 0.215 & 0.000 & 0.073 & 0.002 & 0.111 \\ 0.008 & 0.208 & 0.000 & 0.070 & 0.002 & 0.060\end{array}$ $\begin{array}{llllll}0.008 & 0.208 & 0.000 & 0.070 & 0.002 & 0.060 \\ 0.008 & 0.218 & 0.000 & 0.069 & 0.001 & 0.082\end{array}$ $\begin{array}{lllllll}0.008 & 0.218 & 0.000 & 0.069 & 0.001 & 0.082 \\ 0.009 & 0.199 & 0.000 & 0.075 & 0.000 & 0.099\end{array}$ $\begin{array}{lllllll}0.007 & 0.199 & 0.000 & 0.078 & 0.001 & 0.078\end{array}$ $\begin{array}{lllllll}0.009 & 0.201 & 0.000 & 0.065 & 0.000 & 0.090\end{array}$ $\begin{array}{llllll}0.011 & 0.202 & 0.064 & 0.081 & 0.122 & 0.108\end{array}$ $\begin{array}{lllllll}0.012 & 0.201 & 0.067 & 0.064 & 0.109 & 0.099\end{array}$ $\begin{array}{lllllll}0.010 & 0.218 & 0.069 & 0.070 & 0.176 & 0.219\end{array}$ $\begin{array}{lllllll}0.010 & 0.218 & 0.069 & 0.070 & 0.176 & 0.219\end{array}$ $\begin{array}{llllllll}0.011 & 0.214 & 0.076 & 0.066 & 0.124 & 0.139\end{array}$ $\begin{array}{lllllll}0.010 & 0.213 & 0.058 & 0.059 & 0.109 & 0.130\end{array}$ $\begin{array}{lllllll}0.010 & 0.214 & 0.068 & 0.062 & 0.130 & 0.143\end{array}$ $\begin{array}{lllllll}0.010 & 0.187 & 0.064 & 0.062 & 0.096 & 0.091\end{array}$ $\begin{array}{lllllll}0.010 & 0.181 & 0.070 & 0.073 & 0.108 & 0.086\end{array}$ $\begin{array}{lllllll}0.008 & 0.202 & 0.076 & 0.066 & 0.116 & 0.099\end{array}$ $\begin{array}{lllllll}0.009 & 0.215 & 0.068 & 0.063 & 0.096 & 0.117\end{array}$ $\begin{array}{lllllll}0.010 & 0.207 & 0.063 & 0.070 & 0.082 & 0.100\end{array}$ $\begin{array}{llllllll}0.007 & 0.209 & 0.062 & 0.061 & 0.095 & 0.111\end{array}$ $\begin{array}{lllllll}0.0010 & 0.209 & 0.062 & 0.061 & 0.095 & 0.111\end{array}$ $\begin{array}{lllllll}0.010 & 0.204 & 0.052 & 0.071 & 0.147 & 0.166\end{array}$ $\begin{array}{lllllll}0.008 & 0.205 & 0.060 & 0.081 & 0.155 & 0.167\end{array}$ $\begin{array}{lllllll}0.010 & 0.200 & 0.072 & 0.079 & 0.133 & 0.148\end{array}$ $\begin{array}{lllllll}0.011 & 0.202 & 0.083 & 0.064 & 0.177 & 0.160\end{array}$ $\begin{array}{lllllll}0.011 & 0.199 & 0.062 & 0.065 & 0.138 & 0.125\end{array}$ $\begin{array}{lllllll}0.011 & 0.196 & 0.068 & 0.072 & 0.101 & 0.118\end{array}$ $\begin{array}{lllllll}0.012 & 0.203 & 0.071 & 0.066 & 0.105 & 0.099\end{array}$ $\begin{array}{lllllll}0.010 & 0.197 & 0.067 & 0.077 & 0.080 & 0.089\end{array}$ $\begin{array}{llllllll}0.010 & 0.197 & 0.069 & 0.066 & 0.097 & 0.098\end{array}$ $\begin{array}{llllllll}0.010 & 0.197 & 0.069 & 0.066 & 0.097 & 0.098\end{array}$ $\begin{array}{lllllll}0.011 & 0.191 & 0.113 & 0.067 & 0.083 & 0.096 \\ 0.009 & 0.200 & 0.071 & 0.066 & 0.091 & 0.083\end{array}$ $\begin{array}{lllllll}0.009 & 0.200 & 0.071 & 0.066 & 0.091 & 0.083\end{array}$ $\begin{array}{llllll}0.009 & 0.206 & 0.072 & 0.063 & 0.096 & 0.095\end{array}$ $\begin{array}{llllll}0.009 & 0.228 & 0.055 & 0.063 & 0.102 & 0.108\end{array}$ $\begin{array}{llllll}0.010 & 0.218 & 0.080 & 0.073 & 0.064 & 0.100\end{array}$ $\begin{array}{llllll}0.010 & 0.223 & 0.072 & 0.066 & 0.095 & 0.112\end{array}$ Shakertown, Kentucky Millbrig

$\begin{array}{lllllll}0.053 & 0.384 & 0.206 & 0.061 & 0.228 & 0.226\end{array}$ $\begin{array}{llllllll}0.053 & 0.384 & 0.206 & 0.061 & 0.228 & 0.226\end{array}$ $\begin{array}{llllllll}0.051 & 0.372 & 0.186 & 0.062 & 0.229 & 0.225 \\ 0.052 & 0.381 & 0.198 & 0.059 & 0.164 & 0.167\end{array}$ $\begin{array}{lllllll}0.052 & 0.381 & 0.198 & 0.059 & 0.164 & 0.167\end{array}$ $\begin{array}{lllllll}0.010 & 0.257 & 0.077 & 0.083 & 0.095 & 0.101\end{array}$ $\begin{array}{lllllll}0.010 & 0.240 & 0.073 & 0.084 & 0.097 & 0.116\end{array}$ $\begin{array}{lllllll}0.010 & 0.242 & 0.077 & 0.073 & 0.092 & 0.116\end{array}$ $\begin{array}{lllllll}0.049 & 0.378 & 0.211 & 0.074 & 0.168 & 0.157\end{array}$ $\begin{array}{lllllll}0.048 & 0.389 & 0.168 & 0.071 & 0.184 & 0.128\end{array}$ 
$\begin{array}{llllll}0.051 & 0.358 & 0.194 & 0.071 & 0.161 & 0.152\end{array}$ $\begin{array}{lllllll}0.011 & 0.254 & 0.076 & 0.076 & 0.116 & 0.111\end{array}$ $\begin{array}{lllllll}0.010 & 0.260 & 0.092 & 0.077 & 0.106 & 0.138\end{array}$ $\begin{array}{lllllll}0.009 & 0.248 & 0.071 & 0.072 & 0.109 & 0.102\end{array}$ $\begin{array}{llllll}0.009 & 0.244 & 0.069 & 0.085 & 0.121 & 0.130\end{array}$ $\begin{array}{llllll}0.008 & 0.221 & 0.054 & 0.083 & 0.158 & 0.140\end{array}$ $\begin{array}{lllllll}0.011 & 0.253 & 0.068 & 0.091 & 0.100 & 0.117\end{array}$ $\begin{array}{llllll}0.011 & 0.253 & 0.068 & 0.091 & 0.100 & 0.117 \\ 0.045 & 0.387 & 0.248 & 0.089 & 0.107 & 0.146\end{array}$ $\begin{array}{lllllll}0.045 & 0.387 & 0.248 & 0.089 & 0.107 & 0.146 \\ 0.044 & 0.385 & 0.260 & 0.088 & 0.094 & 0.167\end{array}$ $\begin{array}{lllllll}0.044 & 0.385 & 0.260 & 0.088 & 0.094 & 0.167\end{array}$ $\begin{array}{lllllll}0.043 & 0.385 & 0.231 & 0.089 & 0.116 & 0.164\end{array}$ $\begin{array}{lllllll}0.054 & 0.367 & 0.188 & 0.066 & 0.117 & 0.091\end{array}$ $\begin{array}{lllllll}0.053 & 0.396 & 0.187 & 0.067 & 0.133 & 0.078\end{array}$ $\begin{array}{lllllll}0.051 & 0.374 & 0.198 & 0.062 & 0.115 & 0.089\end{array}$ $\begin{array}{lllllll}0.050 & 0.380 & 0.179 & 0.067 & 0.169 & 0.134\end{array}$ $\begin{array}{llllll}0.052 & 0.366 & 0.212 & 0.067 & 0.151 & 0.161\end{array}$ $\begin{array}{lllllll}0.051 & 0.390 & 0.191 & 0.061 & 0.150 & 0.140\end{array}$ $\begin{array}{llllll}0.051 & 0.390 & 0.191 & 0.061 & 0.150 & 0.140 \\ 0.009 & 0.244 & 0.076 & 0.081 & 0.080 & 0.128\end{array}$ $\begin{array}{llllll}0.009 & 0.244 & 0.076 & 0.081 & 0.080 & 0.128 \\ 0.007 & 0.232 & 0.061 & 0.089 & 0.061 & 0.057\end{array}$ $\begin{array}{lllllll}0.007 & 0.232 & 0.061 & 0.089 & 0.061 & 0.057\end{array}$ $\begin{array}{lllllll}0.009 & 0.235 & 0.076 & 0.083 & 0.057 & 0.106\end{array}$ $\begin{array}{lllllll}0.005 & 0.246 & 0.052 & 0.094 & 0.093 & 0.125\end{array}$ $\begin{array}{llllll}0.009 & 0.247 & 0.082 & 0.086 & 0.093 & 0.146\end{array}$ $\begin{array}{lllllll}0.008 & 0.258 & 0.064 & 0.081 & 0.104 & 0.137\end{array}$ $\begin{array}{lllllll}0.011 & 0.243 & 0.092 & 0.072 & 0.109 & 0.102\end{array}$ $\begin{array}{lllllll}0.010 & 0.240 & 0.068 & 0.076 & 0.103 & 0.092\end{array}$ $\begin{array}{llllllll}0.010 & 0.264 & 0.078 & 0.076 & 0.076 & 0.103\end{array}$ $\begin{array}{lllllll}0.010 & 0.264 & 0.078 & 0.076 & 0.076 & 0.103\end{array}$ $\begin{array}{lllllll}0.050 & 0.332 & 0.189 & 0.060 & 0.120 & 0.101 \\ 0.053 & 0.319 & 0.200 & 0.066 & 0.122 & 0.104\end{array}$ $\begin{array}{lllllll}0.053 & 0.319 & 0.200 & 0.066 & 0.122 & 0.104\end{array}$ $\begin{array}{lllllll}0.049 & 0.337 & 0.165 & 0.072 & 0.133 & 0.094\end{array}$ $\begin{array}{lllllll}0.010 & 0.232 & 0.076 & 0.087 & 0.098 & 0.219\end{array}$ $\begin{array}{lllllll}0.006 & 0.250 & 0.052 & 0.086 & 0.168 & 0.234\end{array}$ $\begin{array}{llllll}0.007 & 0.239 & 0.075 & 0.089 & 0.103 & 0.163\end{array}$ $\begin{array}{llllll}0.009 & 0.251 & 0.078 & 0.075 & 0.193 & 0.104\end{array}$ $\begin{array}{lllllll}0.012 & 0.226 & 0.069 & 0.075 & 0.235 & 0.190\end{array}$ $\begin{array}{lllllll}0.012 & 0.226 & 0.069 & 0.075 & 0.235 & 0.190 \\ 0.009 & 0.251 & 0.086 & 0.071 & 0.299 & 0.209\end{array}$ $\begin{array}{lllllll}0.009 & 0.251 & 0.086 & 0.071 & 0.299 & 0.209 \\ 0.010 & 0.234 & 0.092 & 0.078 & 0.085 & 0.101\end{array}$ $\begin{array}{lllllll}0.010 & 0.234 & 0.092 & 0.078 & 0.085 & 0.101 \\ 0.012 & 0.261 & 0.062 & 0.076 & 0.112 & 0.103\end{array}$ $\begin{array}{llllll}0.012 & 0.261 & 0.062 & 0.076 & 0.112 & 0.103\end{array}$ $\begin{array}{lllllll}0.009 & 0.235 & 0.082 & 0.078 & 0.103 & 0.117\end{array}$ $\begin{array}{llllll}0.011 & 0.258 & 0.071 & 0.075 & 0.143 & 0.117\end{array}$ $\begin{array}{llllll}0.009 & 0.252 & 0.085 & 0.074 & 0.120 & 0.113\end{array}$ $\begin{array}{lllllll}0.012 & 0.243 & 0.067 & 0.076 & 0.133 & 0.115\end{array}$ $\begin{array}{lllllll}0.010 & 0.249 & 0.071 & 0.079 & 0.111 & 0.120\end{array}$ $\begin{array}{lllllll}0.010 & 0.231 & 0.070 & 0.077 & 0.066 & 0.063\end{array}$ $\begin{array}{lllllll}0.010 & 0.231 & 0.070 & 0.077 & 0.066 & 0.063 \\ 0.008 & 0.243 & 0.075 & 0.075 & 0.077 & 0.085\end{array}$ $\begin{array}{lllllll}0.008 & 0.243 & 0.075 & 0.075 & 0.077 & 0.085 \\ 0.009 & 0.141 & 0.084 & 0.075 & 0.101 & 0.101\end{array}$ $\begin{array}{lllllll}0.009 & 0.141 & 0.084 & 0.075 & 0.101 & 0.101 \\ 0.010 & 0.139 & 0.099 & 0.083 & 0.092 & 0.133\end{array}$ $\begin{array}{lllllll}0.010 & 0.139 & 0.099 & 0.083 & 0.092 & 0.133\end{array}$ $\begin{array}{lllllll}0.015 & 0.154 & 0.076 & 0.082 & 0.105 & 0.087\end{array}$ $\begin{array}{lllllll}0.020 & 0.318 & 0.138 & 0.073 & 0.168 & 0.114\end{array}$ $\begin{array}{lllllll}0.019 & 0.314 & 0.119 & 0.060 & 0.196 & 0.105\end{array}$ $\begin{array}{lllllll}0.018 & 0.299 & 0.123 & 0.074 & 0.188 & 0.111\end{array}$ 
Dickeyville, Wisconsin Millbrig

1571

1572

1573

1574

1575

1576
1577

1578

1578

1579

1581

1582

1583

1584

1585

1586

1587

1588

1590

1591

1592

1593

1594

1596

1596

1597

1599

1600

1601

1602

1603

1604

1605

1606

1607

1609

1610

1611

$\begin{array}{llllll}0.011 & 0.245 & 0.091 & 0.073 & 0.094 & 0.089\end{array}$ $\begin{array}{llllll}0.010 & 0.261 & 0.073 & 0.089 & 0.095 & 0.103\end{array}$ $\begin{array}{llllll}0.010 & 0.258 & 0.092 & 0.082 & 0.118 & 0.128\end{array}$ $\begin{array}{llllll}0.011 & 0.252 & 0.085 & 0.082 & 0.099 & 0.112\end{array}$ $\begin{array}{lllllll}0.011 & 0.243 & 0.070 & 0.076 & 0.097 & 0.109\end{array}$ $\begin{array}{lllllll}0.011 & 0.248 & 0.083 & 0.085 & 0.095 & 0.111\end{array}$ $\begin{array}{lllllll}0.011 & 0.238 & 0.078 & 0.077 & 0.074 & 0.092\end{array}$ $\begin{array}{lllllll}0.053 & 0.156 & 0.603 & 0.061 & 0.069 & 0.060\end{array}$ $\begin{array}{lllllll}0.012 & 0.259 & 0.075 & 0.069 & 0.088 & 0.094\end{array}$ $\begin{array}{lllllll}0.012 & 0.259 & 0.075 & 0.069 & 0.088 & 0.094\end{array}$ $\begin{array}{lllllll}0.009 & 0.229 & 0.127 & 0.087 & 0.108 & 0.107\end{array}$ $\begin{array}{lllllll}0.009 & 0.241 & 0.068 & 0.073 & 0.128 & 0.097\end{array}$ $\begin{array}{lllllll}0.011 & 0.222 & 0.071 & 0.082 & 0.097 & 0.084\end{array}$ $\begin{array}{llllll}0.011 & 0.235 & 0.081 & 0.072 & 0.097 & 0.088\end{array}$ $\begin{array}{lllllll}0.011 & 0.243 & 0.111 & 0.076 & 0.082 & 0.086\end{array}$ $\begin{array}{lllllll}0.009 & 0.253 & 0.061 & 0.079 & 0.101 & 0.094\end{array}$ $\begin{array}{lllllll}0.009 & 0.257 & 0.093 & 0.076 & 0.105 & 0.117\end{array}$ $\begin{array}{lllllll}0.010 & 0.264 & 0.082 & 0.084 & 0.000 & 0.110\end{array}$ $\begin{array}{llllllll}0.012 & 0.269 & 0.081 & 0.082 & 0.108 & 0.109\end{array}$ $\begin{array}{lllllll}0.012 & 0.269 & 0.081 & 0.082 & 0.108 & 0.109 \\ 0.010 & 0.265 & 0.077 & 0.075 & 0.000 & 0.220\end{array}$ $\begin{array}{lllllll}0.010 & 0.265 & 0.077 & 0.075 & 0.000 & 0.220 \\ 0.009 & 0.257 & 0.083 & 0.080 & 0.109 & 0.138\end{array}$ $\begin{array}{lllllll}0.009 & 0.257 & 0.083 & 0.080 & 0.109 & 0.138 \\ 0.011 & 0.266 & 0.075 & 0.078 & 0.165 & 0.150\end{array}$ $\begin{array}{lllllll}0.010 & 0.250 & 0.069 & 0.095 & 0.125 & 0.163\end{array}$ $\begin{array}{llllll}0.009 & 0.247 & 0.074 & 0.091 & 0.114 & 0.135\end{array}$ $\begin{array}{lllllll}0.007 & 0.241 & 0.084 & 0.100 & 0.086 & 0.149\end{array}$ $\begin{array}{lllllll}0.027 & 0.228 & 0.112 & 0.037 & 0.267 & 0.152\end{array}$ $\begin{array}{lllllll}0.030 & 0.222 & 0.117 & 0.037 & 0.149 & 0.152\end{array}$ $\begin{array}{llllllll}0.028 & 0.222 & 0.123 & 0.040 & 0.177 & 0.113\end{array}$ $\begin{array}{lllllll}0.028 & 0.222 & 0.123 & 0.040 & 0.177 & 0.113\end{array}$ $\begin{array}{llllllll}0.009 & 0.257 & 0.087 & 0.082 & 0.126 & 0.122 \\ 0.009 & 0.255 & 0.075 & 0.069 & 0.124 & 0.120\end{array}$ $\begin{array}{lllllll}0.009 & 0.255 & 0.075 & 0.069 & 0.124 & 0.120\end{array}$ $\begin{array}{lllllll}0.011 & 0.255 & 0.078 & 0.077 & 0.113 & 0.125\end{array}$ $\begin{array}{lllllll}0.011 & 0.261 & 0.085 & 0.085 & 0.000 & 0.127\end{array}$ $\begin{array}{lllllll}0.013 & 0.236 & 0.103 & 0.084 & 0.109 & 0.119\end{array}$ $\begin{array}{lllllll}0.011 & 0.256 & 0.076 & 0.074 & 0.114 & 0.136\end{array}$ $\begin{array}{lllllll}0.044 & 0.229 & 0.115 & 0.033 & 0.100 & 0.064\end{array}$ $\begin{array}{lllllll}0.043 & 0.219 & 0.1102 & 0.029 & 0.105 & 0.058\end{array}$ $\begin{array}{llllllll}0.043 & 0.219 & 0.102 & 0.029 & 0.105 & 0.058\end{array}$ $\begin{array}{llllllll}0.039 & 0.221 & 0.104 & 0.030 & 0.082 & 0.058 \\ 0.010 & 0.260 & 0.070 & 0.077 & 0.090 & 0.116\end{array}$ $\begin{array}{lllllll}0.010 & 0.260 & 0.070 & 0.077 & 0.090 & 0.116 \\ 0.006 & 0.237 & 0.061 & 0.081 & 0.109 & 0.120\end{array}$ $\begin{array}{lllllll}0.006 & 0.237 & 0.061 & 0.081 & 0.109 & 0.120\end{array}$ $\begin{array}{lllllll}0.010 & 0.226 & 0.053 & 0.102 & 0.297 & 0.132\end{array}$ $\begin{array}{lllllll}0.011 & 0.246 & 0.073 & 0.081 & 0.104 & 0.127\end{array}$ $\begin{array}{lllllll}0.011 & 0.260 & 0.080 & 0.076 & 0.098 & 0.121\end{array}$ $\begin{array}{llllll}0.014 & 0.249 & 0.092 & 0.083 & 0.108 & 0.104\end{array}$ 
$\begin{array}{llllll}0.012 & 0.272 & 0.072 & 0.077 & 0.125 & 0.130\end{array}$

$\begin{array}{lllllll}0.009 & 0.253 & 0.083 & 0.086 & 0.099 & 0.107\end{array}$

$\begin{array}{lllllll}0.011 & 0.252 & 0.066 & 0.073 & 0.170 & 0.190\end{array}$

$\begin{array}{llllll}0.010 & 0.249 & 0.084 & 0.079 & 0.113 & 0.119\end{array}$

$\begin{array}{lllllll}0.013 & 0.256 & 0.066 & 0.065 & 0.091 & 0.110\end{array}$

$\begin{array}{lllllll}0.012 & 0.234 & 0.070 & 0.075 & 0.103 & 0.103\end{array}$

$\begin{array}{lllllll}0.012 & 0.248 & 0.119 & 0.075 & 0.126 & 0.084\end{array}$

$\begin{array}{lllllll}0.012 & 0.248 & 0.119 & 0.075 & 0.126 & 0.084 \\ 0.014 & 0.254 & 0.084 & 0.081 & 0.102 & 0.079\end{array}$

$\begin{array}{lllllll}0.012 & 0.247 & 0.146 & 0.078 & 0.147 & 0.098\end{array}$

1619
1620 

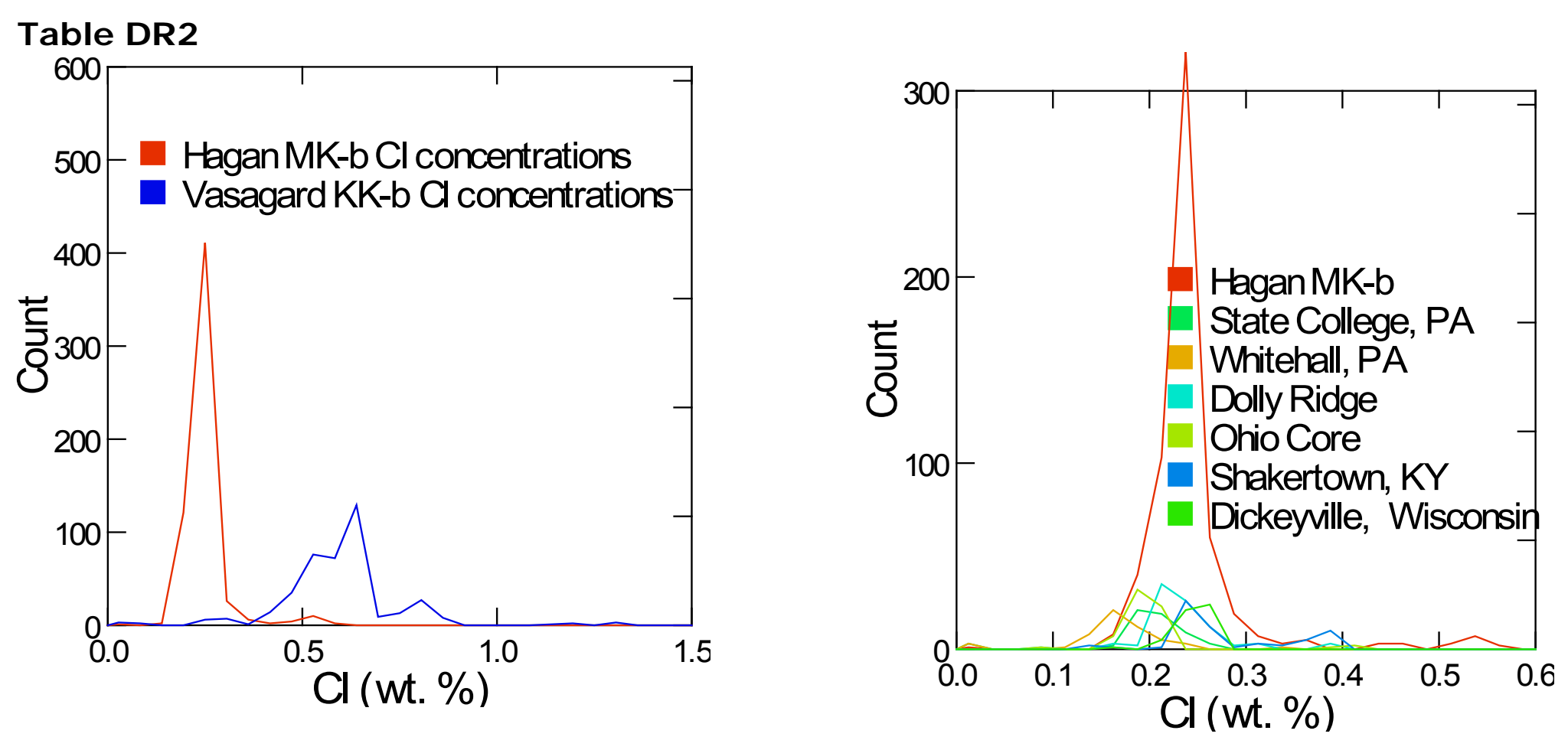

Comparison of $\mathrm{Cl}$ analyses from Hagan MKK-b and Vasagard KrComparison of $\mathrm{Cl}$ analyses from MK-b samples

\begin{tabular}{|c|c|c|c|c|c|c|c|c|c|c|}
\hline & MK-b & KK-b & & Hagan & State $\mathrm{C}$ & Whiteh & Dolly R & Ohio & Kentuc & Wisconsin \\
\hline $\mathrm{N}$ of Cases & 585 & 408 & $\mathrm{~N}$ of Cases & 585 & 57 & 54 & 87 & 66 & 63 & 51 \\
\hline Range & 0.564 & 1.328 & Range & 0.564 & 0.256 & 0.332 & 0.303 & 0.316 & 0.257 & 0.116 \\
\hline Median & 0.233 & 0.6075 & Median & 0.233 & 0.202 & 0.166 & 0.225 & 0.196 & 0.251 & 0.249 \\
\hline Arithmetic Mean & 0.2418 & 0.5961 & Arithmetic Mean & 0.242 & 0.198 & 0.167 & 0.234 & 0.202 & 0.279 & 0.245 \\
\hline Standard Deviation & 0.0558 & 0.1506 & Standard Deviation & 0.056 & 0.051 & 0.053 & 0.043 & 0.048 & 0.066 & 0.019 \\
\hline Variance & 0.0031 & 0.0227 & Variance & 0.003 & 0.003 & 0.003 & 0.002 & 0.002 & 0.004 & $3 E-04$ \\
\hline Shapiro-Wilk Statistic & 0.5949 & 0.866 & Shapiro-Wilk Statistic & ( 0.595 & 0.65 & 0.84 & 0.831 & 0.541 & 0.854 & 0.825 \\
\hline
\end{tabular}

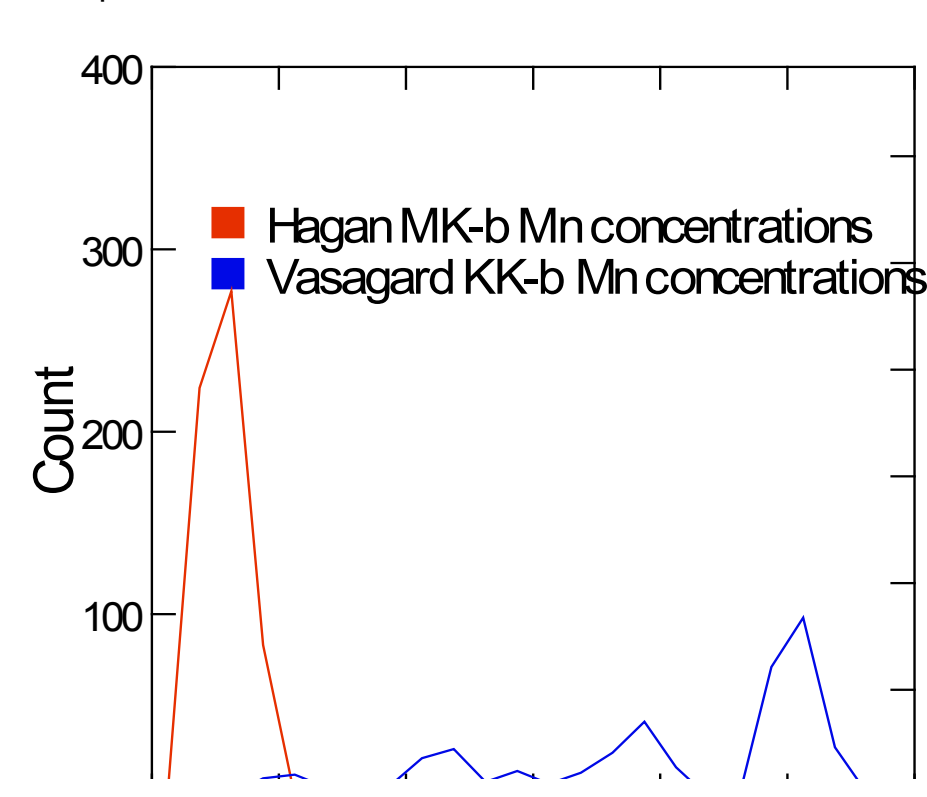

Note: Whitehall, PA samples have been subjected to the most extreme metamorphic conditions out of the entire group.

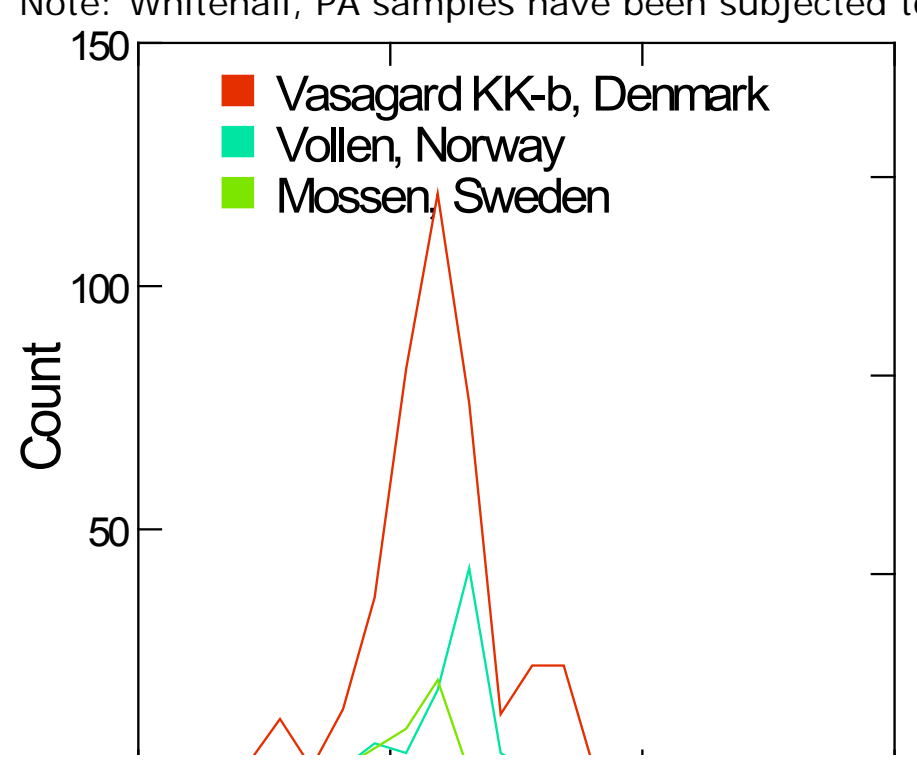



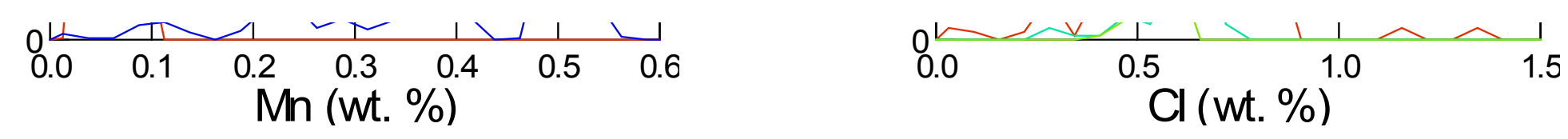

Comparison of $\mathrm{Mn}$ analyses from Hagan MKK-b and Vasagard KComparison of $\mathrm{Cl}$ analyses for KK-b samples

\begin{tabular}{lrrrrrrr} 
& MK-b & KK-b & & & \multicolumn{2}{c}{ Denma Norwaj Sweden } \\
N of Cases & 585 & 408 & N of Cases & 408 & 78 & 34 \\
Range & 0.564 & 1.328 & Range & 1.328 & 0.437 & 0.22 \\
Median & 0.233 & 0.6075 & Median & 0.608 & 0.635 & 0.575 \\
Arithmetic Mean & 0.2418 & 0.5961 & Arithmetic Mean & 0.596 & 0.602 & 0.557 \\
Standard Deviation & 0.0558 & 0.1506 & & Standard Deviation & 0.151 & 0.092 & 0.054 \\
Variance & 0.0031 & 0.0227 & & Variance & 0.023 & 0.008 & 0.003 \\
Shapiro-Wilk Statistic & 0.5949 & 0.866 & & Shapiro-Wilk Statistic & 0.866 & 0.786 & 0.864 \\
& & & & & Note: all three samples come from different
\end{tabular}

Shapiro-Wilk Statistic $\quad 0.5949 \quad 0.866$

Note: all three samples come from different sedimentary environments and were subjected to very different metamorphic conditions.

Hagan Mg

Bin

Hagan $\mathrm{C}$

$$
\mathrm{Cl} \text { Bin Value Bin Bin Value }
$$

$\begin{array}{rrrrlr}1.25 \mathrm{E}-03 & 1 & 0.1525 & 0 & 0.051 & \\ 3.75 \mathrm{E}-03 & 0 & 0.1575 & 1 & 0.054 & 6 \\ 6.25 \mathrm{E}-03 & 16 & 0.1625 & 1 & 0.056 & 14 \\ 8.75 \mathrm{E}-03 & 91 & 0.1675 & 0 & 0.059 & 12 \\ 0.01125 & 94 & 0.1725 & 6 & 0.061 & 8 \\ 0.01375 & 14 & 0.1775 & 12 & 0.064 & 20 \\ 0.01625 & 50 & 0.1825 & 6 & 0.066 & 24 \\ 0.01875 & 90 & 0.1875 & 11 & 0.069 & 14 \\ 0.02125 & 55 & 0.1925 & 5 & 0.071 & 24 \\ 0.02375 & 19 & 0.1975 & 6 & 0.074 & 17 \\ 0.02625 & 30 & 0.2025 & 13 & 0.076 & 22 \\ 0.02875 & 12 & 0.2075 & 14 & 0.079 & 10 \\ 0.03125 & 20 & 0.2125 & 12 & 0.081 & 13 \\ 0.03375 & 7 & 0.2175 & 21 & 0.084 & 8 \\ 0.03625 & 3 & 0.2225 & 43 & 0.086 & 11 \\ 0.03875 & 1 & 0.2275 & 70 & 0.089 & 3 \\ 0.04125 & 8 & 0.2325 & 98 & 0.091 & 6 \\ 0.04375 & 5 & 0.2375 & 67 & 0.094 & 4 \\ 0.04625 & 12 & 0.2425 & 55 & 0.096 & 6 \\ 0.04875 & 3 & 0.2475 & 31 & 0.099 & 17 \\ 0.05125 & 9 & 0.2525 & 26 & 0.101 & 22 \\ 0.05375 & 1 & 0.2575 & 14 & 0.104 & 24 \\ 0.05625 & 0 & 0.2625 & 6 & 0.106 & 21 \\ 0.05875 & 0 & 0.2675 & 8 & 0.109 & 24 \\ 0.06125 & 0 & 0.2725 & 6 & 0.111 & 18 \\ 0.06375 & 1 & 0.2775 & 3 & 0.114 & 14 \\ 0.06625 & 4 & 0.2825 & 9 & 0.116 & 32 \\ 0.06875 & 6 & 0.2875 & 2 & 0.119 & 3 \\ 0.07125 & 2 & 0.2925 & 0.121 & \\ 0.07375 & 3 & 0.2975 & 2 & 0.124 & \end{array}$

Hagan $\mathrm{Mn}$

2


$\begin{array}{lll}0.07625 & 14 & 0.3025 \\ 0.07875 & & 0.3075\end{array}$

$\begin{array}{lll}0.07875 & 3 & 0.3075 \\ 0.08125 & 6 & 0.3125\end{array}$

$\begin{array}{lll}0.08125 & 6 & 0.3125\end{array}$

$0.08375 \quad 10.3175$

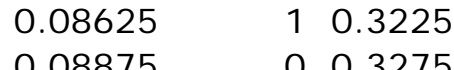

$\begin{array}{ll}3 & 0.126 \\ 0 & 0.129\end{array}$

$\begin{array}{ll}0 & 0.129 \\ 0 & 0.131\end{array}$

$\begin{array}{ll}0 & 0.131 \\ 2 & 0.134\end{array}$

$\begin{array}{ll}2 & 0.134 \\ 2 & 0.136\end{array}$

0.08875

$\begin{array}{ll}2 & 0.139 \\ 0 & 0.141\end{array}$

$\begin{array}{lll}0.08875 & 0 & 0.3275 \\ 0.09125 & 0 & 0.3325 \\ 0.09375 & 0 & 0.3375\end{array}$

0.09625

0.09875

$\begin{array}{ll}0 & 0.146 \\ 0 & 0.149\end{array}$

$\begin{array}{ll}0 & 0.149\end{array}$

14
9
10
5
10
6
6
4
5
4




\section{Table DR3}

Each sub-sample is treated as a separate entity. It is assumed that each analysis is a random sample of the total population of apatite crystals.

The F-value is used to compare variation among the sample means of each sub-sample with the their variation.

If the $\mathrm{F}$-value is less than or close to 1.00 , then the mean values of the samples compared are likely the same and the null hypothesis cannot be rejected.

If the $p$-value is less than or equal to 0.000 , then the probability is very low that the compared samples are the same.

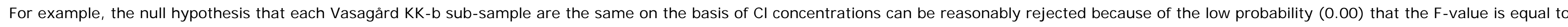

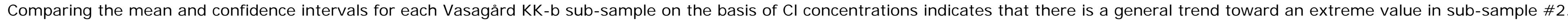

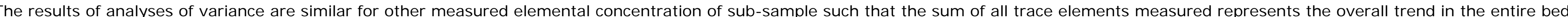

The use of ANOVA statistics for comparing apatite samples should be regarded with skepticism because the apatite trace element concentrations are not always normally distributed.

However, the approach appears reasonable because the statistical interpretation does not change the general conclusions made on the basis visual comparison of data plots.

The Vasagård KK-b null hypothesis is that all sub-samples cannot be distinguished on the basis of apatite chemistry, i.e. all sub-samples are similar.

Analyzing the variance of all trace elements for the Vasagård KK-b sub-samples yields a very high F-value with a very low probability.

The Vasagård K-b null hypothesis can be rejected.

\begin{tabular}{|c|c|c|c|c|}
\hline \multicolumn{5}{|c|}{$11+2)^{\prime}$} \\
\hline VasKK-b1 & 45 & 1.9802 & 0.3916 & 0.09 \\
\hline VasKK-b2 & 57 & 1.7521 & 0.3603 & 0.08 \\
\hline VasKK-b3 & 60 & 2.1973 & 0.3427 & 0.08 \\
\hline VasKK-b4 & 63 & 2.2678 & 0.1828 & 0.07 \\
\hline VasKK-b5 & 60 & 2.2142 & 0.4441 & 0.08 \\
\hline VaskK-b6 & 60 & 2.1457 & 0.2376 & 0.08 \\
\hline VasKK-b7 & 60 & 2.127 & 0.2123 & $0.0 \varepsilon$ \\
\hline
\end{tabular}

\section{Vasagård KK-b CI}

VasKK-b

VasKK-b2

VasKK-b3

VasKK-b

VasKK-b5

VasKK-b6

VasKK-b7

$\begin{array}{lrr}\text { mean } & \text { SD } \\ 45 & 0.6098 & 0.137 \\ 57 & 0.6772 & 0.2707 \\ 60 & 0.6375 & 0.1419 \\ 63 & 0.6016 & 0.0576 \\ 60 & 0.5744 & 0.086 \\ 60 & 0.562 & 0.0857 \\ 60 & 0.5475 & 0.0647 \\ & 0.60142857 & 0.12051429\end{array}$

\%

F-value d.f.

17.6614

d.f.

denomenator

$6^{d}$

p-value

0.0811

0.0792

0.0811

0.0811

\begin{tabular}{lrrr} 
source & \multicolumn{2}{c}{$\begin{array}{l}\text { sum of } \\
\text { of variation }\end{array}$} & \multicolumn{2}{c}{$\begin{array}{l}\text { degrees of } \\
\text { squares }\end{array}$} & \multicolumn{2}{l}{$\begin{array}{l}\text { mean } \\
\text { sum of }\end{array}$} \\
factor & 10.8229 & 6 & \multicolumn{2}{l}{$\begin{array}{l}\text { squares } \\
\text { error }\end{array}$} \\
total & 40.6489 & 398 & 0.1021 \\
F & 51.4718 & 404 &
\end{tabular}

$\mathrm{F}=1.8038 / 0.1021=176614$

$\begin{array}{lcc}\text { Cl } & \text { F-value } & \text { numerator } \\ 0.0401 & 17.7897 \\ 0.0356 & \\ 0.0347 & \\ 0.0339 & \\ 0.0347 & \\ 0.0347 & \\ 0.0347 & \end{array}$

denomenator

d.f. $\quad p$-value

$6 \quad 399 \quad 2.65 \mathrm{E}-18$

source

$\begin{array}{lll}\text { source } & \text { sum of degrees of sum of }\end{array}$ 
Vasagård KK-b Fe

VasKK-b2

VasKK-b3

VasKK-b4

VasKK-b5

VasKK-b6

VasKK-b7

VasKK-b1

VasKK-b2

VasKK-b3

VasKK-b4

VaskK-b5

VasKK-b6

VasKK-b7

$\begin{array}{ll}\text { mean } & \text { SD } \\ 45 & 0.3438 \\ 57 & 0.2777 \\ 60 & 0.4244 \\ 63 & 0.4576 \\ 62 & 0.4414 \\ 60 & 0.4141 \\ 60 & 0.3851\end{array}$

SD

0.1604
0.0972
0.1553
0.0938
0.1026
0.1113
0.114

$\begin{array}{lrrr}\text { factor } & 0.0391 & 6 & 0.0065 \\ \text { error } & 0.146 & 399 & 0.0004\end{array}$

$\begin{array}{lr}\text { total } & 0.1851 \\ \mathrm{~F}=0.0065 / 0.0004=17.9552\end{array}$

\begin{tabular}{|c|c|c|c|c|c|c|c|}
\hline & & SD & $95 \% \mathrm{Cl}$ & F-value & d.f. & $\begin{array}{l}\text { d.f. } \\
\text { d. }\end{array}$ & $p$-value \\
\hline 45 & 0.5249 & 0.2132 & 0.0367 & 25.5873 & 6 & 401 & $9.31 \mathrm{E}-26$ \\
\hline 57 & 0.4202 & 0.1105 & 0.0326 & & & & \\
\hline 60 & 0.5937 & 0.1849 & 0.0318 & & & & \\
\hline 63 & 0.66 & 0.0447 & 0.031 & & & & \\
\hline 63 & 0.6147 & 0.1407 & 0.031 & & & & \\
\hline 60 & 0.6339 & 0.0394 & 0.0318 & & & & \\
\hline \multirow[t]{5}{*}{60} & 0.6335 & 0.0496 & 0.0318 & & & & \\
\hline & & & & $\begin{array}{l}\text { source } \\
\text { of variation }\end{array}$ & $\begin{array}{l}\text { sum of } \\
\text { squares }\end{array}$ & $\begin{array}{l}\text { degrees of } \\
\text { freedom }\end{array}$ & $\begin{array}{l}\text { mean } \\
\text { sum of } \\
\text { squares }\end{array}$ \\
\hline & & & & factor & 2.4077 & 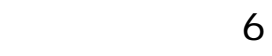 & 0.4013 \\
\hline & & & & error & 6.2889 & 401 & 0.0157 \\
\hline & & & & total & 8.6966 & 407 & \\
\hline
\end{tabular}

$\mathrm{F}=0.4013 / 0.0157=25.5873$

numerator denomenator

\begin{tabular}{|c|c|c|c|c|}
\hline & F-value & d.f. & d.f. & $p$-value \\
\hline 0.0352 & 15.7358 & 6 & 400 & $2.98 \mathrm{E}-16$ \\
\hline \multicolumn{5}{|l|}{0.0313} \\
\hline \multicolumn{5}{|l|}{0.0305} \\
\hline \multicolumn{5}{|l|}{0.0297} \\
\hline \multicolumn{5}{|l|}{0.03} \\
\hline \multirow{2}{*}{\multicolumn{5}{|c|}{$\begin{array}{l}0.0305 \\
0305\end{array}$}} \\
\hline \multirow{2}{*}{\multicolumn{5}{|c|}{0.0305}} \\
\hline & & & & mean \\
\hline & $\begin{array}{l}\text { source } \\
\text { of variation }\end{array}$ & $\begin{array}{l}\text { sum of } \\
\text { squares }\end{array}$ & $\begin{array}{l}\text { degrees of } \\
\text { freedom }\end{array}$ & $\begin{array}{l}\text { sum of } \\
\text { squares }\end{array}$ \\
\hline & factor & 1.3616 & 6 & 0.2269 \\
\hline & error & 5.7685 & 400 & 0.0144 \\
\hline & total & 7.13 & 406 & \\
\hline
\end{tabular}

Vasagård KK-b Ce/ Y

VasKK-b1
VasKK-b2
VasKK-b3
VasKK-b4

VaskK-b4

VasKK-b5

$\begin{array}{ll} & \text { mean } \\ 44 & 0.6278 \\ 57 & 0.8809 \\ 60 & 0.5473 \\ 63 & 0.3194 \\ 60 & 0.3823\end{array}$

numerator

denomenator

\begin{tabular}{lrccccr}
\multicolumn{2}{c}{$95 \% \mathrm{Cl}$} & \multicolumn{2}{c}{ F-value } & d.f. & d.f. & \multicolumn{2}{c}{ p-value } \\
0.6059 & 0.1075 & 17.9552 & 6 & 397 & $1.86 \mathrm{E}-18$ \\
0.3841 & 0.0945 & & & & & \\
0.5557 & 0.0921 & & & & \\
0.0737 & 0.0899 & & & & \\
0.2724 & 0.0921 & & &
\end{tabular}




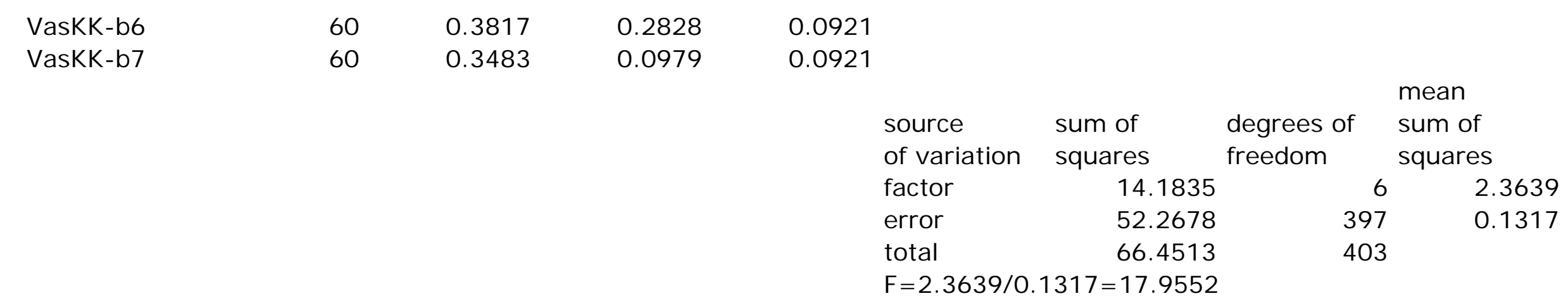

The Hagan MK-b null hypothesis is that all sub-samples cannot be distinguished on the basis of apatite chemistry, i.e. all sub-samples are similar. Analyzing the variance of all trace elements for the Hagan MK-b sub-samples yields a very high F-value with a very low probability.

The Hagan MK-b null hypothesis can be rejected.

\section{Hagan SUM $(\mathrm{Mg}+\mathrm{Cl}+\mathrm{Fe}+\mathrm{Mn}+\mathrm{Ce}+\mathrm{Y})$}

HagMK-b1

HagMK-b2

HagMK-b3

HagMK-b4

HagMK-b5

HagMK-b6

\begin{tabular}{rl}
\multicolumn{3}{c}{ mean } \\
89 & 0.7634 \\
102 & 0.6536 \\
105 & 0.7091 \\
93 & 0.7016 \\
111 & 0.6992 \\
83 & 0.6688
\end{tabular}

\section{$\mathrm{SD}$}

D $\quad 0.1339$

0.1339
0.0716

0.0933

0.0957

0.1142

0.0597

$95 \% \mathrm{Cl}$

F-value

$0.0205-13.8238$

0.0191

0.0188

0.02

\begin{tabular}{|c|c|c|c|}
\hline $\begin{array}{l}\text { source } \\
\text { of variation }\end{array}$ & $\begin{array}{l}\text { sum of } \\
\text { squares }\end{array}$ & $\begin{array}{l}\text { degrees of } \\
\text { freedom }\end{array}$ & $\begin{array}{l}\text { mean } \\
\text { sum of } \\
\text { squares }\end{array}$ \\
\hline factor & 0.6673 & 5 & 0.1335 \\
\hline error & 5.5702 & 577 & 0.0097 \\
\hline otal & 6.2375 & 582 & \\
\hline
\end{tabular}

$F=0.1335 / 0.0097=13.8238$

umerator denomenator

d.f. p-value

\section{Hagan Mg}

HagMK-b1

HagMK-b2

HagMK-b3

HagMK-b4

HagMK-b5

HagMK-b6 mean

$\begin{array}{rr}90 & 0.0336 \\ 102 & 0.0108\end{array}$

0.0108
0.0229

0.02

0.0363

0.0119
$0.022^{95 \%}$

0.022
0.0035

0.0058
0.03

0.0038
F-value

numerator denomenator

0.0032

0.0031

0.0033

0.003

0.0035

\begin{tabular}{|c|c|c|c|}
\hline $\begin{array}{l}\text { source } \\
\text { of variation }\end{array}$ & $\begin{array}{l}\text { sum of } \\
\text { squares }\end{array}$ & $\begin{array}{l}\text { degrees of } \\
\text { freedom }\end{array}$ & $\begin{array}{l}\text { mean } \\
\text { sum of } \\
\text { squares }\end{array}$ \\
\hline factor & 0.0559 & 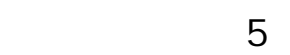 & 0.0112 \\
\hline error & 0.1523 & 578 & 0.0003 \\
\hline total & 0.2082 & 583 & \\
\hline
\end{tabular}




\section{Hagan $\mathrm{Cl}$}

HagMK-b1 HagMK-b2 HagMK-b3

HagMK-b4

HagMK-b5

HagMK-b6

90
102

90
102

105

93

111
83

0.3035 0.3035

0.2437

0.2357
0.212
0.2364

0.212
0.2364

0.1059
0.0141

9

$95 \% \mathrm{Cl}$

F-value

denomenato

0.0092

d.f. d.f. p-value

0.0091

$0.0127 \quad 0.0097$

$0.0275 \quad 0.0088$

$0.0146 \quad 0.0102$

\begin{tabular}{|c|c|c|c|}
\hline $\begin{array}{l}\text { source } \\
\text { of variation }\end{array}$ & $\begin{array}{l}\text { sum of } \\
\text { squares }\end{array}$ & $\begin{array}{l}\text { degrees of } \\
\text { freedom }\end{array}$ & $\begin{array}{l}\text { mean } \\
\text { sum of } \\
\text { squares }\end{array}$ \\
\hline factor & 0.4609 & 5 & 0.0922 \\
\hline error & 1.3002 & 578 & 0.0022 \\
\hline total & 1.7611 & 583 & \\
\hline
\end{tabular}

$\mathrm{F}=0.0922 / 0.0022=40.9791$

Hagan Fe

HagMK-b1

HagMK-b2

HagMK-b3

HagMK-b4

HagMK-b5

HagMK-b6

$\begin{array}{rl} & \text { mean } \\ 90 & 0.1311 \\ 102 & 0.0758 \\ 105 & 0.1167 \\ 93 & 0.1103 \\ 111 & 0.1283 \\ 83 & 0.0798\end{array}$

0.0395
0.0176
0.0163
0.017
0.0569
0.0193
$95 \% \mathrm{Cl}$

$$
\begin{array}{r}
\text { Cl } \\
0.0067 \\
0.0063 \\
0.0062 \\
0.0066 \\
0.0061 \\
0.007
\end{array}
$$

\section{Hagan Mn}

HagMK-b1

HagMK-b2

HagMK-b3

HagMK-b4

HagMK-b5

HagMK-b6

$\begin{array}{rr}{ }^{\text {mean }} & \text { SD } \\ 90 & 0.0514 \\ 102 & 0.0697 \\ 105 & 0.0462 \\ 93 & 0.0483 \\ 111 & 0.056 \\ 83 & 0.0703\end{array}$

SD

0.0135

0.0135
0.0099

$95 \% \mathrm{Cl}$

numerator

denomenator
51.7259
5
$578 \quad 2.40 \mathrm{E}-44$

d.f. d.f.

d.f. $\quad p$-value

$\begin{array}{llll}\text { source } \quad \text { sum of } \quad \text { degrees of } & \text { mean } \\ \text { sum }\end{array}$

\begin{tabular}{|c|c|c|c|}
\hline $\begin{array}{l}\text { source } \\
\text { of variation }\end{array}$ & $\begin{array}{l}\text { sum of } \\
\text { squares }\end{array}$ & $\begin{array}{l}\text { degrees of } \\
\text { freedom }\end{array}$ & $\begin{array}{l}\text { mean } \\
\text { sum of } \\
\text { squares }\end{array}$ \\
\hline factor & 1.3616 & 6 & 0.2269 \\
\hline error & 5.7685 & 400 & 0.0144 \\
\hline total & 7.13 & 406 & \\
\hline
\end{tabular}

of variation squares freedom

$\begin{array}{lrrr}\text { factor } & 0.2733 & 5 & 0.0547 \\ \text { error } & 0.6109 & 578 & 0.0011 \\ \text { total } & 0.8842 & 583 & \\ \mathrm{~F}=0.0547 / 0.0011=51.7259 & & \end{array}$

$\mathrm{F}=0.0547 / 0.0011=51.7259$

$\begin{array}{lll}0.056 & 0.014 & 0.0021\end{array}$ 
Hagan $\mathrm{Ce} / \mathrm{Y}$

HagMK-b1

HagMK-b1

HagMK-b2

HagMK-b3

HagMK-b4

HagMK-b5

HagMK-b6

$\begin{array}{rr}\text { mean } & \text { SD } \\ 89 & 2.121 \\ 102 & 0.9487 \\ 105 & 1.6666 \\ 93 & 1.575 \\ 111 & 1.2066 \\ 83 & 1.0002\end{array}$

SD $95 \% \mathrm{Cl}$

$\begin{array}{lll}1.4238 & 0.1322 & \text { F-value } \\ 0.2905 & 0.1235 & \\ 0.3972 & 0.1217 & \\ 0.2814 & 0.1293 \\ 0.3233 & 0.1184\end{array}$

$0.1322-46.9716$

0.3573

0.1369 numerator denomenator

d.f. $\quad 5^{\text {d.f. }} \quad 5_{77} \stackrel{\text { p-value }}{9.27 \mathrm{E}-41}$

\begin{tabular}{|c|c|c|c|}
\hline $\begin{array}{l}\text { source } \\
\text { of variation }\end{array}$ & $\begin{array}{l}\text { sum of } \\
\text { squares }\end{array}$ & $\begin{array}{l}\text { degrees of } \\
\text { freedom }\end{array}$ & $\begin{array}{l}\text { mean } \\
\text { sum of } \\
\text { squares }\end{array}$ \\
\hline factor & 94.6656 & 5 & 18.9331 \\
\hline error & 232.5747 & 577 & 0.4031 \\
\hline & 327.2403 & 582 & \\
\hline
\end{tabular}

The KK-b type locality null hypothesis is that the Mossen sub-samples are indistinguishable from the Vasagård sub-sample \#7.

On the basis of mean comparison of $\mathrm{Mg}, \mathrm{Cl}, \mathrm{Ce}$, and $\mathrm{Y}$ analyses the null hypothesis cannot be rejected.

On the basis of mean comparison of $\mathrm{Fe}$ and $\mathrm{Mn}$ analyses the null hypothesis can be rejected.

The difference in variance may be product insufficient analyses.

For example, the Mn and Fe differences between Mossen KK-b 20-26 and Vasagård KK-b \#4 are not sufficient to reject the null hypothesis.

The interpretation is that the KK-b type locaility displays sufficient similarities with the Vasagård KK-b such that the null hypothesis cannot be entirely rejected.

However, this must be interpreted with caution as the entire vertical profile of the Mossen KK-b cannot be sampled.

\section{Vasagård KK-b and Mossen KK-b (Mg)}

VaskK-b\#7

Mossen 20-26

Mossen $14-2 C$

$$
\text { mean }
$$

0.103

0.1005

\section{SD}

D

0.0209
0.0243

0.0201

$95 \% \mathrm{Cl}$

$0.0055^{\text {F-value }} 0.1706^{\text {d.f. }} \quad 2^{\text {d.f. }} \quad 154 \begin{aligned} & \text { p-value } \\ & 0.0064^{2}\end{aligned}$

numerator

$$
\text { d.f. }
$$

denomenator

\begin{tabular}{|c|c|c|c|}
\hline $\begin{array}{l}\text { source } \\
\text { of variation }\end{array}$ & $\begin{array}{l}\text { sum of } \\
\text { squares }\end{array}$ & $\begin{array}{l}\text { degrees of } \\
\text { freedom }\end{array}$ & $\begin{array}{l}\text { mean } \\
\text { sum of } \\
\text { squares }\end{array}$ \\
\hline factor & 0.0002 & 2 & 0.0001 \\
\hline & 0.0715 & 154 & 0.0005 \\
\hline & 0.0717 & 156 & \\
\hline
\end{tabular}

0.0057

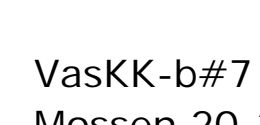

Vasagård KK-b and Mossen KK-b (Cl) $\mathrm{n}$ mean Mossen 20-26 60

Mossen 14-2C

\section{S}

$F=0.0001 / 0.0005=0.1706$

0.0427

F-value
0.9838

numerator denomenator

f.

d.f.

p-value

0.0181

source sum of degrees of sum of 


\begin{tabular}{lcrr} 
of variation & squares & freedom & \multicolumn{2}{c}{ squares } \\
factor & 0.0068 & 2 & 0.0034 \\
error & 0.5284 & 154 & 0.0034 \\
total & 0.5351 & 156 &
\end{tabular}

$\mathrm{F}=0.0034 / 0.0034=0.9838$

\section{Vasagård KK-b and Mossen KK-b ( $\mathrm{Ce} / \mathrm{Y})$}

VasKK-b\#7

Mossen 20-2e

Mossen 14-2C

0.3483

0.3252

0.3254
0.0979
0.067

0.0525
$95 \% \mathrm{Cl}$

numerato

-value denomenator

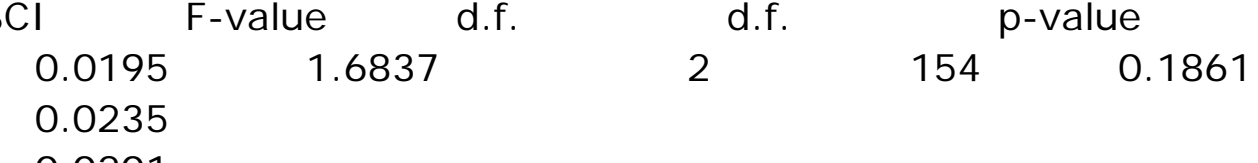

0.0201

source sum of degrees of sum of of variation squares freedom squares $\begin{array}{llll}\text { factor } & 0.0196 & 2 & 0.0098\end{array}$

$\begin{array}{llll}\text { error } & 0.8966 & 154 & 0.0058\end{array}$

total $\quad 0.9162$

$\mathrm{F}=0.0098 / 0.0058=1.6837$

Vasagård KK-b and Mossen KK-b ( Mn/ Fe)

VaskK-b\#7

$n$

mean SD

Mossen 20-26

60

60

0.5999

0.1467

$95 \% \mathrm{C}$

numerator denomenator

Mossen 14-2C

$56 \quad 0.4824$
0.0426

0.0426
0.0515

0.0515
0.0441

\begin{tabular}{|c|c|c|c|}
\hline $\begin{array}{l}\text { source } \\
\text { of variation }\end{array}$ & $\begin{array}{l}\text { sum of } \\
\text { squares }\end{array}$ & $\begin{array}{l}\text { degrees of } \\
\text { freedom }\end{array}$ & $\begin{array}{l}\text { mean } \\
\text { sum of } \\
\text { squares }\end{array}$ \\
\hline factor & 1.2426 & 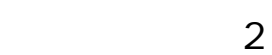 & 0.6213 \\
\hline error & 4.2975 & 154 & 0.0279 \\
\hline total & 5.5401 & 156 & \\
\hline$=0.6213 / 0$ & $79=22.2636$ & & \\
\hline
\end{tabular}

\section{Vasagård KK-b and Mossen KK-b ( Mn/ Fe)}

\section{VasKK-b\#4}

Mossen 20-26 mean SD

60.6891

0.71

$$
95 \%
$$
0.1228
0.1288

$95 \% \mathrm{Cl}$
0.0313
0.0388

numerator

F-value numerator denomenator

$0.0313 \quad 0.69333^{\text {d.f. }} \quad 1^{\text {d.f. }} 102^{\text {p-value }} 0.407$

$$
\text { source }
$$$$
\text { of variation squares freedom of sum of }
$$$$
\begin{array}{lrrr}
\text { of variation } & \text { squares } & \text { freedom } & \multicolumn{2}{c}{\text { squares }} \\
\text { factor } & 0.0109 & 1 & 0.0109 \\
\text { error } & 1.598 & 102 & 0.0157
\end{array}
$$$$
\begin{array}{lll}
\text { total } & 1.6088 & 103
\end{array}
$$ 
ortager than 17.8. 